\title{
QUALITY OF ANTIRETROVIRAL THERAPY IN PUBLIC HEALTH FACILITIES IN NIGERIA AND THE PERCEPTIONS OF THE END USERS
}

by

Robert Joseph Chiegil

submitted in accordance with the requirements

for the degree of

DOCTOR OF LITERATURE AND PHILOSOPHY

in the subject

HEALTH STUDIES

at the

UNIVERSITY OF SOUTH AFRICA

SUPERVISOR: Prof LI Zungu

CO-SUPERVISOR: Prof K Jooste

February 2012 
Student number: 3675-728-4

\section{DECLARATION}

I declare that QUALITY OF ANTIRETROVIRAL THERAPY IN PUBLIC HEALTH FACILITIES IN NIGERIA AND THE PERCEPTIONS OF END USERS is my own work and that all the sources that I have used or quoted have been indicated and acknowledged by means of complete references and that this work has not been submitted before for any other degree at any other institution.

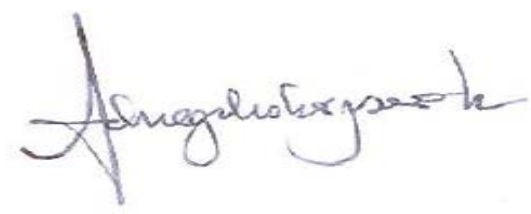

SIGNATURE

27 February 2012

(ROBERT JOSEPH CHIEGIL) 


\title{
QUALITY OF ANTIRETROVIRAL THERAPY IN PUBLIC HEALTH FACILITIES IN NIGERIA AND THE PERCEPTIONS OF THE END USERS
}

\author{
STUDENT NUMBER: \\ 3675-728-4 \\ STUDENT: \\ DEGREE: \\ Robert Joseph Chiegil \\ DEPARTMENT: \\ SUPERVISOR: \\ D Litt et Phil \\ Health Studies \\ Prof L Zungu \\ CO-SUPERVISOR: \\ Prof K Jooste
}

\section{ABSTRACT}

The health care industry in Nigeria is increasingly grappling with challenges of meeting end users' requirements and expectations for quality antiretroviral therapy (ART) service provision. This study sought to explore and describe the quality of antiretroviral therapy in public health facilities in Nigeria and the perceptions of the end users. A descriptive qualitative research design was used in the study in order to generate ideas from end users for improving quality of ART service provision, and prompt additional research activities. Unstructured focus group discussions were conducted with end users $(n=64)$ in 6 locations across the 6 geopolitical zones of Nigeria. Data was analysed using the framework approach because it reflects the original accounts and observations of the end users and the Weft QDA version 1.0.1 software to validate the results. Findings revealed that end users were satisfied with uninterrupted antiretroviral drug supplies, courtesy treatment, volunteerism of support group members and quality counselling services. End users expect public health facilities to accept diagnostic results from collaborating facilities, implement continuous quality improvement (CQI), maintain clean and adequate health infrastructure, reduce end user waiting time, reduce stigma, comprehensively assess end users during each clinic visit and ensure uninterrupted ART services. They also expect effective collaboration between healthcare providers and support group members, to enhance the quality of life of people living with HIV (PLHIV). End users identified the following as quality gaps in ART service provision: weak health facility leadership, non-attractive ART service infrastructure, frequently interrupted laboratory services, demotivated and inadequate health care workers, long waiting time, interrupted medicine supplies and inadequate procedure for complaints management. In conclusion, the following recommendations were proffered: deploy and 
train additional health care workers, integrate ART into regular health services, improve supply chain management of health commodities, and reduce end user overload in clinics. Finally, a best practice guideline for the provision of end user focused ART service provision was developed.

\section{KEY TERMS}

Perception, end users, quality, antiretroviral therapy, Nigeria, public health facility. 


\section{ACKNOWLEDGEMENTS}

I would like to thank the following persons and institutions for their valuable contributions to the undertaking and completion of this thesis:

- A special thank you to my supervisor, Prof $L$ Zungu, for her tireless guidance, support and encouragement

- $\quad$ My joint supervisor, Prof K Jooste, for her support and guidance

- $\quad$ My wife, Neilly R Chiegil, for her unconditional love, support and encouragement

- $\quad$ My three children, Changhenfemi, Changhieco and Changwami, for their support and understanding

- $\quad$ The University of South Africa (UNISA) Institutional Review Board and National Health Research Ethics Committee of Nigeria (NHREC), for granting permission and the people living with HIV and AIDS (PLHIV) support group leaders in the six locations that participated in the study, for their cooperation during the study

- $\quad$ The end users who took time off their busy schedules to participate in this study 


\section{Dedication}

This work is dedicated to my children, changhenfemi, changhieco and changwami 


\section{CHAPTER 1}

\section{ORIENTATION TO THE STUDY}

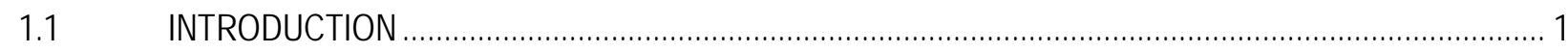

1.2 BACKGROUND INFORMATION RELATING TO THE RESEARCH PROBLEM .................................... 2

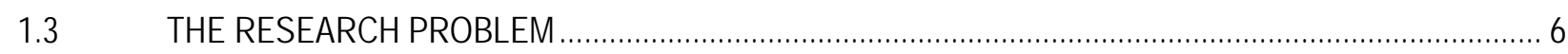

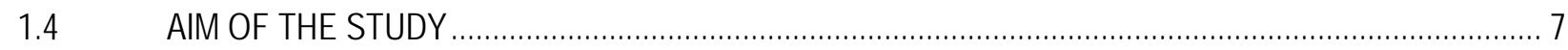

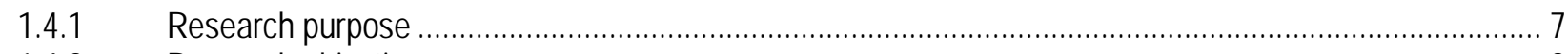

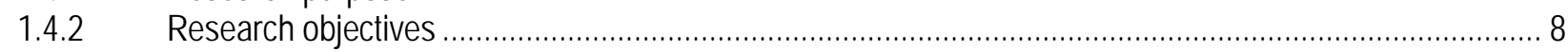

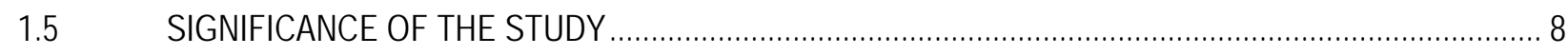

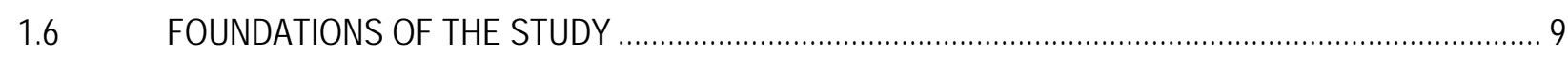

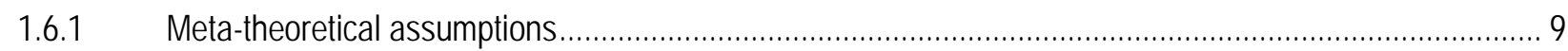

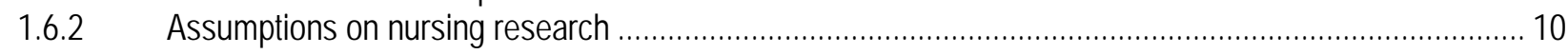

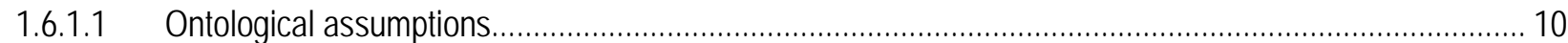

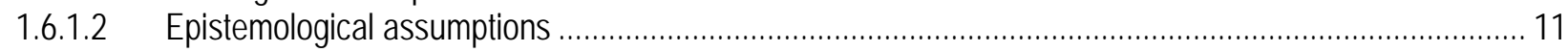

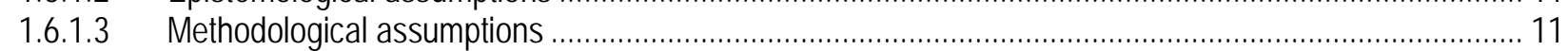

1.6.3 Theoretical framework for providing quality antiretroviral therapy and other definitions .......................... 15

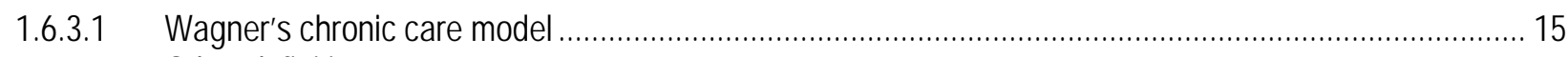

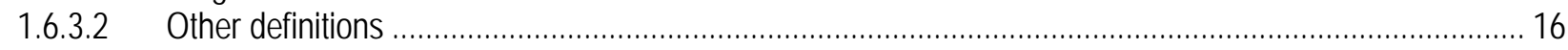

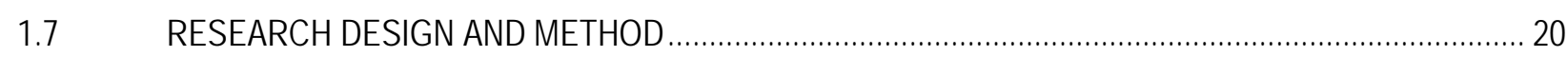

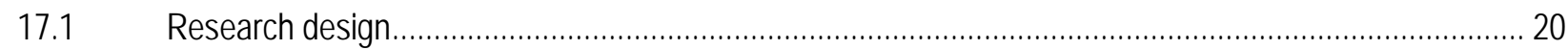

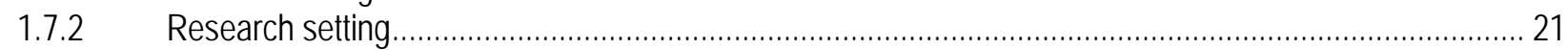

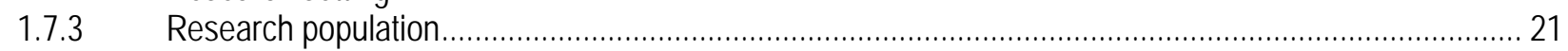

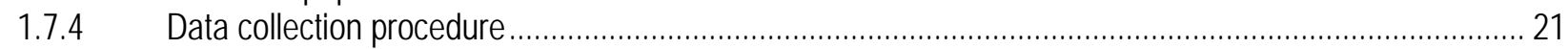

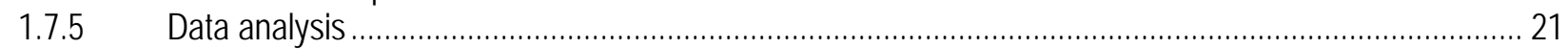

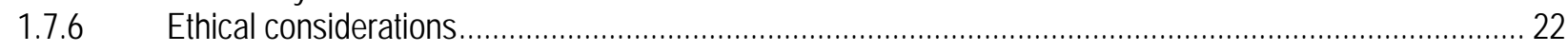

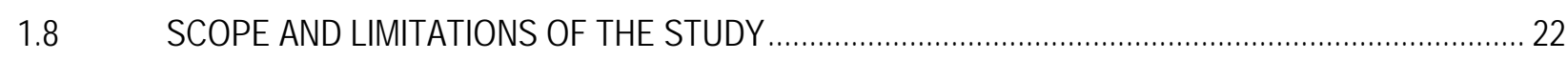

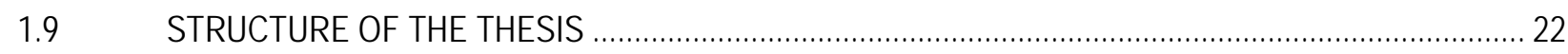

1.10 CONCLUSION 


\section{CHAPTER 2}

\section{LITERATURE REVIEW}

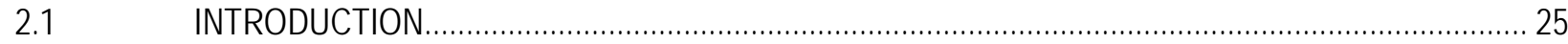

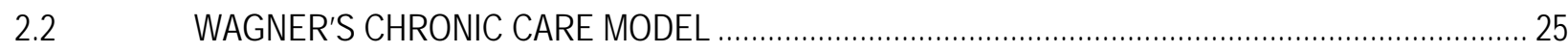

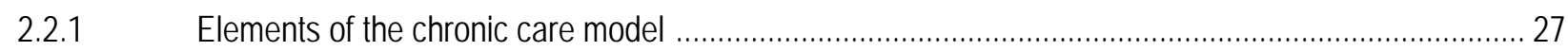

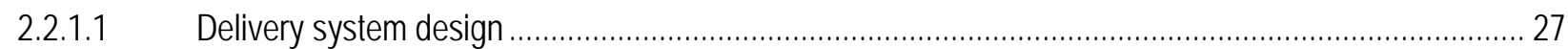

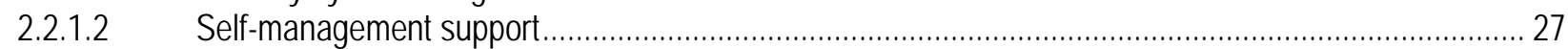

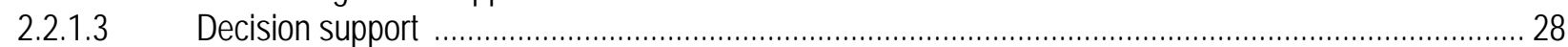

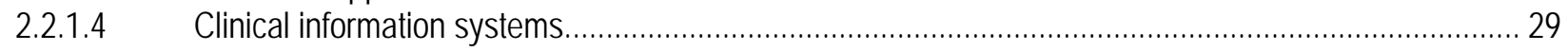

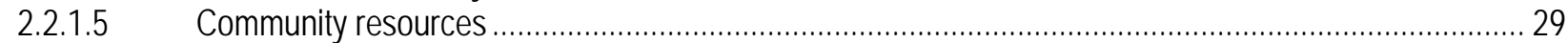

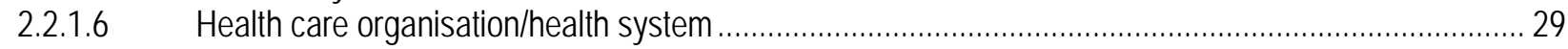

2.2.2 Justification for using the chronic care model in this study ........................................................... 29

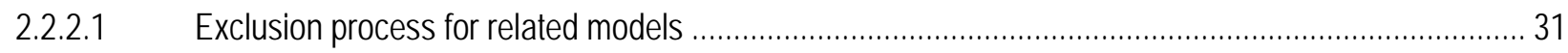

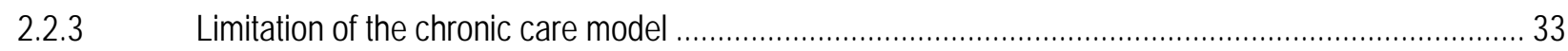

2.3 PERCEPTION OF END USERS CONCERNING QUALITY OF ANTIRETROVIRAL THERAPY .......... 33

2.3.1 Perception of end users concerning quality of antiretroviral therapy.............................................. 34

2.3.2 Perception of end users concerning quality of antiretroviral counselling services................................40 40

2.3.3 Perception of end users concerning quality of antiretroviral therapy pharmacy services in public

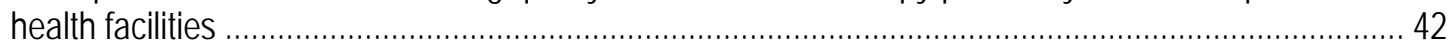

2.3.4 Perception of end users concerning quality of antiretroviral therapy laboratory services in public

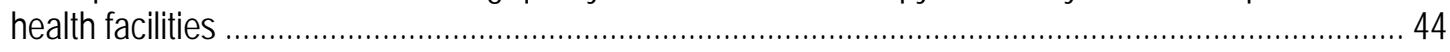

2.3.5 Perception of end users concerning antiretroviral pharmaceuticals based on material characteristics.. 45

2.3.6 Perception of end users concerning their interpersonal relationships with health care workers ............ 46

2.3.7 Perception of end users concerning respect for their choices/preferences for antiretroviral

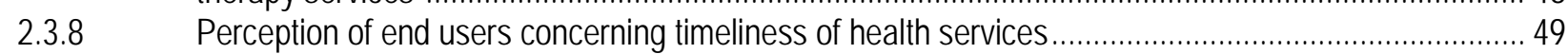

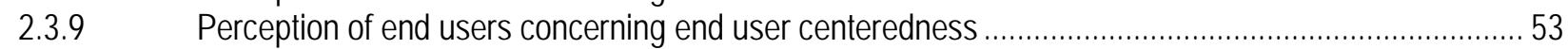

2.3.10 Perception of end users concerning end user safety .....................................................................5 57

2.3.11 Perception of end users concerning quality of communication and information shared with them .........59

2.3.12 Perception of end users concerning their rights to decision-making regarding their care .....................61

2.3.13 End users' perception concerning affective and cost-efficient ART service provision........................... 67

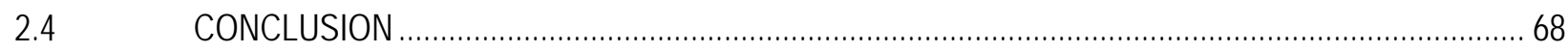

\section{CHAPTER 3}

\section{RESEARCH DESIGN AND METHOD}

$3.1 \quad$ INTRODUCTION. 


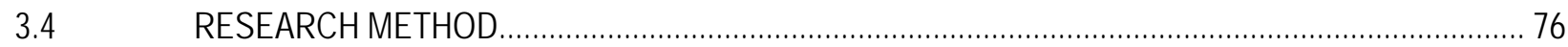

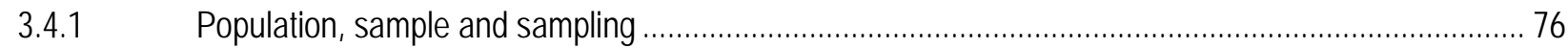

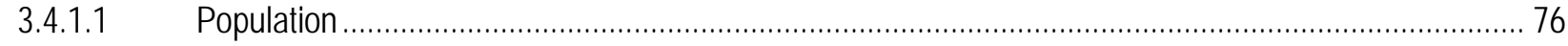

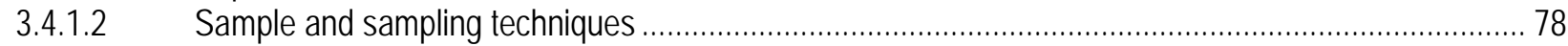

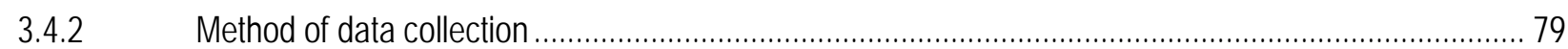

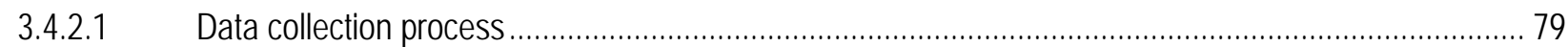

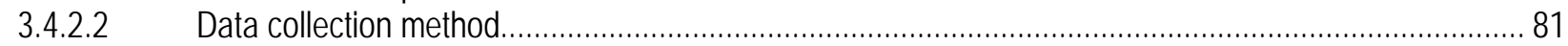

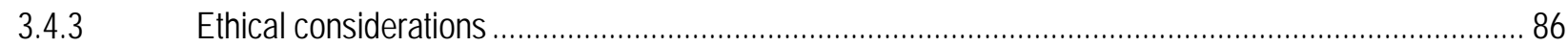

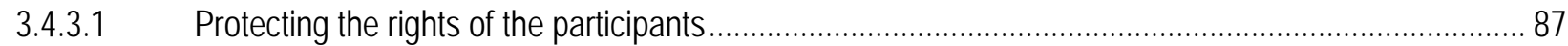

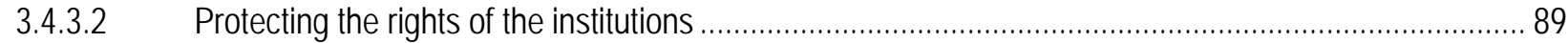

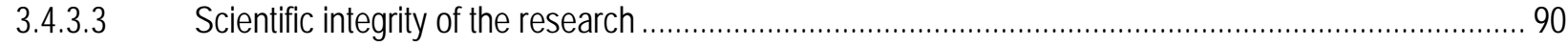

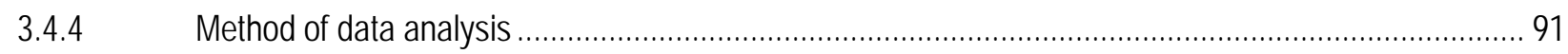

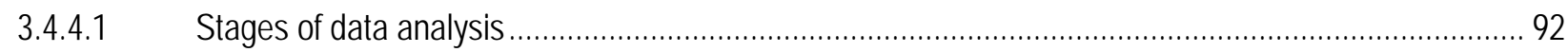

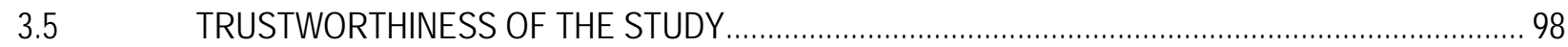

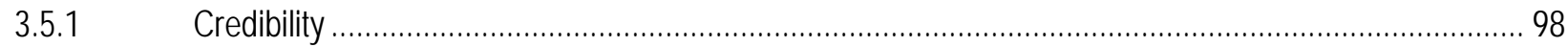

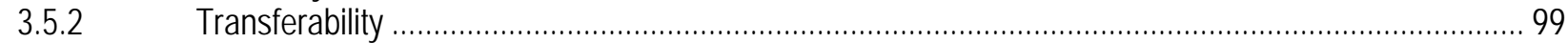

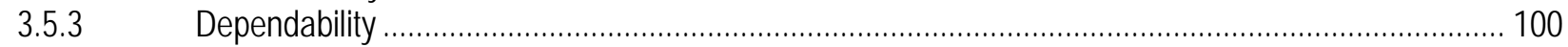

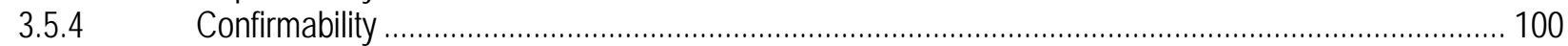

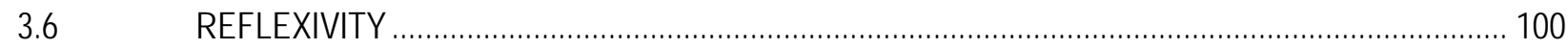

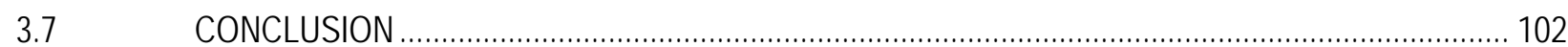

\section{CHAPTER 4}

\section{DATA ANALYSIS, PRESENTATION AND INTERPRETATION OF FINDINGS}

\begin{tabular}{|c|c|}
\hline 4.1 & INTRODUCTION \\
\hline 4.2 & OVERVIEW OF DATA ANALYSIS \\
\hline
\end{tabular}




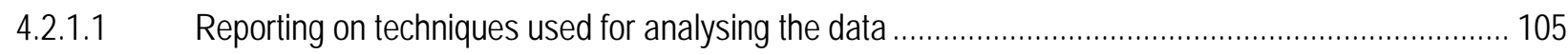

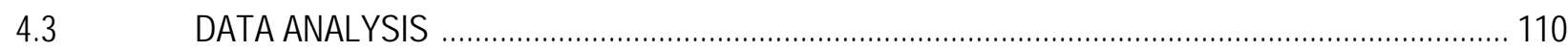

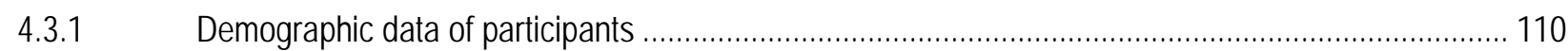

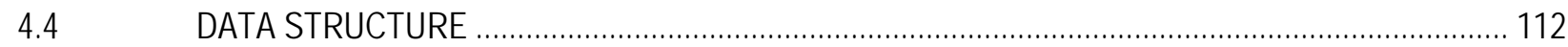

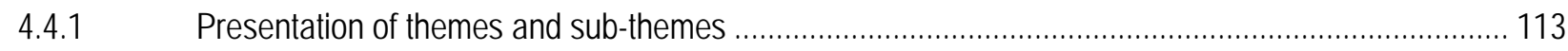

4.4.1.1 Theme 1: ART services that met participants' requirements......................................................... 113

4.4.1.2 Theme 2: Participants' perceived successes in ART service provision............................................. 129

4.4.1.3 Theme 3: ART services that disappointed participants from meeting their requirements

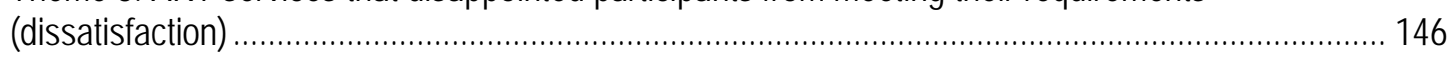

4.4.1.4 Theme 4: Participants' perception regarding their requirement for improving quality of ART

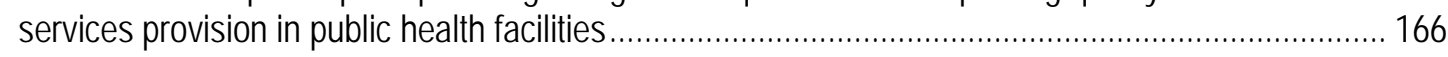

CONCLUSION

\section{CHAPTER 5}

\section{DISCUSSION OF FINDINGS}

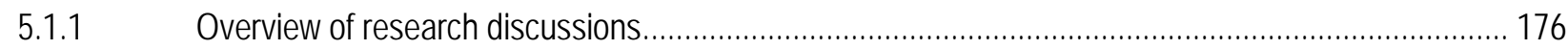

5.2 ART SERVICES THAT MET END USERS' REQUIREMENT ….................................................. 177

5.2.1 Uninterrupted supply of antiretroviral (ARV) drugs improve quality of life .......................................... 178

5.2.2 Courtesy service of doctors, nurses and other health workers serve as source of encouragement

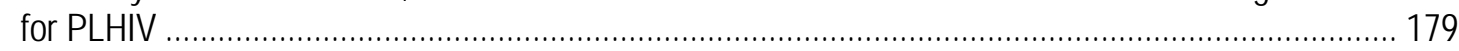

5.2.3 Empathising with the end user while counselling improves quality of life of the end user.................... 180

5.2.4 ART knowledge explosion improves cohesion among PLHIV and raises demand for quality of care.. 181

5.2.5 Enthusiam of support group volunteers to improve the quality of life of members .............................. 183

5.2.6 Support group volunteers promote infection prevention and control through triage of vulnerable members in the clinic ............................................................................................................ 184

5.2.7 Volunteer pharmacists improve quality of pharmacy services in ART clinics..................................... 186

5.2.8 Strengthening linkages between support groups and community based organisations provide opportunities for wrap around services and improves the quality of life of PLHIV ................... 188

5.2.9 Leadership of support groups implements innovative approaches to improve dialogue with health authorities to improve quality of life of people on ART …….......................................................... 189

5.3 THEME 2: PARTICIPANTS' PERCEIVED SUCCESSES IN ANTIRETROVIRAL THERAPY

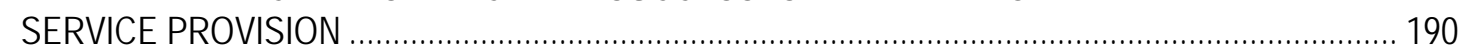

5.3.1 Health facilities are spacious, clean and attractive, with adequate infrastructure to meet environmental health standards and the aesthetic needs of end users 
5.3.2 Lifesaving ART programme is fundamental to the survival of PLHIV, thus, will not experience interruptions

5.3.3 Health facilities ensure that any end user who turns up to the health facility benefits from full assessment of his current health status; and is counselled on emerging clinical manifestations that needs medical attention

5.3.4 Health facilities recognise the essence of PLHIV as volunteers, and design capacity building and motivational programmes to meet both the task requirement and volunteers' expectations

5.3.5 Health facilities providing ART services have developed linkages with relevant facilities, to obtain specialised diagnostic services, and accept results arising from such facilities.

5.3.6 Health facilities has standardised processes that ensures end users spend the least amount of time seeking health care in the facility ..... Government, communities and individuals have ensured a stigma free environment for PLHIV ......... 204 Health facilities recognise that PLHIV loves interacting with their peers, and creates opportunities for such interaction as part of the continuum of care process ................................................................ 206

5.3.9 Health facilities continuously improve quality and processes to meet the changing requirement and expectations of end users .....

Perceived increasing incidences of failure to treat end users with courtesy, related to inadequate health care workers and staff burnout of the available few

Weak responsiveness to the needs of end users by the health facility leadership, policy and

5.4.6 Perceived increasing trends of inadequacy in ensuring end users emerge from the health facility healthy

5.4.8 Sub-optimal standardisation of health care processes and procedures, with resultant accumulation of bottlenecks along the workflow......

5.5 PARTICIPANTS' SUGGESTIONS FOR IMPROVING END USERS' REQUIREMENT FOR QUALITY ANTIRETROVIRAL THERAPY SERVICE PROVISION

5.5.1 Deploy, train and re-train additional health care workers to meet increasing number and dynamic expectations of end users in ART sites. Integrate HIVIAIDS into regular health services; end users encouraged to attend regular clinics ....... 229 Improve supply chain management of laboratory reagents and drugs for opportunistic infections, to ensure availability and use......

Reduce end user load in the clinic by reducing frequency of health facility visits by stable end users, through dispensing ARVs that will last 3 months per visit...... 


\section{CHAPTER 6}

\section{CONCLUSIONS, LIMITATIONS AND RECOMMENDATIONS}

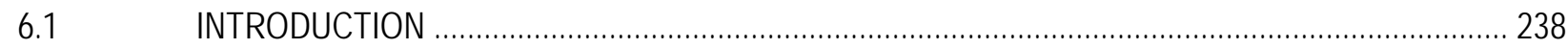

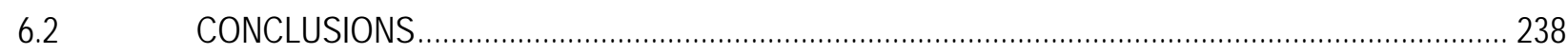

6.2.1 What are the perceptions of end users concerning the quality of ART in Nigerian public health

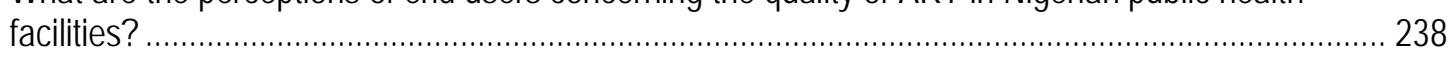

LIMITATIONS OF THE STUDY

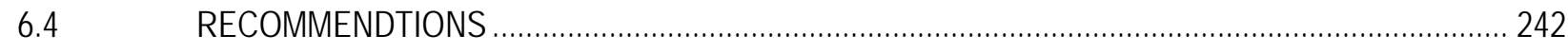

6.4.1 Recommendations for improving quality of ART care in public health facilities ............................... 242

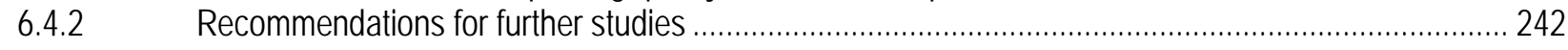

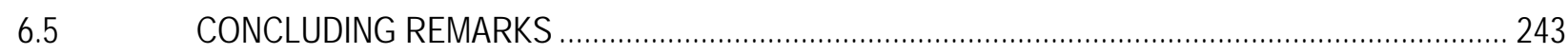

\section{CHAPTER 7}

\section{BEST PRACTICE GUIDELINES FOR PROVISION OF END USER FOCUSED ANTIRETROVIRAL THERAPY IN NIGERIAN PUBLIC HEALTH FACILITIES}

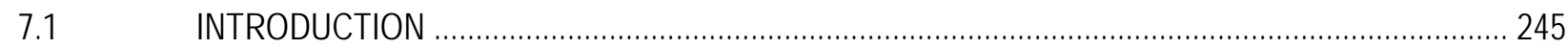

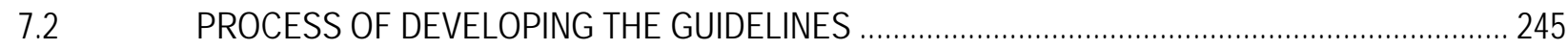

7.3 APPLICATION OF THE CONCEPTUAL FRAMEWORK TO THE DEVELOPMENT OF THE

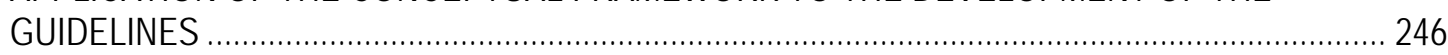

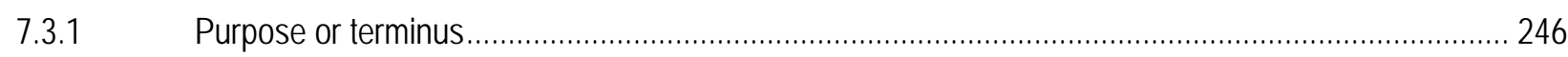

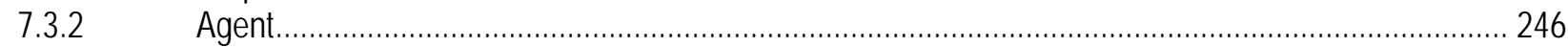

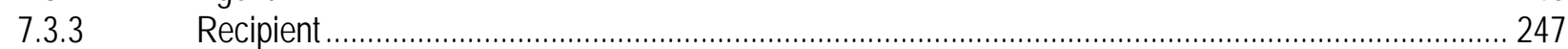

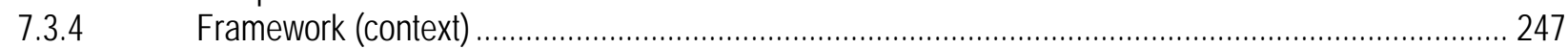

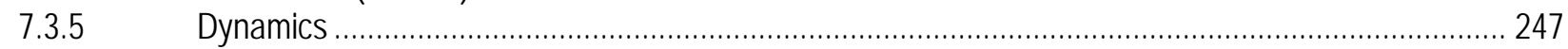

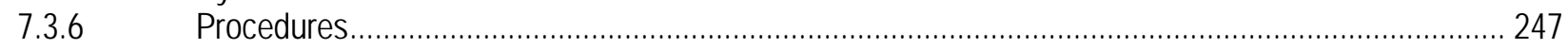

7.4 APPLICATION OF THE THEORETICAL FRAMEWORK TO THE DEVELOPMENT OF THE

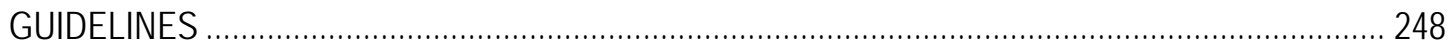

7.5 FORMULATION OF BEST PRACTICE GUIDELINES FOR PROVISION OF END USER FOCUSED ANTIRETROVIRAL THERAPY IN NIGERIAN PUBLIC HEALTH FACILITIES................... 258

7.5.1 THEME 1: Guidelines to address provision of ART services that meet end users' requirements ........ 258

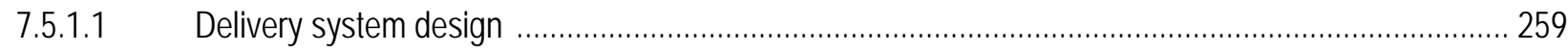


Self-management support

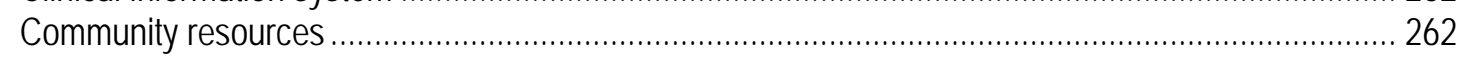

7.5.1.6

Health care organisation . 263

7.5.2 THEME 2: Guidelines to strengthen successes perceived by end users in ART 263

7.5.2.1

7.5 .2 .2

Delivery system design 263

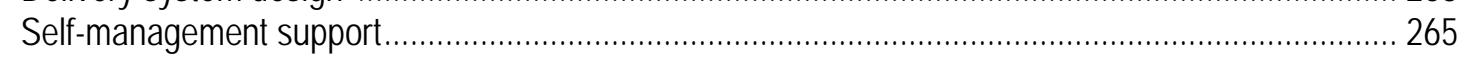

7.5 .2 .3

7.5 .2 .4

7.5 .2 .5

7.5 .2 .6

Decision support

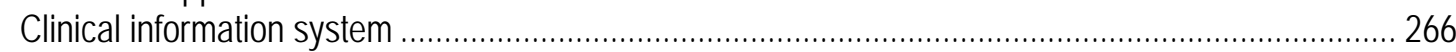

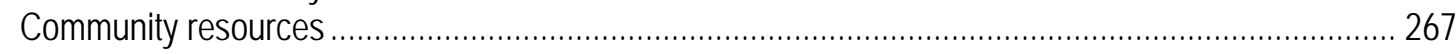

7.5 .3

Health care organisation

7.5.3 THEME 3: Guidelines to address ART services that failed to meet end users' requirement and expectations.

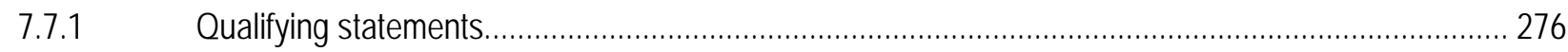

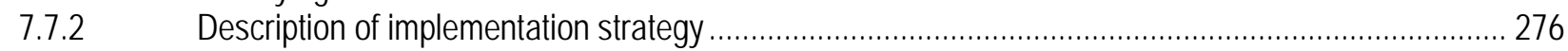

7.7.2.1 Implementation strategy using a self-care quality improvement model.............................................. 276

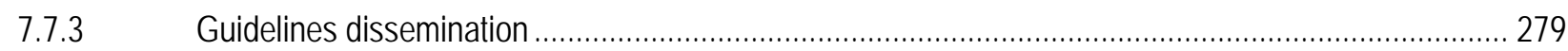

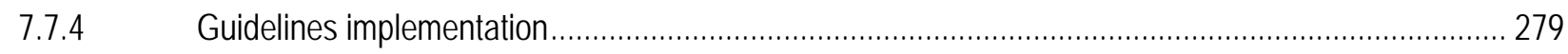

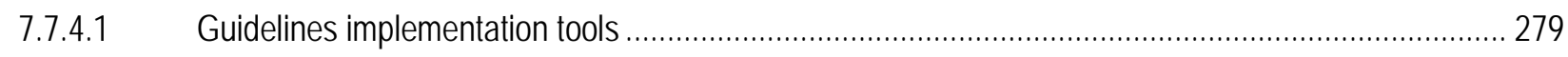

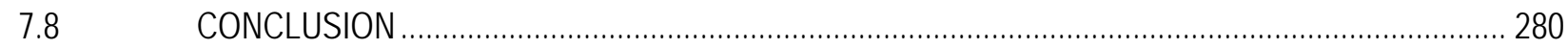

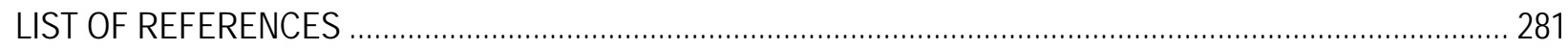




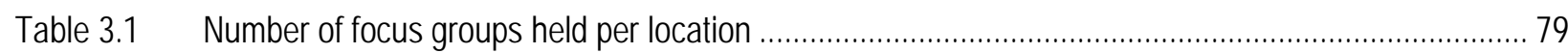

Table 3.2 Number of participants in focus groups held per location ................................................................. 79

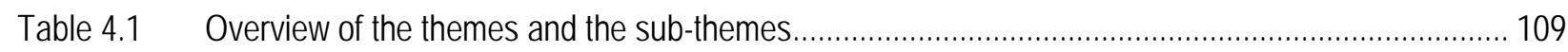

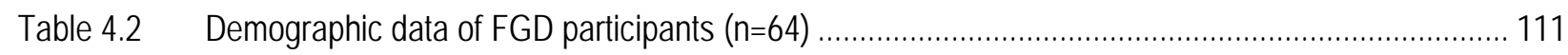

Table 4.3 Summary of findings regarding end users' perceptions concerning quality of ART service provision in Nigerian public health facilities

Table 7.1 Matrix showing best practice guidelines for provision of end user focused quality ART service provision in public health facilities. 
Figure 1.1 Conceptual framework illustrating end user focused quality of ART service provision 14

Figure 2.1 Wagner's Chronic Care Model. 28

Figure 3.1 Map of Nigeria showing the six geopolitical zones and the six public health facilities used as research sites for this study 73

Figure 3.2 Creating themes and sub-themes in Weft QDA version 1.0.1 software. 95

Figure 3.3 Screenshot showing themes (top left), sub-themes (lower left) and marked texts (lower central) during data analysis

Figure 3.4 Screenshot showing exported analysed data from Weft QDA version 1.0.1 software, in HTML format 97

Figure 4.1 Graphic hierarchical representation of themes and sub-themes as used in this study..... 108

Figure 4.2 Themes that emerged from the study 112

Figure 4.3 Hierarchical illustration of relationships in ART services that met end users' requirement 114

Figure 4.4 Hierarchical illustration of relationships between themes and 9 sub-themes in participants' perceived successes in ART services 130

Figure 4.5 Hierarchical relationships between theme 3 and its 8 sub-themes 147

Figure 4.6 Hierarchical relationships between participants' requirement for quality ART service provision 166

Figure 7.1 Self-care quality improvement process 


\section{List of abbreviations}

\begin{tabular}{ll} 
AIDS & Acquired Immune Deficiency Syndrome \\
ART & Antiretroviral Therapy \\
ARV & Antiretroviral \\
CPI & Continuous process improvement \\
CQI & Continuous quality improvement \\
DOT & Directly Observed Therapy \\
FHI & Family Health International \\
FGD & Focus Group Discussion \\
FMOH & Federal Ministry of Health \\
GHAIN & Global HIVIAIDS Initiative Nigeria \\
HAART & Highly Active ART \\
HCIP & Health Care Improvement Project (USAID) \\
HCW & Health care worker \\
HIV & Human Immunodeficiency Virus \\
IPD & In-patient department \\
IPPF & International Planned Parenthood Foundation \\
ITT & International Telephone \& Telegraph \\
JCAHO & Joint Commission on Accreditation of Health Care Organisations \\
M\&E & Monitoring and evaluation \\
NACA & National Agency for the Control of AIDS \\
NATG & National antiretroviral treatment group \\
NREC & National Research Ethics Committee \\
OPD & Out-patient department \\
PEPFAR & President's Emergency Plan for AIDS Relief \\
PLHIV & People living with HIV and AIDS \\
PMTCT & Prevention of Mother to Child Transmission of HIV \\
QA & Quality assurance \\
SSA & sub-Saharan Africa \\
STI & Sexually Transmitted Infection \\
TB & Tuberculosis \\
TQM & Total quality management \\
TQL & Total quality leadership \\
UK & United Kingdom \\
UN & United Nations \\
UNAIDS & Joint United Nations programme on HIVIAIDS \\
UNICEF & United Nations Children Emergency Fund \\
USA & United States of America \\
VCT & Voluntary counselling and testing \\
WHO & World Health Organization \\
\hline
\end{tabular}




\section{List of annexures}

Annexure A Certificate of participation in the training on research ethics

Annexure B $\quad$ Requests for permission to conduct the study

Annexure C Letter granting permission to conduct the study

Annexure D Ethical clearance certificate from Unisa

Annexure $\mathrm{E} \quad$ Consent letter for FGD participants

Annexure $F \quad$ Focus group discussion guide

Annexure $\mathrm{G} \quad$ Maps showing the 6 geopolitical zones of Nigeria

Annexure $\mathrm{H} \quad$ Cumulative number of end users receiving ART in the study sites 


\section{CHAPTER 1}

\section{ORIENTATION TO THE STUDY}

\section{$1.1 \quad$ INTRODUCTION}

Research examining end users' perceptions of the quality of antiretroviral therapy service provision (perceived service quality) in public health facilities is limited (Wouters, Heunis, Rensburg \& Meulemans 2008:2). Thus, for decades, end user satisfaction has been viewed as an indicator of quality care that is delivered in health care services (Clearly \& McNeil 1988:25; Gill \& White 2009:12). In the past decade in particular, Wouters et al (2008:2) and Liu and Wang (2007:266) reported that end user satisfaction has become an important performance measure and outcome of health care. This includes the ability to indicate differences in end user satisfaction with health services among heterogenous populations (Lima-Costa \& Filho 2008:247).

Antiretroviral therapy service provision requires high end user involvement in the consumption process, and the traditional health care view of technical quality is inadequate to manage this complexity (Gill \& White 2009:14). More importantly, Gill and White (2009:14) argue that effective health care relies on the co-contribution of and attention given to the end user to the service delivery process.

Porzsolt, Ackermann and Amelung (2007:2) applied economic theory to elucidate the measurement of the ability of an object to satisfy the needs of an end user (value) in antiretroviral therapy service provision. Porzsolt et al (2007:2) affirm that ten minutes of a health care worker's full attention and the perception of empathy are much more valuable to end users than twenty business minutes of the health care worker's time budget. In other words, a formal expression of the value of health care may be plausible for policy considerations of health care managers, but not for individual end users who have a serious problem (Porzsolt et al 2007:2). This elucidates the importance of end user satisfaction studies in antiretroviral therapy services. Studies have shown evidence that when end users participate in their care, they are more likely to be satisfied with the care. Compliance with medical advice and treatment regimens is in turn, directly related to the perceived quality of the service, antiretroviral therapy adherence and the 
subsequent resulting health outcome (Wouters et al 2008:5; Gill \& White 2009:14). Additionally, satisfied end users are likely to take an active role in their own care, continue using medical care services, and stay with a health care worker even when there are other choices (Udoh, Eze \& Okeji 2011:220). Udoh et al (2011:220) also suggest that health professionals and managers of health institutions may benefit from satisfaction studies that identify potential areas for service improvement, and health expenditure may be optimised through end user-guided planning and evaluation. End users' perception of quality of antiretroviral therapy in public health facilities in Nigeria is relatively unexplored.

Therefore, this study sought to explore and describe the perceptions of end users concerning the quality of antiretroviral therapy (ART) in Nigerian public health facilities. The study also sought to explore and interpret the expectations of end users concerning the quality of ART in Nigerian public health facilities to support the development of user focused best practice guidelines for ART. This chapter provides the background information related to the focus of the study, which highlights the core issues that stimulated the researcher's interest to investigate on this topic. Also the chapter highlights the research problem, aim and significance of the study and the foundations, research design and methods of the study. It also highlights the scope and limitations, and the overall structure of the study.

\subsection{BACKGROUND INFORMATION RELATING TO THE RESEARCH PROBLEM}

Gill and White (2009:14) report that historically, the management of health care quality has been the responsibility of the service provider, focusing mainly on the technical provider components. Except for some studies in clinical governance which sought to emphasise the importance of the end user perspective, there was comparatively little work investigating end user perceptions of health care quality (Gill \& White 2009:14). This notion of health care quality negates the health care quality system which guarantees the continuum of care, not merely for curative services, but for health promotion as well as prevention (Shaikh 2005:515). Shaikh (2005:515) laments that the notion of end user satisfaction studies emerged more strongly in recent times because of the rising costs of treatment, constrained resources in health services and evidence of variations in clinical practice. Gill and White (2009:10) on the other hand point out that the need for the measurement of end user satisfaction with antiretroviral services 
has been largely driven by the underlying politics of "new public management," mainly driven by health care managers to meet end user requirement and expectations. The concomitant rise in the health consumer movement, with end user satisfaction being one of the articulated goals of health care delivery, was another factor highlighted by Gill and White (2009:10). Measurement of end users' perception of quality of antiretroviral therapy service provision is in fact, respecting the consumer sovereignty, and a paradigm shift from the traditional measures of morbidity and mortality patterns and other methods such as physician peer reviews (Shaikh 2005:515).

An understanding of how end users evaluate their care may therefore, help to identify deficiencies and inform improvements. This understanding will help to render health care programmes in a more end user centred manner, and to increase their efficiency in a context of scarce resources (Wouters et al 2008:2). Chow, Mayer, Darzi and Athanasiou (2009:442) opine that by measuring end user satisfaction reliably, we can mould our health care systems around the end user, the person who matters most. With the advent of end user rights movement, the debate over the relationship between end user satisfaction as a valuation of the process of care versus the standard of technical care became widespread (Gill \& White 2009:10). For example, assessing end user satisfaction has been mandatory for French hospitals since 1998, to improve end user satisfaction in a consumerist sense, but not necessarily to improve care (Gill \& White 2009:10).

Gill and White (2009:12) argue that since there is no consensus on how best to conceptualise the relationship between end user satisfaction and their perceptions of quality of health care, focus should be on measuring technical and functional (how care is delivered) quality and not end user satisfaction. In the views of Coulter (2005:1199), end users care more about the quality of their everyday interactions with health professionals and not how technical health services are organised. Holding a separate view is Chow et al (2009:438) who state that understanding the concept of end user satisfaction is important on many levels. First, the measurement of end user satisfaction allows health care workers to change their practice to improve the quality of care to improve health outcomes, reduce cost and keep end users happy, in addition to many other beneficial effects. On an individual level, Chow et al (2009:438) report that improved satisfaction with care would result in greater cost savings and user compliance with care. At a national level, Chow et al (2009:438) note that attention to 
end user satisfaction and a drive to increase satisfaction with services will help to improve the public's perception of the health care system. The measurement of end user satisfaction can also affect wider interest of the population's health and health care policy. End user satisfaction studies could ultimately form a component of a world class commissioning process that aims to deliver better care to the population (Chow et al 2009:439).

Collins, Nicolson and Bowns (2000:81) lament that the end users' experiences can be an important lever for change, both highlighting where, and what sort of quality improvements are needed. Within the United Kingdom (UK) National Health Service (NHS), Collins et al (2000:82) report that the role of the end user has changed and user satisfaction has increased in importance. From satisfaction studies, we are able to understand more about end users' experience of health care, promote co-operation with that care and increase compliance with treatment (Collins et al 2000:82). They also help the health professionals to identify problems in health care and evaluate care. Collins et al (2000:82) maintained that user satisfaction studies also help to understand the end user-health care worker communication process and predict end users' health-related behaviour, utilisation of care and continuity with the same provider.

Wouters et al (2008:2) posit that satisfied end users are more likely to comply with antiretroviral therapy, which is in turn associated with better clinical outcomes. On this point, Chow et al (2009:438) elucidate that this outcome would lead to beneficial changes in the health of the population. Similarly, there may be less misuse of health care services. Together with a boost in morale and the provision of positive feedback to health care workers, efficiency and productivity of the health care system as a whole will improve (Chow et al 2009:439). In agreement with this assertion, Gill and White (2009:14) report that there could be significant reductions in the total cost of care when the end user's perception of the quality of services improves. The dynamics of poor service delivery often involves wasted effort, repetition and misuse of skilled employees (Gill \& White 2009:14).

HIVIAIDS represents one of the major worldwide health problems that have had a shattering impact on many countries in the past decade, especially those in subSaharan Africa (WHO 2004:2). As a result, public health facilities providing ART 
services in Nigeria are increasingly grappling with challenges of meeting end users' requirement for quality $A R T$ service provision.

WHO (2004:3) reports that the perception of the needs of an end user varies with the different perceptions and perspectives of the end user, health care provider and the social, political and economic environment. While the government of Nigeria responded to developing national policy document and guidelines that address the perceptions of the health care provider and the social, political and economic standpoints, little has yet been done to meet the perceptions of the end users. A national guideline for the provision of end user focused ART that will address quality ART service provision from the perspective of the end user is yet to be developed in Nigeria. As a result, social or cultural factors influence health care workers' negative perceptions regarding ART service provision to their end users. For example, Dao, Ferreira, Vallier, Roulin, Hirschel and Calmy (2010:185) reported that end users from sub-Saharan Africa (SSA) infected with HIV and living in Switzerland, disagreed with their physicians' evaluation of their health status. That is, while physicians deduced improved health status from laboratory results, these did not provide an adequate surrogate marker of good health for end users. End users experienced important social and economic difficulties with adverse consequences on their mental health. They requested social assistance, whereas physicians sought improved cultural competency. This corroborates the assertions by Creel, Sass and Yinger (2002:2) that health care quality is a multi-dimensional issue that may be defined and measured differently, according to stakeholders' priorities:

a) End users, whose perception of quality may be influenced by social and cultural concerns, place significant emphasis on the human aspects of care.

b) Providers usually stress the need for technical competency, as well as infrastructure and logistical support from their institution.

c) Programme managers may focus on support systems, such as logistics and record-keeping.

d) Policy makers and donors are concerned with cost, efficiency, and outcomes for health investment as a whole. 
This illustrates the importance of accessing end users' perspective of quality ART service, and not relying solely on health care workers' or other stakeholders' perception of what constitutes quality of ART service provision.

Creating an end user focused health facility is an attempt to removing barriers in delivery of quality ART care. Mehrabi, Nasiripour and Delgoshaei (2008:563) espouse that recently, in the business world and service rendering markets, emphasis is placed on the end user. This depicts that the quality of ART services should be specified on the basis of end users' needs and requirements rather than theoretical standards. Therefore, the most successful quality improvement plans have been those recognising and defining the quality from the viewpoint of the end users (Mehrabi et al 2007:563).

In fact, satisfied end users are considered as income sources for the health facilities and those health facilities that are unable to obtain the satisfaction of their service users will gradually vanish in competitive markets. Therefore, it is required that relationships between health facilities and end users be improved and all measures taken in the health facility be directed toward the recognition of end user needs (Mehrabi et al 2007:563). In health services organisations, the effectiveness of quality improvement model is evaluated with regard to the satisfaction of end users (Mehrabi et al 2007:563).

It was against this background that this study sought to determine the perceptions of end users concerning the quality of ART service provision in Nigerian public health facilities, in order to develop practical guidelines for implementation of best practice quality service provision in ART, which is end user focused.

\subsection{THE RESEARCH PROBLEM}

Consumerism has increasingly prompted end users attending ART services in Nigerian public health facilities to question the quality of ART service provided to them. Todays' end users of ART services are therefore sophisticated, demanding, and more educated than ever before. They have alternatives, a variety of hospitals, centres for holistic health and the like. Yet, the researcher observed that similar to what was reported by Dao et al (2010:185) in Switzerland, health care workers pay inadequate attention to meeting end user needs and expectations for quality ART service provision. If health services are to survive, and AIDS treatment outcomes are to be improved, end users 
must choose services on the basis of quality and responsiveness to their needs. The Nigeria national guidelines for ART service provision, are mainly provider focused, and have failed to address quality of ART service provision with a focus on the end users. A perspective of the end users is important. Thereby, ART services in Nigerian public health facilities are compromising what can be termed as quality ART service provision otherwise elucidated as safe, effective, timely, end user focused and efficient ART care. In other words, the Nigeria national ART guidelines lack the complement of end user focus which addresses perceptions on the fulfilment of needs and expectations (satisfaction) of the end users.

In order to solve the above problem of suboptimal quality ART service provision from the perspective of the end users, this study sought to answer this broad question:

"What are the perceptions of end users concerning the quality of ART service provision in Nigerian public health facilities?"

The researcher was furthermore unclear on what actions should be taken in user focused best practice guidelines for ART service provision in Nigerian public health facilities.

It is envisaged that the results of this study will inform the development of an end user focused best practice guideline that will promote quality of ART provision in Nigerian public health facilities.

\subsection{AIM OF THE STUDY}

\subsubsection{Research purpose}

The purpose of this study was to develop best practice guidelines that are user-focused to promote the quality of ART service provision in Nigerian public health facilities. 


\subsubsection{Research objectives}

In order to achieve the purpose of this study the following objectives were formulated:

- $\quad$ explore and describe the perceptions of end users concerning the quality of ART service provision in Nigerian public health facilities

- $\quad$ develop user focused best practice guidelines for ART service provision at Nigerian public health facilities

- $\quad$ validate the practice guidelines for ART service provision at Nigerian public health facilities

- $\quad$ present recommendations for implementation of the guidelines for ART service provision at Nigerian public health facilities

\subsection{SIGNIFICANCE OF THE STUDY}

The results of this study should add to the existing body of knowledge regarding development of best practice guidelines that will promote end user focused quality of ART service provision in Nigerian public health facilities, specifically, and in SSA in general. Policy makers should consider the results of this study to develop policies that will improve the quality of ART service provision, by assuming a paradigm shift from focus on health care worker to end user focused quality of ART service provision. Health care workers should utilise the results of this study to improve the overall quality of ART service provision in public health facilities in Nigeria. If the quality of ART service provision in public health facilities in Nigeria could be enhanced through end user focused care, ART retention and treatment outcomes should improve, while as a long term goal, HIVIAIDS mortality and morbidity rates would decline. Moreover the spread of potentially ARV-resistant strains of HIV throughout the communities could be contained.

The results of this study will contribute to an understanding of the perceptions of end users on ART service provision at Nigerian public health facilities. It will also improve the quality of health services in this regard. The best practice guidelines developed from the study could enable stakeholders to improve the ART service provision at Nigerian public health facilities. 


\subsection{FOUNDATIONS OF THE STUDY}

\subsubsection{Meta-theoretical assumptions}

Assumptions are statements that are accepted as true without verification (Polit \& Beck 2004:13). An assumption is a proposition or statement whose truth is either considered self-evident of what has been satisfactorily established by earlier research (Moleki 2008:24; Burns \& Grove 2003:157).

Moleki (2008:25) elucidates that the term "meta-theory" refers to critical reflection on the nature of scientific inquiry. Meta-theoretical reflection typically addresses issues such as the nature and the structure of scientific theories, the nature of scientific growth, the meaning of truth and, explanations (Babbie \& Mouton 2002:20). Meta-theoretical assumptions are interrelated sets of concepts, beliefs, commitments and propositions that constitute the study (Henning, Van Rensburg \& Smit 2004:15). They denote commitment to the truth of the theories and laws of a particular paradigm. Their origin is philosophical in nature, and therefore not meant to be tested. Creswell (2003:11) postulates that qualitative research focuses on the process occurring as well as the product. In this study the product will refer to best practice guidelines for the provision of quality ART services.

The meta-theoretical assumption in this study comprises person, environment, health and nursing (Walker \& Avant 2004). The study on end users and the quality of ART service provision in Nigerian public health facilities were intricately linked to nursing practice and related to the end user (person), public health facilities (environment), ART service provision (health) and quality (health services).Therefore, the following operational definitions support key meta-theoretical assumptions made in this study:

End user (person/end user) is an adult end user (client), male or female, who is aware of his/her rights to health care and attending antiretroviral therapy in a public health facility in Nigeria. Each person is viewed as a unique human being with own perceptions (internal environment). The person is in interaction with the external environment. 
Public health facility (external environment) is a clinical setting established and managed by the government of Nigeria, to meet the health care needs and expectations of end users attending ART. The environment comprises both the physical (antiretroviral therapy clinic and resources) and social (health care workers) dimensions. In this study "environment" refers to the factors/conditions (social or cultural) that influence the end user either directly or indirectly in his/her involvement in the antiretroviral therapy in public health facilities in Nigeria.

Quality antiretroviral therapy (health) is two fold. The quality of care aspect is the core of ART service provision. Quality refers to meeting the requirement and expectations (satisfaction) of end users at minimal cost. As a result of care, the health care worker moves away from his/her own view point and looks at things from the viewpoint of end users and makes them his/her priority. User focused best practice guidelines for ART service provision enables the health care worker to carry out quality ART service provision that meets the requirement and expectations of end users.

Antiretroviral therapy refers to the prescription of ARVs, laboratory monitoring, management of opportunistic infections, and the end user taking the ARVs at the correct times every single day of one's entire life, adhering to food prescriptions, and taking generally good care of own health. ART also involves end users attending follow-up clinic visits and health care workers maintaining available supplies of ARVs to end users.

\subsubsection{Assumptions on nursing research}

\subsubsection{Ontological assumptions}

Ontological assumptions are statements about human nature, society, nature of history and material phenomena, causality and intentionality (Mouton 1996:46). Bell (2002:209) refers to ontology as the study of the nature of existence and of coming to being. The ontological assumptions deal with the nature of reality as an object of inquiry (Moleki 2008:26). Against the backdrop that reality is dynamic; the following ontological assumptions were used in this study, to nurture reality in understanding the perceptions of end users concerning the quality of ART service provision in Nigerian public health facilities: 
- $\quad$ End users attending ART services in public health facilities understand the nature of treatment and its outcomes.

- $\quad$ End users attending ART services in public health facilities are sophisticated, demanding, more educated than ever before, and have alternatives to seek health care.

- $\quad$ End users attending ART services in public health facilities no longer idolise health care workers; they now demand higher quality of ART services.

\subsubsection{Epistemological assumptions}

Epistemological assumptions relate to assumptions about the nature of knowledge and science or the content of truth and related ideas (Mouton 1996:123). Epistemological assumptions are theoretical perspectives of interrelated sets of assumptions, concepts and propositions that constitute a view of the world (Henning et al 2004:15). In order to espouse the nature of knowledge and the content of truth in understanding the perceptions of end users concerning the quality of ART service provision in Nigerian public health facilities, the following epistemological assumptions were made:

- $\quad$ The diverse perceptions of end users assist in shaping the future of quality of ART service provision.

- Quality of ART service provision makes public health facilities efficient, effective and end user focused.

- $\quad$ End user focused ART service provision improves adherence on ART, retention on ART, and consequently, positive AIDS treatment outcomes.

These broad assumptions were stated to clarify the existence of the phenomenon under study, and not in any way, meant to describe, pre-empt or predict any theory.

\subsubsection{Methodological assumptions}

Methodological assumptions relate to the nature of research processes and the appropriate methods for investigating the phenomena of interest to the researcher (Mouton 1996:124). The descriptive and explorative qualitative research method was assumed to be the appropriate method for investigating the perceptions of end users 
concerning quality of ART service provision in Nigerian public health facilities. Qualitative research enables the researcher to investigate the perceptions of end users concerning their lived experiences, requirement and expectations of quality of ART service provision. A decision to use focus group discussions was driven by the need to experience albeit in an artificial setting, the experiences, expectations and perceptions of the participants.

\section{- $\quad$ Conceptual framework}

A conceptual framework will be used together with the Wagner's Chronic Care Model (CCM) to link the findings of the study to the body of knowledge and conceptualise this in practice through development of end user focused best practice guidelines for ART service provision (Moleki 2008:28; Burns \& Grove 2003:799). According to Moleki (2008:28), a conceptual framework provides a rationale or structure that guides the development of the study. It forms an understanding on which the study is based (Moleki 2008:28). It is made up of propositions, sets of concepts and statements integrated into a meaningful configuration (Fawset 2005:34).

Moleki (2008:28) elucidated a survey list based on the survey list of Dickhoff, James and Wiedenbach (1986) that was used as a conceptual framework for this study to describe the process for developing an end user focused best practice guidelines for quality ART service provision. The concepts used, purpose or terminus, the agent, recipient, framework or context and procedures were linked to person/end user, the environment/public health facility, health/ART service provision and health services/quality which are concepts (Oerman 2001:32) that are used to explain the assumptions and the perceptions of end users concerning the quality of ART service provision in Nigerian public health facilities.

Moleki (2008:28) described the survey list as follows:

The 'purpose' or 'terminus; relates to the type of activities, their boundaries and goals that a person engages in. In this study the purpose was to explore and describe the perceptions of end users (person) concerning the quality of ART service provision, in order to develop a user focused best practice guidelines for ART service provision. 
The 'agent' according to the survey list is someone who has the knowledge and ability to perform identified activities or provides a solution to a problem. In this study the agent was the health care worker or professional qualified to provide quality ART services in Nigeria. These health care workers include nurses, pharmacists, doctors, laboratory scientists and other paramedical staff.

The 'recipient' is the beneficiary of the activities designed by the agent. In this study the beneficiary of quality of ART service was the end user.

The 'framework' is presented as the context or environment in which activities takes place. This provides for living experiences and it applies to the public health facilities. In this study the perceptions of end users were explored in public health facilities where ART services were provided. These were subjective and were dependent upon the dynamic environment in which they were encountered (Moleki 2008:29).

The 'dynamics' provide the energy source or the motivating factors for quality of ART service provision. The dynamics in this study included the six elements of Wagner's chronic care model: delivery system design, self-management support, decision support, clinical information systems, community resources, and health care organisation. Good performance of the dynamics results in good end user satisfaction, efficient health services and good health outcomes.

The 'procedures' are the techniques or protocols that guide the activities (Moleki 2008:30). In this study the procedures were the end user focused best practice guidelines for ART service provision. Figure 1.1 shows the conceptual framework illustrating end user focused quality of ART [(adapted from a combination of Wagner's chronic care model (Singh \& Ham 2006:5) and Dickoff et al's Survey List (Moleki 2008:30)]. 


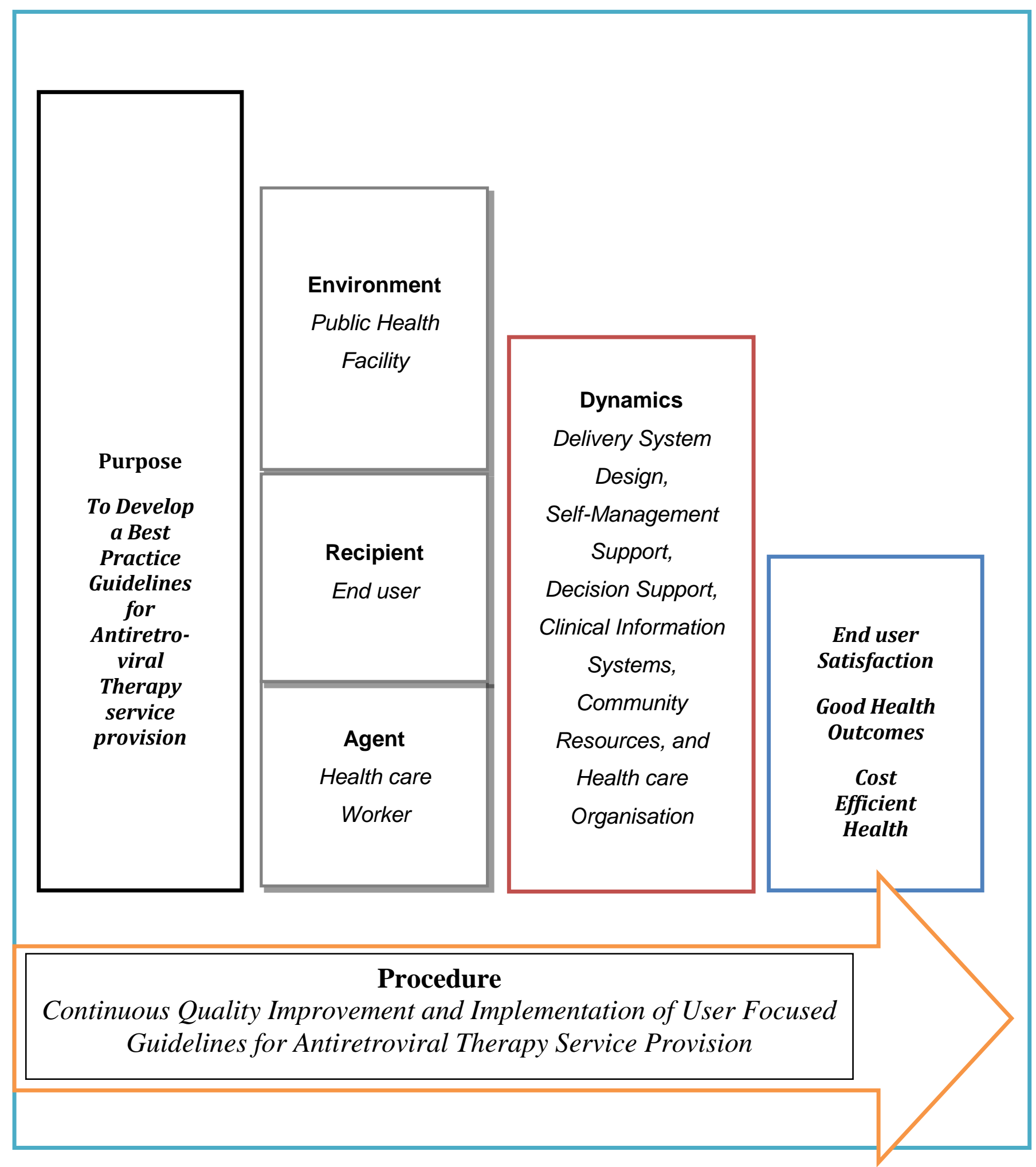

Figure 1.1 Conceptual framework illustrating end user focused quality of ART service provision

(Adapted from a combination of Wagner's chronic care model (Singh \& Ham 2006:5) and Dickoff et al's Survey List (Moleki 2008:30) 


\subsubsection{Theoretical framework for providing quality antiretroviral therapy and other definitions}

\subsubsection{Wagner's chronic care model}

Wagner's chronic care model was used as the theoretical framework for this study. The CCM identifies the essential elements of a (local) health care system, which encourages end user focused, high-quality ART service provision as chronic disease care (Minkman, Ahaus \& Huijsman 2007:91). The model is based on evidence-based change concepts and responds to the need for a continuous quality improvement model that fits ART service provision. The model focuses on linking informed, active PLHIV with proactive teams of professionals. Minkman et al (2007:91) report that the CCM has a number of extras relating to end user safety, cultural aspects of care, care coordination, and the Institute of Medicine's dimensions of good care, which includes safe, effective, timely, end user-centered and efficient care. It acknowledges that a substantial portion of chronic care takes place outside formal health care settings and suggests that six elements are of central importance in initiatives to improve chronic care: delivery system redesign; end user self-management; decision support; clinical information systems, community resources; and the health care organisation/health care system (Singh \& Ham 2006:5).

According to Singh and Ham (2006:5), and Wielawski (2006:5), CCM is the best known framework for care of people with chronic illnesses such as PLHIV. In fact, most chronic care policies and guidelines in developed countries now draw on this model to some extent (Singh \& Ham 2006:5). To elaborate on this assertion, Minkman et al (2007:91) conducted a systematic review of literature on the Malcom Baldrige Quality Award (MBQA) criteria, the European Foundation of Quality Management (EFQM) excellence model (Excellence award model) and the Chronic Care Model. The focus of their systematic review was the empirical evidence for improved performance by the implementation of interventions based on these models. The results indicated that no Excellence Award Model studies with controlled designs were found. For the CCM, one systematic review, one meta-analysis and six controlled studies were included. Although all the conclusions on CCM were drawn in the United States (US) settings for specific end user groups, there is some evidence that implementing interventions based on the 'evidence-based developed' CCM may improve process or outcome performance 
(Minkman et al 2007:96). Therefore, developing an end user focused best practice guideline for ART service provision should improve performance in the provision of quality ART services that meet end user needs and expectations.

While there is evidence that single or multiple components of the CCM can improve quality of ART service provision, clinical outcomes, and health care resource use, it remains unclear whether all components of the model, and the conceptualisation of the model itself, is essential for improving chronic care. The thrust of the CCM is on the evidence-based directions for each element, but extensive research on the effects of the model as a whole on improved performance remains limited and comes mainly from self-reported, uncontrolled studies (Minkman et al 2007:91).

Details of the CCM will be presented in chapter 2 of this thesis.

\subsubsection{Other definitions}

\section{Perceptions}

The Advanced Learners' Dictionary (2000:864) defines perception as an idea, a belief or an image you have as a result of how you see or understand something. Chow et al (2009:436) assert that perception is purely subjective, as the perception that end users have of ART service provision affects their expectations greatly, and in turn, their satisfaction. Different end users have differing perceptions of ART service provision, which has been shown to predict overall end user satisfaction (Chow et al 2009:436). Chow et al (2009:440) argue that several biases based on psychological theories may influence perception of satisfaction (quality).

First, the cognititive consistency theory implies that an end user is more likely to perceive and respond positively to satisfaction questions to justify their own time and effort spent obtaining treatment. Second, the Hawthorne effect implies that the very action of perceiving and measuring the level of satisfaction increases the apparent concern of the health care worker, thus improving satisfaction levels. Thirdly, social desirability bias indicates that end users give positive responses because they feel that these are more acceptable to satisfy researchers. Fourthly, ingratiating response bias implies that end users give positive responses to try and ingratiate themselves to health 
care workers, especially when anonymity is suspect. Similarly, Chow et al (2009:440) inferred that end users may be reluctant to provide negative feedback in fear of prejudice from the health care worker. Fifthly, self-interest bias cause end users to perceive and provide positive responses, because they believe this will allow the ART programme to continue running, which is in their best interest (Chow et al 2009:441).

In this study, perception refers to the views and opinions of end users regarding quality of ART service provision in public health facilities in Nigeria. The subjectivity and biases inherent in 'perception' was addressed through confidence building of research respondents and conduct of data collection as close as possible to the time of the health care encounter. The researcher recognised that the greater the duration of time between health care service use and the focus group interviews, the greater the chance of recall bias, changes in perceptions or appreciation of care, and the possibility of end users overlooking aspects that bothered them at the time of care (Chow et al 2009:440).

\section{Anti-retroviral therapy}

According to the WHO (2006:9) ART refers to the delivery of antiretrovirals as part of care interventions, including the provision of co-trimoxazole prophylaxis, the management of opportunistic infections and co-morbidities, nutritional support and palliative care. In this study, ART refers to the prescription and taking of antiretrovirals, at the correct time intervals, on a daily basis of one's entire life. Also laboratory monitoring and management of opportunistic infections, adhering to food prescriptions, and taking generally good care of one's health. ART also involves keeping follow-up clinic visits and maintaining available supplies of antiretrovirals.

\section{Anti-retroviral drugs}

Tindyebwa, Kayita, Musoke, Eley, Nduati, Coovadia, Bobart, Mbori-Ngacha and Kieffer (2005:137) refer to antiretrovirals as drugs that suppress HIV replication and therefore prevent disease progression. Antiretrovirals are medications for the treatment of infection by retroviruses, primarily HIV. The drugs do not kill the virus. However, they slow down the growth of the virus. When the virus is slowed down, so is HIV disease. When several such drugs, typically three or four, are taken in combination, the approach is known as highly active antiretroviral therapy (HAART). In this study, ARVs refer to 
medications that reduce the morbidity of people living with HIVIAIDS (PLHIV) and improve their quality of life.

\section{End user}

The Advanced Learners' Dictionary (2000:383) and Free Dictionary [S.a.] defines end user as the person who actually uses a product (or service) rather than one who makes or sells it, especially, the ones for whom the product or service has been designed. In this study, end users refer to the ultimate consumers (end users) of antiretroviral therapy services in public health facilities in Nigeria. These are people living with HIVIAIDS (PLHIV) and attending ART services in public health facilities in Nigeria.

\section{Best practice}

The Advanced Learners Dictionary (2000:912) describes best practice as a way of doing something that is the usual or expected way in a particular organisation or situation. In this study, best practice refers to a standard way of providing quality ART services that meets end users' requirement for satisfaction with care. The best practices are set out as instructions in the form of a guideline. See operational definition of guidelines, below).

\section{Quality}

WHO (2004:3) defines quality as the totality of features and characteristics of an entity that bears on its ability to satisfy a stated or implied need. Describing quality from the perspectives of end users, Liu and Wang (2007:267) defined quality nursing care as having nurses who were concerned about end users and demonstrated caring behaviour, were competent and skilled, communicated effectively with them, and taught them about their care. Chow et al (2009:439) view end user's perspective of quality as any aspect of an end user's health status that comes directly from the end user (that is, without the interpretation of the end user's responses by a health care worker or anyone else). Gill and White (2009:15) postulate that perceived health service quality is adopted as an inter-changeable terminology with end user satisfaction. Satisfaction can therefore be defined as the extent of an individual's experience compared with his or her expectations (Udoh et al 2011:220). In other words, Udoh et al (2011:220) 
elucidated that end user satisfaction is related to the extent to which general health care needs and condition-specific needs are met. Liu and Wang (2007:266) defined satisfaction as the psychological state that results from confirmation or disconfirmation of expectations with reality. End user satisfaction with nursing care was defined as the degree of congruency between an end user's expectations of ideal nursing care and her or his perception of the actual nursing care received (Liu \& Wang 2007:266). End user perceived quality may be judged as performance of health care, based on end users' expectations of the health care system and of how they would like to be treated (Hopkins, Loeb \& Fick 2009:927). Chow et al (2009:438) summarised the components of perceived end user satisfaction into three broad areas: affability, accessibility, and ability (3-As). The affability or interpersonal manner of health care worker is often thought of as the principle component of end user satisfaction. Accessibility and availability include aspects such as physical access to health facilities, efficiency of health facility appointment systems, waiting times, waiting lists, and home visits. The technical quality of care or ability of the health professional is usually thought of by health care workers as being the most important of care; yet, it is the least important to the end users. This study will particularly focus on the first two components; affability and accessibility.

In this study, quality refers to end user satisfaction, or meeting the requirements of end users through understanding their perceptions on their satisfaction (quality) of the ART services rendered.

\section{Health care quality}

Creel et al (2002:2) defined health care quality as offering technically competent, effective, safe care that contributes to the end user's well-being. In this study, health care quality refers to end users' perceptions of their satisfaction (quality) with ART service provision, as may be influenced by social and cultural concerns, with significant emphasis on the human aspects of care.

\section{Total quality management}

Total quality management (TQM) is generally interchangeable in the health care quality literature with continuous quality improvement (CQI). WHO (2004:3) defined TQM as an 
organisation-wide approach, aimed at continuously improving overall performance, while allowing integration of other quality assurance approaches to quality such as quality control and accreditation. TQM is based on the whole system and on the participation of customers, clients and society (WHO 2004:3). Francois, Peyrin, Touboul, Labarere, Reverdy and Vinck (2003:52) on the other hand, assert that quality management is said to be total when it becomes an absolute priority of the organisation, outweighing other elements of management such as financial or budget management or management of human resources.

For the purpose of this study, TQM refers to a comprehensive system of implementing scientific and evidence based health care improvement methods to gain knowledge and control over ART service provision, to standardise health care processes, improve end user satisfaction and hopefully, reduce cost of health services.

\section{Guidelines}

The Advanced Learners' Dictionary (2000:528) defines guidelines as rules or instructions that are given by an official organisation telling you how to do something, especially something difficult. In this study, guidelines are used as a set of instructions necessary to provide end user focused quality ART services. The word is used along with "best practice" to denote best practice guidelines, which denotes standard sets of instructions telling health care workers how to provide end user focused ART service that ensures user satisfaction.

\subsection{RESEARCH DESIGN AND METHOD}

\subsubsection{Research design}

A descriptive and explorative qualitative research design was used in this study. The use of this design in this research is justified on the basis of the flexibility of the design in promoting descriptions of the perceptions of end users concerning quality of ART services provision in Nigerian public health facilities.

The design will be elucidated in chapter 3 of this thesis. 


\subsubsection{Research setting}

The study was conducted among PLHIV accessing ART in six public health facilities, located in each of the six geopolitical zones of Nigeria. These health facilities receive support from international technical assistance organisations to implement quality comprehensive ART programmes. Consequently, the six public health facilities selected for the study were suitable for this study attempting to explore and describe the perceptions of end users concerning the quality of ART in Nigerian public health facilities. Further details on the research setting will be discussed in chapter 3 of this thesis.

\subsubsection{Research population}

The research population of interest to the study comprised all end users attending ART services in Nigerian public health facilities over a period of 5 years. However, the costs and time required to reach such a population were beyond the scope of the researcher. Consequently, the target population for this study included end users in six public health facilities, representing each of the six geopolitical zones in Nigeria. The accessible population included all end users attending ART services at the six participating study sites in Nigeria. The concept of the research population will be explained in chapter 3 of this thesis.

\subsubsection{Data collection procedure}

Focus group discussions were used to explore the perceptions of end users concerning the satisfaction with (quality) of ART in Nigerian public health facilities. Focus group discussions were preferred in this study to elicit information from the respondents until the point of saturation had been reached. The data collection procedure will be discussed in chapter 3 of this thesis.

\subsubsection{Data analysis}

The analysis of qualitative data began in the field, during data collection, using unstructured focus group discussions. The framework approach for qualitative data 
analysis and Weft QDA version 1.0.1 software were used, because they reflected the original accounts of the perceptions of the participants and observations made by the researcher of the participants (end users attending ART). The concept of data analysis will be discussed in detail in chapter 3 of this thesis.

\subsubsection{Ethical considerations}

The researcher ensured strict compliance with ethical standards, relevant to protecting the rights of the respondents, institutions where data were collected and scientific integrity were maintained throughout the study. Approval was sought and obtained from both the health authorities in Nigeria (National Research Ethics Committee) and the Department of Health Studies, University of South Africa (Unisa) before data collection commenced. Details of these will be presented in chapter 3 of this thesis.

\subsection{SCOPE AND LIMITATIONS OF THE STUDY}

Six public health facilities implementing comprehensive ART services in Nigeria, participated in the study. Consequently the results of this study might not be generalisable beyond these six participating ART sites.

Only the perceptions of the end users were studied. Health care workers, end user caretakers and end users' relations might have different perceptions about the quality of ART services in the public health facilities. End users who attend private or faith-based health facilities might also have different perceptions. However, due to time limitations the perceptions of caretakers, health care workers and end user relations were not obtained. Permission to conduct focus group discussion took several months to be granted by the National Research Ethics Committee, Nigeria. Details of the limitations of the study will be presented in chapter 6 of this thesis.

\subsection{STRUCTURE OF THE THESIS}

This thesis is presented in seven chapters, organised in the following order:

Chapter 1: Orientation of the study, covering the background information about the research problem, definitions of key terms, statement of the research problem, aim of 
the study, significance of the study, foundations of the study, research design and method, scope of the study and the structure of the thesis.

Chapter 2: Literature review, addressing perceptions of end users concerning quality of ART.

Chapter 3: Research design and methods, including sampling, population of the study, data collection approach and method, data analysis, trustworthiness of the study and ethical considerations of the study.

Chapter 4: Analysis, presentation and description of the research findings.

Chapter 5: Discussion of research findings.

Chapter 6: Conclusions, recommendations and limitations are presented in the final chapter.

Chapter 7: End user focused best practice guidelines for provision of quality antiretroviral therapy in public health facilities.

\subsection{CONCLUSION}

This chapter provided the background information related to the focus of the study, which highlights the core issues that stimulated the researcher's interest to investigate on this topic. The Nigeria national guidelines for ART service provision, mainly provider focused was noted to have failed to address quality of ART service provision from the perspectives of the end users. This therefore compromises good care, which is otherwise elucidated as safe, effective, timely, end user focused and efficient ART care. In other words, the Nigeria national ART guideline lacks the complement of end user focused guidelines which addresses the needs and expectations of the end users.

Therefore, to meet the increasing needs of end users, and add to the body of knowledge regarding ART service provision, the existing guidelines for ART service provision, mainly provider focused, must be complemented with end user focused 
guidelines that address the needs and expectations of end users in Nigerian public health facilities. In order to achieve the purpose of the study, the researcher sought to explore and describe the perceptions of end users concerning the quality of ART service provision in Nigerian public health facilities. A conceptual framework, adapted from Dickoff et al's survey list was described alongside a theoretical framework on Wagner's chronic care care model. These frameworks will be used in the development of the end user focused best practice guidelines for ART service provision.

The next chapter (chapter 2) will present a literature review on the perceptions of end users concerning quality of ART service provision. 


\section{CHAPTER 2}

\section{LITERATURE REVIEW}

\section{$2.1 \quad$ INTRODUCTION}

This chapter entails the critical analysis of the reviewed literature on quality of antiretroviral therapy in public health facilities in Nigeria and the perceptions of the end users. Steps adopted in conducting the literature review included mobilisation and collection of scientific print materials (research articles) relevant to the research topic, from electronic data bases, institutional and personal libraries. This was followed by scanning through all the literatures, marking and coding those most relevant to the research topic, for more detailed reading. During detailed reading, relevant sections of the literature were marked; using highlighters of different colors to denote their relevance to each research objective. All marked sections of the literature were compiled, drawn together based on common features and critically appraised to give concrete meanings that relate to the research topic. Relevant references were compiled for addition into the list of references.

The chapter further discusses the theoretical framework that was used as the underlying departure of this study, which is the Chronic Care Model. The presentation in this chapter includes:

- Wagner's Chronic Care Model

- $\quad$ Perceptions of end users concerning quality of ART service provision

\subsection{WAGNER'S CHRONIC CARE MODEL}

Chronic Care Model (CCM) was developed by Edward Wagner and his team in the United States in 1998 and implemented in various settings to improve the quality of care of people with chronic illnesses (Singh \& Ham 2006:5). Wielawski (2006:5) reports that Wagner's decision to develop the CCM was informed by the assumptions that the typical clinical care setting such as those providing antiretroviral therapy was set up to 
respond to acute illness rather than to anticipate and respond proactively to end users' needs. People living with HIVIAIDS, however, need the latter approach in order to avoid acute episodes of illness and debilitating complications. In addition, chronically ill end users are not sufficiently informed about their conditions, nor are they supported in selfcare beyond the clinical setting. Finally, physicians are too busy to educate and support PLHIV to the degree necessary to keep them healthy (Wielawski 2006:5).

The need for health care quality and quality of ART service provision is increasingly focusing on the centrality of the end user. Minkman et al (2007:91) report that end usercentered care focuses on the total needs of end users, not only on the services provided by one professional or organisation; thereby, sustaining seamless integrated care during the whole care process. For the public health facilities, this requires horizontal coordination, collaboration with other organisations and community partners or service integration. Partners in the care chain and the functioning of the care chain or care network as a whole contribute to total quality management of ART care. To facilitate the improvement of health care quality and performance of ART service provision, the CCM was selected as the framework of choice. The integrated quality management model, CCM, was selected on the basis of multiple criteria, as elucidated by Minkman et al (2007:91). Firstly, the CCM consist of multiple 'enablers' of good quality care (for instance, leadership or delivery system design). Enablers cover the processes, structure and mean values of a health facility.

Secondly, the CCM focusses on multiple performance dimensions of multiple stakeholders (for instance, organisational performance). Lastly, the CCM assumes dynamic relationships between improved performance and implementation based on its enablers. It is therefore frequently used as framework for local end user-focused improvement or national collaborative improvement programmes (Minkman et al 2007:91). According to Singh and Ham (2006:5) and Wielawski (2006:5), CCM is the best known framework for care of people with chronic illnesses such as PLHIV. In fact, most chronic care policies and guidelines in developed countries now draw on this model to some extent (Singh \& Ham 2006:5). The model focuses on linking informed, active PLHIV with pro-active teams of professionals.

The CCM was compared against similar models in order to justify its application to this study. Findings of a systematic review conducted by Minkman et al (2007:102) indicate 
that CCM studies merely looks at end user groups and clinical measures. This is a better 'fit' to bio medically oriented research paradigm, such as investigating the perceptions of end users concerning quality of ART service provision in public health facilities. Whereas other models such as the EFQM and MBQA models are mainly used as management tools at the strategic level, the CCM is mainly used as a tool to optimise care for a specific end user group (such as PLHIV) at the more operational or process level. Minkman et al (2007:102) added that with the attention to costs and efficiency in current ART service programming, it is surprising that only a few CCM studies measured financial performances. Moreover, measures of worker satisfaction (the care team) and end user perceptions/judgments concerning care were also often limited. Singh and Ham (2006:5) acknowledges that a substantial portion of chronic care takes place outside formal health care settings and suggests that six elements are of central importance in initiatives to improve chronic care: community resources; the

health care system; end user self-management; decision support; delivery system redesign; and clinical information systems. The components of the model are based on research evidence.

\subsubsection{Elements of the chronic care model}

The six key principles/elements of this model, whose coordination is necessary for highquality ART management, include (Wielawski 2006:6; Singh \& Ham 2006:5):

\subsubsection{Delivery system design}

This refers to delivering effective, efficient care and self-management support. To move from the one-on-one doctor/end user relationship to team-work, the clinical members of staff need defined roles and tasks. Follow-up with end users is essential so that they feel supported in self-management efforts outside the medical office. Key activities could include care management roles clarification, team practice, care delivery/coordination, proactive follow-up, planned visits, and visit system change.

\subsubsection{Self-management support}

This refers to empowering and preparing end users to manage their health and health care. Clinicians should set a tone of collaboration with chronic end users and encourage 
their participation in setting goals and fine-tuning treatment. Other aspects of selfmanagement support could include end user education, activation/psychosocial support, self-management assessment, self-management resources and tools, collaborative decision-making with end users and making guidelines available to end users.

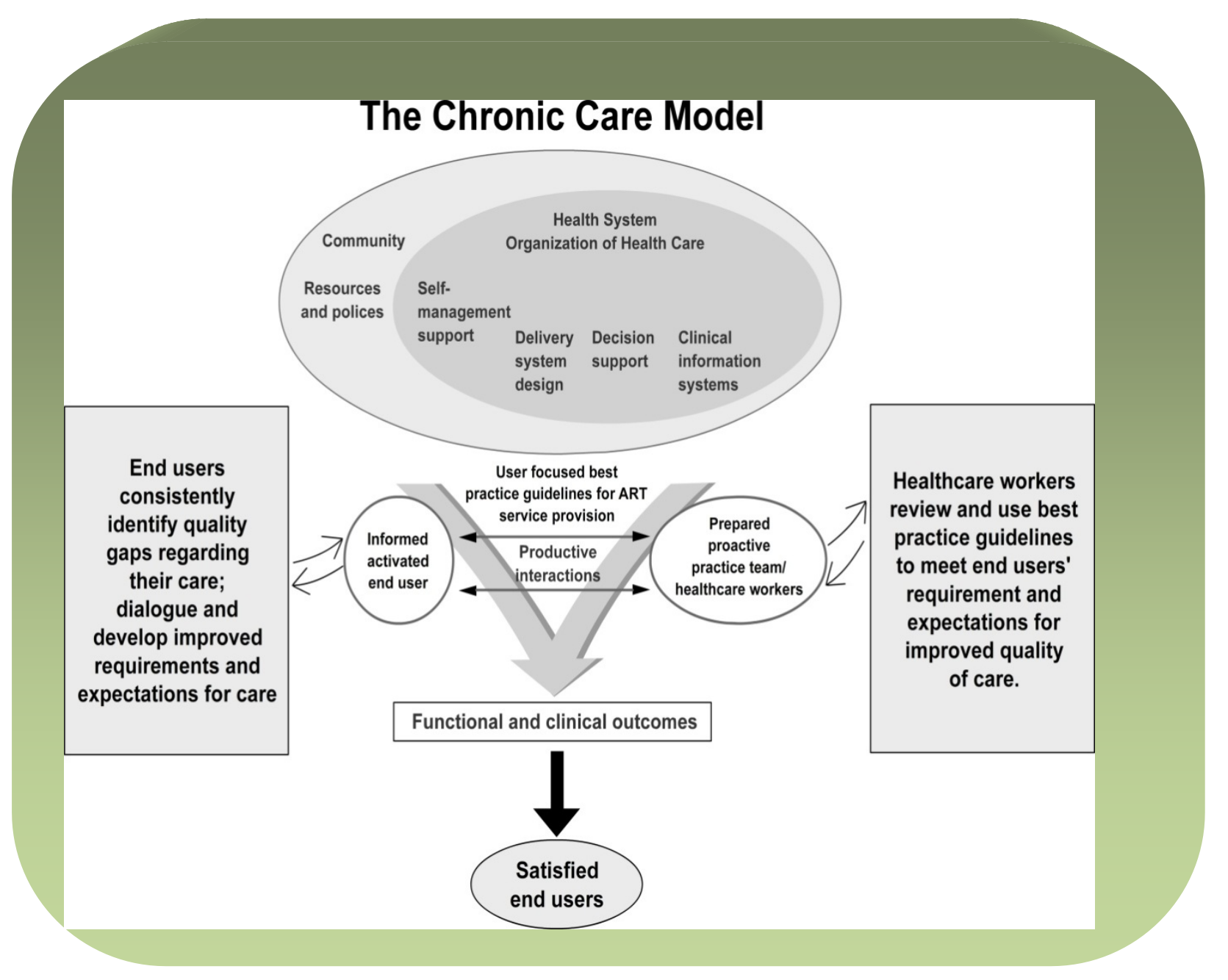

Figure 2.1 Wagner's Chronic Care Model

(Adapted from Singh \& Ham 2006:5)

\subsubsection{Decision support}

This refers to promoting care that is consistent with research evidence and end user preferences. To link treatment to research evidence, clinicians must have explicit 
guidelines, whether the question is scientific (drug use) or psychosocial (how best to motivate end users to adhere to ART).

\subsubsection{Clinical information systems}

This refers to organising end users and population data to facilitate efficient and effective care. Computers can efficiently deliver disease management information, including care guidelines, test results, and even pop-up reminders about individual end users. They also facilitate so-called population studies, of say, all PLHIV in the health facility; the care team can measure their performance against quality benchmarks.

\subsubsection{Community resources}

This refers to mobilising community resources to meet the needs of people with longterm conditions such as PLHIV. Health care providers should identify existing programmes in the community and encourage end users to participate.

\subsubsection{Health care organisation/health system}

This refers to creating a culture, organisation, and mechanisms that promote safe, high quality care. Health care organisations must make excellence a priority and pursue it visibly. Essential to achieving this is top management support, provider participation and open communication on error and failings as well as strategies for coherent system improvement and spread.

By integrating these elements into ART, Wielawski (2006:6) laments that health facilities are better able to stay on top of clinical responsibilities while helping end users become active participants in their care. The result is win-win all around, he says. End users are healthier, providers are cheered and motivated by evidence of a job well done, and the health care system saves money.

\subsubsection{Justification for using the chronic care model in this study}

The CCM was selected as theoretical framework for this study due to available evidence that implementing interventions based on the 'evidence-based developed' 
CCM will improve quality, process or outcome performances of ART service provision in public health facilities (Minkman et al 2007:90). Minkman et al (2007:90) added that CCM has features that look at the total needs and expectations of the end user and ensures all aspects of the needs are well integrated and coordinated into a whole. The CCM ensures that end users achieve the right information regarding their care, in the right setting, using the right technologies that are readily available, by the right personnel (including through self-care), delivered at the right time, on the right site(s), given the right amount of care, delivered appropriately/right care, and the right fit to purpose of the end user (Wakefield, Ward \& Wakefield 2007:104). Although the study looks at ART service provision in the public health facilities only, the use of CCM model as the framework for this study will allow for integrating and meeting the total needs of the end user, some of which may be in the community. To achieve this user-focused package, the results of this study and the eventual best practice guidelines for quality ART service provision will be presented in line with the six elements of the CCM.

Within each element of the CCM, the researcher will provide rich information, derived from the findings of this study, to describe how the needs and expectations of the end user will be met. The thick descriptions under each element of the CCM will be action oriented, to enable health care workers to translate the best practice guidelines into improving the quality of ART service provision. It is anticipated that with the 'evidencebased developed', end user focused CCM, the eventual best practice guidelines will be user-focused and practical oriented, with features that ensure seamless, coordinated and integrated care throughout the whole care process.

To corroborate the strengths of CCM as the best framework for this study, Minkman et al (2007:90) reported a systematic review conducted from 1995 to May 2006 to find empirical evidence for improved performance by the implementation of interventions based on MBQA, EFQM and the CCM. A search in the Pubmed, Cochrane, and ABIdatabases showed that no Excellence Award model (EFQM, MBQA) studies with controlled trials were done. For CCM, one systematic review, one meta-analysis and six controlled studies were included, and all the studies used more solid designs and methods than the MBQA and EFQM. Singh and Ham (2006:7) also confirmed that the CCM was developed based on perceptions of best practices and high quality evidence of meeting end users' needs and expecations. 
For an example, a Cochrane systematic review of hundreds of studies suggested a synergistic effect when individual interventions (components of the model) were combined (Singh \& Ham 2006:7). Observational studies reported better processes, outcomes, or costs in individual health facilities adopting the CCM, but such studies do not tell whether the model was more effective than others (Singh \& Ham 2006:7). Thus, a formal evaluation program was set up in the US to assess the effects of the CCM on end users. Singh and Ham (2006:7) suggested that successful implementation of the CCM can lead to better processes and outcomes of care, including end user satisfaction, clinical outcomes, and costs. However, four components of the model were most likely to be associated with sustained change: organising practice teams; collaborative decision-making with PLHIV; encouraging provider participation in improvement efforts; and de-emphasising traditional end user education.

The researcher applied all six components of the CCM to guide the focus of the study, and achieved overall quality improvement in developing the best practice guidelines for ART service provision. Each of the six components of the CCM formed building blocks and dynamics for developing the end user-focused best practice guidelines for ART service provision in public health facilities. It is therefore envisaged that once the developed best practice, user focused guidelines are used to provide ART services in public health facilities, the quality of ART service provision will be improved, end users will be satisfied with ART services and good clinical outcomes will be achieved at lower costs. Overall, the end user will be satisfied with care.

\subsubsection{Exclusion process for related models}

Many models focus on service delivery approaches and case management, rather than a broader approach taking into account multifaceted components like the broad frameworks. Singh and Ham (2006:30) compared the impact of five models (broad frameworks). The researcher leveraged on this comparism in order to exclude some models to determine one that will be used for this study. The broad frameworks compared in this study include: Chronic Care Model, Innovative Care for Chronic Conditions Model (ICCCM), Public Health Model, Continuity of Care Model, and the National Health Service and Social Care model. 
There is limited high quality evidence about the impact of these models. Although components of the CCM have been studied extensively and a detailed evaluation has been undertaken in the US, it is still unclear whether this model is any more effective than others. This is largely because other models are not well conceptualised or described. Singh and Ham (2006:30) indicate that there is evidence that improvement programmes which aim to implement the CCM can have a sustainable impact on quality of care and some clinical and resource outcomes.

Singh and Ham (2006:9) assert that the ICCCM focuses on improving care at three different levels: micro level (individual and family), meso level (healthcare organisation and community), and macro level (policy). Like the CCM, there is evidence that specific components of the ICCCM can improve some processes and outcomes. However, Singh and Ham (2006:9) report that no review, trial, or observational studies have explicitly attempted to assess the effectiveness of the ICCCM and no rigorous evidence has been noted about the value of its policy focus. Based on sub optimal reports of its effectiveness, the ICCCM is determined to unsuitable for use as theoretical framework in this study.

Another model compared by Singh and Ham (2006:10) and leveraged in this study was the Public Health Model. The underlying principle of this model is that in order to impact on the burden of chronic conditions, three levels of intervention must be in place. These include population-wide policies, community activities, and health services. This model is more interested in population wide (public health) care of chronic diseases, therefore, not suitable for individual based, user focused care as required in this study. Additionally, although there are evaluations of individual public health initiatives, Singh and Ham (2006:10) report that there is no study of the implementation of the Public Health Model and no evidence of its effectiveness. This model was therefore determined unsuitable for use as theoretical framework in this study.

The Continuity of Care Model outlines how chronic conditions develop in response to risk factors in the community and suggests points at which to target prevention efforts, medical intervention, treatment and rehabilitation, and palliative care (Singh and Ham 2006:11). This model tracks chronic care from the general population; through people who develop one or more long-term conditions following exposure to risk factors; through to people who have terminal disease. The model suggests the need for different 
prevention schemes, treatment, rehabilitation, and palliative care at varying stages of the disease pathway. Although there are evaluations of individual interventions that prioritise continuity, Singh and Ham (2006:11) report no high quality studies of the implementation of the Continuity of Care Model, and thus, no evidence of effectiveness. Against this background, this model was determined unsuitable for use as theoretical framework in this study.

Of all the models compared above, Singh and Ham (2006:30) report that only the CCM had evidence that its components can improve quality of care and resource use. There was however, not comparative evidence about whether the CCM is better than other frameworks. Nevertheless, when compared to other broad frameworks, only the CCM had better evidence of effectiveness in improving quality of care. Therefor e, the CCM was determined as the best match for this study.

\subsubsection{Limitations of the chronic care model}

Singh and Ham (2006:8) report that while broad chronic care programmes may improve health professional's adherence to evidence-based standards of care, there was no strong evidence about which components of these programmes may impact on the quality of care provided. Nor did they find evidence of a direct link between broad chronic care programmes and reductions in mortality, improvements in quality of life, or cost-effectiveness.

In summary, while there is evidence that single or multiple components of the CCM can improve quality of care, clinical outcomes, and health care resource use, Singh and Ham (2006:8) report that it remains unclear whether all components of the model, and the conceptualisation of the model itself, is essential for improving the chronic care of AIDS.

\subsection{PERCEPTIONS OF END USERS CONCERNING QUALITY OF ANTI- RETROVIRAL THERAPY}

According to Creel et al (2002:3), the end user's perception of the quality of care emphasises the method of choice and availability, respectful and friendly treatment, privacy and confidentiality, service providers' professional competence, information and 
counselling, convenient hours and acceptable waiting times, and affordability. Three elements can help end users feel well treated: face-to-face communication; skilful providers who show end users that they care about their work; and consideration of how end users' needs, fears, and reactions may be perceived differently by health care providers (Creel et al 2002:3).

Creel et al (2002:7) explain that improving quality of care for end users using the chronic care model means understanding their cultural values, previous experiences, and perceptions of the role of the health system, and then bringing health service providers and the community together to map out a shared vision of quality. Similarly, Creel et al (2002:7) say enhancing health care for providers requires identifying their motivations, addressing their needs (including general administrative and logistical support from the health care system), and helping them to better understand and address end users' concepts of quality.

The Health Care Improvement Project (HCIP) (2008:1) offers modern quality improvement approaches for overcoming common barriers to health care quality, even in the context of weak health systems facing severe material and human resource constraints. Quality improvement (QI) methods improve processes of care and are based on four principles: (i) understanding and focusing on health service user needs; (ii) understanding how processes of care function within the system; (iii) using data to measure results; and (iv) engaging teams of managers, service providers, and community stakeholders in improvement.

HCIP (2008:1) notes that the focus on end user satisfaction is central to the quality improvement framework, which perceives the primary purpose of health services as being to meet the needs and improve the health and well-being of the end users who use them. The emphasis on systems and processes of care is also central, since poorly

designed systems generate inefficiency and waste, poor health care quality, and negative health outcomes.

\subsubsection{Perceptions of end users concerning quality of antiretroviral therapy}

Page-Shipp, Charalambous, Roux, Dias, Sefuti, Churchyard and Grant (2007:383) investigated perceptions and attitudes to directly observe antiretroviral therapy (DOT 
ART) among HIV infected adults attending a workplace HIV care programme in South Africa. The study concluded that ART by DOT was not an immediately popular concept with end users, primarily because of a desire to retain responsibility for their own treatment.

Chiegil (2010:50) reports a study conducted in Taraba State of Nigeria. The study sought to explore the factors relating to quality of care and their influence on antiretroviral adherence among end users in Nigeria. The factors that kept end users satisfied with care include clean and attractive service environment and courtesy of doctors, nurses and other health care. The following factors devastated end users from achieving their expectations for quality care: frequent stock out of cotrimoxazole and multivitamins; non-routine laboratory tests; suboptimal skilled health care workers; high fees for services and disharmony between health facility and community based support group members.

End users are increasingly demanding improved quality of health services, while supply remains sub-optimal and factors of quality continues to be viewed differently by end users. Using a structured questionnaire Hasin, Seeluangsawat and Shareef (2001:7) identified the following quality factors by interviewing end users attending the out-patient department (OPD) and in-patient department (IPD) in Muang Petch Thonburi Private health facility in Thailand. Findings by authors showed that each end user listed many factors, based on which the following factors were concluded:

a) Cleanliness.

b) Service of doctor (quick response to request for service, intention and attention of doctors in listening to an end user, diagnosing method, treatment explanation, courtesy).

c) Service of nurses (quick response to request for nursing service, help and attention of nurses, courtesy).

d) Service of officers, i.e. staff (quick response to request for financial service, quick response to request for other services, courtesy of finance staff, courtesy of pharmacy staff, courtesy of receptionist, courtesy of end user handling officers, availability and reception of information).

e) Other services (service rate, food and test). 
Hasin et al (2001:6) suggest that the main factors, which affect end user satisfaction in health care facilities/provision, are: doctor, drug, diagnosis, duration, distance, affordability, prompt service, etc. According to the authors, these are the factors that comprise the health care quality system, and the higher the efficiency of the quality system, the more will be the satisfaction of end users.

Study results highlighting the influence of infrastructure/environment on end user satisfaction: Pietrovsk and Dall'Agnol (2006:630) were involved in a qualitative study performed at a haemodialysis clinic in the interior of Parana to look at significant situations of chronic renal end users. Results of this study indicated aesthetics and cleanliness of the environment as factors that interfere in end user satisfaction (Pietrovsk \& Dall'Agnol 2006:630). Gaioso and Mishima (2007:1) on the other hand, noted that dissatisfaction in their own study was predominated in terms of environment and organisational accessibility, impairing care longitudinality and continuity.

Medeiros et al (2010:402) conducted a study to evaluate end users' perception of a basic health unit (BHU) in a medium-sized Brazilian city, analysing their satisfaction and participation in social control. Results of the study indicated poor infrastructure (physical area) in the BHU (Medeiros et al 2010:402). In another study to compare the quality of family planning services delivered in public and private health facilities in Kenya, Agha and Do (2009:87) report that private facilities were superior to public sector facilities in terms of physical infrastructure and the availability of services.

Study results highlighting the influence of affability on end user satisfaction:To explore and establish the predictors that exert the greater influence on the level of satisfaction with the service offered in a University Hospital in Chile (Hospital Clinico de la Pontificia Universidad Catolica de Chile), Castillo, Dougnac, Vincente, Munoz and Rojas (2007:696) maintain that the factor with the greatest predictive power was the relationship between the end users and health care workers (nursing staff). Other factors that emerged as important were communication, medical attention and room conditions (Castilo et al 2007:696). In a study to evaluate the satisfaction of the end users with health services in northwest Parana State, 68 per cent of the respondents answered that their health care workers were good, while 58 per cent said the health facilities were great. In a study conducted to assess users' acceptability of the care offered and delivered by Family Health teams in Ribeirao Preto, Sao Paulo, Brazil, the 
end users indicated considerate and affective interpersonal relations as one point of satisfaction and one of the aspects that differentiates health care (Gaioso \& Mishima 2007:1). Private providers were better at managing interpersonal aspects (affability) of care (Agha \& Do 2009:87). Regmi and Madison (2010:44) evaluated end user satisfaction with the new second-trimester abortion services in Nepal. Satisfaction in this study was found to be high for characteristics associated with counselling and the caring attitude of the health care workers (Regmi \& Madison 2010:44).

Mishima, Pereira, Matumoto, Fortuna, Pereira, Campos and Paula (2010:436) conducted a descriptive exploratory study to analyse end user satisfaction with the care received at a Family Health Unit in Ribeirao Preto, Brazil. The subjects of this study expressed satisfaction with the accessibility provided together with the caring attention given to them, marked by a team-user interaction that takes place in a friendly and patience manner (Mishima et al 2010:436). Mishima et al (2010:436) agree that although not totally satisfied, the majority of users would recommend the health service to someone due to its quality. In a similar study to assess the satisfaction of end users with the interdisciplinary care delivered in a clinic of secondary prevention of chronic kidney disease (CKD), Santos, Lima, Elias, Magacho, Oliveira, Fernandes, Carmo, Abrita and Bastos (2008:151) reveal that a great majority (95,5 percent) of the end users reported to be satisfied with the explanation given about their disease. Regarding the management of their disease, the end users considered important or very important the role played by health care workers (Santos et al 2008:151). Santos et al (2008:151) confirm that all end users would refer others with CKD to the same health facility. Cabrera-Arena, Bello-Parias, Jaime and Londono-Pimienta (2008:593) conducted a study to establish a quality baseline as perceived by health facility users, subject to intervention by the health-service restructuring, redesign and modernisation programme. Outcome of the study showed that quality was generally rated as good as perceived by end users in the health facility programme (Cabrera-Arena et al 2008:593). Cabrera-Arena et al (2008:593) added that the humanised treatment received from the health care workers was given the highest score regarding quality of attention received by the interviewees. To describe the user's opinion regarding access and quality care in health centers in Porto Alegre, Brazil, Lima, Ramos, Rosa, Nauderer and Davis (2007:12) reveal that users appreciated professional courtesy and quality care, and the bond established between them and the health care workers, which addressed appropriately the needs of the end users. To assess quality differentials and impact 
upon family planning (FP) service end users' satisfaction at public and private health facilities in three representative sub-Saharan African countries (Tanzania, Kenya and Ghana), Hutchinson, Do and Agha (2011:203) revealed that private health facilities appear to be of higher (interpersonal) process quality than public health facilities.

Technical quality is not necessarily higher in the three countries, though differentials are considerably larger at lower level facilities (clinics, health cernters, dispensaries) than at secondary health facilities (Hutchinson et al 2011:203). Hutchinson et al (2011:203) further infer that FP end user satisfaction appears considerably higher at private health facilities - both hospitals and clinics - most likely attributable to both process and structural factors such as shorter waiting times and fewer stockouts of methods and supplies. In a study to compare the satisfaction rating during obstetric sonography between missionary and public health facilities in Nigeria, Udoh et al (2011:222) report that end users were moderately satisfied with the friendly attitude of the sonographers in both public and missionary health facilties.

It is important to highlight that not all study reports showed good end user satisfaction with affability in health care services. In a study conducted by Mayeye, Lewis and Oguntibeju (2010:274), to assess adolescents' satisfaction with reproductive health services in Mdantsane Township, Eastern Cape Province, South Africa. Results indicated that satisfaction responses on accessibility and confidentiality of services, options available to participants as well as staff friendliness fell below the stated cut off point of 95 per cent (Mayeye et al 2010:274). Benazzi, Figueiredo and Bassani (2010:861) conducted a study to evaluate end users' satisfaction with ophthalmologic service provided in an urban center of the South of Brazil. The results showed that 36.3 percent of end users considered the ophthalmologic service as regularly terrible (Benazzi et al 2010:861). Regmi and Madison (2010:44) report that dissatisfaction was expressed regarding some aspects of their study, viz, delivery of care, especially, the lack of privacy and confidentiality and the absence of a support person from the patient's own family. These factors were highly regarded by the end users as necessary for their satisfaction with overall health care (Regmi \& Madison 2010:44). In a study by Yakong, Rush, Bassett-Smith, Bottorff and Robison (2010:2431), to describe rural women's perspectives on their experiences in seeking reproductive care from health care workers (professional nurses), major themes from women's stories include experiences of intimidation and being scolded; experiences of limited choices; receiving 
silent treatment, and experiences of lack of privacy. Women emphasised the importance of their relationships with health care workers (nurses) and the impact of these relationships on their health care-seeking (Yakong et al 2010:2431). In a study to analyse end user satisfaction based on the results of the World Health Survey, conducted in Brazil in 2003, Gouveia, Souza, Luna, Souza-Junior and Szwarcwald (2005:S109) espoused that multiple regression analysis showed that having experienced some type of discrimination (on the basis of gender, age, poverty, social class, skin colour, or type of disease) and being an exclusive user of the public National Health System involved a lower degree of users' satisfaction.

Chow et al (2009:438) re-iterates that affability or interpersonal manner of health care worker is often thought of as the principle component of end user satisfaction. Udoh et al (2011:221) hypothesise that the mode of health care delivery appears to be more important to end users than the actual care itself. This hypothesis agrees with Coulter (2005:1199) who infers that end users want health care workers who are good communicators and have sound, up to date clinical knowledge and skills. They also want health care workers who are interested and sympathetic, involve them in decisions, give them sufficient time and attention, and provide advice on health promotion and self care (Coulter 2005:1199). For an example, individuals who had received voluntary counselling and testing in Kisesa district in Tanzania resented the need to regularly travel to Mwanza, particularly because the health facility had a reputation as confusing and unfriendly to villagers (Mshana, Wamoyi, Busza, Zaba, Changalucha, Kaluvya \& Urassa 2006:655).

In conclusion, the literature of the perceptions of end users on quality indicated:

- $\quad$ courtesy services of doctors, nurses and other health care workers

- $\quad$ adequacy of infrastructure and environmental standards to meet end users' needs

- $\quad$ coordination and linkages should be strengthened between all role players for a comprehensive service delivery 


\subsubsection{Perceptions of end users concerning quality of antiretroviral counselling services}

Counselling about ART is thought to be important to prepare end users for treatment and enhance adherence. Stenson, Charalambous, Dwadwa, Pemba, Toit, Baggaley, Grant and Churchyard (2005:749) in a workplace-based HIV care programme in South Africa instituted a three step ART counselling protocol with guidelines prompting issues to be covered at each step. They carried out an early evaluation of ART counselling to determine whether end users understood key information about ART, and the perceptions that end users and health care professionals (HCP) had of the process. Among 40 end users (median time on ART 83 days), 95\% thought counselling sessions were good, specifying knowledge gained, increased hope and ability to make choices. Negative comments about counselling included problems with counsellors' attitude towards end users.

When asked who they preferred to talk to in clinic, 44\% said anyone, 33\% preferred doctors, $13 \%$ counsellors and $10 \%$ nurses. Up to $95 \%$ would recommend the health service to a friend or colleague and that another take ART. In a South African study, HIV-positive women confirmed the negative attitudes of health care workers who are biased against PLHIV having children (Nduna \& Farlane 2009:S62) with reports of forced contraceptive uptake in some instances (Nduna and Farlane 2009:S62).

In order to ensure continued quality care that meets end users' expectations, and for counselling to be effective, Stenson et al (2005:952) noted that end users must be empowered to recognise and accept quality of care. End users must feel able to communicate freely. Trust and willingness to participate are the foundation upon which the other objectives of counselling can be achieved. This could reflect a desire to report acceptable behaviour, but the high proportion who would recommend both the health service and taking ART to a friend or colleague suggests that participants had confidence in the ART programme and the treatment provided. In a study conducted in Uganda, Zambia and South Africa, to identify lessons learned for improving scale-up efficiency, Wagner, Ryan and Taylor (2007:871) lament that to minimise end user dropping out of care, structures (such as support groups) should be developed to enable end users provide support to each other, and have a "safety net" for helping each other remain in care. 
In another study conducted to explore the perception of rewards among volunteers working in home-based care settings, Akintola (2010:10) espoused that volunteer caregivers derived intrinsic rewards related to self-growth and personal (emotional and psychological) development on the job. They also derived satisfaction from community members taking a liking for them and expressing a need for their services (Akintola 2010:10). Akintola (2010:10) continued that volunteers felt gratified by the improvements in their health behaviours, which were direct consequences of the experiences of caring for terminally ill end users with AIDS. Extrinsic rewards came from appreciation and recognition shown by end users and community members (Akintola 2010:10). Akintola (2010:10) notes that extrinsic rewards also accrued to volunteers when the services they rendered made their end users happy. Perhaps the greatest sources of extrinsic rewards are skills and competencies acquired from training and experience while caring for their end users, and volunteers' ability to make a difference in the community (Akintola 2010:10).

In a study conducted in Egypt to determine the profile of end users and their level of satisfaction with health service, Kabbash, Hassan, Al-Nawasy, Attalla, and Mekheimer (2010:481) report that more than 90 percent of the end users were satisfied with counsellilng services in the health facility. In a randomised, prospective trial in an urban medical center to compare end user satisfaction with 3 different postpartum contraceptive counselling methods (health care worker-end user counselling, written literature or an educational video), Proctor, Jenkins, Loeb, Elliot and Ryan (2006:377) espoused that greater than 90 percent of end users in each arm were satisfied with contraceptive counselling that they received.

End users receiving health care worker-end user counselling expressed a 99 percent satisfaction rate (Proctor et al 2006:377). In this study, Proctor et al (2006:377) note that African American and Hispanic end users were more satisfied than Caucasian end users. Udoh et al (2011:223) reveal from a study in Nigeria that end users who had obstetric sonography in government health facilities were moderately dissatisfied with the protection of their privacy, whereas those who had obstetric sonography in missionary health facilities were moderately satisfied with the protection of their privacy. 
In conclusion, the literature of the perceptions of end users on quality indicated:

- $\quad$ end users cooperate (in support groups) with enthusiasm to improve the quality of life of peers

- $\quad$ health care workers empathise with end users in order to build confidence and enhance satisfaction/quality of life

\subsubsection{Perceptions of end users concerning quality of antiretroviral therapy pharmacy services in public health facilities}

Karunamoorthi, Rajalakshmi, Babu and Yohannes (2009:331) sought to determine end users' level of satisfaction and their expectations with pharmacy services at specialist ART unit in the government hospitals of Addis Abbaba, Ethiopia. Results of the study in Karunamoorthi et al (2009:337) showed that end users were dissatisfied with the pharmacy services. Major area of dissatisfaction was long waiting time in the pharmacy, which gulped $82,5 \%$ of respondents investigated. Some of them complained about lack of information on ARV drugs dispensed to them - most $(72,6 \%)$ wanted both written and oral information/instructions regarding the drugs provided. End users $(22,4 \%)$ also said they were dissatisfied because the pharmacists were impolite towards them.

In some cases, end users faced inadequate supplies of drugs, but could not tell what the cause of drug outages were. To review possible pharmacy related issues in support of the perceptions of end users, Alemayehu, Bushen and Muluneh (2009:356) assessed the quality of clinical care provided to end users with HIV in Felege Hiwot Referral Hospital, in North West Ethiopia. Alemayehu et al (2009:360) reported that out of the sample end users living with HIVIAIDS who were eligible for cotrimoxazole (CPT), only about $45,9 \%$ were actually on CPT. This considerably low achievement was because of failure to prescribe CPT for eligible end users during clinic visits, lower rate of continuity of care and poor compliance of end users with CPT.

Additionally, Alemayehu et al (2009:360) noted that the hospital studied was able to keep $76,8 \%$ of eligible end users on ART. Nearly $13 \%$ of the eligible end users were never started on ART and the remaining 10,5\% failed to follow-up after starting ART. This showed that there was more than a quarter $(28,8 \%)$ PLHIV who were eligible but yet to be put on ART. Similarly, continuity of care was maintained for $88,0 \%$ of end 
users in care after ART initiation and 37, 3\% before ART initiation. Only $41,9 \%$ of end users had a complete address documented in their records, making tracing of the end users difficult. Ramchandani, Mehta, Saple, Vaidya, Pandy, Vandrevu, Rajasekaran, Bhatia, Chowdhary, Bollinger and Gupta (2007:140) report lack of end users' confidence in utilising public health facilities, due to inadequate quality of ART services. Results of their study support previous reports that most newly diagnosed HIV persons enter the health care system via the private health sector. Less than 25 per cent of persons in their study were taking ART, and the vast majority of them (81\%) received their HIV care from private clinics. Surveys of private clinic attendees have found that persons prefer the private sector because there is more flexible and increased access, shorter waiting time, greater confidentiality, and greater sensitivity to user needs (Ramchandani et al 2007:140). However, poor quality of care has also been documented among private providers, particularly in the treatment of infectious diseases such as tuberculosis and malaria, where overuse and inappropriate prescribing has been demonstrated (Ramchandani et al 2007:140).

Barbosa (2011:103) conducted a study in the State of Rio de Janeiro, to describe the profile of users of the Palliative Care Unit of the National Cancer Institute and analyse the relationship between the profile and needs of its users with the pharmaceutical care provided. In this study, Barbosa (2011:103) reveal that satisfaction with pharmaceutical care received did not show variation in relation to their socioeconomic characteristics. However, when related to satisfaction with the fear of using drugs such as morphine, for example, there is fear that the smaller the higher the satisfaction. The results of this study show the need for education activities with users who mostly have low rates of education and further dialogue and clarification on the pharmacotherapy (Barbosa 2011:103). In another study in Vitoria, Brazil, to evaluate the satisfaction of users of a Pharmaceutical Care (PC) service in a private communitarian pharmacy, Andrade, Burini, Mello, Bersacula, Saliba, Bravim and Bissoli (2009:349) lament that a higher level of satisfaction was reported by users of the PC service, with values between three and five. The higher averages for the establishment with PC service were the result of greater perceived pharmacist interest in the end user's health. As the same results were not obtained by the services without PC, it was concluded that this practice is very important to the satisfaction level of users of pharmacy services. 
In conclusion, the literature of the perceptions of end users on quality indicated:

- $\quad$ uninterrupted, adequate supply and availability of ARV and other drugs for treatment and management of complications

- competent and adequate pharmacists should deliver quality of pharmacy services in ART clinics

- $\quad$ the supply chain management of laboratory reagents and drugs should be adequately available and useful for treatment

- $\quad$ a lifesaving ART programme is fundamental in public health services

\subsubsection{Perceptions of end users concerning quality of antiretroviral therapy laboratory services in public health facilities}

End users' satisfaction with laboratory services in public health facilities is important for the quality improvement of ART service provision. A study conducted in Tanzania, by Mfinanga, Kahwa, Kimaro, Kilale, Kivuyo, Senkoro, Ngowi, Mtandu, Mutayoba, Ngadaya and Mashoto (2008:1), sought to associate end users' satisfaction with the laboratory services. Mfinanga et al (2008:3) reported the study's findings which show that about $15,0 \%$ to $34,8 \%$ of end users were not satisfied with waiting time, privacy, results notification, cleanliness and timely instructions. End users who visited private health facilities were less dissatisfied with cleanliness and privacy of ART laboratory services than those who visited public health facilities. Also, end users with higher education were more likely to be dissatisfied with privacy and waiting time in both private and public health facilities.

Some end users tend to visit health facilities and return home without access to required laboratory tests. To provide a clue, Alemayehu et al (2009:356) assessed the quality of clinical care (including associated laboratory tests) for heatlh service users with HIV in Felege Hiwot Referral Hospital, North West Ethiopia. Results of the study in Alemayehu et al (2009:360) suggest that a relatively better quality of care was provided to end users on enrolment in care, with $90,9 \%$ of the end users received their first CD4 count within 2 weeks after first HIV clinic visit. For the remaining components of care expected to be provided as follow up care, the health facility failed to provide recommended care for significant portion of its HIV infected end users. Coulter (2010:2) report that end users could choose where they were referred to, or choose the specialist clinical team that will provide their treatment, if they so wish. For example, Coulter (2010:2) cited that 
when end users waiting for cardiac surgery were offered the choice of going to another health facility with a shorter waiting list, half of them opted to do so, sometimes traveling long distances. Similarly, a high proportion of end users in London awaiting various elective surgical procedures opted for alternatives to their local health facility when given the choice, and there was no evidence of socioeconomic differences in uptake (Coulter 2010:2).

In conclusion, the literature of the perceptions of end users on quality indicated:

- $\quad$ maintain linkages with relevant facilities to obtain specialised diagnostic services

- $\quad$ ensure uninterrupted laboratory services by reducing equipment down time and laboratory commodities stockout

\subsubsection{Perceptions of end users concerning antiretroviral pharmaceuticals based on material characteristics}

Schumaker and Bond's (2008:2130) study findings show that Zambians have expectations of pharmaceuticals based on material characteristics, associating some characteristics with familiar and effective drugs and wondering whether they are being given the correct drug when familiar characteristics change abruptly. Thus, new pharmaceuticals, such as ARVs, might arouse end users' concerns about efficacy (if in tablet rather than capsule form) and cause concern if there is a mismatch between their colours and expectations about links between colour and disease (for example, red for blood diseases). End users' attention to material characteristics assists intelligent decision-making about treatment in resource-poor settings as well as in wealthy end user societies. In Zambia, end users often move from clinic to clinic seeking better treatment, aware of drug shortages that mean they may not be prescribed the most appropriate or effective drug. To maximise impact on treatment outcome, social and economic health, it is important for ART programmes to develop effective mechanisms for coordinating and referring end users to other health facilities and support service organisations (Wagner et al 2007:871).

Overstretched health workers may not explain a drug's action in the body, leaving end users to rely on past experience of pharmaceuticals and their own traditional and biomedical knowledge to interpret side effects and efficacy after they begin treatment. 
Lessons learned from selected clinics in Uganda, Zambia and South Africa indicate that effective organisational management and human resource policies are essential to maintain high job performance and satisfaction and limit burnout (Wagner et al 2007:871).

Booysen, Van Rensburg, Bachmann, Louwagie and Fairall (2007:283) report from a treatment programme in Free State province of South Africa that statistical analysis of cross-sectional data reveals that it is not access to treatment per se that enhances the quality of life of those who have come forward for ART. Rather, it is the health benefits associated with treatment, levels of stigmatisation, quality health care services, and the ability of persons to access support and care, both from within and outside the health care sector, that are independently associated with improvements in and differences in levels of quality of life. Hopkins, Loeb and Fick (2009:927) concur that when end users come to a health facility, they expect to have a safe environment in which to receive ART, which will involve developing relationships with health care workers. They also expect their care to include one-to-one counselling, self-help groups, educational sessions, and information opportunities, communication with knowledgeable and empathetic staff and discharge planning.

In conclusion, the literature of the perceptions of end users on quality indicated:

- the health facility should be responsive to the needs of end users through leadership and policies

- $\quad$ end users emerge satisfied from the health facility

\subsubsection{Perceptions of end users concerning their interpersonal relationship with health care workers}

The nature of interpersonal relationship between health care workers and end users can be an important determinant of quality of ART service provision. Ding, Landon, Wilson, Hirschhorn, Marsden and Cleary (2008:35), in their study in the US, sought to compare the quality of care received by end users living with HIV who report that their primary HIV care provider is a physician, a nurse practitioner or a physician assistant to that of end users who cannot identify a primary HIV care provider. Findings of the study in Ding et al (2008:40) indicate that about $96 \%$ of end users in this sample (representing about 
217,000 end users nationally) said that a physician, a nurse practitioner or a physician's assistant knew them the best and took responsibility for their overall HIV medical care, but about $4 \%$ of the end users did not identify a primary HIV care provider. This is a small percent of all end users in care nationally who are often economically disadvantaged and more likely than other end users to have a history of injection drug use. Even after adjusting for end users and clinic characteristics, those end users were less likely than others to receive ART and were less satisfied with their care (Ding et al 2008:40). Ding et al (2008:40) elaborate that care can be poor even at sites with good support services if there is not a suitable health care worker relationship. The effective use of medical and support services probably requires a health care worker who knows each end user and can facilitate the provision and integration of care (Ding et al 2008:40). To evaluate the users' satisfaction with ophthalamologic service provided in South of Brazil, Benazzi et al (2010:861) note that 75,4 percent pointed deficiencies related to the interpersonal relationship with the health care workers. Madeiros et al (2010:402) affirm that a relationship involving respect and health care workers really listening to their end users makes the difference in health provision.

However, the compaints made must also be considered for effective embracement of the service being provided in the health facility (Madeiros et al 2010:402). Wouters et al (2008:210) reported that findings from a study conducted in South Africa showed that because human resource shortages are associated with lower levels of end user satisfaction, the quality of care is definitely affected by heavy workloads. Human resource shortages, combined with large numbers of end users in need of treatment, caused inadequate relationship building and further extended waiting times. These in turn were the most important source of discontent among PLHIV attending the ART assessment sites studied in the Free State. This could seriously hinder any successful ART scale-up, because several previous studies have shown that both waiting times and low end user satisfaction levels with ART related services hinder ART adherence (Wouters et al 2008:210).

In conclusion, the literature of the perceptions of end users on quality indicated:

- $\quad$ health care workers or support group volunteers should strive to treat end users with courtesy and affability, to improve their quality of life 
- $\quad$ health facilities should promote opportunities for interaction of colleagues and end users during service delivery

- $\quad$ health facilities should strengthen systems for end user complaints management

\subsubsection{Perceptions of end users concerning respect for their choices/preferences for antiretroviral therapy services}

Miller, Huffman, Weidmer and Hays (2002:593) investigated end user preferences regarding ART, in Torrance, California. Miller et al (2002:598) report in their findings that end users were willing to compromise items that may impact quality of life (i.e., increased side-effects, inconvenience, and pill burden) to have a potent antiretroviral regimen. End users also preferred regimens with fewer side-effects to those with less inconviniences. Pill burden was found to be of relatively low importance for end users.

Schumaker and Bond (2008:2130) in their study to explore the meaning of antiretrovirals in Zambia, elucidate that end users saw the side effects of ARVs as evidence of their strength and compared these to the side effects of septrin, TB drugs and fansidar (the latter until recently an effective malaria medication commonly used in Zambia). Indeed, ARVs, followed by TB drugs, were considered to have the most side effects, ahead of medications for malaria, cancer, diabetes, epilepsy, herpes zoster and sexually transmitted infections (STIs).

The most common ARV side effects reported were initial vomiting and diarrhoea, followed by increase in appetite (referred to as 'hunger'), weight gain (especially around the stomach), rashes, numbness and a change in the shape of the face ('swollen lips', 'big cheeks'). These side effects were shared with TB treatment but ARVs were also associated with more positive side effects, such as renewed strength. One informant in Schumaker and Bond's (2008:2131) study complained about the redistribution of weight, which looked like the early stages of pregnancy. Lipodystrophy - redistribution of fat - is a common side effect of the ARV regimen used in Zambia. This, along with general weight gain, can lead to stigma particularly for women (Schumaker \& Bond 2008:2131). The hunger linked to ARVs is especially poignant in a country where $73 \%$ are classified as poor (Schumaker \& Bond 2008:2130). Hunger has long been associated with TB medication - some in this study called it the 'No. 1' side effect. A 
limited number of TB end users in Zambia receive food aid during treatment (Schumaker \& Bond 2008:2131). The hunger associated with ARVs is now an emerging concern of HIV-advocacy groups in Africa (Schumaker \& Bond 2008:2131).

Ananworanich, Pumpradit, Apateerapong, Siangphoe, Saenawat, Phanuphak and Hirschel (2008:327) evaluated end users preferences between CD4-guided highly active antiretroviral therapy (HAART) and continuous HAART, in a structured treatment interruption (STI) trial in Thailand. Ananworanich et al (2008:329) found that more end users preferred continuous HAART. The main reasons for not choosing CD4-guided HAART were fear of developing HIV-related illnesses and fear of resistance. Ananworanich et al (2008:329) further found that having a higher CD4 nadir (the lowest CD4 count one have ever had), fewer on-off cycles and a slightly less CD4 drop from baseline contributed to end users choosing CD4-guided HAART. This is likely due to their relatively higher CD4 count resulting in less fear of a negative clinical outcome.

In conclusion, the literature of the perceptions of end users on quality indicated:

- leadership is needed to implement innovative approaches to improve dialogue with health authorities to improve quality of life of people on ART

- $\quad$ ensure availability and provide opportunities for end users to choose from available lifesaving antiretroviral drugs

\subsubsection{Perceptions of end users concerning timeliness of health services}

According to the United States Agency for Health Care Research and Quality - AHRQ (2010:147), timeliness is the health care system's capacity to provide care quickly after a need is recognised. It is one of the six dimensions of quality the Institute of Medicine established as a priority for improvement in the health care system. Measures of timeliness include time spent waiting in doctor's offices and emergency departments and the interval between identifying a need for specific tests and treatments and actually receiving services. Arising from quality improvement in the US health care services, the AHRQ (2010:148) reports that from 2002 to 2007, the overall percentage of adults who needed care right away for an illness, injury, or condition in the last 12 months who sometimes or never got care as soon as wanted decreased (from $16.8 \%$ to $15.3 \%)$. 
The AHRQ (2010:147) itemised the following as benefits of timeliness to the health care system:

- Lack of timeliness can result in emotional distress, physical harm, and higher treatment costs for end users.

- $\quad$ Timely delivery of appropriate care can help reduce mortality and morbidity for chronic conditions, such as AIDS.

- $\quad$ Timely antibiotic treatments are associated with improved clinical outcomes.

- $\quad$ Early care for co-morbid conditions (such as TB and HIV) has been shown to reduce hospitalisation rates and costs for medical care.

Wouters, Heunis, Rensburg and Meulemans (2008:1) conducted a study to determine end user satisfaction with ART services at primary health care facilities in the Free State, South Africa. Although there was high overall satisfaction among Free State end users receiving public sector ART, end users in Fezile Dabi District were generally slightly dissatisfied with the waiting times at their assessment sites.

In fact, Wouters et al (2008:11) report that overall, dissatisfaction with waiting times at assessment sites seems to have been the most important predictor of discontent among end users receiving ART. Findings showed that, because human resource shortages are associated with lower levels of end user satisfaction, the quality of care is definitely affected by heavy workloads (Wouters et al 2008:11). Wouters et al (2008:11) add that human resource shortages, combined with large numbers of end users in need of treatment, caused further extended waiting times, which were the most important source of discontent among the PLHIV. This could seriously hinder any successful ART scale up, because several previous studies have shown that both waiting times and low end user satisfaction levels with ART-related services hinder ART adherence (Wouters et al 2008:11).

High satisfaction ratings have been reported among end users in fewer health facilities around the world. In a study by Oliveira, Arieta, Temporini and Kara-Jose (2006:731), to evaluate end user characteristics and satisfaction in an outpatient service at the Hospital das Clinicas, Universidade Estadual de Campinas, Brazil, the majority of respondents reported that it is easy to obtain appointmets, the mean waiting time for 
appointment was 94,6 minutes and end users were satisfied with timeliness in this health facility. It is important to note that the sample was characterised by end users with low literacy and socioeconomic level (Oliveira et al 2006:731). This contrasts with a similar study by Aldana, Piechulek, Alsabir (2001:512) which assessed end user expectation and degree of satisfaction and quality of health care provided in rural Bangladesh. Findings of this study revealed that a reduction of waiting time (on average to 30 minutes) was more important to end users than a prolongation of the quite short short (from medical standpoint) consultation time (on average 2 minutes, 22 seconds), with 75 percent of end users being satisfied (Aldana et al 2001:512). To evaluate the satisfaction of end users with health services provided in northwest Parana State, Muller and Greco (2010:925) report that the end users were happy with the time taken to set appointments and the time taken for consultations in the health facility.

Blumenthal, Gaffikin, Deganus, Lewis, Emerson and Adadevoh (2007:407e1) conducted a study in Ghana, to assess the safety and acceptability of a single-visit approach to cervical cancer prevention combining visual inspection of the cervix with acetic acid wash (VIA) and cryotherapy. Findings of the study reveal that at the first follow-up visit, almost all women (99.4\%) indicated being either very satisfied or satisfied with their decision to have the condition of their cervix assessed using VIA. Most also indicated that they were either very satisfied (87.2\%) or satisfied (12.5\%) about their decision to be treated with cryotherapy for suspect cervical disease. Most (98.6\%) had already recommended to friends and/or family members that they get VIA tested, and all respondents indicated that they would do so in the future (Blumenthal et al 2007:407e4).

Timeliness is a challenging end user requirement to attain in public health facilities, where long waiting times have negatively affected satisfaction ratings (Chow et al 2009:438; Rad, Haghani, Shahravan \& Khosravifar 2009:377; Chavez de Paz, Ramos \& Galarza 2009:22). In Brazil, a study conducted in Pernambuco State, by Gouvela, Souza, Carlos, Szwarcwald, Souza Juniour and Roberto (2011:1849) reveal that the waiting time for health care and the availability of medicines were the factors with the lowest percentage of users' satisfaction. To evaluate the users' satisfaction with ophthalamologic service provided by an accredited service of the Brazillian Unified Health System in the urban center of the South of Brazil, Benazzi et al (2010:861) reveal that 75.4 percent pointed deficiencies related to the time they had to wait in the 
place to the duration of the consultation. Udoh et al (2011:222) show that obstetric sonography end users were moderately dissatisfied with the waiting time in both public and and missionary hospitals.

In a study conducted in the central pharmacy of Hospital Nacional Dos de Mayo, in Lima, to determine the influence of waiting time on end user's satisfaction, Gutierreze, Ramos, Uribe, Ortega-Loayza, Torres, Momntesinos, Leon and Galarza (2009:61) reveal that the waiting time was significantly higher in the sub billing process and also the time that users took long queues was higher than the actual time. Only 17.3 percent of the users of the central pharmacy showed some degree of satisfaction. Muza and Muza (2008:12) conducted a study to assess the degree of satisfaction of female end users with three dental specialties: dontopediatrics, periodontics and oral surgery. In their findings, the areas that resulted as less than average after assessment, included the obtainment of appointments in a prescheduled time, and the waiting time at the clinic to gain access to the dentist (Muza \& Muza 2008:12).

In another study conducted in Porto Alegre, Brazil, to describe end users' opinions regarding access and quality care in health facilities, Lima, Ramos, Rosa, Nauderer and Davis (2007:12) reveal that users reported they were subjected to long waiting periods; they had to arrive very early to be seen. Gaioso and Mishima (2007:1) conducted a study to assess users' acceptability of the care offered and delivered by Family Health teams in Ribeirao Preto, Sao Paulo, Brazil. Findings of the study indicated a long time spent in the waiting room. In a study to analyse satisfaction with health care based on the results of the World Health Survey, conducted in Brazil in 2003, waiting time showed the lowest degree of satisfaction, especially, among end users who were treated as outpatients.

Process improvements have proven helpful in reducing waiting time in health facilities. For example, Wong and Bradley (2009:253) sought to evaluate the impact of an inexpensive business process re-engineering project on the accessibility and completeness of end user information and satisfaction in Ethiopia. Findings reveal that medical record accessibility and completeness, and satisfaction improved significantly $(p<0.05)$ based on pre- and post-intervention comparisons. The success rate of retrieving the proper medical record number for returning end users improved from 14 to 
87 percent $(p<0.01)$; time to locate medical records decreased from 31.2 seconds per record to 15.7 seconds per record $(p<0.01)$.

Thus, depicting that a well-organised medical record management system can be effective in reducing waiting time and improving end user information accessibility and completeness in health facilities in low-income countries, despite the lack of resources (Wong \& Bradley 2009:253). Lessons learned from projects in Uganda, Zambia, and South Africa also provide that processes can be improved, and end user waiting time reduced. For example, Wagner et al (2007:871) reveal that minimising bottlenecks to smooth end user flow requires efficient staff allocation to appropriate clinical duties, streamlined clinic visit schedule protocols, and tapping end users and the HIV community as a key source of labour.

In conclusion, the literature of the perceptions of end users on quality indicated:

- $\quad$ inadequate treatment of end users could be related to inadequate resources

- $\quad$ standardisation of health care processes and procedures is needed to promote workflow

- $\quad$ standardise processes and procedures to reduce end user waiting time seeking health care

\subsubsection{Perceptions of end users concerning end user centeredness}

The AHRQ (2010:153) identified end user centeredness as a core component of quality health care. End user centeredness is defined as health care that establishes a partnership among practitioners, end users, and their families (when appropriate) to ensure that decisions respect end users' wants, needs, and preferences and that end users have the education and support they need to make decisions and participate in their own care. Coulter (2010:4) views shared decision-making as a process in which end users are encouraged to participate in selecting appropriate treatment or management options. Shared decision-making is appropriate whenever there is more than one reasonable course of action and not single option is evidently best for everyone. This situation is common because there are often different ways to treat a health problem, each of which may lead to different outcomes. These are known as 
"preference sensitive" decisions, and the end users' attitude to the likely benefits and risks must be a key factor in the decision (Coulter 2010:4).

Coulter (2005:1200) hypothesise that knowing about the various treatment options available and having a say in these is more important to most end users than having a choice of where to be treated. Coulter (2005:1200) reveals that most end users value the opportunity to choose their health care workers. There is almost certainly scope to extend the choices available, but the heaviest users of primary care (older people and those with chronic conditions) place particular value on continuity of care from health care workers they know (Coulter 2005:1200).

To maximise impact on social and economic health therefore, Wagner (2007:871) recommends that it is important for ART programmes to develop effective mechanisms for coordinating and referring end users to support service organisations. This recommendation is supported by Colvin et al (2003:S86) who report that interviews with 41 of 51 traditional healer end users who had completed tuberculosis treatment in Hlabisa, KwaZulu-Natal, South Africa, revealed high levels of satisfaction with the care received. Recent evidence confirms that despite many health care workers' ambivalence or antagonism to the idea, most end users are keen on having a choice, or the right to make decisions, even if they choose to remain at their local health facility (Coulter 2010:3). Coulter (2010:3) added that, despite a widespread view among health care workers and hospital managers that the right to end user choice is relevant only in urban centres, a King's Fund study found that end users living in rural areas were both more likely to be offered a choice and more likely to travel to a non-local provider.

The AHRQ (2010:153) elucidates that end user centeredness encompasses qualities of compassion, empathy, and responsiveness to the needs, values, and expressed preferences of the individual end user. End user-centred care is supported by good provider-end user communication so that end users' needs and wants are understood and addressed and end users understand and participate in their own care. This approach to care has been shown to improve end users' health and health care. Following quality improvement initiatives in the US, AHRQ (2010:156) notes that from 2002 to 2007, the percentage of adults with a doctor's office or clinic visit who reported poor communication significantly decreased, from $10.8 \%$ to $9.3 \%$. In all years, adults with basic or complex activity limitations were more likely to report poor communication 
than adults with neither basic nor complex activity limitations. It is therefore, important that both end users and providers not assume that instructions are understood but develop a means to show comprehension.

Coulter (2005:1199) elucidate that end users want an explanation of their symptoms, treatment, or investigation. Many have their own ideas about what is wrong and what may have caused it, but they do not always articulate these. For example, in a study conducted in three Russian cities to explore end user satisfaction with abortion care, looking at overall satisfaction and satisfaction with hygiene, comfort and courtesy, Olivera, Larsen and David (2005:585) reveal that information provided to abortion end users about self-care was the most important predictor of overall satisfaction for the abortion end users. Failure to engage with the end user's agenda can lead to misunderstandings, dissatisfaction, and poor outcomes (Coulter 2005:1200). Coulter (2005:1201) espouse that evidence is growing that engaging end users in treatment decisions and managing their health care can lead to more appropriate and cost effective use of health services and better health outcomes.

Although including end users in planning developments to the ART service has had some positive results, Coulter (2005:1201) warns that many of the current initiatives that go under the banner of end user or public involvement are little more than window dressing. Including a few token end users (such as network of PLHIV) on committees is relatively easy, but it does little to tackle the heart of the problem (Coulter 2005:1201). Agreeing with this point, Banks and Halasa (2005:1), in their Jordan study, to assess end users' opinion on level of communication (among other variables), reported that health care workers fail to communicate with end users about medical issues such as diagnosis, treatment options, length of hospital stay, and length of recovery. In the same study, 13 percent of end users were reported not to have been given physical examination, upon admission (Banks \& Halasa 2005:1).

Reporting on health care worker-end user communication in a study by Udo et al (2011:223), end users revealed that they were moderately dissatisfied with the level of communication they received from health care workers in public health facilities, but were moderately satisfied with the communication from private health facilities. This finding agrees with that of Coulter (2010:4) which revealed that not being properly informed about their illness and the options for treatment are the most common causes 
of end user dissatisfaction. In other words, Coulter (2005:1201) elucidates that failures in communication of information about illness and treatment is the most frequent source of end user dissatisfaction.

In order to communicate factually and appropriately with the end user, precise examination of the end user's health problems and comprehensive treatment plans must be developed (Rad et al 2009:377). For example, in a study conducted to describe mother's satisfaction with perinatal care received during hospitalisation for delivery, and to identify sociodemographic and health care related factors associated with satisfaction, Senarath, Fernando and Rodrigo (2006:1442) report that the determination of satisfaction included providing immediate mother-newborn contact, information after examination and counselling on family planning.

AHRQ (2010:154) itemised the following as benefits of end user centeredness to the health care system:

- $\quad$ End user-centered approaches to care have been shown to improve end users' health status. These approaches rely on building a provider-end user relationship, improving communication, fostering a positive atmosphere, and encouraging end users to actively participate in provider-end user interactions.

- $\quad$ End user-centered approach encourages end users to comply with treatment guidelines.

- $\quad$ End user-centered care can reduce the chance of misdiagnosis due to poor communication.

- $\quad$ End user centeredness has been shown to reduce underuse and overuse of medical care.

- $\quad$ End user centeredness can reduce the strain on system resources and save money by reducing the number of diagnostic tests and referrals.

- $\quad$ Although some studies have shown that being end user centered reduces medical costs and use of health service resources, others have shown that end user centeredness increases providers' costs, especially in the short run. 
In conclusion, the literature of the perceptions of end users on quality indicated:

- health facilities should ensure that any end user who turns up to the health facility benefit from full assessment of his/her current health status; and is counselled if needed

- $\quad$ strengthen linkages between support groups and community based organisations to promote accessibility and availability of wrap around services

- $\quad$ recognise the essence of capacity building and motivational programmes to meet task requirement

- $\quad$ incorporate HIVIAIDS into regular health services, and encourage end users to attend regular clinics

\subsubsection{Perceptions of end users concerning end user safety}

The US National Priorities and Partnership identified safety in health care as one of six national priorities. The goal is a health care system that is: ... relentless in continually reducing the risks of injury from care, aiming for "zero" harm wherever and whenever possible - a system that can promise absolutely reliable care, guaranteeing that every end user, every time, receives the benefits of care based solidly on science (AHRQ 2010:135). AHRQ (2010:135) elucidates that the goal of end user safety include:

- $\quad$ Health care facility and staff ensuring a culture of safety in order to reduce health care-associated infections (HAIs) and serious adverse events (SAEs).

- $\quad$ Health facilities reducing preventable and premature health facility level mortality rates.

- $\quad$ Health facilities improving their 30-day mortality rates of selected conditions (e.g., pneumonia).

Following quality improvement initiatives in the US, AHRQ (2010:138) reports that the percentage of adult surgery end users who received appropriate timing of antibiotics improved from 2005 to 2008 (74.9\% to 91.4\%). Improvements were also seen among all age groups during this period. Also, from 2004 to 2007, the rate of deaths following complications of care declined from 128.9 to 105.7 per 1,000 admissions of adults ages 18-74. A significant decrease was also seen among all geographic, gender, and 
insurance groups during the same period (AHRQ 2010:143). Finally, AHRQ (2010:145) notes that while national 30-day mortality rates due to pneumonia are not currently available for reporting; the in-health facility mortality rates per 1,000 health facility admissions with pneumonia are reported. About two-thirds of end users who die within 30 days of admission die inside the health facility, and the correlation between in-end user and 30-day mortality is high (AHRQ 2010:144). From 2004 to 2007, the in-end user pneumonia mortality rate decreased overall from 55.2 per 1,000 admissions to 40.8 . During the same period, a significant decrease was also seen among all geographic areas and among males and females (AHRQ 2010:145).

In a study to understand the meaning of quality of life for PLHIV, conducted in four subSaharan Africa countries of Botswana, Lesotho, South Africa and Swaziland, the participants who expressed higher satisfaction scores were those who were less educated, had worries about disclosure and finances, were not on ART and had fewer health worries (Phaladze, Human, Dlamini, Hulela, Hadebe, Sukati, Makoae, Seboni, Moleko \& Holzemer 2005:120). This may suggest that those who were on ART and were better educated could have presented with a better judgment of their perceptions of quality of care. Joolaee, Hajibabaee, Jafar Jalal and Bahrani (2011:1), in their study to assess end users' satisfaction from nursing care in hospitals of Iran University of Medical Sciences in 2010, agree that level of education was significantly associated with end user satisfaction. However, fear about disclosure of HIV status is a recurring issue in most studies. For example a South African study by Ginwalla, Grant, Day, Dlova, Macintyre, Baggaley and Churchyard (2002:707) reveal that end users raised concerns about confidentiality of their HIV status, and indeed, their health care in general. Another South African study by Myburgh, Solanki, Smith and Lalloo (2005:473) was set out to assess the influence of race and socioeconomic status on perceived quality of care.

Findings of this study reveal that both race and socioeconomic status were significant predictors of level of satisfaction with the services of the health care workers, after adjusting for gender, age, and type of facility visited (Myburgh et al 2005:473). Myburgh et al (2005:473) elucidate that White and high socioeconomic respondents were about 1.5 times more likely to report excellent services compared with Black and low socioeconomic respondents, respectively. This brings to bear the need for respect of end users and to ensure none disclosure of end user information to unauthorised 
persons, if end user satisfaction is to be attained. In a study to relate sociodemographic variables, anxiety levels and oral health beliefs with the level of end user satisfaction in oral health (a common problem among PLHIV), Lopez Soto, Cerezo Correa and PazDelgado (2010:124) report that dissatisfied end users were characterised by low selfesteem, fears of visiting a health care worker and sociodemographic characteristics, a pointer to the need to addressing stigma and discrimination against all end users. Coulter (2005:1200) canvases for a well coordinated and better community support and continuity of care, to ensure end user safety, rather than a diversity of competing providers who have no clue on infection control. Coulter (2006:1200) also supports the urgent need to impove support for self care and self management, to reduce the number of exposures end users will have with an infectious environment in a health facility, while building end user confidence and reducing stigma. Liu and Wang (2007:266) re-iterate that end users who are satisfied with health care have a greater sense of safety during their entire stay in the health facility. For example, Udo et al (2011:223) report that end users were moderately satisfied with the level of safety they experienced in both public and private health facilities in Nigeria.

In conclusion, the literature of the perceptions of end users on quality indicated:

- $\quad$ infection prevention and control should be a priority in a facility

- $\quad$ a stigma free environment for users should be ensured

\subsubsection{Perceptions of end users concerning quality of communication and information shared with them}

One of the most widespread complaints from end users, in general, is that of not receiving sufficient information about their illness and treatment, or the information that is provided is often too complex to understand and retain (Gellaitry, Cooper, Davis, Fisher, Leake, Date \& Home 2005:367). For example, Pietrovsk and Dall'Agnol (2006:630) posit from a study among end users on haemodialytic treatment, that difficulties were associated with communication process between health care workers and the end users. Similarly, in a study conducted in Iran to clarify the perceptions of end users on the quality of family planning services, Nakhaee and Mirahmadizadeh (2005:192) espouse that the provision of insufficient information regarding other contraceptive methods caused dissatisfactions among the end users. 
In a Brighton, UK study, Gellaitry et al (2005:374) profiled end users' satisfaction with information they have received about HAART in relation to treatment uptake. Results of the study indicated that those who accepted the offer of HAART were more satisfied with the information they had received about treatment than those who declined the treatment offer. Although causality cannot be inferred, this finding clearly shows that those end users who decline or defer treatment are less likely to report that the information they have received about their recommended therapy has met their individual needs. Those who held stronger concerns about treatment were less satisfied with the information they had received (Gellaitry et al 2005:374). Similarly, Oliveras, Larsen and David (2005: 585) report from a Russian study that information provided to abortion end users about self-care was the most important predictor of overall satisfaction for the end users. This drives home the fact that health care worker - end user relationship factor could serve as a barrier to end user satisfaction and ART adherence (Rao, Kekwaletswe, Hoser, Martinez \& Rodriguez 2007:28).

Gellaitry et al (2005:374) indicated that end users who are dissatisfied with the information they have been given are less likely to follow advice or adhere to their prescribed treatment. For example, it stemmed from a study conducted in Nigeria by Ehlers and Chiegil (2011:6) that end users who have inadequate knowledge of ART often fail to adhere to their prescribed ART regimen. Knowing end users' preferences for information can help to guide health care workers in their consultations with end users, enabling them to focus on the specific needs of the individual end user rather than simply disseminating more and more information about prescribed drugs.

The best way of judging the quality of information provided to end users is to assess whether they perceive it as having met their individual needs (Gellaitry et al 2005:374). In a study at a University Research and Extension Center in Ribeirao Preto, to describe the satisfaction of end users with diabetes, using information received and the care offered after the implementation of the health educational programme in April 2005, Zanetti, Otero, Biaggi, Santos, Peress and Guimaraes (2007:583) unveil that end users reported that the information received during the programme met their needs.

Related to the assessment of care, 81.5 percent of the end users considered it excellent (Zanetti et al 2007:583). The level of end user satisfaction was investigated in regular 
attendees at public health chronic disease facilities in South Trinidad (Joseph \& Nichols 2007:108). Joseph and Nichols (2007:108) posit that approximately two-thirds of participants gave health care workers a rating of good to excellent. Fifty-three and a half percent and 58 percent gave a poor to fair rating for the length of the waiting time and explanation offered when there was a significant delay in the starting times of clinics, respectively. In a separate study conducted in South Brazil by Sisson, Olivera, Conill, Pires, Boing and Fertonani (2011:123), user satisfaction showed favourable results where there was fast access to appropriate technical resources, while dissatisfactions were relevant to difficulties in information availability, costs, among others. It is therefore, best practice that the interdisciplinary health care team be involved in adequate information sharing, especially, during end user discharge home. For example, Pompeo, Pinto, Cesarino, Araujo and Poletti (2007:345) reveal that among a majority of end users who received trailored discharge instructions from a health facility in Brazil, almost half reported that the discharge instructions were given by physicians alone. A great number of end users (72.08\%) reported that discharge instructions were not given by nurses, and this caused dissatisfaction in the end users (Pompeo et al 2007:345).

In conclusion, the literature of the perceptions of end users on quality indicated

- $\quad$ knowledge and information improves relationships and improve quality of care

- $\quad$ strengthen systems to promote dialogue between support group members and health facility leadership

- $\quad$ strengthen systems to promote dialogue within the support group membership

\subsubsection{Perceptions of end users concerning their rights to decision-making regarding their care}

Gellaitry et al (2005:374) contained that whilst the decision to initiate HAART is clearly with clinical variables (a lower CD4 count and shorter length of time since HIV diagnosis), end users' concerns about potential adverse effects of treatment and satisfaction with the information they have received about HAART play a significant role in the treatment decision-making process, independently contributing to the variance in HAART uptake. It was, however, not surprising to Gellaitry et al (2005:374) that end 
users on the whole looked to specialised HIV professionals for information about HAART at the time of a recommended treatment offer.

End users found the face-to-face consultation with the health care workers to be most helpful sources of information at this point. This hightlights the importance of two-way interactions between the health care worker and end user, in order to achieve optimal levels of end user satisfaction with information about their treatment. Finally, Gellaitry et al (2005:375) recapped that the results of this study indicate that HIV-positive individuals differ widely in their information requirement when faced with making decisions about their health. The decision to accept a recommended offer of HAART is associated with greater levels of satisfaction with the information received about HAART and lower concerns about potential adverse effects of treatment. In a study conducted in the US, Beach, Duggan and Moore (2007:1119) sought to compare health care processes and outcomes of end users with HIV based on their preferred level of involvement in health decisions. Results of the study indicate that end users who preferred to share decisions with their health care worker had better outcomes than those who wanted their health care worker to make decisions and those who wanted to make decisions alone (Beach et al 2007:1122).

In addition, Beach et al (2007:1123) reported that end users who preferred to make decisions alone were less likely to be on HAART when it was clinically indicated. End users who preferred to share decisions with their health care worker reported better communication with their health care worker (Beach et al 2007:1123). This suggests that health care workers may be able to influence the decision-making preferences of their end users through good communication. In addition, Beach et al (2007:1123) found that end users who wanted to share decisions were also more likely to feel they were known "as a person" by their health care worker than end users who wanted to make all decisions alone. This further suggests that one way for health care workers to assist highly independent end users in gaining trust and accepting advice might be to build a more personal relationship with them. Coulter 2005:1200) supports this assertion by revealing that end users with chronic conditions place particular value on continuity of care from health care workers they know and have built relationships with. In an assessment of care from the end users' perspective, Silva, Beck, Lopes, Magnago, Prestes and Tavares (2010:1) reveal that end users feel more satisfied when they feel they are trusted and that they too can trust the health care workers. 
The end users visualise as less satisfactory the skills of the health care workers in providing information for follow up (Silva et al 2010:1). In a separate study by Chavez de Paz et al (2009:22) to determine end users' satisfaction in the dermatology service of the Dos de Mayo National Hospital, there was higher end user satisfaction with health care services that were associated with good information provided by health care workers about their disease. Santos et al (2008:151) also espoused from their study conducted among end users with chronic kidney disease in Minas Gerais, that the great majority (95,6 percent) of the end users reported to be satisfied with the explanation given about their disease. In a study conducted in Chile, to assess the degree of satisfaction of female end users with dental services, Muza and Muza (2008:12) highlights that the participants were in average satisfied with the explanations the dentist gave to them concerning the dental treatment and the access to the dental treatment. Better community support for health care workers and for end users on chronic illnesses are also perennial themes in end user surveys (Coulter 2005:1200). These groups require well cooordinated local services rather than a diversity of competing health care workers or health care facilities (Coulter 2005:1200).

Studies have found that end users who report greater involvement in medical care are more satisfied with their health care workers (Beach et al 2007:1119). They also report more understanding, reassurance, and perceived control over their illness, and have improvements in medical conditions. For example, in an Iranian study of end user satisfaction, Joolaee et al (2011:1) report that end users were moderately satisfied from nursing care services which ensured end user participation in decision-making. A similar finding was reported by Udoh et al (2011:224), where end users were moderately satisfied with services rendered to them during obstetric sonography in private health facilities in Nigeria. In this study, moderate dissatisfaction was reported from public health facilities in Nigeria (Udoh et al 2011:224). Another study which highlighted end user satisfaction arising from participation of end users in decisionmaking regarding their care was documented in Liu and Wang (2007:269).

Morin, Godin, Alary, Sawadogo, Bernier, Khonde, Kintin, Kone, N'Dour, Pepin, Rached, Sobela, Soto, Sylla and Traore (2008:388) conducted a study to assess the degree of satisfaction regarding HIV and sexually transmitted infections services for women rarely listened to in, associated with, or living in or nearby, prostitution environments in seven 
West-African countries (Benin, Burkina Faso, Ghana, Mali, Niger, Senegal, and Togo). Regression analysis showed that those most satisfied were women who had used the adapted services many times and women connected with community groups (Morin et al 2008:388). To measure the extent of quality differentials in family planning (FP) services at public and private health facilities and clinics in three countries (Ghana, Kenya, and Tanzania), Hutchinson et al (2009:1) report that private health facilities appear to be of higher process but not necessarily technical quality in the three countries measured by several quality attributes.

End users' satisfaction, however, appears considerably higher at private health facilities, most likely attributeable to other factors such as shorter waiting times and fewer stockouts of methods and supplies, rather than only end user participation in the care process. It is unlikely that one factor alone will determine end user satisfaction with health care services in a given health facility. Therefore, several factors might have played key roles in the findings reported by Hutchinson et al (2009:1). For example, Booysen et al (2007:283) reports on the quality of life of end users enrolled in the public sector ART programme in the Free State province of South Africa. Statistical analysis of cross-sectional data reveals that it is not access to treatment per se that enhances the quality of life of those who have come forward for ART.

Rather, it is the health benefits associated with treatment, levels of stigmatisation, quality health care services, and the ability of persons to access support and care, both from within and outside the health care facility, which contributes to end user satisfaction. Another study by Ricci, Wanderley, Oliveira and Rebellato (2011:1125) assessed the satisfaction of the users of Sao Carlos Hospital School in its first six months of functioning. Findings of the study showed that the adoption of a humanised and user focused model of assistance in health care in this hospital resulted in end user satisfaction (Ricci et al 2011:1125). Similarly, care appeared highly valued by end users in eight community mental health care centres evaluated by Priebe, Matanov, Demi, Simic, Jovanovic, Gajic, Radonic, Bajraktarov, Boderscova, Konatar, Nica, Muijen (2011:1) in Albania, Bosnia-Herzegovina, Croatia, Macedonia, Moldova, Montenegro and Romania. Also, Machado, Medina and Kara-Jose (2010:276) apprised from a study to evaluate the assistance quality in Ophthalmologic Clinic of Divinolandia, through the perception of the end users that medical assistance was considered excellent by $79.6 \%$ 
of end users. Machado et al (2010:276) posit that reception received a great evaluation by $73.8 \%$ of the end users.

Another study was aimed at evaluating end user satisfaction with dental care at four specialised dental clinics in Recife, Pernambuco State, Brazil (Lima, Cabral \& Vasconcelos 2010:991). Findings of this study indicated that most users were satisfied with the dental care, and satisfaction differed between services (Lima et al 2010:991). To assess user satisfaction degree in an EsSalud primary health care centre (Peru), Cuba-Fuentes, Jurado and Estrella (2011:4) reveal that the users were satisfied with geographical accessibility, infrastructure, hours of care, problem-solving and diagnostic tests carried out in the centre. In another study to determine the quality of care provided by the health care team that worked in the preventorium of Callao in the period August to December 2009, end users report that the quality of nursing care rendered was mostly low to medium (Gallardo-Ramirez 2010:60).

Castillejo Nieves (2010:73) conducted a study to determine the level of external end users' satisfaction on the quality of nursing care at the Hospital Surgery Center Support in o'Victor Ramos Guardia-Huaraz, in 2009. Findings of the study reveals users' opinion on the satisfaction of care of the nurse in the human dimension of 100 percent (30): 47 percent (14) had a low satisfaction, 43 percent (13) average and 10 percent (3) high. Low satisfaction was scored when the user reports that the nurse was not identified as a professional who will attend to them, indifferent to their fears and concerns, indifferent to the call for action, or has lack of courtesy (Castillejo Nieves 2010:73). Miranda and Vargas (2009:1) also conducted a study in Brazil, to ascertain end user satisfaction with nursing care from a psychosocial care center for alcohol and drugs.

Findings of the study showed that most end users were satisfied with care provided by nurses, evaluating the care received as very good and showing trust in this professional to report their personal problems (Miranda \& Vargas 2009:1). In a separate study by Agha, Karim, Balal and Solsler (2007:320), to evaluate the impact of a nurse and paramedic reproductive heatlh franchise in rural Nepal on end user satisfaction and utilisation of services. Findings of the study by Agha et al (2007:320) revealed that end user satisfaction with the quality of services increased across a range of indicators at intervention clinics but not at control clinics. Overall satisfaction with services also increased only at intervention clinics but not at control clinics (Agha et al 2007:320). 
Another study aimed to evaluate end user satisfaction regarding the assistance to their needs during hospitalisation, in a general hospital of a city in the interior of Sao Paulo, demonstrate that end users were satisfied with the care rendered (Palva \& Gomes 2007:973). Sovd, Mmari, Lipovsek and Manaseki-Holland (2006:519) conducted a study to investigate which characteristics of health service quality are most likely to determine end user satisfaction with health services among adolescents in Mongolia.

Findings of this study by Sovd et al (2006:519) indicate that the strongest determinant to end user satisfaction is related to acceptability: adequate facility, physical environment, receiving adequate information about the health facility, and if the health facility was confidential (that is, other people didn't know the services the end user received). Additionally, end users who said they received some interruptions, either by other health workers or end users were significantly less likely to be satisfied with the services (Sovd et al 2006:519). Fonseca, Gutierrez and Adami (2006:656) conducted a study to evaluate the satisfaction level of cancer care end users at Adult Chemotherapy Ambulatory of Hospital Sao Paulo.

Findings showed that the level of satisfaction manifested by the end users was related mainly to the organisation accessibility, the welcoming environment and the assistance process, in its professional/end user interaction and the technical performance dimensions. Banks and Halasa (2005:1), in an end user satisfaction study from Jordan, report that end users were generally satisfied with the admissions process, safety and privacy issues, and the cleanliness of their rooms, and they were very impressed with the technical knowledge of health care workers. They expressed more dissatisfaction with the cleanliness of the health facility's common areas, comfort issues such as noise level on the ward at night, and, especially among end users in obstetric and gynaecology department, management of pain. They also pointed out that many health care workers fail to communicate with them about medical issues such as diagnosis, treatment options, length of health facility stay, and length of recovery (Banks \& Halasa 2005:1).

Despite the array of satisfaction reports seen from reviewed literature above, Collins, Nicolson and Bowns (2000:83) warn that it is worthy of note that in perception or end user satisfaction studies, end users' opinions may be partly as a result of their 
reluctance to say the wrong thing or complain for fear of unfavourable treatment in the future. It may also be because of the fact that some end users tend to agree with statements of opinion regardless of content, that is, the acquiescent response set (Collins et al 2000:83).

In this vein, Coulter (2010:4) argues that end users' attitude to the likely benefits and risks of their illness should be a key factor in ensuring an informed choice of treatment. Use of evidence based decision aids has been shown to lead to improvements in end users' knowledge, better understanding of treatment options, more accurate perception of risks, and reduced demand for elective procedures (Coulter 2010:4). Another study found that end users who prefer a more active role are less satisfied when their health care workers do not support their preference (Beach et al 2007:1119).

In conclusion, the literature of the perceptions of end users on quality indicated:

- $\quad$ effective orientated end users could reduce the frequency of health facility visits

- knowledge explosion among support group membership could create opportunities for greater decision-making regarding their care

- $\quad$ health facilities continuously improve quality and processes to meet the changing needs of end users

\subsubsection{End users' perception concerning effective and cost-efficient ART service provision}

Creel et al (2002:1) report that high-quality services ensure that end users receive the care that they deserve. Furthermore, providing better services at reasonable prices attracts more end users, increases the use of health services, and reduces the number of medical complications. Several impact studies have shown that improving the quality of health services increases health service uptake (Creel et al 2002:1). Studies in Bangladesh, Senegal, and Tanzania for example, showed that women's contraceptive use was higher in areas where end users felt that they were receiving good care than it was in areas with lower-quality health facilities (Creel et al 2002:1). Studies also indicated that improving the quality of health care programmes (such as ART) benefits other health services as well, in part by encouraging end users to seek higher-quality 
services for all of their health care needs (Creel et al 2002:1). In addition, improvements to health care facilities can enhance the quality of care for a wide range of adult and child health care needs.

Providing high-quality care also makes sense for service providers, since improving basic standards of care attracts more end users, reducing per capita costs of services and ensuring sustainability (Creel et al 2002:1). For example, the Bangladesh Women's Health Coalition attracts end users by providing a mix of services, so that end users can use a visit for more than one purpose, and by having well-trained paramedical personnel, rather than physicians, perform pelvic exams, intrauterine device (IUD) insertions, and menstrual regulation services.

The high volume of end users has enabled the programme to distribute its fixed costs over a larger number of end users, allowing the coalition to serve more people at a lower cost (Creel et al 2002:1). Liu and Wang (2007:266) also report that end user satisfaction can significantly affect a health facility's revenues and marketing plans. Adding that satisfied end users tend to remain loyal to that particular health facility and are likely to use the health facility's services again.

TQM is expected to improve quality as measured by improved end user satisfaction and working environment. Mehrabi et al (2008:566) indicated positive changes in end user satisfaction following implementation of TQM in investigated health facilities. Mehrabi et al (2008:566) lament that satisfaction of end users with health facility services gained a score of 4.16 out of 5 .

\subsection{CONCLUSION}

This chapter reviewed literature relevant to service users' perceptions regarding quality of antiretroviral therapy services. The perceptions of end users discussed included the quality of ART service provision and users' expectations thereof. This chapter also reviewed the concept of $\mathrm{CCM}$ as a framework for the guidelines obtained in the study.

Six elements of the Wagner's chronic care model emerged from the literature review (Singh and Ham 2006:5), composed of community resources; the health care system; end user self-management; decision support; delivery system redesign; and clinical 
information systems. It also emerged from the literature that end users constantly identify quality gaps regarding their care and develop improved requirements and expectations for their care. Against this background, health care workers will strategically review these quality gaps and use best practice guidelines to meet end users' requirement and expectations for improved quality of care.

Review of related literature against perceptions of end users concerning quality of ART service provision highlighted courtesy services of doctors, nurses and other health care workers; adequacy of infrastructure and environmental standards, and coordination and linkages between all role players for comprehensive service delivery as relevant to the current study. The literature related to the perceptions of end users concerning quality of antiretroviral counselling services outlined that end users cooperate (in support groups) with enthusiasm to improve the quality of life of peers and health care workers empathise with end users in order to build confidence and enhance satisfaction/quality of life.

Reviewed literature against the perceptions of end users concerning quality of antiretroviral therapy pharmacy services revealed that uninterrupted, adequate supply and availability of ARV and other drugs are necessary for treatment and management of complications. It also revealed that competent and adequate pharmacists deliver quality pharmacy services in ART clinics. It added that the supply chain management of laboratory reagents and drugs are useful for treatment success. The literature also indicates that lifesaving ART programme is fundamental in public health services.

Literature relevant to perceptions of end users concerning quality of antiretroviral therapy laboratory services support maintenance of linkages with relevant facilities to obtain specialised diagnostic services, and ensuring uninterrupted laboratory services by reducing equipment down time and laboratory commodities stock out.

Literature related to the perceptions of end users concerning antiretroviral pharmaceuticals based on material characteristics revealed that the health facility should be responsive to the needs of end users through leadership and policies, and to help end users emerge satisfied from the health facility. 
Relevant literature on the perceptions of end users concerning their interpersonal relationship with health care workers revealed that health care workers and support group volunteers should strive to treat end users with courtesy and affability, to improve their quality of life. It also revealed that health facilities should promote opportunities for interaction of colleagues and end users during service delivery; and that health facilities should strengthen systems for end user complaints management.

Review of literature against perceptions of end users concerning respect for their choices/preferences for antiretroviral therapy services revealed that leadership is needed to implement innovative approaches to improve dialogue with health authorities. Dialogue, if well managed, will improve the quality of life of people on ART. Also, to ensure availability and provide opportunities for end users to choose from available lifesaving antiretroviral drugs

Literature relevant to the perceptions of end users concerning timeliness of health services revealed that inadequate treatment of end users could be related to inadequate resources. It also revealed that standardisation of health care processes and procedures is needed to promote workflow; and that standardised processes and procedures could reduce end user waiting time seeking health care.

Literature relevant to perceptions of end users concerning end user centeredness revealed that health facilities should ensure that any end user who turns up to the health facility benefit from full assessment of his/her current health status. It also contained that strengthened linkages between support groups and community based organisations could promote accessibility and availability of wrap around services. The literature recognised the essence of capacity building and motivational programmes as necessary to meeting the task requirement of PLHIV volunteers. It canvasses for integration of HIVIAIDS into regular health services, and encourage end users to attend regular clinics.

Relevant literature on perceptions of end users concerning end user safety indicated that infection prevention and control should be a priority in health facilities; and that a stigma free environment should be ensured for end users. 
The literature on perceptions of end users concerning quality of communication and information also connotes that knowledge and information improves relationships and quality of care. It calls for strengthened systems to promote dialogue between support group members and health facility leadership. It also calls for strengthened systems to promote dialogue within the support group membership.

Review of literature relevant to perceptions of end users concerning their rights to decision-making regarding their care revealed that stable end users could reduce their frequency of health facility visits. It also indicated that knowledge explosion among support group membership could create opportunities for greater decision-making regarding their care. It revealed that health facilities continuously improve quality and processes to meet the changing needs of end users.

The next chapter (chapter 3) will present the research methodology adopted to study quality of antiretroviral therapy in public health facilities in Nigeria and the perceptions of the end users. 


\section{CHAPTER 3}

\section{RESEARCH DESIGN AND METHOD}

\section{$3.1 \quad$ INTRODUCTION}

This chapter presents the research design and methods utilised in conducting this study. The chapter also presents methods of data analysis, ethical considerations and steps taken by the researcher to ensure trustworthiness of the research. Data were collected using unstructured descriptive FGDs, held in six locations of the six geopolitical zones of Nigeria. The study followed a qualitative research paradigm.

\subsection{RESEARCH SETTING}

This study took place in a natural environment where study participants usually held their support group activities, to depict their lived experiences; thus, their naturalistic setting. This is in line with Patton's (2002:280) stipulation, that qualitative studies are undertaken in a natural setting to be able to put the participants' experiences into context.

This study took place in six state public health facilities providing ART services, representing the six geopolitical zones of Nigeria. Specific states where the study took place include Yobe (located in the North-East zone of Nigeria), Sokoto (North-West), Federal Capital Territory (North-Central), Lagos (South-West), Edo (South-South) and Anambra State (located in South-East geopolitical zone of Nigeria). See Figure 3.1 showing map of Nigeria with the study sites marked. A cumulative number of over 20,000 end users attend ART services in the 6 study sites, with approximately 3,000 monthly attendances per site. Of this number, about 228 are new end users enrolled into the ART programme monthly, across all the 6 study sites (See Annexure $\mathrm{H}$ for the 2011 annual breakdown of cumulative number of end users who ever received ART in the 6 study sites. Different cultural practices are found in each geopolitical zone of Nigeria. For example, although there are three major languages spoken across all the six geopolitical zones of Nigeria, the north has a predominance of Hausa speaking 
people, while the South West has Yoruba and the South Eastern part of the country has a predominance of Ibo speaking people.

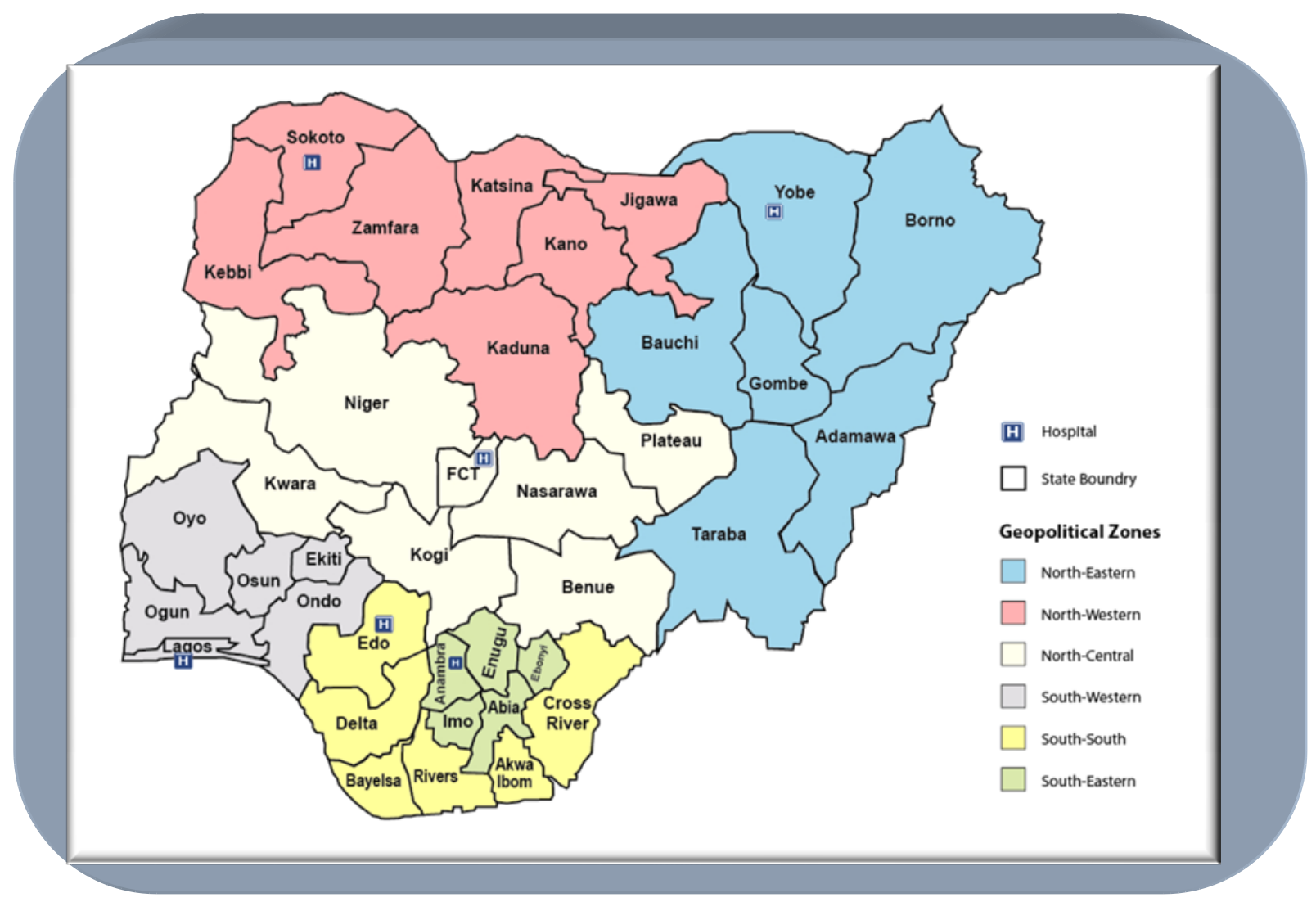

Figure 3.1 Map of Nigeria showing the six geopolitical zones and the six public health facilities used as research sites for this study

The selection of public health facilities from the six different geopolitical zones of Nigeria is supported by Moleki (2008:65) who assert that the lived experiences of participants coming from different settings enhance the richness of the data collected. The researcher visited all six study sites to get first-hand information on the natural setting of participants, and to be able to put the participants' lived experiences of the quality of ART service provision into context.

\subsection{RESEARCH DESIGN}

\subsubsection{Research paradigm/approach}

A qualitative paradigm was applied for this study. The qualitative research paradigm was chosen for this study because of its heuristic value, or its usefulness as a tool for 
exploration related to knowledge development. It often informs clinical practice by raising questions and providing fertile ideas for improving quality of care and prompting additional research activities. This study requires an understanding of the perceptions of end users concerning quality of ART service provision in public health facilities in Nigeria. It is obtainable through data collection and interpretation (qualitative research). Stommel and Wills (2004:442) define qualitative research as that research that eschews measurement and focuses on interpretive, non-numerical, narrative interpretations. Qualitative research aims to describe social phenomena and behaviours using rich contextual data that emphasise the subjective experiences of social actors (Malta, Maya, Clair, Freitas \& Bastos 2005:1426).

While qualitative studies use "open-ended" questions, quantitative studies use "closedended" questions. Chow et al (2009:441) elucidate that the open style of questioning in qualitative studies allows the end user freedom to comment on areas of care from which we infer end user satisfaction. With "open-ended" questions, an end user is asked to comment on an area of care. The end user responds by explaining in detail. He/she is then probed by the interviewer or FGD facilitator to continue to get deeper into the subject matter, until he/she gets to a point where there is nothing else to share (point of saturation). While the end users are openly sharing their perceptions regarding the question(s) under consideration, the investigator is at liberty to discover satisfaction and dissatisfactions with health care services. In qualitative studies, direct questions are also asked. Chow et al (2009:441) state that direct questions tend to serve as probes in qualitative studies, to discover satisfaction or dissatisfaction with areas of care that may not be mentioned with an open-ended question.

Stommel and Wills (2004:178) maintain that the main characteristics of qualitative research are as follows: qualitative research is oriented towards theory discovery and the development of a conceptual framework. It attempts exploration in a "naturalistic" way, under uncontrolled conditions. Its goal is to understand behavior or actions within their naturally occurring contexts. It focuses on behavioral or meaningfully understood action variables only. It has a tendency to focus on smaller samples. It has preference for open-ended, un-standardised, reactive data collection procedures. It is oriented toward completeness of description. It is open-ended to inquiry (reactive to preliminary results). It emphasises the uniqueness of individuals or special population groups, preferring narrative summaries or descriptions. 
"Closed" form of questioning in quantitative studies involves direct questions asked about satisfaction with specific services. Closed questioning gives more quantitative evaluations, but does not provide the situation the end user is referring to (Chow et al 2009:441). For example, a change from satisfied to dissatisfied may represent either an accumulation of small shifts in a separate component area or a large shift in a single component. Responses to this form of rating tend often to fall into two narrow bands, being only superficially indicative of high satisfaction levels.

Due to the obscurity of bringing out the inert perceptions of end users in quantitative methods, the qualitative methods of studying end user satisfaction remain the most viable option. Qualitative methods influence respondents to bring out new areas of thought that were never preconceived by the quantitative instrument developer/ researcher. It was against these merits of qualitative study methods that the researcher opted to use qualitative study approach in this study.

The qualitative research approaches are most appropriate for this study because they demonstrate the appropriate study architecture, strategy, and tactics for exploring the views of end users through focus group discussions (to the point of redundancy or saturation). However, Stommel and Wills (2004:291) conclude that although qualitative research is indispensable, it can be criticised for placing too much trust in the credibility and integrity of the individual researcher. In the ordinary scientific canon, methods that produce results that cannot be replicated may be heuristically useful, but they do not meet the standards of objectivity, which demand that results can be corroborated by other independent researchers. However, the researcher enhanced the trustworthiness of the research through strategies described in Section 3.5.

\subsubsection{Research design}

A descriptive and explorative research design was used in this study. This research design was preferred for this study because it provides an accurate and real-life situation, experiences and characteristics about the end users participating in this research. Polit and Beck (2004:528) define descriptive research as research studies that have as their main objective the accurate portrayal of the characteristics of persons, situations, or groups, and the frequency with which certain phenomena occur. Thus, 
descriptive and explorative research is designed to summarise the status of some phenomena of interest as they currently exist, introducing no new treatments or changes. The use of a descriptive and explorative research design to this research is justified on the basis of the flexibility of the design in promoting descriptions or exploration of the quality of antiretroviral therapy in public health facilities in Nigeria and the perceptions of the end users.

Descriptive and explorative research designs, when compared with the formal measurement models used for quantitative scaling and the efforts to standardise data collection procedures for quantitative measures, qualitative (descriptive and explorative) design appear to lack rigour and objectivity. However, it would be erroneous to conclude that clinical research can "do without" the flexible methods provided by qualitative research approaches (Stommel \& Wills 2004:291). In the current study the descriptive/explorative design will provide a platform for the participants to present their perceptions concerning quality of ART in Nigerian public health facilities to the point of saturation, or the point whereby they have nothing else to contribute to the research question.

\subsection{RESEARCH METHOD}

This section presents systematic and detailed information that will allow a reader to replicate or otherwise evaluate the study findings (Stommel \& Wills 2004:420). It provides a detailed description of the key characteristics of the study sample, including the inclusion and exclusion criteria used in the study. It also presents the ethical issues in the protection of human subjects. It provides a detailed description of methods used to collect data, including steps taken in data collection, data analysis and ensuring trustworthiness of the data.

\subsubsection{Population, sample and sampling}

\subsubsection{Population}

Stommel and Wills $(2004: 297,441)$ define population as any universe of subjects, cases, units, or observations containing all possible members. Polit and Beck (2004:534) define a population as the entire set of individuals (or objects) having some 
common characteristic(s) sometimes referred to as universe. The population for this study was the end users of health services in Nigeria. This population was ideal for the study because they form the core reason for the existence of public health facilities in Nigeria.

Target population, according to Stommel and Wills (2004:444), refer to the population of all potential study units that meet the study inclusion criteria (that is, in whom or which the researcher is interested). Polit and Beck (2004:537) define target population as the entire population in which the researcher is interested and to which he or she would like to generalise the results of a study.

The target population for this study includes end users of health services in each of the six geo-political regions of Nigeria. The accessible population includes all end users of ART services in government designated ART centres in public health facilities in Nigeria.

Stommel and Wills (2004:299) define study population as the subset of the target population that are accessible to the researcher, at least in principle, if there are sufficient resources. These accessible study populations are more limited in time and space and are also defined in terms of specific inclusion and exclusion criteria. Eligibility criteria refer to pre-determined benchmarks that must be attained before one is qualified to be selected as a sample. Thus, for this study, the researcher included end users only (male or female) who met the following inclusion criteria:

- $\quad$ attending ART in the government designated centre in a public health facility in Nigeria

- $\quad$ at least 18 years old

- $\quad$ attending the ART programme for at least 6 months on a continuous bases

- $\quad$ willing to provide written consent to participate in the study

For the purpose of this study, the researcher purposively approached/included participants who met eligibility criteria for inclusion in the FGD, and also gave written informed consent to participate. Those who did not meet the eligibility criteria or refused to grant consent to participate were excluded from the study. Purposive sampling technique was used to obtain a study sample in each of the 6 health facilities. The study 
sites refer to the 6 health facilities that were units from a larger population of health facilities, while the study sample refers to the end users that participated in the study (Stommel \& Wills 2004:443).

\subsubsection{Sample and sampling techniques}

A purposive sampling technique was used in this study. The purposive sampling technique was the preferred approach for this study because it enables the selection of most readily available end users for the study. This sampling method was preferred after the researcher verified from health facility records that end users listed in the ART records were necessarily not still available for continued care in the health facility. Some were dead, transferred out, lost to follow up or lost to other unknown reasons; thereby, creating difficulties in achieving accurate representativeness of samples. Polit and Beck (2004:527) refers to purposive sampling as the selection of the most readily available persons (or units) as subjects in a study; also known as accidental sampling. Stommel and Wills $(2004: 436,437)$ on the other hand, view purposive sampling as a nonprobability approach to selecting participants based on their (easy) accessibility to the researcher.

Procedurally, the researcher randomly selected one public health facility per geopolitical zone in Nigeria. Thus, of the 6 official geopolitical zones in Nigeria, 6 health facilities providing ART were randomly selected to participate in the study. A public health facility was selected per geopolitical zone to capture end users' diverse perceptions concerning quality of ART in Nigerian public health facilities. The researcher was supported by trained research assistants and local leaders of support group of people living with HIV and AIDS in each study location to identify end users attending ART in the public health facilities. End users attending ART were approached by the support group leaders and screened for eligibility to participate in the study. Eligible end users were sequentially enrolled into the study based on first-come, first-eligible, first-enrolled bases, until the required number of 8-12 persons per FGD was achieved. End users who failed to meet the inclusion criteria listed in section 3.3.1.1, were excluded from the study.

The main problem with purposive sampling is that members of such a sample may have some characteristics, often unrecognised, if not explicitly studied or thought about, that distinguish them from the overall target population. Membership of the study sample 
always involves a substantial element of self-selection, thus, subjectivity or bias. It is sometimes difficult to gauge the extent to which findings from a particular study are applicable to broader populations.

Table $3.1 \quad$ Number of focus groups held per location

\begin{tabular}{|l|l|l|l|l|l|l|l|}
\hline & $\begin{array}{l}\text { Health } \\
\text { facility } \\
\text { 'A' }\end{array}$ & $\begin{array}{l}\text { Health } \\
\text { facility } \\
\text { 'B' }\end{array}$ & $\begin{array}{l}\text { Health } \\
\text { facility } \\
\text { 'C' }\end{array}$ & $\begin{array}{l}\text { Health } \\
\text { facility } \\
\text { 'D' }\end{array}$ & $\begin{array}{l}\text { Health } \\
\text { facility } \\
\text { 'E' }\end{array}$ & $\begin{array}{l}\text { Health } \\
\text { facility } \\
\text { 'F' }\end{array}$ & TOTAL \\
\hline North-East & 1 & & & & & & \\
\hline North-West & & 1 & & & & & \\
\hline North-Central & & & 1 & & & & \\
\hline South-East & & & & 1 & & & \\
\hline South-West & & & & & 1 & & \\
\hline South-South & & & & & & 1 & $\mathbf{6}$ \\
\hline
\end{tabular}

Table $3.2 \quad$ Number of participants in focus groups held per location

\begin{tabular}{|l|l|l|l|l|l|l|l|}
\hline & $\begin{array}{l}\text { Health } \\
\text { facility } \\
\text { 'A' }\end{array}$ & $\begin{array}{l}\text { Health } \\
\text { facility } \\
\text { 'B' }\end{array}$ & $\begin{array}{l}\text { Health } \\
\text { facility } \\
\text { 'C' }\end{array}$ & $\begin{array}{l}\text { Health } \\
\text { facility } \\
\text { 'D' }\end{array}$ & $\begin{array}{l}\text { Health } \\
\text { facility } \\
\text { 'E' }\end{array}$ & $\begin{array}{l}\text { Health } \\
\text { facility } \\
\text { 'F' }\end{array}$ & TOTAL \\
\hline North-East & 12 & & & & & & \\
\hline North-West & & 12 & & & & & \\
\hline North-Central & & & 8 & & & & \\
\hline South-East & & & & 10 & & & \\
\hline South-West & & & & & 10 & & \\
\hline South-South & & & & & & 12 & $\mathbf{6 4}$ \\
\hline
\end{tabular}

\subsubsection{Method of data collection}

\subsubsection{Data collection process}

Data collection was executed in three phases. The phases were derived from the work of Patton (2002:213), namely preparatory phase, gaining access in the field and leaving the field.

\section{- $\quad$ Preparatory phase}

The preparatory phase involved gathering adequate information about the investigation, including the rigorous integration of the research method, preparing the interview guide and identifying the target population. It also involved the researcher's technical, 
emotional, psychological and logistical preparedness for the investigation. The preparatory phase also included identification of workplace colleagues, experienced in qualitative research methods, to assist in data collection (as research assistants). These research assistants were given refresher training in facilitating focus group discussions, ethical issues in research/data collection and managing relationships with research participants and stakeholders. The research assistants in turn, identified other experienced qualitative researchers in the same workplace and retrained them as note takers/audio tape managers per location. While the training for research assistants were conducted by the researcher via telephone conference facilities, those for note takers were conducted face to face, by the research assistants.

\section{- $\quad$ Gaining access in the field}

The researcher randomly identified a public health facility per geopolitical zone of Nigeria. For each facility, he passed telephonic information through his workplace colleagues in the respective geopolitical zones to make logistical arrangements for FGDs to be held in identified public health facilities. The public health facilities and leaders of the support group of people living with HIV and AIDS in the health facility were in turn informed of the study by the researcher's colleagues, who already had good rapport with people in the health facilities.

The researcher's colleagues presented ethical clearance/approvals from the Department of Health Studies Ethics and Research Committee, UNISA and National Research Ethics Committee (NREC) and the researcher's background and interest in the study, to secure cooperation and venue for the FGD, from the health facility management. The researcher's colleagues also advocated to the leaders of the support group of people living with HIV and AIDS in the public health facility to avail their members who consent to participate in the study to do so without fear or favour.

Once the date and venue of the FGD was confirmed, the research assistants went to the study location. The use of pre-existing relations of trust between the researcher's colleagues working in the study locations removed barriers to entering the research setting (Lofland \& Wilkblud 2001:69; Moleki 2008:76). A quiet area was secured in the public health facility to hold the FGD. The tape recorder was checked for functionality prior to the FGD. 
The researcher introduced himself and his colleague, describing the role of each - the researcher served as facilitator for the FGD while the note taker took notes and managed the tape recorder. Further, he shared information about self as a senior public health official, and that results of the study could be used to develop user focused best practice guidelines for ART. This made it easy for both the researcher and the participants to engage in the FGD.

\section{- $\quad$ Leaving the field}

This stage marked the end of the FGD held on the field (inquiry). It involved the 'when' and the 'how' of terminating the study as well as addressing the related ethical and emotional questions (Moleki 2008:78). The following criteria guided the appropriate conclusion of the fieldwork. Firstly, the field could be left only when the researcher was satisfied that the data had maximally highlighted the experience and explained the key concepts, adequately addressed the objectives and satisfactorily answered the research question. This was with an understanding that the researcher can return for more information or clarity if this was required. Secondly, the field could be left when the actions under scrutiny become predictable, when there are no new developments, insights or knowledge forthcoming indicating data saturation (Moleki 2008:78). Having satisfied these two basic guiding principles for termination of the fieldwork, the researcher thanked the participants, health facility management and his colleagues who facilitated the successful hosting of the FGDs.

\subsubsection{Data collection method}

Unstructured focus group interviews were conducted in this study. The focus group approach was preferred for this study for two major reasons. One was the hope that participants are more likely to "reveal" themselves in unstructured interperceptions, providing "unfiltered" personal perceptions of their experiences concerning quality of ART. Thus, an approach that allows interviewees to use their own language was viewed as a "bias-free" account of their experiences. The second reason was FGD's potential to provide a rich or thick, detailed data, consisting mainly of verbal accounts, ideas and qualities, highly specific end users' perceptions concerning quality of ART (Burns \& Grove 2001:738; Patton 2002:342; Moleki 2008:78). 
Stommel and Wills (2004:445) define unstructured interview as an interview style in which the flow and content of the interview are largely determined by the interactions between interviewer and interviewee. Stommel and Wills (2004:438) define focus group interview/discussion as a group session for the purpose of conducting a semistructured, qualitative interview in which a small group of participants (6 to 12 people) discuss among each other a series of pre-determined focus questions provided by the researcher. Polit and Beck (2004:529) on the other hand, explain FGD as an interview in which the respondents are a group of 10 to 20 individuals assembled to answer questions on a given topic. Family Health International (FHI) (2005:38) elucidate that FGDs are used to gather targeted information from a group of people via open-ended questions.

A decision to use FGDs instead of in-depth interviews is driven by the need to experience albeit in an artificial setting, the experiences and perceptions of the participants. In-depth interviews often require face-to-face interactions between an interviewer and an interviewee (one-on-one), while FGDs require a facilitator versus a group composed of 8-12 participants. In-depth interviews perform less well when the emphasis and purpose of the study are an exploration of a phenomenon or a subject area about which relatively little is known. In-depth interviews also perform below expectations on subjects requiring personal perceptions or views of individuals regarding a given subject.

Subjective phenomenons, such as end user satisfaction require FGD in order to explore shared experiences of participants. In-depth interviews, whether face-to-face or via telephone, involve the verbal exchange of information between two persons, the interviewer and the interviewee, thereby, making it expensive to cover an acceptable sample size. For example, it is simply not realistic or defensible to draw a random sample from a geographically widely distributed target population, such as PLHIV in all health facilities in Nigeria, and to send interviewers to meet with all selected respondents. The time and travel costs alone for such a project would be very large (Stommel \& Wills 2004:255).

When in-depth interviews are conducted face-to-face, respondents may feel uncomfortable and embarrassed if they are asked to answer questions about socially 
sensitive topics, such as their perception about the quality of care provided by a team of nurses or doctors in a certain health facilitity. In this case, the end user will either provide false responses inorder to continue to receive life saving antiretrovirals in the health facility, or refuse to answer for fear of victimisation.

Such a situation suggests the necessity of open-ended questioning in focus groups, to bring out salient issues that would otherwise not be said in in-depth interviews, due to lack of confidence, or forgetfulness (in some cases). In FGDs, points raised by one participant stimulate the other to build upon it, thereby, facilitating debates and in-depth progression into the subject matter. Successive open-ended questions and answers drills participants to continue to think deeper, reflect on the subject matter and bring out further salient points that would otherwise, not be remembered (or said) in in-depth interviews. The facilitator follows through with probing questions until no further responses are generated from the respondents. This is the point of data saturation in FGD.

Another benefit of conducting FGD is that it provides the researcher an opportunity to observe respondents' un-said expressions, such as body language expressing their perception, group dynamics, sitting arrangement, mode of appearance, and so forth. Group dynamics and interaction among respondents can not be observed in in-depth interviews, since such interviews are usually conducted one-on-one. Stommel and Wills (2004:285) argue that the use of any set of predetermined questions as obtainable in indepth interviews, even if derived after extensive pretesting with members of the target population, imposes explicit preconceptions. Thus, only an approach that allows FGD participants to use their own language should be viewed as a "bias-free" account of their experiences.

The selection of FGD members requires careful decisions to optimise the usefulness of the focus group. For most purposes, groups of 6 to 10 members are optimal (Stommel \& Wills 2004:284). The moderator stimulates the exchange of ideas and encourages debates.

The FGD methodology provides information, but it does not produce quantifiable data and the findings cannot be generalised to a larger population. The findings will not include numbers or percentages. Instead, FGDs capture broad themes that convey 
participants' experiences and perspectives and uncover why people think and feel as they do. The most important element of the FGD data collection method is the quality of the interaction among FGD participants. The moderator skilfully guides participants through a series of questions intended to trigger a free-flowing discussion that includes the inert perceptions of participants on the topic under study and debates over suggested ideas. It is this dynamic aspect of FGDs that result in data emerging from critical and creative inputs that can guide needed actions. Resistance to an idea that would have remained hidden during one-on-one interviewing may be uncovered during an FGD.

Procedurally, an FGD of 8-12 persons each was conducted with end users attending ART in public health facilities in each of the 6 geo-political zones of Nigeria. A total of 64 people participated in the FGDs across the six locations. While the FGDs in the North East, North West and South-South regions were composed of 12 participants each, those in the South East and South West were composed of 10 participants each. The FGD held in the North Central zone was composed of only 8 participants, following withdrawal from participation by two end users.

\section{Preparation of focus group discussion on site}

In keeping with the general intent to reduce the researcher's control over the FGD and understand the perceptions of end users regarding quality of ART service provision, the FGDs took place in settings that were familiar to the participants. Thus, participants were met in their usual venues for holding support group meetings. In each location, the research team ensured that all 8-12 participants expected for the FGD were around and seated, before the FGD commenced. The facilitator coordinated FGD participants to adopt a "C-shaped" seating arrangement, with each one facing the entire group members and the research team alike. This seating arrangement ensured eye contact between one participant and the other, and between participants and the research team (facilitator and note taker). While a mixed seating arrangement was observed between male and female participants during the FGD sessions in the south (south-south, southwest, south-east) and north-central zones (study site was in the Federal Capital Territory), the researcher observed that participants in the north-east and north-west zones adopted a sitting arrangement with males concentrated on one side of the room and the female on the other side. This depicts dominance of a masculine society, where women do not freely mix with the men. Therefore, this society could miss out on the 
characteristic dominant values in a feminine society which are caring for others and quality of life (Mooij and Hofstede 2010:89). The dominant values in a masculine society are achievement and success (Mooij and Hofstede 2010:89).

\section{During the focus group discussion}

The FGD facilitator opened discussion sessions by welcoming participants to the group. This was followed by conducting self introductions, beginning with the research team. Once everyone had introduced themselves, the researcher explained the purpose of the FGD and obtained written consent from participants. Participants who felt uncomfortable to participate were advised to leave at any point of the FGD process. All participants provided consent to participate and stayed through the process, except two persons in the north-central zone, who declined participation.

Once the FGD began, the researcher observed initial reluctance in the participants, to express themselves during the first 5-7 minutes of the FGD, until thereafter when full participation was noticed. The FGD sessions across all 6 study sites were noted to be participatory, with participants actively responding to all questions asked. Each question asked was followed by answers from participants. Where appropriate, the facilitator asked probing questions, and in some instances, direct questions, to clarify comments made and harvest more responses from the study participants. Questions sparked up debates, reflections, "food for thought" and rich responses among participants, giving rise to further information gathering by the researcher. The facilitator made notes of data from a synergistic group process that gave group members a chance to prompt or enhance their own thinking on the subject in response to other group members' contributions. The facilitator consistently made efforts to remain focused on the topic, encouraged participants to speak out, and avoided domination of the group by any single participant.

Four FGD sessions (held in the south-west, south-east, south-south and north-central geopolitical zones) were conducted in English language, while two sessions (held in the north-west and north-east geopolitical zones) were conducted in Hausa language. The FGDs that took place in Hausa language were transcribed and translated into English language. Transcription and translation for the FGD held in the north-east was made by the research assistant/FGD facilitator who was very fluent in both spoken and written 
Hausa and English languages, while the one for the north-west was transcribed and translated by the researcher, with assistance from the note taker.

To achieve the purpose of the study and explore the research question, the researcher took control of the FGDs to facilitate discussions within the scope of the study, while allowing participants to freely share their perceptions concerning the quality of ART in the health facility. To ensure this, a FGD guide was developed prior to the FGD.

The FGD guide consisted of three sections, which included the following:

Section 1 of the FGD guide provided welcome remarks to build rapport between the researcher and the participants, including information on the objectives of the study and signing of consent forms. This section also comprised of the bio-data of participants, including age, gender, occupation and highest educational attainment. Data was also sought on participants' duration on the ART programme in the public health facility.

Section 2 of the FGD guide comprised of the main research question:

1. What are the perceptions of end users regarding the quality of ART services received in public health facilities in Nigeria?

In order to check whether what participants say they do is the same as what they actually do (Mulhall 2002:307).

Details of the main questions are contained in 'Annexure F' of this thesis. Probing questions, as guided by the information elicited during the FGDs were also used. The probing questions were however not prepared in advance.

Section 3 consisted of the conclusion whereby the researcher summarised the main points noted during the FGD and confirmed from the participants that they were correct and true reflection of the FGD.

\subsubsection{Ethical considerations}

The Belmont Report became the foundation for research regulations in federally 
sponsored research in the United States, thus, merits special mention (Stommel \& Wills 2004:377).

The following ethical considerations were followed in the course of this study:

The ethical considerations of the study were observed, where upon an informed consent form was provided for the participants to sign. Participants were also informed that the FGD would be tape-recorded following their permission and the researcher would take notes during the interview. The ethical approval to undertake the study was obtained from the Department of Health Studies Ethics and Research Committee, at the University of South Africa and from the NREC, Federal Ministry of Health, Abuja, Nigeria. The approval ensured that the research proposal had been fully scrutinised,

adding credibility to the quality of the study. Ethical issues were also explained and participants were assured that they could withdraw from the study or refuse to answer any question, which might seem to be of discomfort to them with no penalties.

Once the FGD began, the researcher engaged the note taker to assist in taking detailed notes and managing the audio tape recorder. The researcher also took brief descriptive summaries from observations made that included thoughts and feelings about the focus group interviews, descriptions of the participants, and sketches of the seating arrangements. This information was used as part of an audit trail in support of the confirmability of the study findings and for triangulation with the note takers' briefs in data analysis. Each FGD lasted 60-90 minutes, conducted in English language, except in the North East and North West regions, where the FGDs were conducted in Hausa language and translated into English.

\subsubsection{Protecting the rights of the participants}

\section{Non-discrimination}

The researcher avoided discrimination against end users attending ART on the basis of gender, race, ethnicity, or other factors that may jeopardise the validity and reliability of the study results. This was demonstrated by giving equal opportunities to all end users attending ART in public health facilities to participate in the study at their free will. 


\section{Beneficence}

The researcher did no harm, refrained from exploitation of participants, and promoted both individual and societal benefits that were directly related to participation in this research. All study participants were assured that they will not be exposed to any physical harm during the study. The researcher also provided full protection to study participants from any physical exposure or stress, including conducting the FGD in participants' natural environmental setting and informing them in advance, to feel free to exit the FGD at any time they felt uncomfortable to continue. The benefits of the study include harvesting end users' needs, perceptions and expectations for quality ART. Therefore, results of the study will be used to improve quality of ART, using end users' perspectives of quality ART.

\section{Respect for persons}

The researcher respected the rights of end users attending ART, both for selfdetermination (autonomy) and the right to full disclosure (fully informed consent for research participation). The rights of the end users to decide to participate in the study were respected by the researcher, based on the ethical principle of self-determination and the expected professional obligation of the researcher to truthfully grant full disclosure of information relevant to the study. To enable participants make informed decision to participate in the study, the researcher provided detailed explanation on the study, including but not limited to the purpose of the study, consequences of participation or refusal to participate, and any possible gains or risks associated with participating in the study. In addition, the researcher informed participants that they have the right to withdraw from the FGD at any time they deemed fit, and that their withdrawal will be of no consequence to them, including their continued participation in the ART programme in the health facility. Following the full disclosure of information regarding the study, participants were asked to voluntarily sign a written consent to participate in the study. The researcher witnessed the signing, and countersigned as witness.

\section{Justice}

The researcher respected the rights of the end users to privacy and the right to fair treatment in the context of research participation. The privacy of participants regarding any information relevant to them, and their identity were maintained and jealously safeguarded by the researcher. No section of the research report contained any 
information that could be used by any reader to contemplate the source of such information.

\subsubsection{Protecting the rights of the institutions}

\section{Legality}

The researcher knew and obeyed relevant laws and institutional and governmental policies regarding research, protection of human subjects, and any other ethical consideration relevant to this study.

\section{Integrity}

The researcher kept promises and agreements with the research supervisors, institutions providing access to records and any other authority relevant to this study; and acted with sincerity and strived for consistency of thought and action.

\section{Openness}

The researcher shared data, results, ideas, tools, resources with relevant persons and institutions. He was open to criticism and new ideas.

\section{Respect for intellectual property}

The researcher honoured patents, copyrights, and other forms of intellectual property. He did not use unpublished data, methods, or results without permission. He gave credit where credit was due and gave proper acknowledgement or credit for all contributions to the research. The researcher never plagiarised.

\section{Confidentiality}

The researcher protected confidential information obtained from the public health facilities; such as end users' records, names of public health facilities participating in the study and names of health facility workers cited by study participants. The researcher did not include any information in the research report that could be used by any reader to contemplate the identity of specific public health facilities used for this study.

\section{Responsible mentoring}

The researcher helped to educate, mentor, and advise students, interns and colleagues in the workplace who were willing to do research. He promoted their welfare and 
allowed them to make their own decisions.

\subsubsection{Scientific integrity of the research}

\section{Honesty}

The researcher strived for honesty in all scientific communications, by honestly reporting data, results, methods and procedures, and publication status after the thesis is accepted by the University. The researcher did not fabricate, falsify, or misrepresent data. He did not deceive colleagues, granting agencies, or the public on any part of the subject matter being studied.

\section{Objectivity}

The researcher strived to avoid bias in data analysis, data interpretation, peer review, personnel decisions, grant writing, expert testimony, and other aspects of research where objectivity was expected or required. He avoided or minimised bias or selfdeception. To ensure objectivity, the researcher shared the transcripts with another experienced researcher to independently do an analysis and compared notes thereafter. The researcher also did a peer debrief with other research colleagues in the workplace. The researcher discloses that he has no personal or financial interests that may affect this research study.

\section{Carefulness}

The researcher avoided careless errors and negligence; carefully and critically examined own work and the work of his peers. He kept good records of research activities, such as data collected, research design, and correspondence with agencies, supervisors or journals.

\section{Responsible publication}

The researcher published the final product of this research in order to advance research and scholarship, not to advance just his own career, ensuring to avoid wasteful and duplicative publication.

\section{Respect for colleagues}

The researcher respected his colleagues and treated them fairly. He acknowledged all 
who made useful contributions towards the success of this research study.

\section{Social responsibility}

The researcher strived to promote social good and prevented or mitigated social harms through research, public education, and advocacy.

\section{Competence}

The researcher maintained and improved his own professional competence and expertise through lifelong education and learning; and took steps to promote competence in science as a whole.

\subsubsection{Method of data analysis}

The analysis of qualitative data began in the field, during data collection, using the unstructured FGD. The data gathered was analysed on the spot to shape the on-going data collection process. Pope, Ziebland and Mays (2000:114) note that this sequential analysis or interim analysis has the advantage of allowing the researcher to refine questions on the spot and pursue emerging avenues of inquiry in further depth. Such continuous analysis is almost inevitable in qualitative research: because the researcher is "in the field" collecting the data, it is impossible not to start thinking about what is being heard or seen.

Once the researcher had left the field, the textual data (in the form of field notes or transcripts) was explored through content analysis. Pope et al (2000:114) argues that deductive analysis is less common in qualitative research but is increasingly being used, for example in the "framework approach."

To address issues of trustworthiness of qualitative research, the researcher used both manual and electronic software methods of data analysis. First, the framework approach (manual method) was used in this study. The framework approach was selected as the preliminary method of choice for this study because it reflects the original accounts and observations of the people studied (that is, "grounded" and inductive), though, it starts deductively from pre-set research purpose and objectives. It was also a preferred choice because the analysis was designed so that it can be viewed 
and assessed by people other than the primary analyst, required to support the dependability of the study.

Once the themes and sub-themes were identified using the framework approach, the analysis was repeated all over again using the Weft qualitative data analysis (Weft DQA) version 1.0.1 software, to validate the results obtained from the framework approach. Details of both the manual and software methods are described below.

\subsubsection{Stages of data analysis}

Data analysis using the framework approach. Pope et al (2000:116) suggest the use of five stages of data analysis in the framework approach, and were used in this study as follows;

Familiarisation. The researcher was substantially engaged in immersion of the raw data by listening to tapes, reading transcripts, studying notes and so on, in order to list key ideas and recurrent themes. The researcher also built upon existing relationship with the participants to strengthen more valid and meaningful rapport.

Through this process, the transcript was returned to the participants to review, add to, or otherwise revise transcripts of their study responses, or to correct the researcher's interpretation of the meaning of the data. The researcher also shared the transcript with experienced research colleagues in the workplace to review the entire process, proffer suggestions and reach consensus on the best way to improve the data analysis. This was done with all dedication, until full familiarity was achieved and the key themes identified.

The researcher shared the transcript for one of the FGDs with another experienced qualitative research consultant (who served as note taker on the field) to do an independent analysis. The analysis was then checked for comparability and similarity, and discrepancies were resolved.

Identifying a thematic framework.

The researcher engaged in identifying all the key issues, concepts, and themes by which the data was examined and referenced. This included the aims and objectives of 
the study as well as issues raised by the respondents themselves and perceptions of experiences that recurred in the data. The end product of this stage was a detailed index of the data, which labelled the data into manageable chunks for subsequent retrieval and exploration.

\section{Indexing}

The researcher applied the thematic framework or index systematically to all the data in textual form by annotating the transcripts with numerical codes from the index, and supported by short text descriptors to elaborate the index heading. Single passages of text were made to encompass a large number of different themes, each of which was recorded in the margin of the transcript.

\section{Charting}

The researcher rearranged the data according to the appropriate part of the thematic framework to which they relate, forming charts. The charts contained distilled summaries of perceptions and experiences. Thus, the charting process involved a considerable amount of abstraction and synthesis.

\section{Mapping and interpretation}

The process of mapping and interpretation was influenced by the results of manual and Weft QDA version 1.0.1 software analysis, as well as the original research objectives. This is discussed in more detail, after data analysis using the Weft QDA 1.0.1 software, below.

\section{Data analysis using the Weft QDA version 1.0.1 software}

Before the researcher did coding and analysis using the Weft QDA version 1.0.1 software, he analysed the entire scripts manually so that he could get an indication of the codes, sub-themes, themes and content of the analysis. Results of data analysis using the framework approach were shared with the researcher's supervisor, who made useful comments and requested for a repeat using select electronic software, to increase the trustworthiness of the qualitative research findings.

The researcher then engaged in a search of open source software and found the Weft QDA version 1.0.1 software, which was accompanied by a user's manual for self-study. The Weft QDA version 1.0.1 is a software tool for the analysis of textual data such as 
interview transcripts, documents and field notes. It is available free under a public domain licence.

The researcher downloaded and automatically installed the Weft QDA version 1.0.1, using rubygems. Weft QDA version 1.0.1 software was then run on c:/ruby/bin/weft_qda.rb using Windows XP. The researcher trained himself using the Weft QDA version 1.0.1 software user's manual and the help functions of the Weft QDA version 1.0.1 software. He finally appreciated that the software fitted the research purpose, selected methodology and was user friendly. Of particular interest was that Weft QDA version 1.0.1 software offers a generic set of facilities for working with text documents, and does not make any particular assumptions about how to think about and generalise from data.

The researcher started a new project by choosing New Project from the File menu. While the Weft QDA version 1.0.1 software project was in open mode, he imported the original research transcripts in plain text format, from the File menu of the Weft QDA. Imported documents appeared in the Documents and Categories window. Each imported document was given a document title based on the name of the file from which it was imported. Once the documents were imported from a file, he double-clicked its name in the Documents and Categories window to open the file, and read and marked for the themes and sub-themes it contained. To code the document, the researcher selected the theme from within the category tree in the Documents and Categories. The name of the theme appeared in the document window, next to the Mark button. He clicked the mouse in the text box within the document window, to the left of the Mark. He typed the first few letters of the name of the theme or sub-theme he wanted to code the document with. He pressed 'Enter' to get a list of matching themes or sub-themes. The first match showed in the space where he was typing; other matching themes or sub-themes (where applicable), were selected from the drop-down menu that appeared. 
Create a new theme by selecting Add Category from the

Project menu. Continue this process until the appropriate

number of themes has been created.

Go to the category tree at the bottom of the Documents \&

Categories window. Here all of the themes in your project are

arranged in a tree.

Select the existing theme under which you want to add a

new sub-theme by highlighting it in the tree. If no theme is

selected, the new category will be added to the base of the

tree of categories as a theme, not sub-theme.

Click the New button below the category tree. A small

window will appear prompting you for a name for the new

sub-theme. Enter the name here - this should be a short but

descriptive name for the category.

\section{Figure 3.2 Creating themes and sub-themes in Weft QDA version 1.0.1 software}

Once the researcher chose a sub-theme, he selected the part of the transcript that he wanted to code by highlighting the relevant text in the document window. To highlight the relevant text, the researcher clicked the mouse at the beginning of the text, held the mouse down and dragged the cursor to the end of the relevant text. He then clicked the Mark button at the bottom of the document window to mark the text as relating to the selected sub-theme. Texts already marked were shown in a highlighted colour on the Windows XP computer. After marking all relevant data to corresponding themes and sub-themes, the researcher reviewed all the document sections coded by themes and sub-theme side-by-side to ensure they made meaning. This also allowed the researcher to compare them, seeing what they had in common, and how they differed (see Figure 3.3). Where necessary to remove any marking already selected, the researcher selected the relevant text and clicked the Unmark button. 


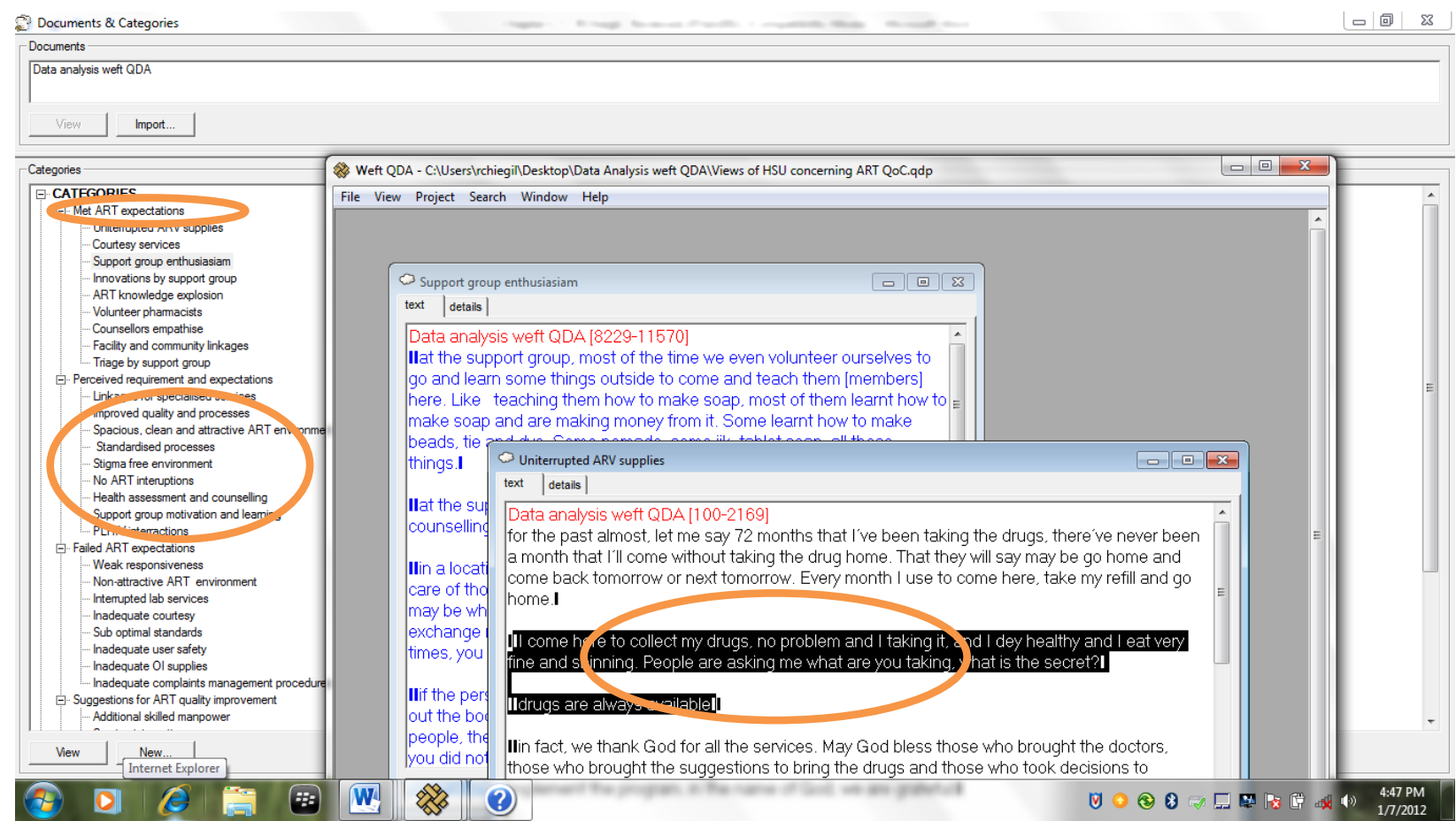

Figure 3.3 Screenshot showing themes (top left), sub-themes (lower left) and marked texts (lower central) during data analysis

As the analysis developed and the number of sub-themes increased, the researcher found that he needed to re-arrange and keep the category tree manageable. He also reviewed and ensured that sub-themes were consistently kept in tune with applicable themes. Where a sub-theme was found to be more appropriate to a different theme, he dragged the sub-theme from its current position (under theme $X$ ) in the category tree to a new and most appropriate position (under theme $Y$ ), along with all the texts that are associated to it.

Following completion of analysis of the data, the researcher exported each sub-theme in HTML format, to a file containing all its text along with its memo. To do this, the researcher ensured the sub-theme is open in the topmost, active window in the workarea. Then he chose Export from the Project menu. The screenshot on Figure 3.4 shows outcome of this action. 


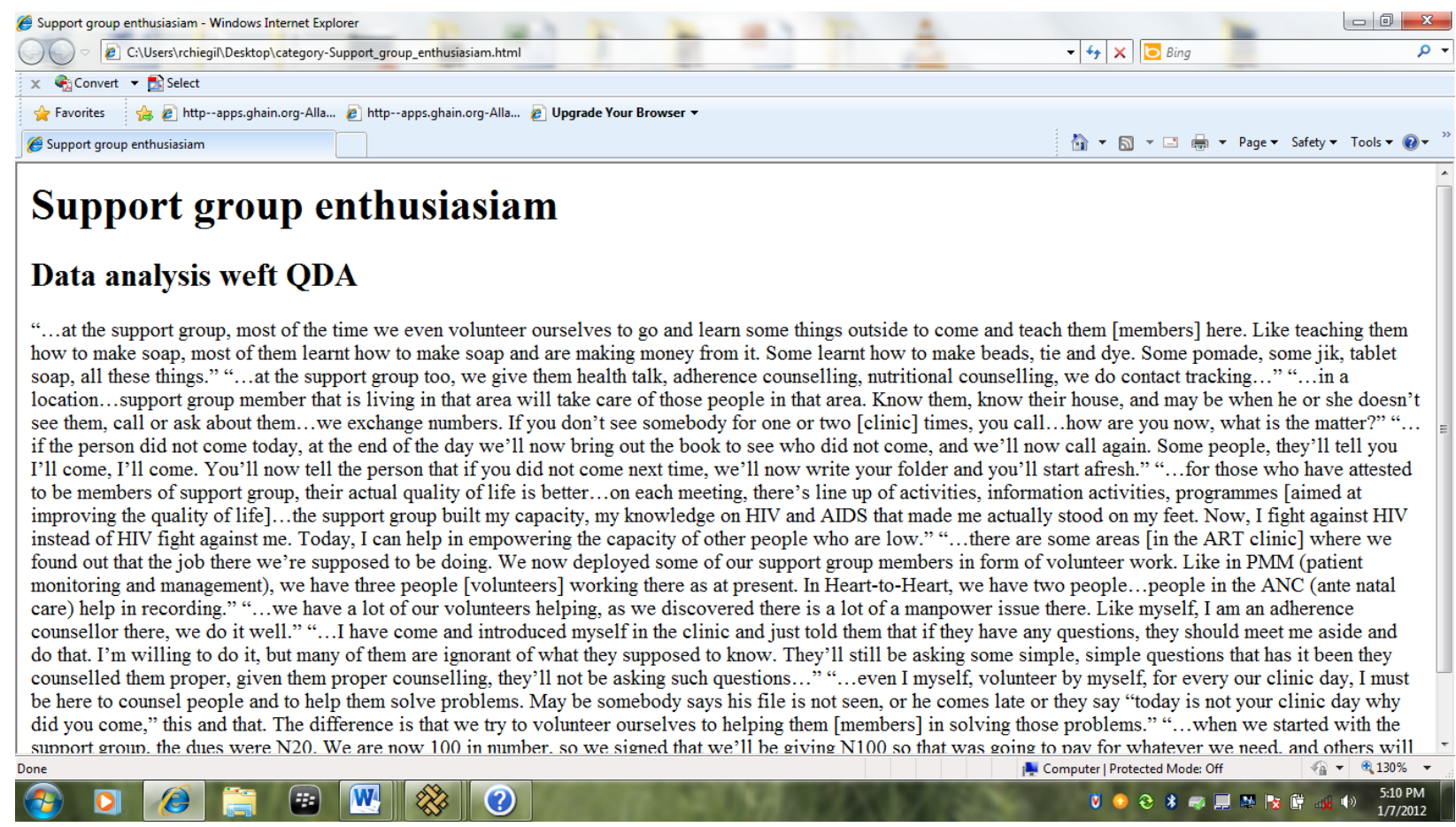

Figure 3.4 Screenshot showing exported analysed data from Weft QDA version 1.0.1 software, in HTML format

Mapping and interpretation. The researcher compared the themes and sub-themes generated from both data analysis using the framework approach and Weft QDA version 1.0.1 software, to harmonise findings. Overall, only findings which stemmed from both manual and Weft QDA version 1.0.1 software data analysis methods were listed as findings for this study. The researcher used the themes and sub-themes that emanated from both the framework approach and Weft QDA software to define concepts, map the range and nature of phenomena, create typologies and find associations between themes with a view to providing explanations for the findings.

The process of mapping and interpretation influenced by the original research objectives as well as by themes that have emerged from the data themselves. Each penultimate theme was described in detail in the research results section. The researcher provided "thick" descriptions of defining attributes, descriptions of what the end users did and did not discuss in the focus groups, and quotes from the end users that were illustrative of the themes. The researcher used multiple data sources to examine and validate conclusions about meanings. 
In the discussion section of the research report, the researcher provided commentary about the specific new knowledge generated by the study; including the end users' requirement and expectations for quality of ART in Nigerian public health facilities. The researcher also discussed specific implications of the findings to public health, tying the implications to existing literature, to enhance the credibility of the interpretations of the findings.

\subsection{TRUSTWORTHINESS OF THE STUDY}

Mdondolo, De Villers and Ehlers (2003:91) elucidate trustworthiness as the extent to which a research study is worth paying attention to, worth taking note of, and the extent to which others are convinced that the findings are to be trusted. The basic question addressed by the notion of trustworthiness, according to Lincoln and Guba (1985:290), is simple: "How can an inquirer persuade his or her audiences that the research findings of an inquiry are worth paying attention to?" When judging qualitative work, Stommel and Wills (2004:288) opine that replicability is a key standard or convention, and as a criterion for data quality, means that the readers of a research report should expect to see enough information about the data collection methods and study design so that these methods could be used again (replicated) in a similar study. Thus, to ensure replicability of this study, a descriptive study design and FGD method of data collection were clearly explained and in detail. Lincoln and Guba (1985:300) have identified one alternative set of criteria that correspond to those typically employed to judge quantitative work. They are; credibility, transferability, dependability and confirmability.

\subsubsection{Credibility}

Credibility standards involve performing specific activities that increase the trustworthiness of the reported findings (Stommel \& Wills 2004:289). It can be enhanced through triangulation of data. Other techniques for addressing credibility include making segments of the raw data available for others to analyse (peer debriefing), prolonged engagement or researcher's substantial immersion in the research process and the use of "member checks," in which respondents are asked to corroborate findings (Lincoln \& Guba 1985:313- 316; Stommel \& Wills 2004:289). 
In this study, the researcher truly engaged with the research, established valid and meaningful relationships with the participants, and was open to the deeper meanings that unfolded during the research process. The researcher also interacted with experienced research colleagues in the workplace who provided guidance for research design, data collection, and data analysis for review and consensus on how to proceed. Following the FGD and their transcription, the researcher shared the transcripts with the respondents for review and correction of the researcher's interpretation of the meaning of the data. The researcher conducted data analysis using both the manual method (framework approach) and the Weft QDA version 1.0.1 software. Results of the study were triangulated and also compared with other data sources to examine and validate conclusions about meanings.

\subsubsection{Transferability}

Transferability, according to Stommel and Wills (2004:288-289) is conceptually similar to generalisability (external validity) in quantitative studies, which refers to the extent to which findings can be generalised to other situations and target populations. Lincoln and Guba $(1985: 110-111,124)$ admit that generalisability is "an appealing concept," because it allows a semblance of prediction and control over situations. Yet they suggest that the existence of local conditions "makes it impossible to generalise." Stommel and Wills (2004:289) recommends "thick description," a very detailed description of the nature of the participants, their reported experiences, and the researcher's observations during the study. This is to provide sufficiently detailed information on the study, such that interested others could gauge the extent to which the findings might apply in another population or setting.

To meet the criteria for transferability, the report of this study provided discussions of the nature of the participants, their reported experiences/data obtained, researcher's observations during the study, methods of data analysis and interpretation of the research findings. The researcher also made references to the raw data, kept available for any interested person (researchers) to cross check or verify. This detailed information therefore, potentially renders opportunities to interested others to gauge the extent to which the study findings could be generaliseable or transferable. 


\subsubsection{Dependability}

Lincoln and Guba (1985:317) propose one measure which might enhance the dependability of qualitative research. That is the use of an "inquiry audit," in which reviewers examine both the process and the product of the research for consistency. Stommel and Wills (2004:288) on the other hand, elucidate that dependability is most conceptually similar to the concept of test-retest and internal consistency reliability in quantitative research approaches, and refers to how stable or unstable the data patterns tend to be over time or occasions.

To meet the criteria for dependability, the researcher engaged a consultant to do an independent data collection and analysis in one of the study sites. The data and analyses were then checked for comparability and similarity. Discrepancies were resolved through 'member checking' with the participants.

\subsubsection{Confirmability}

Confirmability, according to Lincoln and Guba (1985:320-321), refer to the degree to which the researcher can demonstrate the neutrality of the research interpretations, through a "confirmability audit" (Stommel \& Wills, 2004:288, calls it "audit trails.") This means providing an audit trail consisting of 1 ) raw data; 2) analysis notes; 3) reconstruction and synthesis products; 4) process notes; 5) personal notes; and 6) preliminary developmental information.

To meet the criteria for confirmability, the researcher used audit trails, in which approaches to data collection, decisions about what data to collect, and decisions about the interpretation of data were carefully documented, so that another knowledgeable researcher can arrive at the same conclusions about the data and for the protection of human subjects, as required by institutional review boards.

\subsection{REFLEXIVITY}

According to Wikipedia (2012), reflexivity refers to circular relationships beween cause and effect. This study was a qualitative study that did not indicate any relationships between variables. The study of perception of end user satisfaction with ART service 
provision was a subjective study which did not have relationships between cause and effect. However, the researcher became aware of all biasis in order to free himself from them and to aspire to get participant responses that were devoid of biasis. Reflexivity was in this study part of a solution and not a problem. In this study reflexivity referred to those act of biasis which affected the ability to obtain trustworthy responses from end users on their perception of quality, or satisfaction with antiretroviral therapy service provision in public health facilities in Nigeria.

Chow et al (2009:440) reveal that several biases based on psychosocial theories may influence end user perceptions. The cognitive consistency theory implies that an end user is more likely to respond positively to justify their own time and effort spent obtaining treatment (Chow et al 2009:440). As this was a qualitative study, the researcher took time to explain to participants, the purpose of the study and the usefulness of obtaining their true views regarding quality of services received in the public health facility. The researcher explained that the study focused on their own perceptions from which guidelines would be developed to improve quality of care. This explanation served as motivation for study participants to provide information with as minimal biasis as possible.

The second bias noted by the researcher was the Hawthorne effect that is more related to quantitative studies. According to Chow et al (2009:440), the Hawthorne effect implies that the very action of exploring the perceptions on satisfaction could increase the apparent concern relating to the delivery of health care. In this qualitative study, the researcher conducted FGDs in 6 different locations, within participants' regular settings. Each FGD was composed of 8-12 participants. Similarly, the principle of data saturation was followed in conducting FGDs.

Thirdly, Chow et al (2009:440) identified social desirability as a possible bias in end user satisfaction studies. Social desirability bias indicates that end users give positive responses because they feel that these are more acceptable to satisfy researchers (Chow et al 2009:440). To reduce social desirability biasis, the researcher explained to participants in each group the importance of the study. Data emerged from the perceptions of the participants on ART services provision in public health facilities in Nigeria. 
Fourthly, Chow et al (2009:440) identified sycophancy or ingratiating responses as a possible bias in end user satisfaction studies. Ingratiating responses bias implies that end users could give positive responses. Similarly, participants may be reluctant to provide negative perceptions in fear of prejudice from the researcher (Chow et al 2009:440).

This was a qualitative study in which the researcher used probing and focused on the principles of trustworthiness. The researcher further minimised this type of bias by assuring participants of confidentiality of anything said in the FGD. Adding that what ever information they provide will not be shared with anyone in the context that someone will be able to link to whom ever provided the information. The researcher, however, assured participants that in case they do not feel comfortable revealing their "true" perceptions in a group context, especially if they are concerned that others in the group may share their opinions, they should feel free not to state it.

Fifthly, Chow et al (2009:441) noted that self-interest bias could cause that participants (end users) provide positive responses, because they believe this will allow the health care programme to continue running, which is in their best interest. Gratitude is also a common factor (Chow et al 2009:441). The researcher explained the objectives of the study to the participants. Thus, they were encouraged to share their perceptions freely, in order to obtain trustworthy information.

\subsection{CONCLUSION}

This chapter explicated the qualitative paradigm that was used in this study, whereby an exploratory, descriptive and contextual design was used. A focus group discussion was held in each of the six participating ART sites (Anambra, Edo, Sokoto, and Yobe states, and the FCT) representing each of the six geopolitical zones of Nigeria. The study sample comprised 64 end users attending ART in the participating ART sites. The study sample was selected using a convenience sampling technique because it enabled the selection of most readily available end users for the study. Unstructured focus group interviews were conducted in this study in order to obtain albeit in an artificial setting thick, rich and detailed data that are 'unfiltered' personal perceptions of their experiences concerning quality of ART. Data was analysed using the framework approach, composed of the following stages of analysis: familiarization, identifying a 
thematic framework, indexing, charting, mapping and interpretation. To increase the credibility of the research findings, the data analysis was repeated using the Weft QDA Version 1.0.1 software for qualitative data analysis. Furthermore this chapter described measures employed by the researcher to ensure strict compliance with ethical standards relevant to protecting the rights of the participants, including nondiscrimination, beneficence, respect for persons, and justice. The rights of the institutions where data were collected was also protected through legality, integrity, openness, respect for intellectual property, confidentiality, responsible mentoring, honesty, objectivity, carefulness, responsible publication, respect for colleagues, social responsibility and competence.

Lastly, the trustworthiness of the study was addressed through explaining measures of ensuring credibility, transferability, dependability and confirmability of the study. The researcher was reflexively aware of biases to free herself/himself from them (bracket) and aspire to get participant responses that are devoid of biases.

The next chapter (chapter 4) will focus on analysis and presentation of data obtained from the six FGDs. 


\section{CHAPTER 4}

\section{DATA ANALYSIS, PRESENTATION AND INTERPRETATION OF FINDINGS}

\section{$4.1 \quad$ INTRODUCTION}

In the previous chapter, the research design and methods were discussed. This chapter focuses on data analysis and interpretation of findings. The analysis of qualitative data began in the field, during data collection, using the unstructured focus group discussion. Pope et al (2000:114) noted that this sequential analysis or interim analysis has the advantage of allowing the researcher to refine questions on the spot and pursue emerging avenues of inquiry in further depth. Data gathered were analysed on the spot to shape the on-going data collection process. Once the researcher left the field, textual data exploration continued through content analysis.

This chapter also focuses on the techniques employed during data analysis and presentation of findings obtained from the study.

\subsection{OVERVIEW OF DATA ANALYSIS}

Data analysis was conducted on all data that was gathered in the study which included observations of participants' verbal cues.

\subsubsection{Organisation of data}

The researcher spent one week listening to tapes and reading field notes to familiarise himself with the data recorded on Livescribe Pen, before data transcription began. Once he was familiar with the data, he commenced transcription onto Liverscribe workbook. The transcribed data on the Livescribe workbook, which is sensitive to the Livescribe Pen enabled the researcher to play the records over and over again, until he was convinced that the data was correctly transcribed onto the workbook. The transcribed data on the Livescribe workbook mirrors what is stored in the Livescribe Pen. Therefore, 
the researcher uploaded the transcribed data from the Livescribe Pen onto Livescribe Desktop, to enable printing and electronic data storage. The researcher printed the electronic data and used pencil and different colours of highlighters to indicate codes and highlights on concepts and attributes that were similar. The researcher coded every script as a way forward for developing a comprehensive framework for analysis. The comprehensive framework was then used for more detailed coding and thematic content analysis using manual methods. The researcher analysed all the scripts (transcripts, observational and field notes) manually to get an indication of the codes and content of the analysis. He then reviewed the codes and thematic content several times to gain mastery. From an initial number of seven themes, he continued to analyse content, merging relevant concepts and attributes, until the themes were reduced to four. Data analysis focused on exploring the perceptions of end users concerning quality of ART in Nigerian public health facilities.

\subsubsection{Reporting on techniques used for analysing the data}

As it was discussed in the data analysis methods in chapter 3, thematic content analysis was employed for data analysis. This comprised of five essential steps, viz, familiarisation, identifying a thematic framework, indexing, charting and, mapping and interpretation. Each step has been elucidated below (Pope, Ziebland \& Mays 2000:116):

Familiarisation. The researcher engaged in in-depth familiarisation of the data, in order to systematically code and carry out thematic content analysis on the data. To achieve this, the researcher was substantially engaged in immersion of the raw data by listening to tapes, reading transcripts, studying notes and so on, in order to list key ideas and recurrent themes. The researcher also built upon existing relationship with the participants to strengthen more valid and meaningful rapport. Through this process, the researcher got back to some of the participants where he had doubts regarding what was transcribed, to obtain additional clarification. Thus, where applicable, the transcript was returned to the participants to review, add to, or revise their responses. They were also encouraged to correct the researcher's interpretation of the meaning of the data, where applicable. The researcher also shared the transcript with two experienced research colleagues in his workplace to review the entire process, proffer suggestions and reach consensus on the best way to improve the data analysis. Suggestions obtained from the colleagues were used to enrich the data analysis process. This was 
done with all dedication, until full familiarity was achieved and the key themes identified. The researcher also shared the transcript for one of the focus group discussions with another experienced qualitative researcher (who served as a note taker on the field) to do an independent analysis. The analysis was then checked for comparability and similarity, and discrepancies were resolved.

Identifying a thematic framework. The researcher identified key ideas, issues, concepts, and themes as examined and referenced in the data. This was guided by the aims and objectives of the study. It was also guided by issues raised by the participants themselves, as well as perceptions or experiences that recur in the data. The end product of this stage was a detailed index of the data, labelled into manageable chunks for subsequent retrieval and exploration.

Indexing. The researcher applied the thematic framework or index systematically to all the data in textual form. He did this by annotating the transcripts with numerical codes from the index. Each index was supported by short text descriptors to elaborate the index heading. Single passages of text were made to encompass a large number of different themes, each of which was recorded in the margin of the transcript.

Charting. The researcher rearranged the data according to the appropriate part of the thematic framework to which they relate, forming charts. The charts contained distilled summaries of perceptions and experiences of end users concerning quality of ART in public health facilities. Thus, the charting process involved a considerable amount of abstraction and synthesis.

Mapping and interpretation. The researcher used the charts to define concepts, map the range and nature of phenomena. He also used the charts to create typologies and find associations between themes, with a view to providing explanations for the findings. The process of mapping and interpretation was influenced by the original research objectives, the theoretical framework (Wagner's chronic care model) as well as by themes that have emerged from the data themselves. Each penultimate theme was described and discussed in detail in the research discussions section (Section 5.2) of this thesis. 
Through the five stages of data analysis in the framework approach described above and the Weft QDA Version 1.0.1 software, the following four themes emerged and were compiled:

THEME 1: ART services that met participants' requirements

THEME 2: Participants' perceived successes in ART service provision

THEME 3: ART services that disappointed participants from meeting their requirements (dissatisfaction)

THEME 4: Participants' suggestions to improve quality of ART services

These four themes and their sub-themes are graphically illustrated in Figure 4.1 while the actual themes and sub-themes that emerged from the study are presented in Table 4.1. 


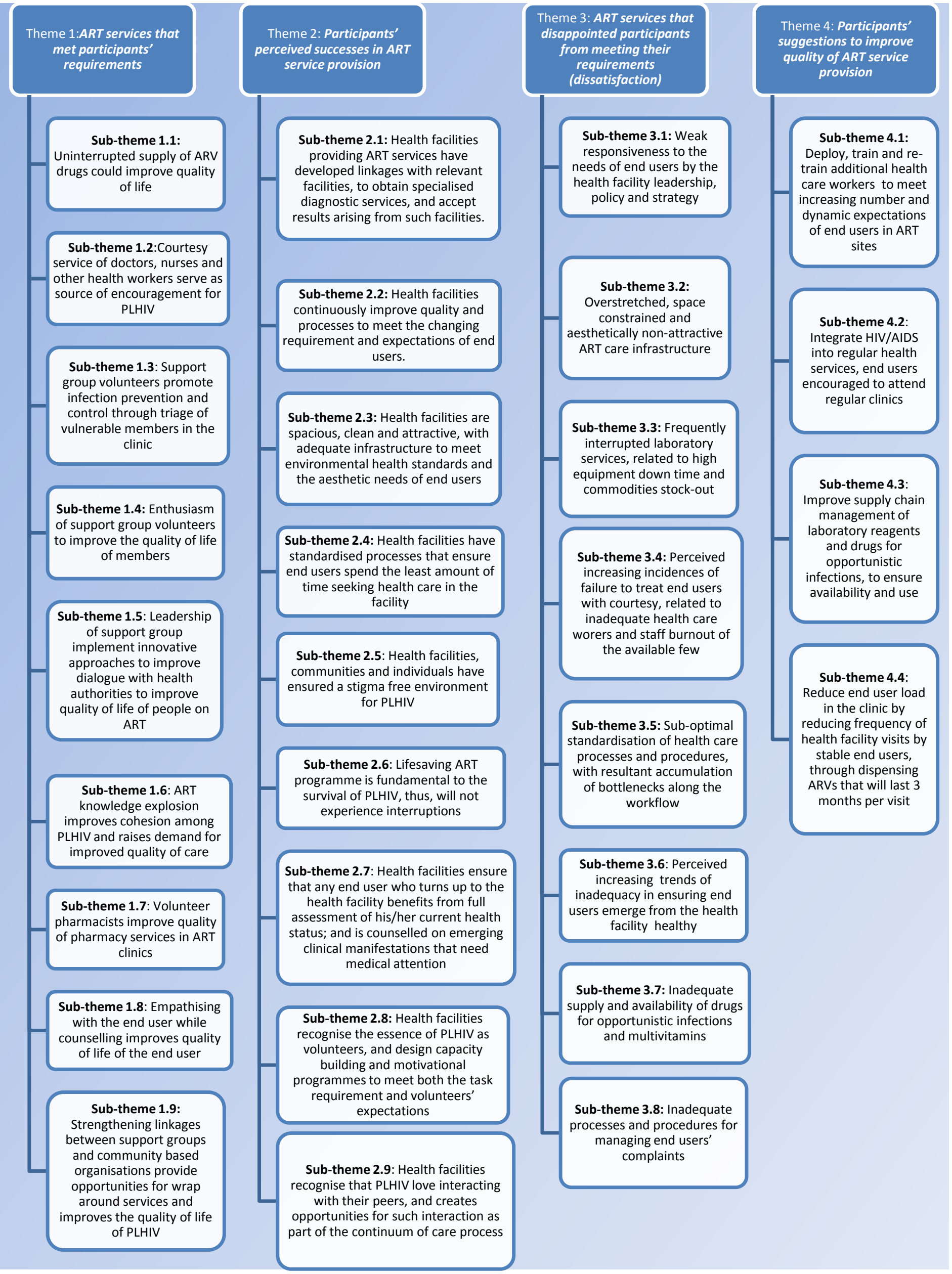

Figure 4.1 Graphic hierarchical representation of themes and sub-themes as used in this study 


\section{Table 4.1 Overview of the themes and the sub-themes}

\begin{tabular}{|c|c|}
\hline \multicolumn{2}{|r|}{ THEMES AND SUB-THEMES } \\
\hline Theme 1 & ART services that met participants' requirements \\
\hline Sub-theme 1.1 & Uninterrupted supply of ARV drugs could improve quality of life \\
\hline Sub-theme 1.2 & Courtesy service of doctors, nurses and other health workers serve as source of encouragement for PLHIV \\
\hline Sub-theme 1.3 & Support group volunteers promote infection prevention and control through triage of vulnerable members in the clinic \\
\hline Sub-theme 1.4 & Enthusiasm of support group volunteers to improve the quality of life of members \\
\hline Sub-theme 1.5 & Leadership of support group implement innovative approaches to improve dialogue with health authorities to improve quality of life of people on ART \\
\hline Sub-theme 1.6 & ART knowledge explosion improves cohesion among PLHIV and raises demand for improved quality of care \\
\hline Sub-theme 1.7 & Volunteer pharmacists improve quality of pharmacy services in ART clinics \\
\hline Sub-theme 1.8 & Empathising with the end user while counselling improves quality of life of the end user \\
\hline Sub-theme 1.9 & $\begin{array}{l}\text { Strengthening linkages between support groups and community based organisations provide opportunities for wrap around services and improves the quality of } \\
\text { life of PLHIV }\end{array}$ \\
\hline Theme 2 & Participants' perceived successes in ART service provision \\
\hline Sub-theme 2.1 & $\begin{array}{l}\text { Health facilities providing ART services have developed linkages with relevant facilities, to obtain specialised diagnostic services, and accept results arising from } \\
\text { such facilities. }\end{array}$ \\
\hline Sub-theme 2.2 & Health facilities continuously improve quality and processes to meet the changing requirement and expectations of end users. \\
\hline Sub-theme 2.3 & Health facilities are spacious, clean and attractive, with adequate infrastructure to meet environmental health standards and the aesthetic needs of eno \\
\hline Sub-theme 2.4 & Health facilities have standardised processes that ensure end users spend the least amount of time seeking health care in the facility \\
\hline Sub-theme 2.5 & Health facilities, communities and individuals have ensured a stigma free environment for PLHIV. \\
\hline Sub-theme 2.6 & Lifesaving ART programme is fundamental to the survival of PLHIV, thus, will not experience interruptions. \\
\hline Sub-theme 2.7 & $\begin{array}{l}\text { Health facilities ensure that any end user who turns up to the health facility benefits from full assessment of his/her current health status; and is counselled on } \\
\text { emerging clinical manifestations that need medical attention. }\end{array}$ \\
\hline Sub-theme 2.8 & $\begin{array}{l}\text { Health facilities recognise the essence of PLHIV as volunteers, and design capacity building and motivational programmes to meet both the task requirement and } \\
\text { volunteers' expectations. }\end{array}$ \\
\hline Sub-theme 2.9 & Health facilities recognise that PLHIV love interacting with their peers, and create opportunities for such interaction as part of the continuum of care process. \\
\hline Theme 3 & ART services that disappointed participants from meeting their requirements (dissatisfaction) \\
\hline Sub-theme 3.1 & Weak responsiveness to the needs of end users by the health facility leadership, policy and strategy \\
\hline Sub-theme 3.2 & Overstretched, space constrained and aesthetically non-attractive ART care infrastructure \\
\hline Sub-theme 3.3 & Frequently interrupted laboratory services, related to high equipment down time and commodities stock-out \\
\hline Sub-theme 3.4 & Perceived increasing incidences of failure to treat end users with courtesy, related to inadequate health workers and staff burnout of the a \\
\hline Sub-theme 3.5 & Sub-optimal standardisation of health care processes and procedures, with resultant accumulation of bottlenecks along the workflow \\
\hline Sub-theme 3.6 & Perceived increasing trends of inadequacy in ensuring end users emerge from the health facility healthy \\
\hline Sub-theme 3.7 & Inadequate supply and availability of drugs for opportunistic infections and multivitamins \\
\hline Sub-theme 3.8 & Inadequate processes and procedures for managing end users' complaints \\
\hline Theme 4 & Participants' suggestions to improve quality of ART service provision \\
\hline Sub-theme 4.1 & Deploy, train and re-train additional health care workers to meet increasing number and dynamic expectations of end users in ART sites \\
\hline Sub-theme 4.2 & Integrate HIVIAIDS into regular health services, end users encouraged to attend regular clinics \\
\hline Sub-theme 4.3 & rove supply chain management of laboratory reagents and drugs for opportunistic infections, to ensure availability and use \\
\hline Sub-theme 4.4 & id in the clinic by reducing frequency of health facility visits by stable end users, through dispensing ARVs that will \\
\hline
\end{tabular}




\subsection{DATA ANALYSIS}

In Section 4.4.1 the demographic data of participants who participated in focus group discussions were presented and quantified.This might be important for contextualising some qualitative data presented and discussed in Sections 4.4 and 5, respectively. The number and depth found in the quotes under the themes indicated data saturation.

\subsubsection{Demographic data of participants}

A brief demographic profile of participants per study site who participated in FGD was explored and the results are presented in Table 4.2. 
Table 4.2 Demographic data of FGD participants $(n=64)$

\begin{tabular}{|c|c|c|c|c|c|c|c|c|}
\hline \multirow[b]{2}{*}{ Category } & \multicolumn{7}{|c|}{ Frequency per site } & \multirow[b]{2}{*}{ Percentage } \\
\hline & NE & NW & NC & SE & sw & SS & Total & \\
\hline \multicolumn{9}{|l|}{ Gender } \\
\hline Male & 4 & 4 & 4 & 2 & 1 & 3 & 18 & $28.1 \%$ \\
\hline Female & 8 & 8 & 4 & 8 & 9 & 9 & 46 & $71.8 \%$ \\
\hline Total & 12 & 12 & 8 & 10 & 10 & 12 & 64 & $100.0 \%$ \\
\hline \multicolumn{9}{|l|}{ Age } \\
\hline Less than 20 years & 0 & 0 & 0 & 0 & 0 & 0 & 0 & $0 \%$ \\
\hline $20-30$ years & 4 & 5 & 1 & 1 & 3 & 1 & 15 & $23.4 \%$ \\
\hline $31-40$ years & 8 & 4 & 5 & 5 & 6 & 5 & 33 & $51.5 \%$ \\
\hline 41 years and above & 0 & 3 & 2 & 4 & 1 & 6 & 16 & $25.0 \%$ \\
\hline Total & 12 & 12 & 8 & 10 & 10 & 12 & 64 & $100.0 \%$ \\
\hline \multicolumn{9}{|l|}{ Occupation } \\
\hline $\begin{array}{r}\text { Applicant (just finished } \\
\text { school and actively seeking } \\
\text { to be employed) }\end{array}$ & 0 & 0 & 2 & 1 & 3 & 1 & 7 & $10.9 \%$ \\
\hline Student & 0 & 2 & 0 & 1 & 0 & 0 & 3 & $4.6 \%$ \\
\hline Civil servant & 4 & 2 & 2 & 1 & 1 & 3 & 13 & $20.3 \%$ \\
\hline Own Business/self employed & 1 & 3 & 1 & 5 & 3 & 8 & 21 & $32.8 \%$ \\
\hline $\begin{array}{r}\text { Other (unemployed or lost } \\
\text { own job, and making no } \\
\text { efforts to be usefully } \\
\text { employed) }\end{array}$ & 7 & 5 & 3 & 2 & 3 & 0 & 20 & $31.25 \%$ \\
\hline Total & 12 & 12 & 8 & 10 & 10 & 12 & 64 & $100.0 \%$ \\
\hline \multicolumn{9}{|l|}{ Highest Level of Education } \\
\hline Primary level & 1 & 3 & 1 & 7 & 2 & 4 & 18 & $28.1 \%$ \\
\hline O' level & 6 & 1 & 2 & 0 & 4 & 6 & 19 & $29.6 \%$ \\
\hline Diploma level & 1 & 2 & 5 & 1 & 1 & 2 & 12 & $18.7 \%$ \\
\hline University degree & 0 & 1 & 0 & 1 & 0 & 0 & 2 & $3.1 \%$ \\
\hline Other & 4 & 5 & 0 & 1 & 3 & 0 & 13 & $20.3 \%$ \\
\hline Total & 12 & 12 & 8 & 10 & 10 & 12 & 64 & $100.0 \%$ \\
\hline \multicolumn{9}{|l|}{ Duration on ART } \\
\hline 6 months to 1 year & 3 & 3 & 0 & 2 & 1 & 0 & 9 & $14.0 \%$ \\
\hline $2-3$ years & 3 & 7 & 5 & 4 & 5 & 8 & 32 & $50.0 \%$ \\
\hline 4-5 years & 4 & 2 & 2 & 3 & 2 & 2 & 15 & $23.4 \%$ \\
\hline More than 5 years & 2 & 0 & 1 & 1 & 2 & 2 & 8 & $12.5 \%$ \\
\hline Total & 12 & 12 & 8 & 10 & 10 & 12 & 64 & $100.0 \%$ \\
\hline
\end{tabular}


A total of 64 participants were interviewed in six focus group discussions held in six geopolitical zones of Nigeria. Their demographic characteristics are illustrated in Table 4.2. The results showed that majority of the participants $(n=46 ; 71.88 \%)$ were female, compared to $(n=18) 28.13 \%$ male participants. Most participants owned their own businesses $(n=21 ; 32.81 \%)$, compared to $(n=20 ; 31.25 \%)$ whose occupation was categorised as 'Other,' (composed of participants who stated that they do nothing for a living or lost their jobs to ill health). Most participants were within the age range of 3140 years $(n=33 ; 51.56 \%)$, compared to the least age range of $20-30$ years $(n=15$; 23.44\%). Most participants had O' level qualifications ( $n=19 ; 29.69 \%)$ and attended ART programme for $2-3$ years $(n=32 ; 50)$. It emerged from this analysis that the findings of this study could reflect the opinions of low educated, self employed females of reproductive age, who attended ART services for more than 2 years.

\subsection{DATA STRUCTURE}

Four major themes emerged from the analysis of qualitative data, as illustrated in Figure 4.2 below:

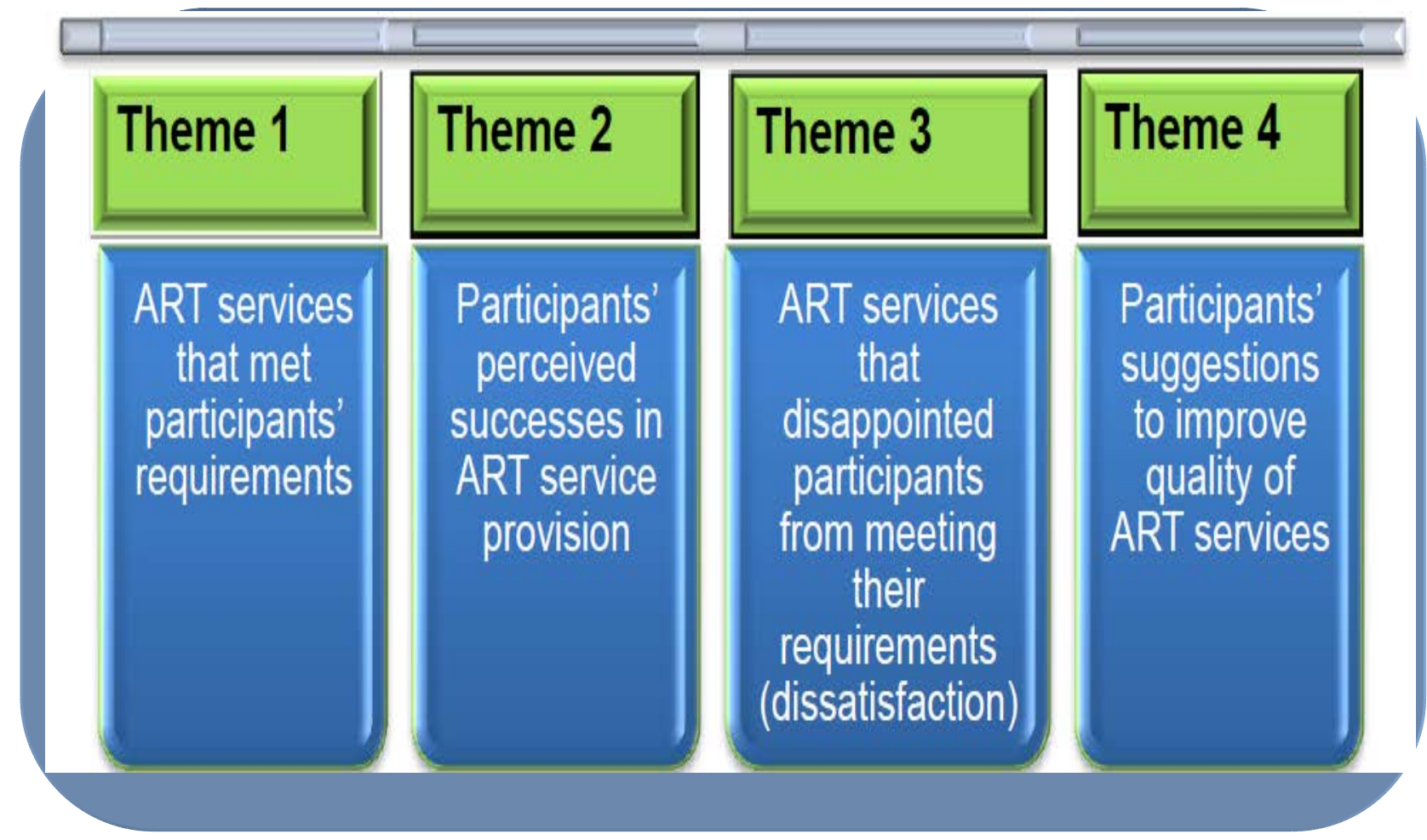

Figure 4.2 Themes that emerged from the study 
The sub-themes that emerged from the analysis of qualitative data are presented against each theme on Table 4.1.

\subsubsection{Presentation of themes and sub-themes}

The results of this study are presented along the themes and the sub-themes that were derived from the analysis of qualitative data or focused group discussions. Applicable direct quotes are supplied to substantiate relevant results. Relevant literature has been used to validate the findings of the current study.

\subsubsection{Theme 1: ART services that met participants' requirements}

This theme relates to ART services which made participants satisfied with care. Studies have evidenced that when end users participate in their care, they are more likely to be satisfied with the care. Compliance with medical advice and treatment regimens is in turn, directly related to the perceived quality of service, antiretroviral therapy adherence and the resulting health outcomes (Wouters et al 2008:5; Gill \& White 2009:14). Against this background, Olupot-Olupot, Katawera, Cooper, Small, Anema and Mills (2008:1883) maintain that even among conflict affected population, regularity of ARV drugs should not be compromised. Additionally, satisfied end users are likely to take active role in their own care, continue using medical care services, and stay with a health care worker even when there are other choices (Udoh et al 2011:220).

ART services that met participants' requirements were one of the major themes that emerged during data analysis of the six focused group discussions. Nine sub-themes emanated from this theme. Figure 4.3 shows hierarchical relationships between theme1 and its 9 sub-themes. 


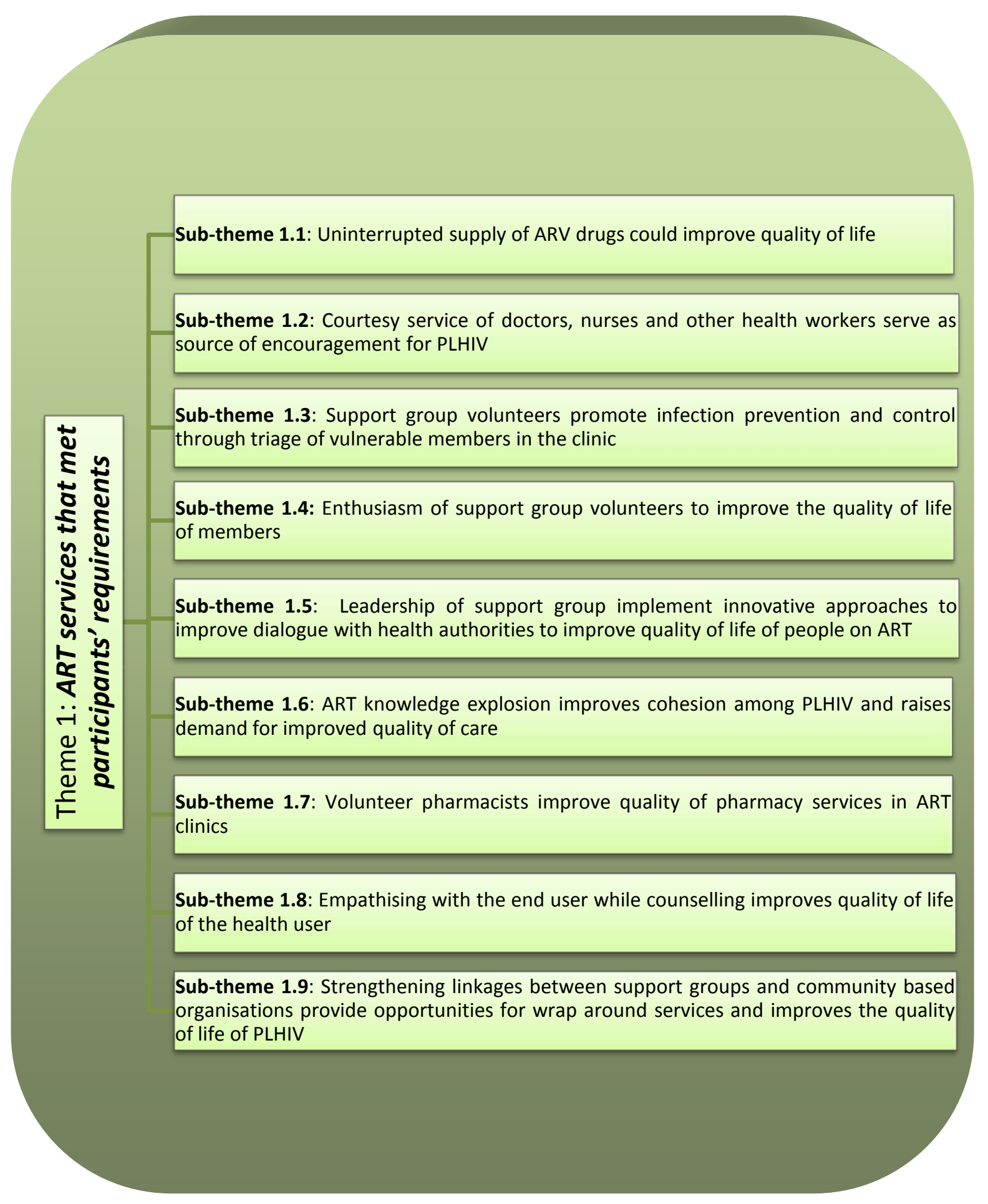

Figure 4.3 Hierarchical illustration of relationships in ART services that met end users' requirement 
The concluding statements on ART services that met participants' requirements are:

- $\quad$ uninterrupted supply of ARV drugs improves quality of life

- $\quad$ courtesy service of doctors, nurses and other health workers serve as source of encouragement for PLHIV

- $\quad$ support group volunteers promote infection prevention and control through triage of vulnerable members in the clinic

- $\quad$ enthusiasm of support group volunteers to improve the quality of life of members

- leadership of support group implement innovative approaches to improve dialogue with health authorities to improve quality of life of people on ART

- $\quad$ ART knowledge explosion improves cohesion among PLHIV and raises demand for improved quality of care

- $\quad$ volunteer pharmacists improve quality of pharmacy services in ART clinics

- $\quad$ empathising with the end user while counselling improves quality of life of the end user

- $\quad$ strengthening linkages between support groups and community based organisations provide opportunities for wrap around services and improves the quality of life of PLHIV

Users' suggestions for actions to improve the quality of ART services were:

- Uninterrupted supply of ARV drugs could improve quality of life.

- $\quad$ Courtesy service of doctors, nurses and other health workers serve as source of encouragement for PLHIV.

- $\quad$ Support group volunteers promote infection prevention and control through triage of vulnerable members in the clinic.

- $\quad$ Enthusiasm of support group volunteers to improve the quality of life of members

- Leadership of support group implement innovative approaches to improve dialogue with health authorities to improve quality of life of people on ART.

- $\quad$ ART knowledge explosion improves cohesion among PLHIV and raises demand for quality of care.

- $\quad$ Volunteer pharmacists improve quality of pharmacy services in ART clinics.

- $\quad$ Empathising with the end user while counselling improves quality of life of the end user. 
- $\quad$ Strengthening linkages between support groups and community-based organisations provide opportunities for wrap around services and improves the quality of life of PLHIV.

\section{Sub-theme 4.1.1: Uninterrupted supply of ARV drugs improve quality of life}

This refers to the security and continuous availability of ARV drugs in public health facilities. Creel et al (2002:3) assert that the end user's perspective of the quality of care emphasise availability of commodities as one of the determinants of satisfaction with care.

Participants explained during the FGD that uninterrupted supply of ARV drugs in public health facilities was one of the areas that contributed to improving their quality of life. Direct comments from the FGD participants are presented below, as evidence hereof.

The availability of ARV drugs was evident from the data:

“...for the past almost, let me say 72 months that l've been taking the drugs, there've never been a month that I'll come without taking the drug home. That they will say may be go home and come back tomorrow or next tomorrow. Every month I use to come here, take my refill and go home."

“...drugs are always available...”

A spiritual reason was given for the availability of supplies:

"...in fact, we thank God for all the services. May God bless those who brought the doctors, those who brought the suggestions to bring the drugs and those who took decisions to implement the program, in the name of God, we are grateful."

Mentally, end users were happier with themselves due to receiving supplies:

"...I am so grateful and happy with the drugs we receive from this facility. I am so happy for all the assistance."

"...then after seeing doctor, I was told that I'll be on drugs, on ART drugs. So they started giving me drugs, but that time, that's 2005, I think I bought it for two months before they started giving us the drugs free; free there. Up till now they are giving us the drugs free. It is free there up till now; they are giving us the drugs free." 
“...when I got to $[X]$ hospital, my CD4 count was $20 . .$. and in fact, when they first of all tested me in Zaria, they told my wife that this man will not survive this thing oh! But luckily when I came to $[X]$ hospital, they took me, they counselled me and I started on these drugs...immediately I was on drugs, I started seeing myself, what I could not do before I was doing it."

“....although I've not been down since l'm on drugs even before l'm on drugs. Before I'm placed on drugs is when I'm pregnant for this my second baby...even, this is my third. All of them they are negative. Even my husband is negative."

Physical implications due to the availability of supplies were mentioned:

“...the drug is very good. Like myself, before I started taking these drugs, I'll be feeling dizziness. But immediately I started taking this drug, there's nothing like dizziness again. I don't know headache and I'm a farmer, I work in the farm. So, the drug is very good."

“...I come here to collect my drugs, no problem and I taking it, and I dey healthy and I eat very fine and shinning. People are asking me what are you taking, what is the secret?"

It emerged from this analysis that end users were spiritually and physically happier due to frequent availability of drugs in the health facility.

\section{Sub-theme 4.1.2: Courtesy service of doctors, nurses and other health workers serve as source of encouragement for PLHIV}

Courtersy refers to the ability of health care workers to treat end users with politeness and affability. Courtesy treatment makes the end users feel like fellow human beings and encourage them to live positively with their HIV status. Castilo et al (2007:696) maintain that the factor with the greatest predictive power for end user satisfaction was the relationship between the end users and health care workers.

It emanated from the FGD that participants felt some doctors, nurses and other health workers treated them with courtesy, and this served as a source of encouragement to them, that they still have hope to live. Some direct comments from the FGD participants are shown below;

The collective team approach by health workers (doctors, nurses and counsellors) came to the fore as: 
“...l enjoy the treatment of doctors. The doctors, they are too good, and the nurses, the people they are working. They are too good. I enjoy the hospital because they take care of me. When I came here, I was very lean; I didn't know I'll be like this now. So, I enjoy their treatment and the drugs given to us, I enjoyed it."

"...even if you see we and doctors and nurses discussing, you'll think we are friends. We'll go as even close as talking to their ears and they are ready to listen to us."

"...all the services here is good, especially the doctors, they are very good. Their service is ok. May be you come to hospital, may be you forget something; they might encourage you to bring out all what you have been facing. Even the health workers too, whether you find your CD4 count reduced or increased, when you go there, they will ask you what your problems are? What is bordering you? All the service is good."

"...most of the doctors and what do you call them, other health workers are giving us good services."

“...the doctors are doing their best...not only doctors, even adherence counsellors; they talk to us on how we can take our drugs very well. They even mobilise other people to come and let us be together as joint group. Let us join support group so we can share our problems. If you didn't come on time or for two days you have not been seen in the hospital, they contact you and ask you what is happening, for two days you've not been coming to hospital?"

It seemed as if some health workers went the extra mile to care for clients:

“...the doctors are very friendly to us. Like myself, last month when I went to the clinic, I went there with my card and they said I should go and see doctor. I said no, I have no problem. I don't want to see the doctor. Give me my drug so that I'll go. They said no, come and see the doctor. When I went there he said I should go to do test for me. They did every test. So, if they are not friendly to us they'll not be giving us such things such as advice. Because if you just want to collect your drug and go, they'll say no, you need such testing. Test; test some things for you so that they'll know you're healthy."

“...they even tested me and said my blood pressure is high. Assuming I didn't go, I wouldn't know. They are very friendly."

“...health care workers are very understanding and cooperative. There is even a particular doctor who uses his personal money to assist any PLHIV that is in need. If you give him a call at whatever time of the day or night, he'll be there for you."

"... health care workers make frantic efforts to ensure we are well taken care of. We face challenges only when they are beyond the control of health care workers."

\section{Some participants did not have a need for interaction with health workers and for them it} was only a physical requirement to take their drugs:

"I don't think it is necessary to see a doctor [when I have no complaints]. May be, I don't have a problem why must I see a doctor? It is not too necessary. Like I do that at times. I don't have a problem I don't need to see a doctor. I want to go and just collect my drugs..." 
Other participants enjoyed a positive relationship with especially doctors:

“...as I came here in this hospital 2006 and started drinking my drugs, I never one day sick, and the doctors nice for us. And the nurses, all the people that work for us. I like their work."

“...I started my drug in 2003 so, doctors do us good."

“...just like when I started, I have met like three doctors. The one I'm seeing presently is the third one. The first one I met, that one happens to be very, very, good. I don't know his name. That was the first time I met him and I couldn't see him again. Another person took over his seat, so, that one tried bringing me closer so that I'll be able to survive the shock."

During the courtesy of health workers they encouraged clients through communication:

"...I met one doctor, who listen to my complaint, gives me a listening ear...he gives me a listening ear, very caring, listen to you, ask what your problem, whatever you tell him...in fact he is just like a brother, he listens to you very well, advice you, and when you call him, he is always available any time."

"...whenever I come there, they'll call me and some of them normally attend to me like sisters. Especially, to see doctor. When I went there last time, when I was admitted, he asked me many things. He said, how am I feeling, how the sickness, how is it disturbing me? I said I am feeling fine but this is what I feel. I feel headache that is the only one thing that is normally disturbing me. He write drug and give me and I went to the pharmacy."

"...they use to attend to us very well. They don't harass us."

"...she is one of our doctors. She use to go to our houses, asking how you are feeling and if there is anything that you are feeling; she can tackle it for you."

A spiritual connection was made between encouragement of staff and God:

"...very happy with services on-going in the hospital. May God bless the workers!"

It emanated from this analysis that team work by health care workers reinforced positive relationships and improved end users' confidence in public health faciltities. 


\section{Sub-theme 4.1.3: Support group volunteers promote infection prevention and control through triage of vulnerable members in the clinic}

Support group of PLHIV participate in volunteer work to support health care workers in end user triaging, thereby separating vulnerable end users from sources of potential health facility acquired infections. Following quality improvement initiatives in the US from 2004 to 2007, AHRQ (2010:138) reports that the rate of deaths following complications of care declined from 128.9 to 105.7 per 1,000 admissions of adults ages between 18-74. This indicates that health care complications and death can be reduced through appropriate quality improvement initiatives, which may include end user triage.

Analysis of FGD showed that participants were of the view that support group volunteers promote infection prevention and control through triage of vulnerable members in the clinic. Direct comments from the FGD participants were as follows:

Participants demonstrated understanding of the need to separate persons with highly communicable diseases from others who are vulnerable, as a means of infection prevention and control in health facilities:

\footnotetext{
"...I am a member of the IPAC [infection prevention and control committee]. So, when I go to the meeting, I come and relate anything we discussed there to the support group, even at the clinic. And let them know if they are having TB [tuberculosis], those people that are coughing we use to separate them. We let the doctor see them first, before other people, so that they can go early."

"...there is much measure [for infection prevention and control]. That is why they separated the DOTS from other departments. You know TB is airborne disease which anybody can be affected. Is not like HIV which is not airborne disease. The DOTS is far away from other areas. The Diabetes is not airborne, the hypertension is not also airborne, and that is why they now group this people with us to MOPD [medical out patient department]. But that for TB, they separated it far away."

"...those who came early should be served first. However, if someone is pressed and request to be attended to before others, such request is facilitated by the support group volunteers and implemented by the health workers."
}

It emerged from the analysis of the FGD that volunteers demonstrate awareness of the risk of infection transmission in the health facilities and participate in strategies to promote infection prevention and control through triage of volunerable members in the clinic. 


\section{Sub-theme 4.1.4: Enthusiasm of support group volunteers to improve the quality of life of members}

This relates to the passion and eagerness of support group members to be of assistance to their peers, to help them do what they could not do any longer. Wagner et al (2007:871) lament that to minimise end user dropping out of care, structures (such as support groups) should be developed to enable end users provide support to each other, and have a "safety net" for helping each other remain in care.

Participants explained during the FGD that support group members express enthusiasm to serve as volunteers to improve the quality of life of their members. Direct comments from the FGD participants as evidence hereof, include:

Time and resources are committed to learn new experiences in order to help PLHIV maintain their up keep:

“... at the support group, most of the time we even volunteer ourselves to go and learn some things outside to come and teach them [members] here. Like teaching them how to make soap, most of them learnt how to make soap and are making money from it. Some learnt how to make beads, tie and dye. Some pomade, some jik, tablet soap, all these things."

“... at the support group too, we give them health talk, adherence counselling, nutritional counselling, we do contact tracking..."

"...for those who have attested to be members of support group, their actual quality of life is better...on each meeting, there's line up of activities, information activities, programmes [aimed at improving the quality of life]...the support group built my capacity, my knowledge on HIV and AIDS that made me actually stood on my feet. Now, I fight against HIV instead of HIV fight against me. Today, I can help in empowering the capacity of other people who are low."

"....when we started with the support group, the dues were N20. We are now 100 in number, so we signed that we'll be giving N100 so that was going to pay for whatever we need, and others will be used for other things. Then, we asked everybody to sign the increase to N100."

Volunteers are delineated by geographic convenience to assist support group members living around their community:

“...in a location...support group member that is living in that area will take care of those people in that area. Know them, know their house, and may be when he or she doesn't 
see them, call or ask about them... we exchange numbers. If you don't see somebody for one or two [clinic] times, you call... how are you now, what is the matter?"

\section{Contact tracking is a key function of support group volunteers:}

“... if the person did not come today, at the end of the day we'll now bring out the book to see who did not come, and we'll now call again. Some people, they'll tell you I'll come, I'll come. You'll now tell the person that if you did not come next time, we'll now write your folder and you'll start afresh."

"...even those that are not coming we still call them on phone. Those that have no phone we go to their house."

Participants identify and fill human resource gaps in health facilities:

“...there are some areas [in the ART clinic] where we found out that the job there we're supposed to be doing. We now deployed some of our support group members in form of volunteer work. Like in PMM (patient monitoring and management), we have three people [volunteers] working there as at present. In Heart-to-Heart, we have two people... people in the ANC (ante natal care) help in recording."

"...we have a lot of our volunteers helping, as we discovered there is a lot of a manpower issue there. Like myself, I am an adherence counsellor there, we do it well."

"...I have come and introduced myself in the clinic and just told them that if they have any questions, they should meet me aside and do that. I'm willing to do it, but many of them are ignorant of what they supposed to know. They'll still be asking some simple, simple questions that has it been they counselled them proper, given them proper counselling, they'll not be asking such questions..."

"...even I myself, volunteer by myself, for every our clinic day, I must be here to counsel people and to help them solve problems. May be somebody says his file is not seen, or he comes late or they say "today is not your clinic day why did you come," this and that. The difference is that we try to volunteer ourselves to help them [members] in solving those problems."

“...volunteers should be allowed to enter the hospital admission wards and care for PLHIV, since health care workers are not able to meet all their needs."

It emanated from this analysis that there is growing enthusiasm among PLHIV to serve as volunteers. Volunteers commit time and resources to identify and fill human resource gaps in both communities and health facilities. 


\section{Sub-theme 4.1.5: Leadership of support group implements innovative approaches}

to improve dialogue with health authorities to improve quality of life of people on ART

This relates to forming advocacy teams from the leadership of the support group of PLHIV to dialogue with health authorities on issues relevant to the welfare of their members. These issues may compose of long waiting time, interruptions in commodities supply, and other aspects of poor quality service. Madeiros et al (2010:402) affirm that a relationship involving respect and health care workers really listening to their end users makes the difference in health provision.

It emerged from the FGD that participants opined that the leadership of support group consistently develop and implement innovative approaches aimed at improving dialogue with health authorities. Direct comments from the FGD participants are presented below as evidence hereof.

Leadership of support groups mediate between health authorities and their members:

"...so I know what is going on there, so I use to come down and calm them down that nobody is sitting there idle, so they should be patient, before 2 O'clock, everybody has dispatched."

"... what we now arrange is to collect the number of the man in the lab, so most of us we collect the number. So we call, we normally call to know whether there is reagent, so whenever they say there is reagent, by 5am [the following morning], people are here waiting."

“...through the Committee of the support group, I've seen some like last week...we were complaining about the shortage of manpower. We went straight to the Medical Director...he gave us a listening ear. We sat in one place there for more than 30 minutes, we were interacting. He now told us why there is a shortage here, why this shortage is not the same there. That's why the manpower is how we now need to manage this because the manpower is no longer there."

“...for me, as far as l'm concerned, some of these things boil back on understanding. Where about three persons look for folders for may be about 200 people on a clinic day. Looking for folders, they may become so tensed up ...not all of them are trained on emotional management." 
Leadership of support groups monitor implementation of resolutions/action points reached with health authorities:

"...everything that we discuss, they [health facility leadership] implement it. Like where we send out people to assist in PMM and others, it is discussion we held .... Where health facility leadership said] if we can bring some of our members to assisting because of manpower. We called our meeting and our members volunteered [to work]."

"...many of the nurses that use be ruling us bad, due to this complain all the time, many of them have changed their attitude. They have changed their attitude. Like that matron upstairs, she now behaves better, all this kind of shouting, and screaming and driving people aside. She has now come to realise that her job is also on edge because of the too much complaints on her."

"...when there is any little complains I knew about, they were able to sort the problem. One of us just said a lady who was shouting is no longer shouting."

“...we thank God because since all these strike periods we've not been having challenges because if strike period is coming and we notice it, I make sure I let [the authority] know about it...[the authority] will make sure that the doctor must come to attend to us, even during strike..."

Analysis of this FGD revealed that leadership of support groups mediate between health authorities and their members to manage complaints improve dialogue and monitor implementation of any agreed action steps.

\section{Sub-theme 4.1.6: ART knowledge explosion improves cohesion among PLHIV and raises demand for improved quality of care}

Widespread education and continued search for knowledge regarding their care raises end users' requirement and expectations for improved quality of care. Coulter (2010:4) argues that end users' attitude to the likely benefits and risks of their illness should be a key factor in ensuring an informed choice of treatment. Studies have found that end users who report greater involvement in medical care are more satisfied with their health care workers (Beach et al 2007:1119; Coulter 2010:4; Colvin et al 2003:S86).

It emanated from the FGD that participants felt that ART knowledge explosion improves cohesion among PLHIV and raises demand for quality care. See direct comments from the FGD participants below, as evidence hereof. 


\section{End users want to participate in decisions regarding their care:}

"...you people will take your own decision and carry the matter up, because anything concerning all of us, we don't live it for one person."

“...see, it is good to have meeting with us and your Coordinator, whether we can go and sell our own idea to $[Y]$ hospital."

PLHIV feel more comfortable seeking help from their peers than from anyone else:

"...like the coordinator of our meeting... he is the one, he has concern on us very well. If we have any problems, like something that we don't know, he'll say don't fear to ask questions. Ask questions so that if you ask questions, if he cannot answer us, he'll go and ask the doctor. In our meeting, the doctor doesn't come there, but we shall ask all the questions and he'll take the questions to the doctor."

\section{Cohesion grows among support group members:}

"...she came here 2006 as spinster, she got married and has gotten 2 children. She just gave birth 2 weeks ago. l've even gone to her house today, to bath her baby."

"...there was a nurse... she was tested positive for HIV and at that time, she was conscious and admitted in the hospital. But some of the nurses were showing stigma on her, despite the fact she was a nurse like them. So, I need, I think as support group members...power that they can have access to the people in the hospital. They go round in the hospital, those that are admitted and hear their problems so that that aspect of stigma will be resolved."

Participants demonstrate good understanding of requirements for their care:

"...like some of us who already have nice jobs, if you are taking these drugs you need to eat good food to enable the drug work. If you are just taking drug in the morning eat eba, in the afternoon eat eba, in the evening small gari, there's no way the drug can work."

"...we were informed that anyone of us taking ARVs should report immediately to hospital whenever we feel any abnormal sign or symptom. However, whenever we come to the hospital during odd hours of the day, we find no doctor dedicated to our care. Therefore, we need to have at any time of the day, a doctor that is well experienced and knowledgeable in ART to be on standby for us."

Increased knowledge of ART adherence influence end users to detest the notion of spiritual healing as cure for AIDS:

"...we don't allow Pastors to come and preach in our place because we have problems with the Pastors. So, this [Medical Director] now bring Pastor, so I challenged the Pastor. So even if you are coming to tell people about the word of God, is not bad but we have to train you to talk to them because our own this thing is different. May be 
when you tell somebody that, "God have healed you...you have no right to come to hospital, what are you taking drugs for, God have cured you." It is very, very dangerous to our own health. So if we meet you in this hospital we need to tell you some certain words which you'll not mention to the patients. So, [Medical Director] now said I am too stubborn. That I don't have to challenge him, that nothing concern me...that doesn't I believe God? I said I believe God but we need to train Pastors."

“...there is a traditional healer who provides drugs claimed to cure AIDS. He tells his clients to stop taking any ARV received from the hospital and take only his own. We have asked all support group members not to patronise these fake drugs. We have also informed SACA [State Agency for the Control of AIDS] to stop this man from operating in the state."

“...we have educated our support group members to adhere only to their hospital ARVs, to avoid developing resistance as a result of switching between traditional and modern medicines."

"...a couple of patients who took the traditional medicine have since lost their lives, while others are lying down very ill... will you see death coming and continue to take the traditional medicine?"

It emanated from this FGD analysis that increased knowledge and understanding is mounting among end users, characterised by increased cohesion among peers, insistence on greater quality of care, and potentials for revolution against health facilities that fail to provide needed quality of care.

\section{Sub-theme 4.1.7: Volunteer pharmacists improve quality of pharmacy services in ART clinics}

Following dearth of Pharmacists in public health facilities, community pharmacists organise themselves to provide support to health facilities in form of volunteerism. Their professional activities in some health facilities which otherwise have no Pharmacists have caused remarkable impact on quality of service. Barbosa (2011:103) reveal that to improve satisfaction with pharmaceutical care there is need for greater attention and educational activities with end users who mostly have low rates of education.

Analysis of FGD indicated that participants were of the view that volunteer pharmacists have consistently supported the health facility pharmacists to close the human resource gaps in the pharmacy, to meet their expectations for quality pharmacy services. Below are direct comments from the FGD participants as evidence hereof. 
Participants described activities of volunteer pharmacists as user focused:

"...these [volunteer pharmacists] are the people doing the work in our pharmacies."

"...actually, the pharmacists are doing the real work. They are trying, but there was a time there was only one Pharmacist, who works just there, before [the authorities] sent volunteer pharmacists to come and assist. [The Pharmacists] will first of all counsel you, show you your drugs, tell you when [to take], ask you questions how have you take it? You tell them and they'll now correct you and they'll write on the container. The one of the morning, they'll now write one in the morning, one in the evening. Then the one at night, they'll also write one at night. But the problem we are having with some of our PLHIV, they'll remove the container and throw it outside...those are the first timers. Most of the time, we go there to correct them but for the Pharmacists, they are doing their best."

It emanated from this study that participants appreciated the work of volunteer pharmacists, describing their approach to care as user focused.

\section{Sub-theme 4.1.8: Empathising with the end user while counselling improves quality of life of the end user}

A counsellor who commiserates or feels for the end user while counselling builds the end users' confidence and makes him/her feel good again. Stenson et al (2005:952) noted that end users must be properly counselled and empowered to recognise and accept quality of care. Similar findings were reported by Proctor et al (2006:377) and Kabbash et al (2010:481).

It came out from the FGD that participants opined that they feel satisfied and empowered when health workers empathise with them, while counselling. The following are direct comments from the FGD participants in support of this:

Participants expressed confidence in the quality of counselling services in the health facilities:

"...in fact, their counselling here is good. They tell you the importance of what you are about to start and tell you you shouldn't stop taking it [the ARV drug]"

"...counselling, I believe is in all the areas like the Heart-to-Heart, to PMM, from PMM to social welfare. Social welfare does the major counselling and adherence...they give you thorough counselling because that is what they are meant for. After giving you counselling, they refer some to support group, that's why we have office here. So we now use ourselves as case study to them. Those who doesn't believe that they'll be well 
after taking the ARV, we'll now let them know that some of us have been living with it for 3 years, 2 years, 4 years and are still alive. Based on this, they'll now believe."

"...that very Heart-to-Heart office, there are quality services there. They counsel you very well before they put you to test. They will ask you very well before they'll do the test. They'll ask you from ...because at times once someone tested positive, if is a woman, she'll start crying. They make sure that the person is well counselled and calm...I recommend their quality service that is in counselling..."

Participants trust the quality of health care workers providing counselling services:

"....in PMTCT...once they discover somebody positive, the Matron there personally sit the person down, counsel her and send her immediately for adherence. But first of all, let me know that for my baby to be safe I thank them for the way they advised me, my baby is safe. They are doing well. People there are not harsh; they attend to us very well."

“...doctor said I have to go and attend adherence counselling. They will tell me the dos and don'ts of the drug... The nurses there, they then told us about the drug, once you start taking the drug you don't have to stop. The drug is for life, because there is no cure. For you to continue to stay alive, you must continue taking the drug. You choose time for yourself and they will agree with you for the time you'll have to be taking your drug."

Analysis of the FGD revealed that empathising with the end users while counselling empowers end users to take responsibility for self care and improves quality of life.

\section{Sub-theme 4.1.9: Strengthening linkages between support groups and community based organisations provide opportunities for wrap around services and improves the quality of life of PLHIV}

This refers to the established channels of communication and relationships between the support groups and communities, through community based organisations. These relationships re-inforce support services for PLHIV and provide greater access to activities leading to improvement in their quality of life. Better community support for health care workers and for end users on chronic illnesses is a perennial theme in end user surveys (Coulter 2005:1200; Wagner 2007:871).

Participants explained during the FGD that strengthening linkages between support groups and community based organisations provide opportunities for wrap around services and improves the quality of life of PLHIV. Direct comments from the FGD participants are shown below, as evidence hereof. 
Linkages with community based organisations were mentioned by study participants:

"...we told them and they are helping us. They always provide us with rice sometimes."

"...there is a manual [referral directory] that contain all the organisations, all those volunteer organisations, and donor organisations...like I myself, there are people that we linked through that manual [directory] and they support us with materials."

Community pharmacies established linkages with public health facilities to support continuum of care for PLHIV:

"...there are some pharmacies, there are some hospitals who normally help us for free treatment... may be doctor write prescription to go and buy medicine. You can send the person, you give a reference note. May be the drug in that pharmacy is supposed to sell at N1000, they'll sell may be N800 or N500 to the person."

Analysis of the FGD revealed that strengthening linkages between health facilities and community based organisations reinforce continuum of care for PLHIV.

\subsubsection{Theme 2: Participants' perceived successes in ART service provision}

This theme relates to participants' health care experiences that they perceived as successes in ART service provision. Wouters et al (2008:2) and Liu and Wang (2007:266) reports that end user satisfaction has become an important performance measure and outcome of health care.

Participants' requirement and expectations for ART services was one of the major themes that emerged during data analysis. Nine sub-themes appeared to relate to this theme. Figure 4.4 shows hierarchical relationships between theme 2 and its 9 subthemes. 
Sub-theme 2.1: Health facilities providing ART services have developed linkages with relevant facilities, to obtain specialised diagnostic services, and accept results arising from such facilities.

Sub-theme 2.2: Health facilities continuously improve quality and processes to meet the changing requirement and expectations of end users.

Sub-theme 2.3: Health facilities are spacious, clean and attractive, with adequate infrastructure to meet environmental health standards and the aesthetic needs of end users

Sub-theme 2.4: Health facilities have standardised processes that ensure end users spend the least amount of time seeking health care in the facility

Sub-theme 2.5: Health facilities, communities and individuals have ensured a stigma free environment for PLHIV.

Sub-theme 2.6: Lifesaving ART programme is fundamental to the survival of PLHIV, thus, will not experience interruptions.

Sub-theme 2.7: Health facilities ensure that any end user who turns up to the health facility benefits from full assessment of his/her current health status; and is counselled on emerging clinical manifestations that need medical attention.

Sub-theme 2.8: Health facilities recognise the essence of PLHIV as volunteers, and design capacity building and motivational programmes to meet both the task requirement and volunteers' expectations.

Sub-theme 2.9: Health facilities recognise that PLHIV love interacting with their peers, and creates opportunities for such interaction as part of the continuum of care process.

Figure 4.4 Hierarchical illustrations of relationships between themes and 9 subthemes in participants' perceived successes in ART services 
The concluding statements on participants' perceived successes in ART services are that health facilities:

- $\quad$ providing ART services have developed linkages with relevant facilities, to obtain specialised diagnostic services, and accept results arising from such facilities

- continuously improve quality and processes to meet the changing requirement and expectations of end users

- are spacious, clean and attractive, with adequate infrastructure to meet environmental health standards and the aesthetic needs of end users

- $\quad$ have standardised processes that ensures health service users spend the least amount of time seeking health care in the facility

- $\quad$ communities and individuals have ensured a stigma free environment for PLHIV

- need lifesaving ART programme that is fundamental to the survival of PLHIV, thus, will not experience interruptions

- $\quad$ ensure that any end user who turns up to the health facility benefits from full assessment of his current health status; and is counselled on emerging clinical manifestations that need medical attention

- $\quad$ recognise the essence of PLHIV as volunteers, and design capacity building and motivational programmes to meet both the task requirement and volunteers' expectations

- $\quad$ recognise that PLHIV loves interacting with their peers, and creates opportunities for such interaction as part of the continuum of care process

Users' suggestions for actions to improve the quality of ART services were:

- $\quad$ Health facilities providing ART services have developed linkages with relevant facilities, to obtain specialised diagnostic services, and accept results arising from such facilities.

- $\quad$ Health facilities continuously improve quality and processes to meet the changing requirement and expectations of end users.

- Health facilities are spacious, clean and attractive, with adequate infrastructure to meet environmental health standards and the aesthetic needs of end users. 
- Health facilities has standardised processes that ensures health service users spend the least amount of time seeking health care in the facility.

- $\quad$ Government, communities and individuals have joined forces to ensure a stigma free environment for PLHIV.

- $\quad$ Lifesaving ART programme is fundamental to the survival of PLHIV, thus, will not experience interruptions.

- Health facilities ensure that any end user who turns up to the health facility benefits from full assessment of his current health status; and is counselled on emerging clinical manifestations that need medical attention.

- Health facilities recognise the essence of PLHIV as volunteers, and design capacity building and motivational programmes to meet both the task requirement and volunteers' expectations.

- Health facilities recognise that PLHIV loves interacting with their peers, and creates opportunities for such interaction as part of the continuum of care process.

\section{Sub-theme 4.2.1: Health facilities providing ART services have developed linkages with relevant facilities, to obtain specialised diagnostic services, and accept results arising from such facilities.}

This refers to identification and designation of reputable specialised diagnostic facilities where laboratory test results could be accepted by public health facilities providing ART services. Coulter (2010:2) report that end users could choose where they were referred to for laboratory tests, or choose the specialist diagnostic team that will provide their laboratory tests, if they so wish.

It emerged from the FGD that participants expect health facilities that provide ART services to have developed linkages with relevant facilities, to obtain specialised diagnostic services, and accept results arising from such facilities. Direct comments from the FGD participants are shown below, as evidence hereof.

End users perceive that public health facilities should accept lab results from every reputable lab: 
"...I had to go at Teaching Hospital to go and do the test. When I did it there and bring the result, the doctor rejected it, and said that he'll not accept the result."

"...I don't know if there's anything that somebody can do whether if they can give us form from here so we can go and do in any reputable clinic outside, if they can accept it...so not to delay the CD4 count."

"... if somebody is denied the right of being able for an opportunity to do the routine CD4 count and the person is able to get the CD4 count done in any other site, the doctor should recognise it."

Health facilities require back-up laboratory machines to avoid interruptions in lab services:

"...the CD4 count [machine] should be more than one [per site], in case one breaks down so that the other should be working."

It emerged from the analysis of the FGD that no end user should be turned back home for lack of opportunities for determining their lab results. Health facilities should develop linkages with other specialised facilities to refer end users or their samples to complete lab tests. Health facilities should also maintain back-up laboratory machines to avoid interruptions in lab services.

\section{Sub-theme 4.2.2: Health facilities continuously improve quality and processes to meet the changing requirement and expectations of end users}

This refers to constant efforts by health facilities to implement continuous quality improvement initiatives in order to meet the changing needs of end users. Findings of a study in Sao Carlos Hospital School showed that the adoption of a humanised and user focused model of assistance in health care in this hospital resulted in end user satisfaction (Ricci et al 2011:1125). A similar result was reported by Booysen et al (2007:283).

Analysis of FGD showed that participants expected health facilities to continuously improve quality and processes to meet the changing requirement and expectations of end users. Some direct comments are provided below to buttress this point:

End users demand more sophisticated type of care

"...we want them to be doing viral load for us, viral load they are not doing it here. We want them to be doing it for us." 
Improve internal processes to reduce end users' dissatisfaction with services:

"...let the pharmacist ...the doctor ...the counsellor ...just put them closer, so it should be a bigger place. Or let them appoint someone to carry the folders to the pharmacy, not the patient carrying their folders."

"... what should be done is simply let the CD4 count machine be made functionable [functional]. If it becomes functionable [functional], if it starts working well, maybe you have two or three CD4 count machines there. These things shouldn't be there; you come anytime you are scheduled to. But the issue is if it is not functioning and so people are packed up and told without your CD4 count next appointment, no drug or something of that nature. So if people, and those people [who give gratifications], I am not standing in for them but some of them accessing treatment here come from [far places] and there is no way they could make it to those CD4 count there [as early as possible]."

Institute measures to prevent extortion of end users by syndicates:

"... What I am suggesting is that to advice the person that is doing it [extorting money from patients] to stop doing it. Because they will not bribe to do it; why it is so difficult is that some people will wake up very early to make sure, but when they come here, they'll be told that their time has passed. So, my suggestion is that to approach the person that is doing it so that the person will stop doing it."

\section{End users demand automation of record keeping systems to enhance easy storage and retrieval of data:}

"...sometimes, if they didn't see your folder, maybe l'll be number one, and we are about 100 for that day. If they didn't see my folder, may be they see number 30 or others, they'll be attending to them, they'll take action before attending to me. Even, sometimes I use to go 1pm or 1.30pm because they didn't see my folder on time."

"...for the PMM, we were saying about the personnel. I know us... any folder that is not traced or any difficulties getting such. The only difficulty will be new clients. You'll just ask of the card number and click on the LAMIS (Lafiya Management Information System, an electronic medical record) to access all r e cords for existing clients."

\section{Participants demand segmentation of record retrieval terminals by age:}

“...sometimes, you may see a mother that just gave birth may be one week. May be she come to see doctor. She'll come with the baby, the new born baby. In a place where adults stay, and you don't know whether there's somebody that have TB around there. And not even that, when that person reach there that they'll even attend to that person. They'll tell her to go and wait until when it reach her turn, before they'll bring out the folder. So, I'm suggesting if they'll separate pediatric folder from the adult and be keeping it in the place where they use to see children, so that when you come for children you just go straight there. They will remove the folder and see you there..." 
Participants demand gainful employment of PLHIV:

"...there are some of our members who are not doing anything...most people the reason why they just collapse at last is they take their drugs, don't eat fine. Some cannot afford two square meals per day. So if there is anything, like there were some people who came here to rehabilitate people to have something doing. May be, if there can be anything like that."

End users demand support in form of micro-credit scheme for support group members:

"...at [another support group], they borrow them N40,000...at end of the month they pay back N2, 000. They give them 20 months to pay back everything."

Uninterrupted, affordable or free supplies of basic care kits:

"...they [PLHIV] use to come and ask that they need water guard; whether we have or not. If we don't have, we tell them we don't have. They are telling me that they use to but at N150 to N200 outside, but some of them do not have the money to buy."

"... and the issue of basic care kit, like water guard. Some use to buy, many of us cannot afford that N150 to buy."

Participants demand assistance in form of food and food supplements:

“...we need assistance in the areas of food and food supplements."

"...may be, if we can have some multivitamin drugs and antibiotics."

"...like we use to go one support group ...last Saturdays of the month. If you finish the support group they give us rice, milk, beans and immune boosters; but here they don't give us anything. That's why they [PLHIV] don't come here."

It emanated from the FGD that end users' expectations are constantly increasing in sophistication; therefore, internal processes (such as pharmacy and record keeping systems) should be continuously improved. End users should also be empowered to cope with changing socio-economic conditions, in order to retain them in care. 
Sub-theme 4.2.3: Health facilities are spacious, clean and attractive, with adequate infrastructure to meet environmental health standards and the aesthetic needs of end users

This refers to the treatment melieu for health care settings. End users perceive to have a health care setting that is admirable to any end user; that will not discourage an end user from attending health services, and that meets required standards of a health care setting. Pietrovsk and Dall'Agnol (2006:630) indicate aesthetics and cleanliness of the environment as factors that interfere with end user satisfaction. Other studies have reported similar results (Medeiros et al 2010:402, Agha \& Do 2009:87).

Analysis of FGD indicated that participants expect that health facilities are spacious, clean and attractive, with adequate infrastructure that meet environmental health standards and the aesthetic needs of end users. See below, direct comments from the FGD participants as evidence hereof.

Consistent availability of clean water supply in health facilities:

"...in terms of infrastructure, water in a hospital is very important. Because that is why like in my hospital, most of the time they lack the toilets. The patient doesn't have to go too far to urinate or to ease himself, maybe because of that water that is not flushing. They don't have enough water, so we need water."

Constant electricity supply:

“...the light is not much quality in our hospitals, because most of the time NEPA (National Electric Power Authority) disrupt attention when doctor is attending to you or when lab are trying to record something. Is just light, there are some people who are doing something they don't see without light. So, light is very good, just as water."

Adequate ventilation of health care settings:

"...because the consulting rooms, there's a window outside that receives breeze, we don't have problems about ventilation in most of the consulting rooms."

"We have problem with ventilation. Look at the way they construct the place. They didn't do it in such a way that there'll be ventilation..."

Health care setting should be comfortable for end users to feel relaxed: 
"...the [health facility] environment is not really attractive. There should be a cooling whatever, like an AC [air conditioner] ...the place is always congested, crowded, so we need that AC to make the place accommodating. ..."

Aesthetically attractive, to give a home like feeling to end users:

\begin{abstract}
“...in terms of may be making the place too beautiful, may also be a problem because there's a client that came to me. ...I was counselling this client; he was like...if this thing is a good thing, why are they making the place look so special? Just look at the place, see $A C$, see fridge, see the place well clean, and see the place with tiles. They are just doing these things to please us [PLHIV]... You know, that thing discouraged me completely. ...I didn't even know what to say. I just said for me, I like going to very good places. I like making myself comfortable where ever I am. Like if I come here, if I want to ease myself, I like being comfortable. ...I always like to enter toilet and if I enter there, I want to use. If you enter my bathroom, you can even eat there."
\end{abstract}

Analysis of the FGD revealed that end users require health facilities to always have adequate clean water, constant electricity, and adequate ventilation; and be aesthetically attractive.

\title{
Sub-theme 4.2.4: Health facilities has standardised processes that ensures end users spend the least amount of time seeking health care in the facility
}

This refers to timeliness of health care services. End users perceive a health care service where they are given prompt attention, and they spend the least amount of time to access health care services. Timeliness is a challenging end user requirement to attain in public health facilities, where long waiting times have negatively affected satisfaction ratings (Chow et al 2009:438, Rad et al 2009:377, Chavez de Paz et al 2009:22). Several studies have reported end user dissatisfactions with timeliness of services (Oliveira et al 2006:731; Aldana et al 2001:512; Muller \& Greco 2010:925; Gouvela et al 2011:1849; Benazzi et al 2010:861; Udoh et al 2011:222; Gutierreze et al 2009:61; Muza \& Muza 2008:12; Lima et al 2007:12; Gaioso \& Mishima 2007:1).

It emanated from the FGD that participants expect health facilities to have standardised processes that ensure end users spend the least amount of time seeking health care in the facility. Direct comments from the FGD participants are shown below as evidence hereof. 
Target process improvement measures in the pharmacy and medical records departments to remove long quoues and reduce end user waiting time:

“...if there can be any other place to somebody just go and pick your drug and go back to your house. And the pharmacist even if they can increase the number of people working or dispensing drug there, so that you don't waste a lot of time. Sometimes you go there and waste a lot of time. You finish with doctors, finish with nurses, go there and waste a lot of time. We want something if you can increase the number of people there or the branches where we can get our drug instead of sitting, sitting, sitting just like that."

"beginning from PMM, I expect that if you are coming to hospital you should spend 3hours max. Beginning from PMM, I said that if you are coming from there at least 3hours max."

End users contribute in causing long waiting time; therefore, they should be involved in developing initiatives to reduce the long waiting time:

"...I don't think it is achievable, because you see the client, apart from the manpower,
the client when they get to the doctor, some of them even headache, they'll still be
complaining to the doctor. A client can be with the doctor for about 40minutes and you
know, it'll be very embarrassing to say what are, what are... Automatically, 3hours is not
achievable. We should not deceive ourselves. But if just to come to the clinic and
access your drug, like refilling, they don't waste time. Just go to the PMM and collect
your folder and walk straight down to the pharmacy. Within 30minutes you are then off
from the hospital."

It emerged from the FGD that end users want to spend the least amount of time when ever they come to the health facility. Therefore, health facilities should involve end users in decision making regarding improvements in processes and procedures.

\section{Sub-theme 4.2.5: Government, communities and individuals have ensured a stigma free environment for PLHIV}

Stigma is a challenging phenomenon that spreads fast, except a combination of stakeholders from both government and communities work together with individuals to address its existence in the community. Fear about disclosure of HIV status and the associated stigma and discrimination is a recurring issue in most studies (Ginwalla et al 2002:707; Myburgh et al 2005:473; Coulter 2005:1200; Liu \& Wang 2007:266). 
Analysis of FGD showed that participants expect health facilities, communities and individuals to have ensured a stigma free environment for PLHIV. Below are direct comments from the FGD participants as evidence hereof.

Government's support for stigma reduction campaigns could reduce stigma:

“...many people discriminate somebody PLHIV. It will help a lot [if anti-stigma campaigns are intensified] because even your relation, if he knows you have HIV, he'll not even like to sit near you any longer. These are very important areas we need government assistance to continue to enlighten people, to let people know that someone living with HIV is not the end of his life. People still believe that may be if you are contacted and have HIV, you don't have life to live again. Therefore, things like this need constant enlightenment to let people know that contact with HIV is not the end of life."

Despite programmes and activities aimed at stigma reduction, some people (including employers and landlords) remain adamant:

“...some people still, when they hear it here, it goes out the other side...so, continue enlightenment."

"...some people still never understand or know they have it. In their working place, [there'll] just give you letter, or the house they are living, if the landlord heard they are positive, they [will] eject you from the house. So many things like that...so we need more enlightenment."

“...even though we are struggling, trying hard to bring down stigma, but you can't remove it completely in man. So, you can't...you see a situation where somebody goes to the doctor, after seeing the doctor you carry your folder yourself and go to the general pharmacy which every other person goes to. And people have come to understand that once they see this folder, this is a HIV patient."

Health care workers contribute to exposing end users to stigma:

"...there is a lady we are living with. She is my neighbour. Where she uses to get her services... when they come for ANC, they were told that if they see somebody with rashes, or vomiting too much or is lean, be careful, he is having HIVIAIDS. It is very unfortunate if at all a nurse or a health worker will say this..."

“...I have received series of complaints from my support group members and I reported to the Hospital Secretary. There are some health care workers who say this or that person is also HIV positive. This to me is not appropriate, as there is no confidentiality in care."

"...there is a particular client who said for her to receive treatment in this hospital, it is better for her to die...I had to find transport money to take her elsewhere for treatment. She has lost confidence in the hospital, due to their inability to maintain confidentiality." 
Health care infrastructure is not well designed to promote confidentiality, and stigma reduction:

"...there is no confidentiality where we take our folders. The place is so exposed that other people passing by see us, and even listen to our communication."

“...some people specifically come into the ART consulting clinics to listen to communication between patients and health care workers, in order to identify those with HIV positive status."

End users face stigma and discrimination even where they hold support group meetings:

“...we also experience challenges with lack of office accommodation. Many PLHIV who desire to attend support group meetings fail to do so because we often meet in the open, under trees... and we are usually up to 50-200 in number."

"...some people come to where we hold our support group meetings just to look at people's faces to identify who has HIV."

It emanated from the FGD that health care workers play a role in promoting stigma and discrimination against PLHIV. Therefore, to address stigma will require the efforts of every one: government, communities and idividuals.

\section{Sub-theme 4.2.6: Lifesaving ART programme is fundamental to the survival of PLHIV, thus, will not experience interruptions}

This relates to ensuring that comprehensive ART programmes are not interrupted, as PLHIV depends on the ARV drugs in order to survive. Miller et al (2002:598) reports in their findings from California that end users were willing to compromise items that may impact quality of life (i.e., increased side-effects, inconvenience, and pill burden) to have a potent antiretroviral regimen. Similar findings were reported by (Miller et al 2002:598, Schumaker \& Bond 2008:2130, Ananworanich et al 2008:329).

Participants explained during the FGD that they view the on-going free lifesaving ART programme as fundamental to the survival of PLHIV and do not expect to see it experience any interruptions. Some direct comments from the FGD participants are provided below as evidence hereof. 
End users expressed gratitude to God and health care workers for granting them access to the ART programme:

“...I am so thankful for what you have been doing for us. I don't want you to stop the drugs, because for us the money is not easy."

"...I am so grateful and happy with the drugs we receive from this facility. I am so happy for all the assistance."

"...in fact, we thank God for all the services. May God bless those who brought the doctors, those who brought the suggestions to bring the drugs, and those who took decisions to implement the programme in the name of God. We are grateful."

It emerged from the FGD that end users are thanking God and their health care workers for making life saving ARVs available to them. This could be an indication that they are happy for the opportunity to be on the ART programme.

Sub-theme 4.2.7: Health facilities ensure that any end user who turns up to the health facility benefits from full assessment of his/her current health status; and is counselled on emerging clinical manifestations that need medical attention

This refers to the need to ensure that complete personal history and physical examination is conducted on any end user who turns up to the health facility in search of health services. Results of the assessment should be discussed with the end user. The end user should also be counselled on any emerging signs and symptoms that he/she needs to know about. Coulter (2005:1199) elucidates that end users want an explanation of their symptoms, treatment, or investigation. Similar positions were maintained by AHRQ (2010:153), Coulter (2010:4), Coulter (2005:1201) and Rad et al (2009:377).

It emanated from the FGD that participants expect health facilities to ensure that any end user who turns up to the health facility benefit from full assessment of his/her current health status; and be counselled on emerging clinical manifestations that need medical attention. Direct comments that emerged from the FGD participants are shown below, as evidence hereof. 
End users insist on having their current health status examined by health care workers:

“...I want to check my weight, they said you are already too fat, don't break the scale, all this kind of something. Nobody is interested in checking my BP, except when I insisted, 'I want to check my BP!' 'I want to check my BP!!' ... doctor will now be asking me what is wrong with you ... all those kind of things. I am expecting such kind of [checking my health status as reason] for... coming for check-up ...but most times when we come for check-up, it's just to pick up our drugs."

"...like when I leave my house and come to the hospital for check-up. I know that I am coming to collect drugs, but because it is check-up, I expected that all formal medical questions should be asked, and tested. Like when I go, most times, when it is tone to go into the doctor's room, I'll say I want to check my BP. He'll say must you check your $B P$ ? I'll say I want to check. I want to know what my BP is and I want to know my weight. Even though I am fat, I want to know my weight. If there's a way I can reduce it, if it'll have influence on me."

End users perceive that when a nurse fails to examine their current health status properly, they will proceed to requesting a doctor to do so for them:

"...like when I got to the hospital and expect that I should be checked properly, since the nurse will not do the checking, I'll go inside to ask the doctor to do it; and this thing is supposed to be done and put in the records."

End users are concerned that if their health status is not consistently and adequately examined, their health condition may deteriorate into a situation that could have been avoided:

“...some of us are over aged and engaged in thoughts about this HIV infection. Yet, the hospital will not check our blood pressure. We are not able to know whether our blood pressure is rising or dropping, until something happens."

Analysis of the FGD revealed that end users are increasingly aware and insistent upon examining their health status any time they turn up to the health facility.

\section{Sub-theme 4.2.8: Health facilities recognise the essence of PLHIV as volunteers, and design capacity building and motivational programmes to meet both the task requirement and volunteers' expectations}

Support group members who provide volunteer services to health facilities could be motivated through trainings and other incentives to keep them happy to continue doing their volunteer work. In a study conducted to explore the perception of motivation and 
rewards for volunteers in health care, Akintola (2010:10) espoused that volunteer caregivers derived both intrinsic and extrinsic rewards related to their volunteer work.

It emerged from the FGD that participants expect health facilities to recognise the essence of PLHIV as volunteers, and design capacity building and motivational programmes to meet both the task requirements and volunteers' expectations. The following direct comments from the FGD participants showed evidence hereof.

Volunteers could be motivated through capacity building trainings that will empower them to make some income for themselves:

"...presently, the volunteer is still a separate issue from trying to give kind of training that will empower the support group members to adequately take care of himself. Because the volunteer just volunteers himself to going to give services to other members of the support group, knowing that he has his own personal problems that needed to be attended to. So, these two problems should be tackled differently. There's need that there is capacity building training, IGA [income generating activities] and then it should be considered that those volunteers are adequately taken care of by giving them something."

"... and there is something you said earlier about training support group members on how to do something, is also important. Some of them don't have something doing. They can be trained on how they can do something to help."

Volunteers could be enlisted as potential employees, pending any opening for employment:

"...the government should be able to employ even two or three PLHIV ...the quality of service will be better than what is obtainable now."

“...government and projects should employ or deploy health care workers and volunteers, who are themselves, PLHIV to care for us. They will understand our needs better than those who are not living with the virus."

“...I want to add on what my colleague has said. When this challenge of not employing members [of the support group] was discussed, I didn't know that [the project] will not employ. What is it that is wrong with our approach? Eight of our members are volunteers...I have each person's address... at least they need to transport themselves back home, because I thing you people go three times a week, right? ...even something for them to buy pure water..." 
Support group needs facilitation and logistics support to reach out to PLHIV through follow up and contact tracking:

"...the only thing we need is that our people in the villages... are dying from staff shortage. So we need encouragement and support groups that can help access to visit and their people in the villages as home based care services. The support group needs assistance so that they can help their colleagues that are dying in the villages. ...support groups are up to the task to go anywhere and get to that particular client, but they are not having that support. So, we need more support for support group, so that they can go and get the people from the villages to come and access their drugs in the city."

"...we expect the project to buy motor bikes for support groups to use in contact tracking and conveying ill members to hospital. This in our opinion will reduce loss to follow up."

“...it is appropriate to provide some form of support to volunteers."

Programmes working on HIVIAIDS projects could book appointments for, and introduce support groups of PLHIV to key stakeholders:

“...projects should assist support groups to open doors with gatekeepers and assist in advocacy efforts to improve their visibility and welfare of PLHIV."

It emanated from the study that end users demand recognition from health facilities. They want health facilities to design capacity building and motivational programmes to support their performance. Logistics support and economic empowerment was mentioned as a key area needing support.

\section{Sub-theme 4.2.9: Health facilities recognise that PLHIV loves interacting with their peers, and creates opportunities for such interaction as part of the continuum of care process}

PLHIV are bound by a force of cohesion, with their HIV status being the common factor which sets them up in groups. The health facilities recognise the support groups, and develop strategies aimed at ensuring continuum of care through the support group structures. According to Wagner et al (2007:871), lessons learned from Uganda, Zambia and South Africa show that to minimise end users dropping out of care, structures (such as support group for PLHIV) should be developed to enable end users provide support to each other, and have a "safety net" for helping each other remain in care. 
Participants explained during the FGD that they expect health facilities to recognise that PLHIV loves interacting with their peers, and create opportunities for such interaction as part of the continuum of care process. Direct comments from the FGD participants are shown as evidence hereof.

PLHIV have preference for association and experience sharing with their peers who are also living with HIV:

"...somebody who is positive will be more at home when handled by a HIV positive person. Somebody wey [that] meet somebody who is HIV positive and say, even me, I am positive myself. Start building... that same person will say are you positive? He'll say are you truly positive? And say, yes! Because I know, I am an adherence counsellor, so I know people who come in. I know how they feel when I tell them I am also positive. They come down well."

"...this [support group] volunteers, without you allowing them to work in freely in the hospital, because our clients understand them more than the health workers. What they can confide to us they cannot confide into even the doctors."

Support group structures could be useful platforms for ensuring quality continuum of AIDS care and drug adherence:

“...when I give health talk finish, they'll go and see doctor. When they see doctor finish, they'll come and queue for me for me for some secret things they don't want to tell doctor. So, that is the truth. If they know you are positive, they'll open up for you. There's nothing they can hide."

“...because in counselling we have the rapport more than doctors, any issue you want...we counsel ourselves. Even adherence counselling and all-round the unit, they confide in us for confidentiality. They can call me aside; I always sit in the consulting room in the department."

Support group members could be better treatment supporters, care givers and volunteers in AIDS care than people who are not living with HIV:

"...it is advisable to encourage PLHIV to serve as volunteers. Support group volunteers have the ability to cope with patients who other people will describe as nauseating and disregard. Like myself, I am a support group member working as a volunteer. I give attention to any patient coming in with whatever problem because I know how he/she feels. This is something that only a fellow PLHIV will do with passion."

"...I go to provide voluntary services in the medical records unit of the hospital. At times, I end up quarrelling with the hospital staff or being sent away; because they won't allow me help the PLHIV. There is this particular staff that does not care whether a patient 
survives. I live with HIV, so I know how it feels. Those not living with the virus have no idea how it feels, so they do not care about the welfare of PLHIV."

Support group of PLHIV could be a better platform for end user tracking and follow up:

“...activities such as patient tracking should be our job, as support group members. If you provide resources for patient tracking to health care workers, they'll report to have conducted the activity, when in the real sense, no tracking was done. That is why we experience a lot of challenges with patient loss to follow up."

Support groups are emerging meeting points for development of long term/marital relationships among PLHIV:

“...through the support group, some people have identified their love and joined in marriage. The state government has been supportive in paying bride price to facilitate marriage for those in need."

"...there is need to provide substantial assistance to support groups. Once supported, several PLHIV will be attracted to join the support group."

It emerged from the FGD that PLHIV have preference for association with their peers. The support group is a formidable platform for continuum of AIDS care, and an emerging meeting point for development of long term relationships, such as marriage.

\subsubsection{Theme 3: ART services that disappointed participants from meeting their requirements (dissatisfaction)}

This theme relates to ART services that contributed in the dissatisfaction of end users attending public health facilities. Gaioso and Mishima (2007:1) noted that dissatisfaction in their own study was predominated in terms of environment and organisational accessibility, impairing care longitudinality and continuity. Similar findings were reported by Medeiros et al (2010:402), Regmi and Madison (2010:44), Wouters et al (2008:11), Coulter (2005:1200), Coulter (2010:4) and Nakhaee and Mirahmadizadeh (2005:192).

ART services that disappointed participants from meeting their requirement was one of the major themes that emerged during data analysis. Eight sub-themes appeared to relate to this theme. Figure 4.5 shows hierarchical relationships between theme 3 and its 8 sub-themes. Table 4.5 illustrates the sub-themes that emanated from this theme. 


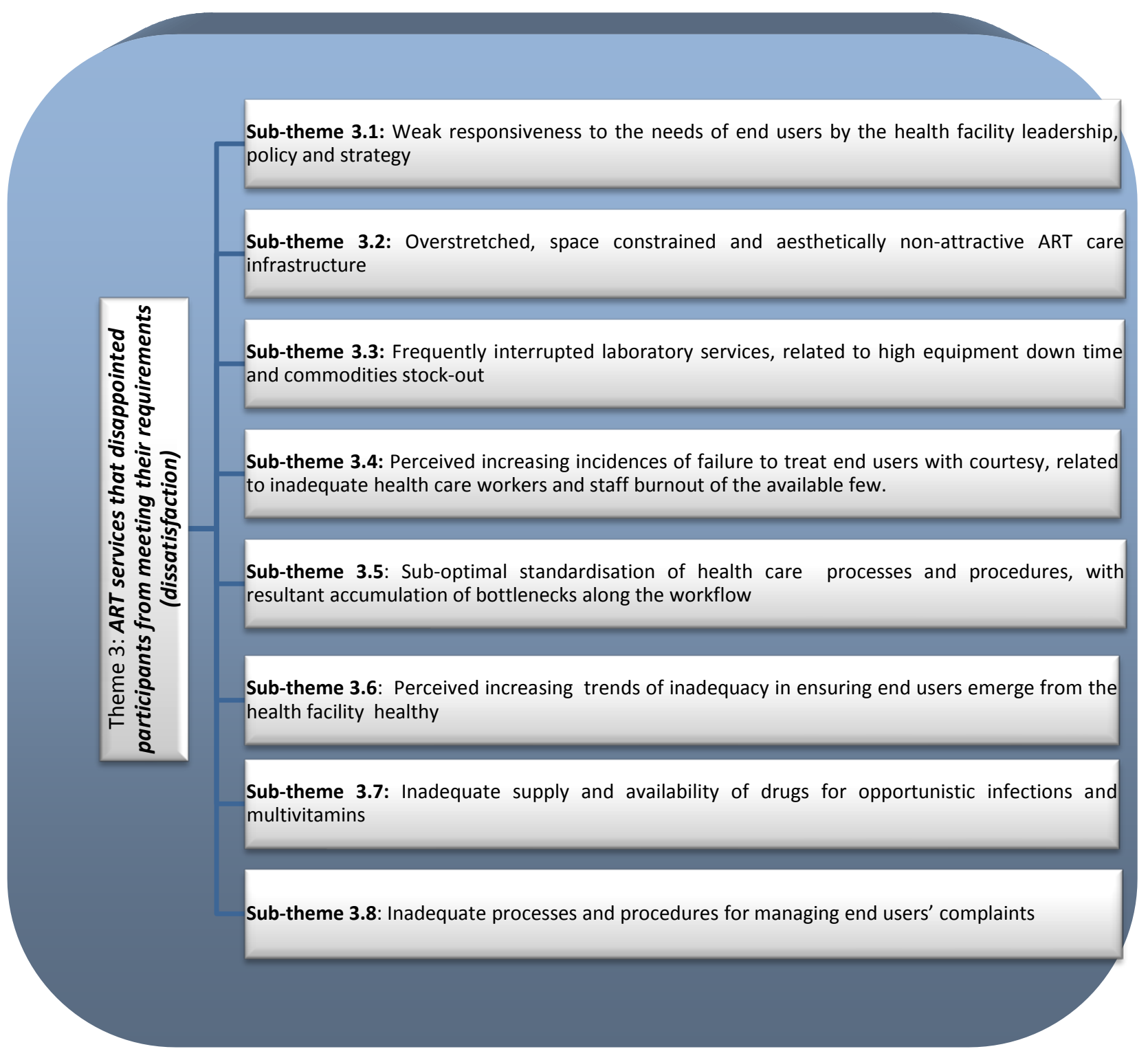

Figure 4.5 Hierarchical relationships between theme 3 and its 8 sub-themes

The concluding statements on ART services that disappointed participants from meeting their requirements (dissatisfaction) are:

- weak responsiveness to the needs of end users by the health facility leadership, policy and strategy

- $\quad$ overstretched, space constrained and aesthetically non-attractive ART care infrastructure

- $\quad$ frequently interrupted laboratory services, related to high equipment down time and commodities stock-out 
- $\quad$ perceived increasing incidences of failure to treat end users with courtesy, related to inadequate health personnels and staff burnout of the available few

- $\quad$ sub-optimal standardisation of health care processes and procedures, with resultant accumulation of bottlenecks along the workflow

- $\quad$ perceived increasing trends of inadequacy in ensuring end users emerge from the health facility healthy

- $\quad$ inadequate supply and availability of drugs for opportunistic infections and multivitamins

- $\quad$ inadequate processes and procedures for managing end users' complaints.

Users' suggestions for actions to improve the quality of ART services were to resolve:

- Weak responsiveness to the needs of end users by the health facility leadership, policy and strategy.

- Overstretched, space constrained and aesthetically non-attractive ART care infrastructure.

- $\quad$ Frequently interrupted laboratory services, related to high equipment down time and commodities stock-out.

- Perceived increasing incidences of failure to treat end users with courtesy, related to inadequate health personnels and staff burnout of the available few.

- Sub-optimal standardisation of health care processes and procedures, with resultant accumulation of bottlenecks along the workflow.

- $\quad$ Perceived increasing trends of inadequacy in ensuring end users emerge from the health facility healthy.

- Inadequate supply and availability of drugs for opportunistic infections and multivitamins.

- Inadequate processes and procedures for managing end users' complaints

\section{Sub-theme 4.3.1: Weak responsiveness to the needs of end users by the health facility leadership, policy and strategy}

This refers to the manner in which health facility leadership responds to any queries, requests or complaints made by end users. In Zambia, Schumaker and Bond (2008:2130) report that overstretched health workers may not explain a drug's action in 
the body, leaving end users to rely on past experience of pharmaceuticals and their own traditional and biomedical knowledge to interprete side effects and efficacy after they begin treatment. Failure to engage with the end user's agenda can lead to misunderstandings, dissatisfaction, and poor health outcomes (Coulter 2005:1200).

Analysis of FGD showed that participants were of the view that the health facility leadership, policy and strategies were weak in responsiveness to the needs of end users. Direct comments from the FGD participants are presented below, as evidence hereof.

Public health facilities may have the desire to support end users, but have insufficient authorities to do so beyond their limits:

“...we wrote a letter and they said the MD doesn't have the right to employ, that it is the Permanent Secretary who has that power to employ, in terms of the doctors now. They said they are going to raise a memo, I don't know if the memo has been raised or not."

“...many of the occasions I met with the management with few of my people. And what they actually discussed with us and they go around finding out if the report was true. That was how I knew that some of the doctors died, some left for other jobs while they now have 2 or 3 left in the facility. If not that, I wouldn't have known."

“...she was not having money. They told me to borrow her money, I said I don't have. We followed her down there, they admitted her pending of when the husband will come and pay. So, they have open policy to clients. They don't restrict anybody whether you are PLHIV or not. I believe the policy is to the betterment of the patients not that of the management."

Participants perceive that changes in health facility leadership might have contributed to weak responsiveness to their demands:

“...based on my own observation ...the administration is not giving us enough support...the support we are expecting is to give us space where we can meet as support group. If we have a problem with the hospital, we should take the case to the administration and they should give us forum to tackle the case."

"...most of the challenges we started having is that they transferred all the doctors and [MD] who recognised the support group. So, immediately these people gone, we started having problems. Even our current [MD] now, he doesn't recognise support group..."

“...this new [MD] has no concern about us ... [the former MD], on support group day we see [him] himself. He'll come to the support group. But this one, I don't think he knows the venue of our support group." 
"...in our own case, they [health facility leadership] discourage [support group] volunteers, even me; they tell me and my sister that they don't want to be seeing us in the clinic, which is very bad."

“...we reported cases of lack of courtesy treatment by health care workers but no action was taken. The most aggressive of all staff, we understand is the medical director's brother, so he feels he is above the law. He does whatever he feels like doing to patients, and you can do nothing about it."

"...there was a recent case of insults he rained on one of our officials, but nothing could be done. Some people said he is not a hospital staff, but project staff."

Some health care workers demonstrate interest to be responsive to end user demands, but lack necessary authority to go beyond their authorised limits:

“...that ART coordinator...is supporting us [support group], but he has not power over the [MD]. Anything I tell him, he'll like to approve but he doesn't have power to approve anything. Like the time we had problem with the [MD], that time we [support group] were doing [conducting] health talk every morning. There is no nurse to talk to them. I come and do it myself."

Leadership support for support group activities is encouraging in a few health facilities studied:

“...in my own facility, we thank God that they are $100 \%$ in our support. Because without supporting us, that is, what we are getting in that place concerning to this quality of a thing, we wouldn't have gotten it. In the Heart-to-Heart, even the [MD] knows of our existence. Because there's nothing we'll do without them giving us go ahead. Because the hospital belong to them, they are the ones that administrating the hospital. Because we are under them and we take permission for anything we want to [do]. And since the support group was inaugurated, there's nothing we ask from them that they refuse to give us. Even when a time came that we wanted an office, they gave us."

“...like every morning, they'll give health talk. The [MD] will come out and make sure that anywhere, everybody is comfortable. Anything you need, any questions, anything that is disturbing you, you say out your feelings..."

“... at times, we don't even get to him [MD] before our problem is solved. What is the point of getting to him as long as we know at the meeting if the challenge doesn't confront me...sometimes, even if not me seeing the [MD] during the visit [with] the different departments...I get what I need from [them]."

"...in the past, our clients come out from the consulting room and stand outside the pharmacy. Then, myself I went to [the leadership] and explained that look, look the way our people are standing. I need to assist them. Then ...they put shade there. Actually, it's very, very encouraging."

"...hospital leadership is good and cooperative ..." 
"...the hospital leadership is very cooperative and responsive to the pleas of PLHIV. We recently reported a case of a health care worker who disclosed a patient's confidential information to others. The hospital took prompt action and ensured the staff was demoted from GL14 to GL12."

It emerged from the FGD that inadequate authority level or changes in health facility leadership could cause weak support for support group activities, and thus, reduce end user satisfaction.

\section{Sub-theme 4.3.2: Overstretched, space constrained and aesthetically non- attractive ART care infrastructure}

This finding relates to the physical or infrastructural and environmental outlook of the health facility. It includes availability of space for comfort of end users and physical appearance of the infrastructure, including aesthetics. Pietrovsk and Dall'Agnol (2006:630) indicate aesthetics and cleanliness of the health facility environment as factors that interfere with end user satisfaction. Similar findings were reported by Gaioso and Mishima (2007:1), Medeiros et al (2010:402), and Agha and Do (2009:87).

Participants explained during the FGD that their ART service sites are overstretched; experiencing major space constrains and aesthetically non-attractive to sight. Direct comments from the FGD participants are shown below as evidence hereof.

End users attending ART services in the health facilities are overcrowded beyond the space originally provided for ART services:

"...most of the time, the patients are many. At times the seat is not enough. At times, they'll hang around, because the more we register them, the more the place is becoming too small. So there are times when you come here and actually there's very limited space to sit, even with all these chairs."

“...most of them [other ART sites in the State] are too congested. Even here is better, for me is even lesser than [others]. ... [You go to the health facility] in the morning before you leave that place is already $4 \mathrm{pm}$. You'll be waiting, waiting, waiting, continue waiting, in fact people are lesser here."

“...the place [health facility] is too crowded and the room is just $4 X 10$, which 2 or 3 doctors sit and see PLHIV and also receive other patients [in the same space]. The place is too small for them. When you get there, the place is too hot ...the place is too hot for the nurses, for the doctors and for the patients together ... before you know it, one of them will be annoyed with one another whether because of the heat and all that." 
Constrained storage space and inadequate lighting in the health facilities:

"...where we do our filing is too tight. We have no light. Whenever NEPA [National Electric Power Authority] takes light, we have no light to sort [files]."

“...we don't have space [in the health facility]. This morning the way I kept things, because if I have patient I'll bring out food flask, drinking water, water flask, plate, all these things. Where I have to keep those things, where I brought them out this morning is in the toilet.

When I have patient, yesterday they brought a patient to me in the evening so this morning I cooked so I want to fetch the food inside flask. I have to go and bring my plate where I kept them inside toilet ...there's a little way, a little store there. You pass through the toilet before you see the store. There's a space there; that's only where I can put those things so they can be safe."

“...those clothes, the 'Ghana must go' [a big sack]. I told you that somebody brought for us, I took it to antenatal hall, where they opened for me to keep those things inside toilet. It's inside toilet they opened for me to keep those 'Ghana must go.' Those are the things we are passing through... this morning, I have to bring out plates from toilet to put patients food inside, because no place to put my things."

Inadequate number of seats to match end user population on clinic days:

“...go and witness it yourself. You'll see our patients standing, there's no enough seats. Really, the care is not there; there is no care, there's no care..."

“...we have limited number of benches in the clinic waiting areas. Sometimes you find several patients sitting on the floor, because they have no seats to sit on. Yet, we are told it is not appropriate for patients to sit on the hospital floor. This needs to be addressed."

“...no, we don't have enough chairs. The only place that we are taking our drugs now, the environment is not conducive enough... I normally go there every Wednesday, because is our clinic day. Then, the environment there, outside where there is chair, there is no body to sweep. They don't sweep the place. They don't clean the chairs."

“...When you come you'll see our clients lying on the floor, because there's no seat. Those that are weak, they are lying on the floor. Just somebody go, just go and take your eye and witness what we are saying. You'll see patients lying on the floor."

“... in the PMM, they don't have enough chairs because I remember about 2 weeks ago, the support group have to send about 20 chairs which we bought for them, for the patients to be sitting on. The other chairs they have been not enough. But for pharmacy, they have enough chairs, because they have long, long benches which can contain a lot of people. Then for social welfare, they also have chairs because people who go in there are not much like in PMM and pharmacy."

"...the same thing in PMTCT, there is no chair there. Pregnant women that attend at times are more than 80 . So, you'll see some pregnant women, they'll be sitting on the 
ground, some standing, no chair. And before you talk to 50, all those space, no fan, no water, the little office there, no ventilation and 3 people working inside that little office."

ART clinic environment is unattractive and non-condusive for end users:

"...the environment where doctors see patients is not conducive."

"...we have problem with ventilation. Look at the way they constructed the place. They didn't do it in such a way that there'll be ventilation."

"... and when you get there, there'll be dust on the chairs, everywhere, if you don't have toilet roll you'll be looking round to see how to dust the chair before you seat down."

"...the [health facility] environment is not conducive like we see [in other health facilities], so for my own observation ...there is need to know forward where they can assist the clients who are picking their medicines here..."

"...the [health facility] environment is not really attractive. There should be a cooling whatever, like an AC [air conditioner] ...the place is always congested, crowded, so we need that $A C$ to make the place accommodating ..."

Participants from 1 of the 6 health facilities investigated said their health facility is clean and attractive:

"...in terms of may be making the place too beautiful, may also be a problem because there's a client that came to me. ...I was counselling this client; he was like...if this thing is a good thing, why are they making the place look so special? Just look at the place, see $A C$, see fridge, see the place well clean, and see the place with tiles. They are just doing these things to please us [PLHIV]... You know, that thing discouraged me completely. ...I didn't even know what to say. I just said for me, I like going to very good places. I like making myself comfortable where ever I am. Like if I come here, if I want to ease myself, I like being comfortable. ...I always like to enter toilet and if I enter there, I want to use. If you enter my bathroom, you can even eat there."

...for cleanliness of the environment, we are Ok. From lab to MOPD, to Heart-to-Heart to ANC, are all very clean."

"...hospital environment is always clean, as the compound staff sweeps every day."

Analysis of the FGD revealed that health facilities providing ART services are overcrowded beyond their space limits. They also experience constrained storage space, inadequate lighting and unattractive environment. 


\section{Sub-theme 4.3.3: Frequently interrupted laboratory services, related to high equipment down time and commodities stock-out}

This finding relates to the frequency of breakdown of laboratory equipment and laboratory commodities stock-out, resulting to interruptions in laboratory services provision. Alemayehu et al (2009:360) report that a relatively better quality of laboratory services was provided to end users on enrolment into care, but failed to sustain the care during follow up care. Mfinanga et al (2008:3) report a similar dissatisfaction with laboratory services.

It emerged from the FGD that participants felt they are constantly frustrated with the frequently interrupted laboratory services, arising from frequent equipment breakdown and laboratory reagent stock-out. The following direct comments from the FGD participants are evidence hereof.

Equipment breakdown and laboratory reagent stockouts formed common causes of interruptions in laboratory services:

"...there are sometimes we come here to do CD4 count. So many times, we come, no facilities to do the test. Still, they don't allow somebody to do the test outside. Sometimes, some people like I, came here more than 5 times ...for some of us, we don't always have time. When you come, I don't know the name, OK, reagent, there's no reagent. You have to continue coming. Sometimes when you want to collect your drugs from the pharmacy, they'll tell you without the CD4 they'll not give you drugs. So sometimes, we don't know what is happening like that..."

"...for lab, the equipment there is Ok. But at times, the machine do breakdown. That is the CD4 count [machine]. The other time, they were complaining that there was no reagent for the testing."

“....at times, the reagents will not be there. At times, they'll be there. For about 3 weeks ago, there was no reagent. When they now supplied the reagent, the CD4 machine was bad."

“...the lab, apart from shortage of CD4 machine which sometimes breakdown and reagents, they are equally doing well. When they have materials, they attend to everybody, no matter the time you come. Their attitude is good."

Number of end users on waiting list for laboratory tests places excessive burden on the laboratory equipment, with eventual breakdown:

“...in the area of lab, the machine is always over used. Even the one that they brought recently. So, when they now removed that one and brought the new one now, the thing 
did not last up to one year. It started developing problems again. And because we are having problems with that CD4 machine, to monitor somebody is always a case, [especially], the newly initiated. Because it is when the person now go on CD4 count that is when you know the level of CD4 count in case you want to start ART."

"....and then the CD4 kind of a thing ...I don't know what is actually happening there. The only thing we know is that CD4 count is always having problem as far as some of them are still there. What some of them tell us is that the load is too much. I know that in spite of that thing, I don't know if the load is really too much."

Weakness in laboratory systems, including inadequate health care workers and suboptimal capacity to plan effectively, interrupts laboratory services provision:

“... recently, the hospital could not even detect TB; you had to be referred... I don't know now, but needs to be improved."

"....in the part of CD4 count now, like somebody like me now, it's almost a year now I have not done my CD4 count. Any time I come to the hospital, they'll say there is no reagent."

“...concerning our clients, we are a client for the CD4 count that is not for the general lab. They [lab staff] are trying their best because we can just say they have lesser things, manpower, because at times, if our people are so many, there's no how there'll be specific on number that they'll take. Others will have to go back. So, you see all those processes, you don't really know because some of this our clients come from a far of places."

“...you can just imagine only one machine for 100 or 80 for CD4 count [a day]. There is no way one person [can do all]. This must be time consuming, especially now that the reagent is even scarce...some of these new clients, there is no how the doctor can prescribe their drugs without seeing their CD4 count."

\section{End users pay the price of poor quality caused by inadequate health systems:}

"...we enjoy services in this facility. However, we have challenges with the lab. You'll travel a very long distance from home to the facility. Then, you are told that they won't attend to more than 15-20 people for CD4 tests that day. Then, you pay transport again back home without accessing the CD4 test. Yet, it is fundamental to have the test as part of the care process."

“...sometimes when you come, they'll say only 20-40 people will be tested that day. That is where we encounter challenges. The problem is not with the health care workers alone, but the system. Sometimes, they get limited supplies of working materials, not exceeding enough for 20-40 people only."

“...sometimes, the CD4 machine itself will fail to function. It takes a couple of days or weeks before the machine is repaired, and function again." 
Analysis of the FGD revealed that end users pay the price of poor quality caused by inadequate health systems: weak laboratory management systems, inadequate health care workers, excessive burden on equipment and frequent lab equipment breakdown.

\section{Sub-theme 4.3.4: Perceived increasing incidences of failure to treat end users with courtesy, related to inadequate health care workers and staff burnout of the available few}

This finding relates to instances where end users feel unfairly treated, or given treatment without passion. This often stems from inadequate availability of health care workers. Staff burnout could also cause treatment of end users without considerations. Udoh et al (2011:221) hypothesise that the mode of health care delivery appears to be more important to end users than the actual care itself. Similar findings were reported by Chow et al (2009:438), Coulter (2005:1199), Yakong et al (2010:2431) and Gouveia et al (2005:S109)

Analysis of FGD indicated that participants were of the view that the health facilities increasingly failed to treat end users with courtesy; most likely caused by the inadequate health care workers or burnout of the few staff currently available. Direct comments from the FGD participants are illustrated below, to buttress this finding:

End users face experiences of discrimination, intimidation and being scolded by health care workers:

“...the nurses they call matron or something like that, the way they shout on people having this virus. It is as if there's no, or you are no more a living person. They will see you now [and say]..."please, see me, see me, na me give you sickness?" They are not even telling us that there will still be life. That is why I always ask ... may be they don't know how someone in this problem feels... In fact, they are very harsh. ... may be the person's mouth may be having odor or your ear sef may be having problem, not able to hearing something clear. Something like that. They'll be shouting at you, that's my own experience, these are not even exaggeration or saying they say. That is how they did it to me and up till now, that's how they are treating patients, especially, new patients there. My own is easier...the way they handle them [new patients] is very, very bad, very poor for their services."

“...there is a particular staff member, responsible for giving out folders. He is very harsh towards PLHIV. He has a very bad attitude... most of our members have stopped taking medications because of him. If he sees you, he won't smile... like fight, he's just too aggressive." 
"... another area that we have a very big challenge, I wonder why people did not raise it up is in the area of admitting members. They don't normally admit members [PLHIV]. The doctor will tell you that nobody should attend to that client, either in the night or at day, that he wouldn't have time to do ward round ...they will just write drug, prescribe drug for that patient, then let the patient to go back home."

"...I have no challenges with the doctors and medical records staff, but I go to the pharmacy with my prescriptions of ARVs and Ols even when I'm very sick, they'll not attend to me speedily. They tell me to leave them alone; that they are very busy."

"...when I ultimately see a doctor and go to the pharmacy to pick up my drugs, the pharmacists won't even look at me. They'll tell me they are too busy to attend to me. This is frustrating."

"...we need more efficiency in the pharmacy, because sometimes we are being stigmatised. The behaviour is compromising. We are very Ok with nurses and doctors, but we are discouraged of coming with the attitude of pharmacists."

"...like there was a time I went there [to the pharmacy] and said please, can I have your biro. The man incharge now 'yab' me, as if I am not a human being. That see, just ordinary biro, get out don't take chance. [He] just drove me out of that place, that I should get away from this place and go and get biro...it is devastating..."

\section{Dearth of skilled and experienced health care workers to provide quality ART services:}

"...in that regard, I want to say that it is not always that it is like this. It use to be, but with the Corpers that were working before this strike, people are being admitted ...it is still the same thing we are talking - manpower is still a problem."

“...then [hitherto], we had up to 7 or more doctors attending to PLHIV. But now, is only 2 doctors that are around now taking care. As I'm talking now, we have only 2-3 doctors upstairs."

“...the ones [staff] present now cannot carry the load because people are more. People are coming in more and more. The number as at 2008 compared to the number today, the PLHIV are no longer the same. They increased!"

“...the capacity in the general hospital is low. We are having problem because we don't have enough doctors. Like before this exercise, we have only one main doctor, then about 10 they are Corpers. Sometimes if somebody is down, for the person to be admitted is going to be a problem because they don' ...have enough doctor ...they'll be complaining of work load."

“...as I was saying that area of manpower is the problem they are having at the MOPD...if the problem of manpower can be solved, they may be very perfect, they are doing work."

"...the services I receive is good but we actually need more doctors in the [health facility]." 
Health care workers are inconsiderate in meeting the demands of end users:

“...there was a day I was given appointment to come and collect my drug from the pharmacy. They gave me appointment from the place so I came. He said go, go away, you just collected about 2 weeks ago and you are just coming now. I said because of the nature of my job, this is the appointment they gave me ...he refused, and sent me away. You know some of us we just take small opportunity for working place to come. Some of us we have no chance at all to come here. Even, I'm supposed to be on duty now. I had to sneak out, told them I want to go and buy something. So, things like this, when we have opportunity [to come to the health facility], you have to attend to us."

“...there is one particular area that everyone is concerned about. This concerns a particular person who will not attend to you if you forgot your hand card at home. Even when you tell him your number, he'll not listen. Rather, he'll start harassing you as though you are a kid. He does not do this to me alone, but to everyone attending this clinic."

“...the hospital should provide temporary accommodation facilities for patients who come from very long distances to access ART. Efforts should also be made to expand services to bring ART closer to individuals and communities."

Health care worker burnout as a result of excessive end user load:

"...it's like the work is too heavy for her [the doctor]. Sometimes I find it difficult to bring out all my complaints to her in order just to see her. Because the crowd there is too much. When you go to her, she looks tired. She looks tired. She'll be looking through the window. There was no light, she wasn't comfortable ...she was complaining that the [work] load is too much for her."

“...most time recently, they [doctors] don't have time for that [asking or giving information to us] at all. Even, before they'll ask you any complain, they've already filled your file."

“...we [PLHIV] are many here, we are many and staff they are always busy doing one thing or another. You can't come here and find them sitting idle, without doing anything. So, they have to be from one table to another, from one table to another, and they use to be very busy for long hours. We work with them so we know what is going on here."

\section{Despite popular opinion among participants on dissatisfaction with courtesy of health} care workers, very few end users confirmed that they were treated with passion:

"... we do not encounter many delays when we come to pick up our drugs from the hospital. We usually spend between 1 hour and 1 hour 30 minutes; and may be a little more, depending on patient load that day."

“... whenever I come there [ART clinic], they'll call me and some of them normally attend to me like my sisters. Especially, to the doctor. When I went there last time when I was admitted, he asked me many things. He said, how am I feeling? How is the sickness?" 
It emerged from the FGD that end users face discrimination, intimidation and scolding from health care workers. Dearth of health care workers contributes to frequent staff burnout and suboptimal ability to meeting end users' dynamic demands.

\section{Sub-theme 4.3.5: Sub-optimal standardisation of health care processes and procedures, with resultant accumulation of bottlenecks along the workflow}

This finding refers to inadequate realisation of standard processes and procedures in order to achieve end user focused ART care. These inadequacies gave rise to stasis or slow down of required process outputs. Findings reported by Wouters et al (2008:11) showed that, because human resource shortages are associated with lower levels of end user satisfaction, the quality of care is definitely affected by heavy workloads. Similar findings were reported by AHRQ (2010:147), Wouters et al (2008:11), Wong and Bradley (2009:253) and Wagner et al (2007:871).

It emanated from the FGD that participants opined that health facilities implement suboptimal standardisation of health care processes and procedures. The following direct comments from the FGD participants illustrate evidence hereof.

End users come out as early as 5 in the morning and spend long hours in the health facility, waiting to access ART service:

\footnotetext{
“...so, we line [queue] up as early as 5-6am [in order to get treatment early]."

"...the waiting hour is too long. And I think it is because the manpower is limited."

“...on clinic days, you have appointment, you leave your house as early as 5.30am, before you go back to your house will be around 4 in the evening. So, if there can be any other place to somebody just go and pick your drug and go back to your house. And the Pharmacist, even if they can increase the number of people working or dispensing drug there, so that you don't waste a lot of time. Sometimes you go there and waste a lot of time. You finish with doctors, finish with nurses, go there and waste a lot of time. We want something if you can increase the number of people there or the branches where we can get our drug instead of sitting, sitting, sitting just like that."
}

Identified process improvement gaps are left unresolved, due to inadequate capacity and shortage of skilled human resources:

"...we have challenges from the filing section [medical records], seeing doctors [OPD] and collection of drugs [pharmacy]. We have tried fixing these, with no good results. Especially, separating files for those who want to see the doctor from those who just 
want to pick up drugs. It is at this point that even those who came early will have their files mixed up in wrong places. Thus, causing unnecessary delays for the patient. This is the greatest challenge we have in this hospital. We have worked to improve on this challenge for over a year now, with no good results. When I ultimately see a doctor and go to the pharmacy to pick up drugs, the pharmacists won't even look at me."

"...you see a situation where somebody goes to the doctor, after seeing the doctor, you carry your folder yourself and go to the general pharmacy which every other person goes to. People have come to understand that once they see this folder [which looks unique], this is a HIV patient."

\section{Support group leadership serve as end user advocates and intervene at critical times to} facilitate access to treatment for their peers:

"...most of the time, even this morning, there's a patient that came. Her appointment was last week. ...now, if you go to the clinic after 9am, they won't attend to you. Those boys bringing folders out, they will tell you, you are late. You should go back. So, this patient's appointment was last week Wednesday. She came; they sent her away that she is late. This week Wednesday, she came, they couldn't find her folder. I now followed her to the M\&E [medical records unit], that what are we going to do? What advice are we going to give this patient? How are we going to help this patient? They said I should keep the card with me, after clinic they will take time and look for the folder. So, such cases they will say come tomorrow, come tomorrow, after one month...they will open separate folder...this patient she is on drugs and drugs is finished, come tomorrow, come next tomorrow is not helping matters."

"... after the health talk, the way we'll be going line by line, booking in seeing doctor. After seeing doctor, we'll now be going line by line to collect our drugs, so that there'll be no confusion in the process. They make sure everything, they arrange everything, they take proper care of us, even they encourage us, most of us to be attending support group ...they announce to people to come and join support group because it use to help."

\section{End users brainstorm and come up with innovative ideas on improving processes,} removing bottlenecks and reducing long waiting time:

\footnotetext{
"... when we discovered the situation [delays in folder retrieval], I met my people in the support group and said this is what we shall do. We went to the management, the management agreed with us. They allow us, on Tuesdays; they will sort out all the folders [preparatory to the clinic day on Wednesdays]. When they sort out these folders by 7am; 8am you will see the doctors already in office. Now, our clients, even last Wednesday, we were almost about 180, but before 1pm, they've finished accessing their drugs."
}

\section{End users bear the cost of health care workers poor quality:}

“...the CD4 count, they said the doctors didn't write the file number on the file. That one they won't put it in the files of the patients. When the patients do the CD4 count, to see the doctor now, you won't see the CD4 count result. So, the people that looking for the 
file, dey incharge of that thing. I went to them and said that how manage, about 10 people, including me that very day [have no results in the file]. I went there and said what the reason is? He said because the doctor didn't write the file number on the form. I said Ok, I started going to doctors round that they should write that number so that it should be there. So, when this thing getting too much, I tell...the person who in charge of that, that give me that file that you are putting CD4 count, the one that is not in the file, because it full file. I said let me help you call the names so that before they start seeing doctors. They refused, saying is not our job."

“...there was a client that did CD4 even twice, and they can't find the result. So, I had to go to M\&E [medical records unit] to ask. Please, let me have the CD4 folder to find for my clients' results. When I started going through, I discovered that the one in 2010 they were mixed with that of 2011. [I told him] that ...there is a lot of work here that I can even do, to help ...even to arrange [the folders]. He said no, no, no, the madam [unit head] will not agree. The madam will not agree. As I was searching for that result ...she [the unit head] came and started, madam, madam, madam, cause problem, madam, madam, blow language there, carry her bag comot [carried her bag and went out]. [The staff] said this is what he was telling me. 'We are sabotaging,' this is what the madam is saying."

“...you see, like in that PMM [medical records unit], most clients come [the previous evening and sleep in the clinic, in order to have their folders sorted out early], but that was long ago. [Then, the PMM staff] come to the clinic may be after 8am. But now, $6 \mathrm{am}$, because one of the members of the support group is among them; 6am all the volunteers are in the office. Before that, they've already sorted the folders out. The folders prepared, they'll move the folders to the antenatal hall where clients are sitting down. After the health talk, and adherence on their drugs, what to do and what not to do. When it is 7.30am, they start giving them [clients] their folders. As they give them out their cards, members of volunteers that are in the PMM will make sure that even the folders that they couldn't get, they'll get it. So, not that when you just bring your card they just go to the archive and sort the folder out, but for all the appointment, people that are supposed to come that day, their names has been written down. So, if it is 120 clients we are expecting, the folders are already there."

\section{Syndicates use health facility's inadequate processes and procedures as opportunity to estort money from end users:}

"... when they asked me to come for CD4, I use to come from [a long distance]. Sometimes, I may even leave home around 5am, to make sure that I'll come on time. Because they may say that they need only 40 people, so I'll struggle hard to come early with my daughter. My daughter is also supposed to do CD4. By the time I reach there, I may see up to 10 people there, but when I look at the form that they do give, the form may even pass 50. Then, I'll start wondering where all these people... I did not even see number of people like the form that they keep there. Then, there are some people that I hear that there is somebody that they use to pay him. If you come tomorrow, they give that person money because she lives around here. Very early, she'll just pack form to come and keep there because she has already collected money from those people [or some have promised to pay after the service]. You that came early, they'll say that your own has passed. Then, I'll have to go back, the next day I'll continue coming. I've not even done [the CD4 test] for the past one year. So, I have to go at Teaching Hospital to go and do the test. Then, when I did it there and bring the result, the doctor rejected it, and said that he'll not accept the result." 
"...from my experience, it is N500 we use to pay [to get an early slot for the clinic day]"

"...sometimes we run out of continuation sheets or forms from our folders. We suggest the provision of photocopier machines to aid in quickly making more forms when they are not available."

It emanated from the FGD that end users face extortion, long waiting time and bear the cost of poor quality in public health facilities.

\section{Sub-theme 4.3.6: Perceived increasing trends of inadequacy in ensuring end users emerge from the health facility healthy}

This finding refers to end users' perception that health care workers are increasingly failing in their responsibilities to ensuring end users get well soon and go back home in good health. To maximise impact on treatment outcome, it is important for ART programmes to develop effective mechanisms for coordinating care to ensure that end users emerge from the health facility better than the way they came (Wagner et al 2007:871). A similar finding was reported by Hopkins et al (2009:927).

Participants explained during the FGD that end users perceive increasing trends of inadequacy in ensuring that they emerge from the health facility healthy. Illustrated below are direct comments from the FGD participants as evidence hereof.

Participants perceive that quality ART service provision is increasingly deteriorating with increasing number of new enrolments:

“...I started ART about 2 years ago. The very first day, the way I was received into the family of this HIV issue gave me encouragement. The way I was received right from the Heart-to-Heart, how I was being counselled, how I was being directed to the doctors and the way I was taken for the various tests, they kind of gave me encouragement."

“...like in the PMM, we sometimes have some of our pharmacy forms in irregular."

“.... when we started, it wasn't like that...you can do your CD4 at any time... You can come, have your appointment on a Wednesday, do your CD4 count on Monday; by Wednesday it is done. And now because of all the jam packing of all these things. At times even this CD4 count is because the nurse is not there."

“...there is a particular woman whose baby encountered problems after delivery. Due to poor health care quality, she lost the baby... we were referred to someone I think was not knowledgeable in this work. ...he pressed the baby's abdomen until faeces came 
out from the anus. This was in the evening. By the following morning, the baby was dead. I view this as share poor quality health care."

“...they don't do us BP [blood pressure], because they say we are too many. Before [under the previous leadership] they do us everything. They start from vital signs, then weight, they do you BP, then, before you'll start seeing doctor. Since this new people [leadership] came ...there's nothing like that. They only do us weight. Then I requested and said, this thing is very important because somebody's BP may rise he'll not know, how will he know? They said that we are many, so they'll not be doing it."

Health facilities are not at the same level of user focused quality:

"...you know, seeing is believing, the type of compassion we are receiving in [our health facility] should extend to other areas [health facilities]. We call them our sister hospital ...because sometimes if our [CD4] machine is having problem, we send some of our clients to [the other health facilities] but when they come back, they tell us what they see there. Honestly, they'll not even like to go back there."

"...[X] hospital is very sympathetic at times, when I hear the type of suffering. This is my colleague the coordinator, we meet in coordinators meeting some times. The privilege they gave us in [our health facility], I don't know where the lapses are coming from, whether from the coordinator we can't say."

It emerged from the FGD that there was increasing deterioration in quality of care, with increasing number of new enrolments into ART.

\section{Sub-theme 4.3.7: Inadequate supply and availability of drugs for opportunistic infections and multivitamins}

This finding relates to frequent out of stock of drugs for opportunistic infections and multivitamins. Karunamoorthi et al (2009:331) sought to determine end users' level of satisfaction and their expectations with pharmacy services at specialist ART unit in the government health facilities of Addis Abbaba, Ethiopia. Results of the study showed that end users faced inadequate supplies of drugs, but could not tell what the cause of drug outages were (Karunamoorthi et al 2009:331).

It came out from the FGD that participants felt end users experience inadequate supply and availability of drugs for opportunistic infections and multivitamins. Outlined below are direct comments from the FGD participants as evidence hereof.

Health facilities experience outages of Ols and sometimes, second line ARVs:

"...in the case of Ols, we pay for Ols, unlike other sites..." 
"....another major challenge we are having is the issue of Ol [drugs]. The problem we have is OI [drugs] and that is why some of our people prefer going to where they [will find free OI drugs]"

"...we get our ARVs each time we come for it. We only encounter challenges with drugs for Ols, which are usually out of stock. Sometimes we also experience stock out of second line ARVs."

"...drugs of Ols should be made available for us in the hospital, to address our rashes and other Ols; because most patients cannot afford to buy the drugs."

Nutritional supplements are not consistently provided in the health facility:

“...the hospital should provide nutritional supplements or food, such as beans, Bournvita, and other food items that are provided for patients in other hospitals."

It emerged from the FGD that end users were made to pay out of pocket for nutritional supplements and OI drugs.

\section{Sub-theme 4.3.8: Inadequate processes and procedures for managing end users' complaints}

This finding relates to deficiencies or none availability of defined processes and procedures for entertaining or managing queries and complaints from end users. Ding et al (2008:40) elaborates that care can be poor even at sites with good support services if there is not a suitable health care worker relationship. Similar findings were reported by Madeiros et al (2010:402) and Wouters et al (2008:210).

Analysis of the FGD showed that participants were of the view that when they experience challenges in the course of seeking quality care in the health facility, they lack guidance on who to contact to lay their complaints or grievances with. Direct comments from the FGD participants are illustrated below, to buttress the finding:

End users have complaints to file against health care workers, but are unaware of the procedures for doing so, or processes for complaints management:

"....at a point, after waiting [to be tested in the lab] for so much hour, some of them will be asked to [go and] come again. It is not obtainable outside Nigeria that is being done here. Sometimes I use to hear that there are certain mistakes that the doctor will do and the patient will sue that doctor, but that cannot happen here. I have heard cases of a 
doctor throwing away patient's folder probably because he was annoyed because they know that nothing will happen. And if, just like my sister said, I wish to know if she is denied of the right to collect her drug on appointment day, what does she do? Or what [will happen to] that person who refused [the drug]."

“...my wife delivered here...she got her PMTCT here. When she went there, they registered her. She was accessing PMTCT here but they billed her for the drugs. Yes, you know you pay, but the free is still free; but they don't tell you which one is free and which one you are paying for. Then, because she didn't pay N1500, she was [not given some drugs]. I had to go there the following day myself and said I want to see the [health facility head]. My wife is HIV positive and she registered for PMTCT. She came with so, so drug. The doctor said you know the drug, we tried to pass to pharmacy or no, she didn't pay. I called the [health facility head] they couldn't allow me to see the [head]. So the doctor and the matron were begging that it was a mistake, that they were giving her aqua. ...they play on our rights..."

“...because we don't have enough doctors, some people that want to see doctor they will not see the doctor concerned. By the time the might have seen the doctor, they come the following day to take their drugs [because the pharmacists wouldn't attend to them at that time]. I don't know whether you people can even talk to the pharmacists, that in as much as some of our members are still there, let them wait for them to give them drugs, because we are not staying in that place because we want to stay there, but because doctor did not see them on time."

It emanated from the FGD that end users were unaware of health facilities' processes and procedures for managing end user complaints. Therefore, they lacked ways of dealing with their concerns.

\subsubsection{Theme 4: Participants' perceptions regarding their requirement for improving quality of ART services provision in public health facilities}

This finding relates to participants' views regarding their requirement for health facilities and health care authorities to adopt in improving the quality of ART service provision. Mainly, participants center their requirements towards improving end user focused care in health facilities providing ART services. Antiretroviral therapy service provision requires high end user involvement in the consumption process, and the traditional health care view of technical quality is inadequate to manage this complex (Gill \& White 2009:14).

Participants' perceptions regarding their requirement for improving the quality of ART service provision in public health facilities was one of the major themes that emerged during data analysis. Four sub-themes appeared to relate to this theme. Figure 4.6 shows hierarchical relationships between theme 4 and its 4 sub-themes. 


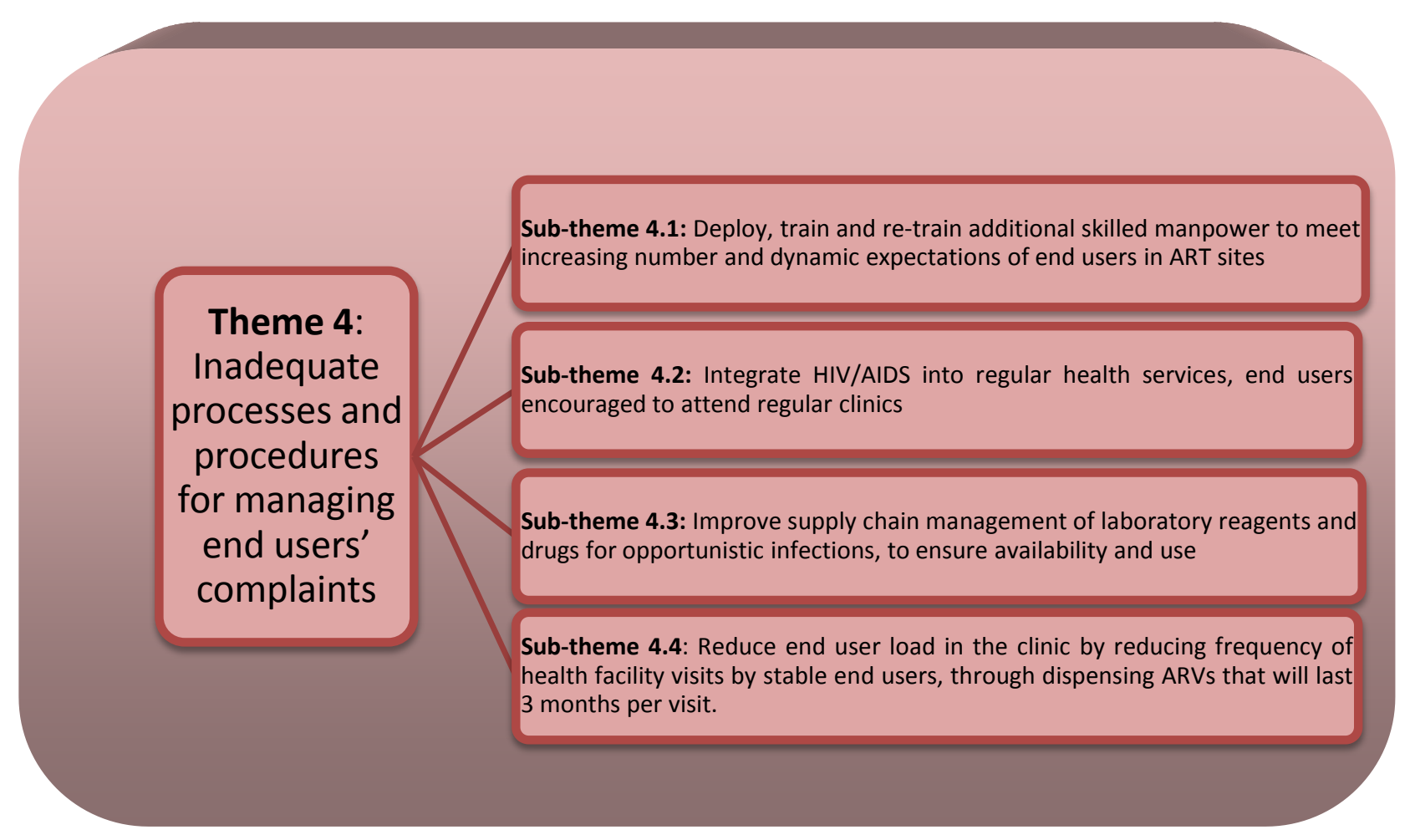

Figure 4.6 Hierarchical relationships between participants' requirement for quality ART service provision

The concluding statements on participants' perceptions regarding their requirement for improving the quality of ART service provision in public health facilities are:

- deploy, train and re-train additional health care workers to meet increasing number and dynamic expectations of end users in ART sites

- $\quad$ integrate HIVIAIDS into regular health services, end users encouraged to attend regular clinics

- $\quad$ improve supply chain management of laboratory reagents and drugs for opportunistic infections, to ensure availability and use

- $\quad$ reduce end user load in the clinic by reducing frequency of hospital visits by stable end users, through dispensing ARVs that will last 3 months per visit

Users' suggestions for actions to improve the quality of ART services were:

- Deploy, train and re-train additional health care workers to meet increasing number and dynamic expectations of end users in ART sites. 
- Integrate HIVIAIDS into regular health services, end users encouraged to attend regular clinics.

- Improve supply chain management of laboratory reagents and drugs for opportunistic infections, to ensure availability and use.

- $\quad$ Reduce end user load in the clinic by reducing frequency of health facility visits by stable end users, through dispensing ARVs that will last 3 months per visit.

\section{Sub-theme 4.4.1: Deploy, train and re-train additional health care workers to meet increasing number and dynamic expectations of end users in ART sites}

This finding refers to the need to improve human resource capacity (both quantitatively and qualitatively) in public health facilities, in order to meet the changing requirement and expectations of end users. Limited human resources coupled with stressful working conditions can result in overwork and burnout, and finally poor quality of care (Tobi et al 2008:1452). Therefore, building human resource capacity to provide quality care in the public health facilities is a formidable recommendation (Tobi et al 2008:1453). Similar findings were reported by Gill and White (2009:14), Wielawski (2006:6) and Singh and Ham (2006:5)

It emanated from the FGD that participants felt appropriate health authorities should deploy, train and re-train additional health care workers to meet the increasing number and dynamic expectations of end users. Direct comments from the FGD participants are illustrated below, to support this finding:

Deployment of health care workers especially doctors was pointed out:

“...There should be enough doctors at hand who will be seeing the entire patients."

“...One observation here...sometimes when I come here, I meet crowd of people joint together. So, if there is possibility, there's need to adding more doctors."

"...the services [in this health facility] need to be improved. They need more doctors and more clinic days per week, to cope with increasing number of patients." 
The need for health care workers was highlighted to address the needs of the community:

"....we need to increase the nurses and the doctors, because of the higher rate of clients. The hospital management need to increase nurses and doctors to meet the increasing demand for services."

"... we need more hands in that place where they are tracing our files. That place is very important for us, because if we are many, there's [there are] only two people there. If one person didn't come or he comes late, everything stops."

Training of health care workers on sharing of information was highlighted:

“....The other area I'll like to mention is in terms of training. When some of these doctors and nurses are being trained, try to step it down. Because this is the complaint we normally hear from them. When somebody have been trained, instead of going back to the members and tell them what to do, they'll not step down the training. And because the members that did that training a lot of money [spent] to one particular person... Here in our support group,... whenever I go for anywhere, I pass the information down, so that if I'm not here tomorrow, somebody can use the information to work."

Training for all was suggested:

"...they should put more workers in all aspects and give them adequate training. Like you're talking about gatemen ...the gateman should know what everyone is doing and the aim of what we are doing, so we won't be trying to protect the interest of our patients and the gateman will be working against it. So, everyone needs training."

Analysing the need for training was perceived as a possibility:

“...So, let there be an expert that look into that [quality of ART services] and see what advice to give, organising trainings ...for the hospital staff at every medical point [of service]. ...it'll go a long way in helping PLHIV."

The need for man power deployment, training and re-training was prominent in the analysis of the FGD.

\section{Sub-theme 4.4.2: Integrate HIVIAIDS into regular health services, end users encouraged to attend regular clinics}

This finding refers to the need to avoid implementing ART service provision as a vertical programme, but focus on integrating it into the general health services and programmes. It also refers to encouraging end users to mainstream themselves into regular health services and clinics, in order to leverage from other health support 
services. Sustaining seamless integrated care during the whole care process is most reasonable to achieving end user satisfaction (Minkman et al 2007:91; Ding et al 2008:40).

Analysis of FGD indicated that participants were of the view that HIVIAIDS services should be integrated into regular health services, and end users encouraged to attend regular clinics. Illustrated below are direct comments from the FGD participants as evidence hereof.

Service integration reduces stigma and re-inforce end user rapport with their health care workers, especially, doctors:

"...we should remove the stigma aspect of it, where these each patient has their own clinic so that they now know the doctors they are seeing. You can ask some of us here now the name of their doctor, they'll not be able to tell you because doctors changes. Before you know it they have changed from one to another."

"...In pharmacy, we have one pharmacy for everybody, is there any stigma there? $P M M$, everybody go there, is there anything like stigma there? Social welfare we go there for counselling so what stops you from seeing your own personal doctor?"

“...No, no, no, no [everyone in the group wants general integration without special assignment of each client to a doctor]"

"...Using separate clinic you are already stigmatising yourself and you are attracting people to look through."

End users of ART services should attend regular clinics, throughout the week:

"... Let the clinic days be from Monday to Friday, now they only do three times a week. If the clinic day is held from Monday to Friday, it will help in addressing some of these problems [of congestion of patients and long waiting time on clinic days]."

"...they need more doctors and more clinic days per week, to cope with increasing number of patients."

"...if they are giving drugs, they give two weeks ahead of the time you are supposed to come and collect your drugs"

Integration of ART into regular health care services emerged prominently from the analysis of the FGD. End users assert that this will go a long way in improving quality of care. 
Sub-theme 4.4.3: Improve supply chain management of laboratory reagents and drugs for opportunistic infections, to ensure availability and use

This finding relates to strengthening logistics and ARV delivery systems to ensure uninterrupted and efficient supplies at all times (Wielawski 2006:6; Singh \& Ham 2006:5). Similar recommendations were noted in Chiegil (2010:51) and Alemayehu et al (2009:360).

It emanated from the FGD that participants felt supply chain management of laboratory reagents and drugs for opportunistic infections, should be improved to ensure availability and use. The following direct comments from the FGD participants are evidence hereof.

Cost of Ols limits end users' access to OI drugs:

“...Ols should be taken more seriously, so they should consider adding money into Ols"

“...In [my health facility] apart from the ARVs, other drugs we use to pay..."

Resolve laboratory reagent stockout by addressing logistics challenges centrally, at the national level:

"... if reagents at the central [national] level can be sorted out, the [reagent stock out issues] will be Ok [resolved]."

It emanated from the FGD that once logistics for Ols and lab reagents were resolved, the challenges of stock out could be addressed and end users could be satisfied with care.

Sub-theme 4.4.4: Reduce end user load in the clinic by reducing frequency of health facility visits by stable end users, through dispensing ARVs that will last 3 months per visit

This finding relates to the perception that if end users' load can be reduced in the clinics, their satisfaction could be ensured. Thus, participants suggested dispensing enough ARVs to last 3 months per end user, so that frequency of health facility visits will be reduced, thereby reducing end user load in the clinics. In some programmes, end 
users faced inadequate supplies of drugs, which caused dissatisfactions, but could not tell what the cause of drug outages were (Karunamoorthi et al 2009:337; Gutierreze et al 2009:61).

Analysis of FGD indicated that participants were of the opinion that to reduce end user load in the ART clinics, health care authorities and service providers should consider reducing frequency of health facility visits by stable end users. Illustrated below are direct comments from the FGD participants as evidence hereof.

Dispense drugs that will last three months, during end user refill visits:

"... [appropriate authorities] should look into giving 3 months drugs, now we have it 2 monthly. If they give 3 months, I think it'll equally help."

“...if they are giving drugs, they give two weeks ahead of the time you are supposed to come and collect your drugs."

“...For those coming from faraway places, I suggest the hospital dispense drugs for 2-3 months to reduce their frequency of visits to hospital. Aside from new patients who have to come to proof that they can adhere to the medications, all returning patients should receive 2-3months stock of ARVs."

It emanated from the FGD that increasing ARV refill period to three months could reduce the burden of health facility attendance by end users, as well as reduce health care workers' burnout.

\subsection{CONCLUSION}

This chapter presented the findings of the analysis of qualitative data obtained from the six FGDs conducted at the six geopolitical zones of Nigeria among end users attending ART services. The framework approach of data analysis and Weft QDA version 1.0.1 software were used in this study. Through this approach, the researcher used a systematic procedure for analysing the qualitative data. The procedure comprised the following steps: familiarisation, identifying a thematic framework, indexing, charting, mapping and interpretation. Data were presented according to themes and sub-themes, which resulted from the data analysis. Four major themes were identified from the data analysis: One of the themes relate to ART services that met participants' requirement. It emerged from the analysis of FGD that end users were spiritually and physically happier due to frequent availability of drugs and team work among health care workers. It also 
emanated from the FGD that growing enthusiasm reinforce volunteers to commit time and resources to fill human resource gaps, including implementing strategies to promote infection prevention and control in the clinics. Leadership of support groups are knowledgeable and lead dialogue with health authorities to seek greater quality of care, including treating end users with passion and empathy. End users appreciate linkages between health facilities and communities to reinforce seem less continuum of care, including pharmacy services.

The second theme relates to participants' successes in ART service provision. It emerged from the analysis of the FGD that health facilities should develop linkages with other specialised facilities to refer end users or their samples to complete lab tests. Health facilities should also maintain back-up laboratory machines to avoid interruptions in lab services. End users require that internal processes should be continuously improved and end users empowered in order to be retained in care. End users require health facilities to always have adequate clean water, constant electricity, and adequate ventilation; and be aesthetically attractive. End users want to spend the least amount of time when they come to the health facility. Therefore, health facilities should recognise the common cohesion among PLHIV and involve them in decision making regarding plans for improvements in processes and procedures. End users recognise that health care workers fuel stigma and discrimination against PLHIV, and expect that this be contained. Health care workers should continue to provide uninterrupted ARVs and meet end users' expectations for examining their vital signs each time they visit the health facility.

The third theme that emerged from the FGD was ART services that disappointed participants from meeting their requirement. It emanated from the FGD that changes in health facility leadership could cause weak support for support group activities, and thus, reduce end user satisfaction. Health facilities providing ART services are overcrowded beyond their space limits. They also experience constrained storage space, inadequate lighting and unattractive environment. End users pay the price of poor quality caused by inadequate health systems: weak laboratory management systems, inadequate health care workers, excessive burden on equipment and frequent lab equipment breakdown. Others include extortion of money, out of pocket payments for OI drugs and long waiting time. End users also face discrimination, intimidation and 
scolding from health care workers, possibly due to dearth of health care workers and staff burnout; yet, complaints management mechanisms are obscured.

The final theme that emerged from the analysis of FGD relates to suggestions for improving quality of ART services provision. The need for man power deployment, training and re-training, and integration of ART into general health services were prominent in the analysis of the FGD. Ensuring availability of OIs and lab reagents and increasing ARV refill period to three months could reduce the burden of health facility attendance by end users, as well as reduce health care workers' burnout.

The findings regarding end users' perceptions concerning quality of ART service provision in Nigerian public health facilities are summarised in Table 4.3, below, against each element of the Wagner's Chronic Care Model.

Table 4.3 Summary of findings regarding end users' perceptions concerning quality of ART service provision in Nigerian public health facilities

\begin{tabular}{|c|c|c|}
\hline Theme & Findings from the study & $\begin{array}{l}\text { Elements of } \\
\text { Wagner's Chronic } \\
\text { Care Model }\end{array}$ \\
\hline \multirow{6}{*}{$\begin{array}{l}\text { ART services that } \\
\text { met end users' } \\
\text { requirement }\end{array}$} & $\begin{array}{l}\text { - Uninterrupted supply of ARV drugs could improve } \\
\text { quality of life }\end{array}$ & $\begin{array}{l}\text { Delivery system } \\
\text { design }\end{array}$ \\
\hline & $\begin{array}{l}\text { - Courtesy service of doctors, nurses and other health } \\
\text { workers serve as source of encouragement for } \\
\text { PLHIV } \\
\text { - Empathising with the end user while counselling } \\
\text { improves quality of life of the end user } \\
\text { - ART knowledge explosion improves cohesion } \\
\text { among PLHIV and raises demand for quality of care }\end{array}$ & $\begin{array}{l}\text { Self-management } \\
\text { support }\end{array}$ \\
\hline & $\begin{array}{l}\text { - Support group volunteers are enthusiastic of } \\
\text { improving the quality of life of their members } \\
\text { - Support group volunteers promote infection } \\
\text { prevention and control through triage of vulnerable } \\
\text { members in the clinic }\end{array}$ & Decision support \\
\hline & $\begin{array}{l}\text { Volunteer pharmacists improve quality of pharmacy } \\
\text { services in ART clinics }\end{array}$ & $\begin{array}{l}\text { Clinical information } \\
\text { systems }\end{array}$ \\
\hline & $\begin{array}{l}\text { - Strengthening linkages between support groups and } \\
\text { community based organisations provide } \\
\text { opportunities for wrap around services and improves } \\
\text { the quality of life of PLHIV }\end{array}$ & $\begin{array}{l}\text { Community } \\
\text { resources }\end{array}$ \\
\hline & $\begin{array}{l}\text { - Leadership of support group implement innovative } \\
\text { approaches to improve dialogue with health } \\
\text { authorities to improve quality of life of end users } \\
\text { attending ART }\end{array}$ & $\begin{array}{l}\text { Health care } \\
\text { organisation }\end{array}$ \\
\hline
\end{tabular}




\begin{tabular}{|c|c|c|}
\hline Theme & Findings from the study & $\begin{array}{l}\text { Elements of } \\
\text { Wagner's Chronic } \\
\text { Care Model }\end{array}$ \\
\hline \multirow[t]{6}{*}{$\begin{array}{l}\text { Participants' } \\
\text { perceived } \\
\text { successes in ART } \\
\text { service provision }\end{array}$} & $\begin{array}{l}\text { Health facilities are spacious, clean and attractive, } \\
\text { with adequate infrastructure to meet environmental } \\
\text { health standards and the aesthetic needs of end } \\
\text { users } \\
\text { Lifesaving ART programme is fundamental to the } \\
\text { survival of PLHIV, thus, will not experience } \\
\text { interruptions. } \\
\text { Health facilities ensure that any end user who turns } \\
\text { up to the health facility benefits from full assessment } \\
\text { of his current health status; and is counselled on } \\
\text { emerging clinical manifestations that needs medical } \\
\text { attention. }\end{array}$ & $\begin{array}{l}\text { Delivery system } \\
\text { design }\end{array}$ \\
\hline & $\begin{array}{l}\text { - Health facilities recognise the essence of PLHIV as } \\
\text { volunteers, and design capacity building and } \\
\text { motivational programmes to meet both the task } \\
\text { requirement and volunteers' expectations. }\end{array}$ & $\begin{array}{l}\text { Self-management } \\
\text { support }\end{array}$ \\
\hline & $\begin{array}{l}\text { Health facilities providing ART services have } \\
\text { developed linkages with relevant facilities, to obtain } \\
\text { specialised diagnostic services, and accept results } \\
\text { arising from such facilities. }\end{array}$ & Decision support \\
\hline & $\begin{array}{l}\text { Health facilities have standardised processes that } \\
\text { ensure end users spend the least amount of time } \\
\text { seeking health care in the facility }\end{array}$ & $\begin{array}{l}\text { Clinical information } \\
\text { systems }\end{array}$ \\
\hline & $\begin{array}{l}\text { - Government, communities and individuals have } \\
\text { joined forces to ensure a stigma free environment for } \\
\text { PLHIV. } \\
\text { - Health facilities recognise that PLHIV loves } \\
\text { interacting with their peers, and creates } \\
\text { opportunities for such interaction as part of the } \\
\text { continuum of care process. }\end{array}$ & $\begin{array}{l}\text { Community } \\
\text { resources }\end{array}$ \\
\hline & $\begin{array}{l}\text { Health facilities continuously improve quality and } \\
\text { processes to meet the changing requirement and } \\
\text { expectations of end users. }\end{array}$ & $\begin{array}{l}\text { Health care } \\
\text { organisation }\end{array}$ \\
\hline \multirow[t]{4}{*}{$\begin{array}{l}\text { ART services that } \\
\text { disappointed } \\
\text { participants from } \\
\text { meeting their } \\
\text { requirements } \\
\text { (dissatisfaction) }\end{array}$} & $\begin{array}{l}\text { Overstretched, space constrained and aesthetically } \\
\text { non-attractive ART care infrastructure } \\
\text { - Frequently interrupted laboratory services, related to } \\
\text { high equipment down time and commodities stock- } \\
\text { out } \\
\text { - Inadequate supply and availability of drugs for } \\
\text { opportunistic infections and multivitamins }\end{array}$ & $\begin{array}{l}\text { Delivery system } \\
\text { design }\end{array}$ \\
\hline & $\begin{array}{l}\text { Perceived increasing incidences of failure to treat } \\
\text { end users with courtesy, related to inadequate } \\
\text { health care workers and staff burnout of the } \\
\text { available few. }\end{array}$ & $\begin{array}{l}\text { Self-management } \\
\text { support }\end{array}$ \\
\hline & $\begin{array}{l}\text { Weak responsiveness to the needs of end users by } \\
\text { the health facility leadership, policy and strategy }\end{array}$ & Decision support \\
\hline & $\begin{array}{l}\text { Perceived increasing trends of inadequacy in } \\
\text { ensuring end users emerge from the health facility } \\
\text { healthy }\end{array}$ & $\begin{array}{l}\text { Clinical information } \\
\text { systems }\end{array}$ \\
\hline
\end{tabular}




\begin{tabular}{|c|c|c|}
\hline Theme & Findings from the study & $\begin{array}{l}\text { Elements of } \\
\text { Wagner's Chronic } \\
\text { Care Model }\end{array}$ \\
\hline & $\begin{array}{l}\text { - Inadequate processes and procedures for managing } \\
\text { end users' complaints }\end{array}$ & $\begin{array}{l}\text { Community } \\
\text { resources }\end{array}$ \\
\hline & $\begin{array}{l}\text { Sub-optimal standardisation of health care } \\
\text { processes and procedures, with resultant } \\
\text { accumulation of bottlenecks along the workflow }\end{array}$ & $\begin{array}{l}\text { Health care } \\
\text { organisation }\end{array}$ \\
\hline
\end{tabular}

The next chapter (chapter 5) will address the discussion of key findings that were presented in this chapter within the theoretical framework of the Wagner's Chronic Care Model. 


\section{CHAPTER 5}

\section{DISCUSSION OF FINDINGS}

\section{$5.1 \quad$ INTRODUCTION}

In the previous chapter, the research results were presented and this chapter focuses on the discussion of the results. The discussion of research findings is guided by the study objectives described in the first chapter, elements of Wagner's chronic care model, as well as by the themes that emerged from the analysis of focus group discussions. The researcher provided "elaborate/detailed" descriptions of defining attributes, descriptions of what the participants (end users) did and did not discuss in the focus groups, and quotes from the end users that were illustrative of the themes. The researcher used multiple data sources to examine and validate conclusions about meanings. The researcher also provided commentary about the specific new knowledge generated by the study; including activities to be included in the best practice guidelines for meeting end users' requirements for quality ART in public health facilities. The researcher also discussed specific implications of the findings to public health, tying the implications to existing literature, to enhance the credibility of the interpretations of the findings.

\subsubsection{Overview of research discussions}

This study sought to explore and describe the perceptions of end users concerning the quality of ART in Nigerian public health facilities.

In this study, four major themes were identified, namely:

THEME 1: ART services that met participants' requirements

THEME 2: Participants' perceived successes of ART service provision

THEME 3: ART services that disappointed participants from meeting their requirements 
THEME 4: Participants' suggestions for actions to improve quality of ART service provision

These four themes and their sub-themes are interpreted below, and validated using relevant literature and the chronic care model to support the interpretation of the findings.

\subsection{ART SERVICES THAT MET END USERS' REQUIREMENT}

The participants appreciated that their requirement for quality ART service provision was met in nine key areas, signifying the nine sub-themes that relate to this theme. These sub-themes include uninterrupted supply of ARV drugs, and courtesy services of health care workers towards their end users. End users also appreciated the enthusiasm and participation of support group volunteers to support their peers, including promoting infection prevention and control, and improving dialogue with health authorities to ensure improved welfare of members. Increased demand for quality ART service provision received reciprocal engagement of volunteer pharmacists who narrowed human resource gaps and empathy by health care workers during counselling sessions. It also included strengthening linkages between community and public health facilities that advanced opportunities for end users' access to community based services.

This finding suggests that when an attempt is made to coordinate and integrate ART service provision to focus on the total needs of the end user, some aspects of the whole care process will meet their requirement. This conforms to the principle of the CCM which supports an integrated and coordinated care process that is user-focused (Minkman et al 2007:91). Minkman et al (2007:91) elucidate that the CCM describes six elements - the community, the health system within it and four elements with the health system: self-management support, delivery system design, decision support and clinical information systems. In order to achieve ART service provision that met end users' requirement and expectation, ART service provision demonstrated links between the health facility, community and the end users, which are characteristic features of the CCM. Creel et al (2002:1) corroborates that high-quality services ensure that end users receive the care that they deserve. Furthermore, providing better services at reasonable cost attracts more end users, increases the use of health services, and reduces the 
number of medical complications. Several impact studies have shown that improving the quality of health services increases health service uptake (Creel et al 2002:1).

Each of the nine sub-themes of this theme is further discussed below.

\subsubsection{Uninterrupted supply of ARV drugs improve quality of life}

Uninterrupted supply of ARV drugs in public health facilities was one of the findings that met end users' requirements for quality ART service provision. The availability of ARV drugs was evident from the data. Also from the data, a spiritual reason was given for the availability of drug supplies in the health facility. Mentally, clients were happier with themselves due to their receiving drug supplies from the health facilities attended. Physical implications due to the availability of drug supplies were also mentioned in the study. What remained unclear to the researcher was whether ARV drugs were available in the right regimens, since end users would not know about such level of details. All they seemed to be concerned about was, as one of the FGD participants said:

"...for the past almost 72 months that l've been taking the drugs, there has never been a month that l'll come without taking the drug home."

This in its sense, may keep end users satisfied, that at least after travelling such distance to the health facility, they are going back home with drugs, irrespective of whether other services were available or not. This finding relates to delivery systems design of the CCM, which supports delivering effective and uninterrupted services, such as uninterrupted ARV drug supplies. This element of the CCM supports team approach to care, where the commodity logistics personnel, pharmacists and other members of the health care team jointly ensure that end users have continuous access to ARV drugs at all times (Wielawski 2006:6; Singh \& Ham 2006:5). Additionally, Creel et al (2002:3) assert that the end user's perspective of the quality of care emphasises availability of commodities as one of the determinants of satisfaction with care.

\section{Conclusion}

It sprang from this study as said in this sub-theme that uninterrupted supply of ARV drugs in public health facilities met end users' requirements for quality ART service 
provision. In this study, this finding was related to the 'delivery systems design' of the CCM, which promotes strengthening systems for delivering effective and uninterrupted ARV drug supplies to all service sites.

\subsubsection{Courtesy service of doctors, nurses and other health workers serve as source of encouragement for PLHIV}

Participants expressed satisfaction that some doctors, nurses and other health workers treated them with courtesy. The collective team approach by health workers (doctors, nurses and counsellors) came to the fore during data collection. It seemed evident from the data that some health workers went the extra mile to care for clients. Some participants did not have a need for interaction with health workers and for them; it was only a physical requirement to take their drugs. Other participants enjoyed a positive relationship with especially doctors. During the courtesy of health workers, they encouraged clients through communication; and a spiritual connection was made between encouragement of staff and God. Courtesy treatment provided by the health care workers reinforced end users' confidence in the public health facilities. One participant verbalised:

\footnotetext{
“...l enjoy the treatment of doctors. The doctors, they are too good, and the nurses, the people they are working. They are too good. I enjoy the hospital because they take care of me. When I came here, I was very lean; I didn't know I'll be like this now. So, I enjoy their treatment and the drugs given to us, I enjoyed it".
}

Courtesy treatment of health care workers develops confidence and empowers end users' to take on more roles for self-care. In CCM, this element refers to selfmanagement support. This element is described as empowering and preparing end users to manage their health and health care (Wielawski 2006:6; Singh \& Ham 2006:5). Wielawski (2006:6) adds that this principle requires that health care workers should set a tone of collaboration with chronic end users and encourage their participation in setting goals and fine-tuning treatment. Hasin et al (2001:10) report that majority of end users are critical about staff behaviour. End users want to be seen and treated as fellow human beings, as the health workers. No health facility is set out to treat his/her end users without courtesy. Castilo et al (2007:696) maintain that the factor with the greatest predictive power for end user satisfaction was the relationship between the end users and health care workers. Gaioso and Mishima (2007:1) concurred that considerate and 
affective interpersonal relations were one point of satisfaction and one of the aspects that differentiates health care. Hasin et al (2001:7) elucidate the following as examples of treating end users with courtesy: quick response to request for service, intention and attention of health care workers in listening to an end user, diagnosing method, treatment explanation, availability and reception of information.) These were similar to the courtesy treatment demonstrated in this study.

Chiegil (2010:50) reports a study where doctors, nurses and other health care workers were noted to be treating end users with courtesy. It is worthy of note that health facilities that are known to be unfriendly will lose all their end users to competitors. For example, individuals who had received voluntary counselling and testing in Kisesa district in Tanzania resented the need to regularly travel to Mwanza, particularly because the health facility had a reputation that was confusing and unfriendly to villagers (Mshana et al 2006:655).

\section{Conclusion}

It emerged from this study as contained in this sub-theme that some doctors, nurses and other health workers treated end users with courtesy; and their action served as a source of encouragement for PLHIV. In this study, this finding was related to selfmanagement support of the CCM. This element promotes empowering and preparing end users to manage their health and health care.

\subsubsection{Empathising with the end user while counselling improves quality of life of the end user}

Analysis of the FGD revealed that empathising with the end user while counselling improves quality of life of the end user. Participants explained that they feel satisfied and empowered when health workers empathise with them, while counselling. Participants of this study expressed confidence in the quality of counselling services in the health facilities. They also expressed trust in the quality of health care workers providing counselling services in the public health facilities investigated. Putting one's self in the situation of the end user, and talking from the point of view of an informed person makes the end user feel good. This creates an opportunity for the end user to 
feel relaxed and share confidential information and issues of personal concern for guidance and counselling.

When health care workers empathise with end users, they instil confidence and ability 'to do it yourself' into the end users. This in turn, refers to empowering and preparing end users to manage their health and health care (Wielawski 2006:6; Singh \& Ham 2006:5). This in itself is the self-management element of the CCM. The UHCIP (2008:1) admit that modern quality improvement approaches should be adopted for overcoming common barriers to quality of care, understanding and focusing on end user needs. Stenson et al (2005:749) report that 95\% of end users attending ART who participated in their study thought counselling sessions were good, specifying knowledge gained, increased hope and ability to make choices. Kabbash et al (2010:481) on the other hand, report that more than 90 percent of end users in a study in Egypt were satisfied with counsellilng services provided in the health facility. Proctor et al (2006:377) reported from a separate study that end users receiving health care counselling expressed a 99 percent satisfaction rate.

\section{Conclusion}

The study finding featured in this sub-theme that empathising with the end user while counselling improves quality of life of the end user. In this study, this finding relates to self-management support element of the CCM. This element assumes that when health care workers empathise with end users, they instil confidence and ability to do it yourself' into the end users.

\subsubsection{ART knowledge explosion improves cohesion among PLHIV and raises demand for quality of care}

Participants explained during the FGD that ART knowledge explosion improves cohesion among PLHIV and raises demand for quality care. End users want to participate in decisions regarding their care. PLHIV feel more comfortable seeking help from their peers than from anyone else, as cohesion grows among support group members. End users are increasingly demonstrating good understanding of the requirements for their care. It was also evident from the data in this study that the increased knowledge of ART adherence influences end users to detest the notion of 
spiritual healing as cure for AIDS. The escalation of support groups and PLHIV activist movements had caused increasing bonding among members of support groups. Increasing demands for end users' rights to quality health have raised the bar for value associated with health care and put health workers on check for meeting the constantly changing needs of end users. The continuing limitation of health authorities to meeting end users' demands for quality care builds potentials for revolution in the near future. Knowledge explosion is the building block for agitation towards such revolution.

End users use diverse ways to seek knowledge regarding their care, and take initiatives to understand their care. A participant spoke regarding ways by which they seek knowledge in their support group:

"...like the coordinator of our meeting...he is the one, he has concern on us very well. If we have any problems, like something that we don't know, he'll say don't fear to ask questions. Ask questions so that if you ask questions, if he cannot answer us, he'll go and ask the doctor. In our meeting, the doctor doesn't come there, but we shall ask all the questions and he'll take the questions to the doctor."

It is popularly said that 'knowledge is power'. Therefore, increasing knowledge of ART among end users entails empowering and preparing them for taking more responsibility regarding their health, thus, aligning to the self-management support element of the CCM (Wielawski 2006:6; Singh \& Ham 2006:5). End user movements have added to end users' knowledge base for making more educated decisions regarding their health care. End users' perceptions of quality are therefore, a matter of great importance in continuous quality improvement (CQI) activities and efforts. Coulter (2010:4) argues that end users' attitude to the likely benefits and risks of their illness should be a key factor in ensuring an informed choice of treatment. Use of evidence based decision aids has been shown to lead to improvements in end users' knowledge, better understanding of treatment options, more accurate perception of risks, and reduced demand for elective procedures (Coulter 2010:4). Studies have found that end users who report greater involvement in medical care are more satisfied with their health care workers (Beach et al 2007:1119). They also report more understanding, reassurance, and perceived control over their illness, and have improvements in medical conditions. Colvin et al (2003:S86) report that interviews with 41 of 51 end users of traditional healers who had completed tuberculosis treatment in Hlabisa, KwaZulu-Natal, South Africa, revealed high levels of satisfaction with the care received. 


\section{Conclusion}

It stemmed from this study as claimed in this sub-theme that ART knowledge explosion improves cohesion among PLHIV and raises demand for quality care. Increasing knowledge of ART among end users entails empowering and preparing them for taking more responsibility regarding their health. Therefore, this study aligned the finding to the self-management support element of the CCM.

\subsubsection{Enthusiasm of support group volunteers to improve the quality of life of members}

It emanated from the FGD that participants felt support group members express enthusiasm to serve as volunteers to improve the quality of life of their members. End users commit time and resources to learn new experiences in order to help PLHIV maintain their up keep. Volunteers are delineated by geographic convenience to assist support group members living around their communities; as contact tracking increasingly becomes a key role of support group volunteers. On the course of their volunteer work, end users identify and fill human resource gaps in health facilities.

While the interest to volunteer is there, they also share concerns regarding policy environment and external support to facilitate their work, such as payment for travels, commodities, among others.Support group volunteers agitate for a policy environment that supports decision for them to have access to supplement health care workers' efforts to care for their peers in the health facility. Support group members seek opportunities to learn new ways of providing care and support to their members, and make effort to translate knowledge gained into practice. They also make personal efforts to stay in contact with their peers. They identify where each other lives, exchange addresses and conduct home visits to check on the welfare of each other. This helps in contact tracking of peers, when not seen in the health facility over successive periods of time. This provides new opportunities for dealing with defaulter tracking in ART programmes. For example, Torpey, Kabaso, Mutale, Kamanga, Mwango, Simpungwe, Suzuki and Mukadi (2008:1) suggest that the deployment of adherence support workers (ASWs) helped reduce waiting times for adherence counselling. Loss to follow-up rates of new end users declined from $15 \%$ to $0 \%$ after the deployment of ASWs. Wagner et al (2007:871) lament that to minimise end user 
dropping out of care, structures (such as support groups) should be developed to enable end users provide support to each other, and have a "safety net" for helping each other remain in care. In another study conducted to explore the perception of rewards among volunteers working in home-based care settings, Akintola (2010:10) espoused that volunteer caregivers derived intrinsic rewards related to self-growth and personal (emotional and psychological) development on the job. They also derived satisfaction from community members taking a liking for them and expressing a need for their services (Akintola 2010:10). Akintola (2010:10) continued that volunteers felt gratified by the improvements in their health behaviours, which were direct consequences of the experiences of caring for terminally ill end users with AIDS. Extrinsic rewards came from appreciation and recognition shown by end users and community members (Akintola 2010:10). Akintola (2010:10) notes that extrinsic rewards also accrued to volunteers when the services they rendered made their end users happy. Perhaps the greatest sources of extrinsic rewards are skills and competencies acquired from training and experience while caring for their end users, and volunteers' ability to make a difference in the community (Akintola 2010:10). Evidence generated from the work of these support group volunteers will contribute to decision-making regarding the need to recognise and institutionalise their role in the health facility, thus, aligning to the decision support element of the CCM (Wielawski 2006:6; Singh \& Ham 2006:5).

\section{Conclusion}

It emerged from the study as reasoned in this sub-theme that support group members express enthusiasm to serve as volunteers to improve the quality of life of their members. Evidence generated from the work of the support group volunteers will contribute to decision-making regarding the need to recognise and institutionalise the role of support group in the health facility. In this study therefore, this finding aligns to the decision support element of the CCM.

\subsubsection{Support group volunteers promote infection prevention and control through triage of vulnerable members in the clinic}

Participants explained during the FGD that support group volunteers promote infection prevention and control through triage of vulnerable members in the clinic. Participants 
demonstrated understanding of the need to separate persons with highly communicable diseases from others who are vulnerable, as a means of infection prevention and control in health facilities. The volunteers adopted this practice following sensitisation by health workers, that if adequate precaution is not taken, they are very likely to acquire nosocomial infections in the health facility. They learned that separating vulnerable people, such as separating HIV positive end users from those with active TB will reduce the spread of TB among HIV positive end users. Volunteers work in the clinics to educate PLHIV through health talk, and assist health workers to triage end users based on their vulnerability and risk exposures. A participant who is aware of his volunteer role in the health facility verbalised thus:

"...I am a member of the IPAC [infection prevention and control committee]. So, when I go to the meeting, I come and relate anything we discussed there to the support group, even at the clinic. And let them know if they are having TB [tuberculosis], those people that are coughing we use to separate them. We let the doctor see them first, before other people, so that they can go early."

This is an important finding which given sufficient coordination and additional information for decision support, will enhance the application of the CCM in public health facilities. This finding aligns with the CCM element, decision support, and relates to promoting care that is consistent with research evidence and end user preferences (Wielawski 2006:6; Singh \& Ham 2006:5). The use of support group volunteers to promote infection prevention and control is an area requiring policy support, encouragement and promotion, due to its importance in ensuring end user safety and improved quality of ART service. Health care providers must ensure that all end users leave the health care system healthy. This corroborates with the notion by AHRQ (2010:135) that the goal of safety in health care system is that the system is: ...relentless in continually reducing the risks of infection or injury from care, aiming for "zero" harm wherever and whenever possible - a system that can promise absolutely reliable care, guaranteeing that every end user, every time, receives the benefits of care based solidly on science. AHRQ (2010:135) elucidates that the goal of end user safety include:Health care facility and staff ensuring a culture of safety in order to reduce health care-associated infections (HAIs) and serious adverse events (SAEs); health facilities reducing preventable and premature health facility level mortality rates, and health facilities improving their 30-day mortality rates of selected conditions (e.g., pneumonia). 
These goals are attainable, if pursued. For example, following quality improvement initiatives in the US, AHRQ (2010:138) reports that the percentage of adult surgery end users who received appropriate timing of antibiotics improved from 2005 to 2008 (74.9\% to $91.4 \%$ ). Improvements were also seen among all age groups during this period. Also, from 2004 to 2007, the rate of deaths following complications of care declined from 128.9 to 105.7 per 1,000 admissions of adults ages $18-74$.

\section{Conclusion}

It emanated from the study as said in this sub-theme that support group volunteers promote infection prevention and control through triage of vulnerable members in the clinic. For the purpose of this study, this finding aligns to the CCM element, decision support, and relates to promoting care that is consistent with research evidence and end user preferences.

\subsubsection{Volunteer pharmacists improve quality of pharmacy services in ART clinics}

It emerged from this study that participants felt volunteer pharmacists improve the quality of pharmacy services in ART clinics. Participants described activities of volunteer pharmacists as user focused. They supported the health facility pharmacists to close the human resource gap in the pharmacy, thereby, meeting their expectations for quality pharmacy services. The volunteers performed functions such as drug dispensing, recording and documentation of medications dispensed (including adverse drug reactions), updating pharmacy records and sorting and retrieval of pharmacy records. The major thrust of this finding was the ability of the volunteer pharmacists to maintain pharmacy records. This in essence will improve the quality of ART service through availability of clinical information for easy end user data retrieval. This is an aspect of care that is promoted by the CCM, through its element called clinical information systems. Wielawski (2006:6) and Singh and Ham (2006:5) note that this element relates to organising end users data to facilitate efficient and effective care, such as, organising the care team to measure the performance of pharmacy services against best practices or quality benchmarks.

With quality pharmacy services, reviewed literature indicates that end users can be satisfied with services. Barbosa (2011:103) reveal that to improve satisfaction with 
pharmaceutical care there is need for greater attention and educational activities with end users who mostly have low rates of education. Furthermore, dialogue, counselling and clarification on the pharmacotherapy were recommended (Barbosa 2011:103). In another study in Vitoria, Brazil, to evaluate the satisfaction of users of a Pharmaceutical Care (PC) service in a private communitarian pharmacy, Andrade et al (2009:349) lament that a higher level of satisfaction was reported by users of the PC service, with values between three and five. The higher averages for the establishment with PC service were the result of greater perceived pharmacist interest in the end user's health. As the same results were not obtained by the services without $\mathrm{PC}$, it was concluded that this practice is very important to the satisfaction level of users of pharmacy services.

Karunamoorthi et al (2009:331) sought to determine end users' level of satisfaction and their expectations with pharmacy services at specialist ART unit in the government hospitals of Addis Abbaba, Ethiopia. Results of the study as reported by Karunamoorthi et al (2009:337) showed that end users were dissatisfied with the pharmacy services. Major area of dissatisfaction was long waiting time in the pharmacy, which gulped $82,5 \%$ of respondents investigated. Some of them complained about lack of information on ARV drugs dispensed to them - most (72,6\%) wanted both written and oral information/instructions regarding the drugs provided. End users $(22,4 \%)$ also said they were dissatisfied because the pharmacists were impolite towards them.

\section{Conclusion}

It emerged from the finding as maintained in this sub-theme that volunteer pharmacists improve the quality of pharmacy services in ART clinics. In this study, this element will be referred to as clinical information systems, which relates to organising end users data to facilitate efficient and effective care, such as, organising the care team to measure the performance of pharmacy services against best practices or quality benchmarks. 


\subsubsection{Strengthening linkages between support groups and community based organisations provide opportunities for wrap around services and improves the quality of life of PLHIV}

Analysis of the FGD indicated that participants were of the view that strengthening linkages between support groups and community based organisations provide opportunities for wrap around services and improves the quality of life of PLHIV. Linkages with community based organisations were mentioned by study participants. Community pharmacies established linkages with public health facilities to support continuum of care for PLHIV. Since it is not practicable for one organisation or facility to provide for the total needs of end users, it was fundamental for the de facto health facility to strengthen partnerships/linkages with sister organisations, in both the public and private sector, to refer end users who need specialised facility care or community based services such as home based care, income generating activities, food and nutrition services, and much more. This was corroborated by Minkman et al (2007:91) who, in advancing the benefits of the CCM, said it is important to sustain seamless integrated care during the whole care process. Adding that for health facilities, this requires horizontal coordination, collaboration with other organisations and community partners or service integration. Partners in the care chain and the functioning of the care chain or care network as a whole contribute to the quality of care (Minkman et al 2007:91). In the CCM, the element that supports this finding is referred to as community resources. This element relates to identifying and mobilising community resources to meet the needs of end users with long-term conditions such as HIVIAIDS (Wielawski 2006:6; Singh \& Ham 2006:5).

When the community and health facility work together, health care quality will be improved. The UHCIP (2008:1) corroborates that engaging teams of managers, service providers, and community stakeholders is one of the principles for modern quality improvement and an approach for overcoming common barriers to quality of care. Creel et al (2002:7) also explained that improving quality of care for end users requires understanding their cultural values, previous experiences, and perceptions of the role of the health system, and then bringing health service providers and the community together to map out a shared vision of quality. This speaks positively to strengthening linkages between health facilities and community programmes to meet end users' requirements and expectations. To maximise impact on social and economic health, 
Wagner (2007:871) recommends that it is important for ART programmes to develop effective mechanisms and linkages for coordinating and referring end users to support service organisations in the community. Better community support for health care workers and for end users on chronic illnesses is also perennial themes in end user surveys (Coulter 2005:1200). These groups require well cooordinated local services rather than a diversity of competing health care workers or health care facilities (Coulter 2005:1200).

\section{Conclusion}

The finding of the study revealed in this sub-theme that strengthening linkages between support groups and community based organisations provide opportunities for wrap around services and improves the quality of life of PLHIV. In the CCM, the element that supports this finding is referred to as community resources. This element relates to identifying and mobilising community resources to meet the needs and expectations of end users with long-term conditions such as HIVIAIDS.

\subsubsection{Leadership of support group implements innovative approaches to improve dialogue with health authorities to improve quality of life of people on ART}

Participants explained during the FGD that the leadership of support group develop and implement innovative approaches aimed at improving dialogue with health authorities. Leadership of support groups mediate between health authorities and their members. Leadership of support groups also monitor implementation of resolutions/action points reached with health authorities, and substantiate dialogue where issues are identified. The dialogue aims at improving relationships with health authorities and to address the requirement and expectations of their members. In some cases, the dialogue yielded desired results of meeting end users' expectations in the health facility. An example in the words of the participant includes:

"...many of the nurses that use be ruling us bad, due to this complain all the time, many of them have changed their attitude. They have changed their attitude. Like that matron upstairs, she now behaves better, all this kind of shouting, and screaming and driving people aside. She has now come to realise that her job is also on edge because of the too much complaints on her." 
In CCM, this finding was seen to relate to health care organisation or health system. This element relates to creating a culture, organisation, and mechanism that promote safe, high quality care, including making excellence a priority and pursuing it visibly (Wielawski 2006:6; Singh \& Ham 2006:5). Essential to achieving this, Wielawski (2006:6) said top management support and open communication or error and failings as well as strategies for improvement must be put in place. In line with this expectation, this finding elaborated that leaders of the support group of PLHIV, representing the end users identified and filed complaints to health facility leadership, on areas contributing to dissatisfaction among their members. The health facility leadership in turn, was supportive and used open communication in addressing the errors in their system.

\section{Conclusion}

It emanated from the study as argued in this sub-theme that the leadership of support groups develop and implement innovative approaches aimed at improving dialogue with health authorities.The dialogue was aimed at improving relationships with health authorities and to address the requirement and expectations of their members. In CCM, this finding was seen to relate to health care organisation or health system. This element relates to creating a culture, organisation, and mechanism that promote safe, high quality ART service, including making excellence in ART service provision a priority and pursuing it visibly.

\subsection{THEME 2: PARTICIPANTS' PERCEIVED SUCCESSES IN ANTIRETROVIRAL THERAPY SERVICE PROVISION}

This theme relates to participants' health care experiences that they perceived as successes in ART service provision. End users perceive that health facilities providing ART services should be linked to other specialised health and related services, implement continuous quality/process improvement (CQI/CPI), including ensuring adequate aesthetics in the facility. Other perceptions include timeliness of services, stigma reduction, and ensuring uninterrupted supply of ARVs. End users also expect regular health assessment and counselling, and recognition of PLHIV volunteer scheme and support group activities in the health facility. This multidimensional of end users corresponds with current definitions of the quality of care itself. The Institute of Medicine (IOM), in Minkman et al (2007:90) defines good care as safe, effective, timely, end user 
centred and efficient. This definition also reflects multiple dimensions of quality, including organisational aspects like streamlined care process, adequate space and aesthetics and a financially healthy system that ensures uninterrupted ARV drugs supplies.

Based on the multidimensional requirements arising from this study, only an integrated quality management model such as the CCM can address end user requirements, all at once. Wouters et al (2008:2) and Liu and Wang (2007:266) report that end user satisfaction has become an important performance measure and outcome of health care. Porzsolt et al (2007:2) affirm that ten minutes of a health care worker's full attention and the perception of empathy are much more valuable to end users than twenty business minutes of the health care worker's time budget. Thus, placing the responsibility for determining the quality of ART service provision in the hands of end users, rather than health care workers whose traditional health care view of technical quality is inadequate to manage this complex (Gill \& White 2009:14). Minkman et al (2007:91) provided sufficient justification for using the CCM in chronic conditions, such as in ART service provision. Firstly, Minkman et al (2007:91) elaborated that the CCM consist of multiple 'enablers' of good quality care (for instance, leadership or delivery system design) that once implemented, will improve quality ART service.

The enablers have characteristic features that address all health care processes, structures and mean values of the health facility. Secondly, this model has the features to focus on multiple dimensions/expectations of end users at the same time (for instance, CQI can be implemented alongside with ensuring aesthetics, while clinical information generated from pharmacy services will be used to track and improve drug supplies to ensure uninterrupted supplies). Lastly, the CCM assumes dynamic relationships between improved performance and implementation of interventions based on the model's enablers (Minkman et al 2007:91). For instance, in a CQI activity to meet end users' expectations, keeping the quality standards consistently high requires continuous cycles of participatory planning, diagnosis, implementation, evaluation/user feedback and over again to planning, and so on.

Creel et al (2002:2) note that quality of care is a multi-dimensional issue that may be measured constantly but differently, depending on end users'priorities. In the current study, we examine the end users' requirement as their priorities, although they may be 
influenced by social and cultural concerns, and place significant emphasis on the human aspects of care. Hasin et al (2001:6) suggest that the main factors, which affect end user satisfaction, are: doctor, drug, diagnosis, duration, distance, affordability and prompt service. Adding that these are the factors that comprise the health care quality system, and the higher the efficiency of the quality system, the more will be the satisfaction of end users. In this study, much more factors were identified to affect end user satisfaction.

Each of the nine sub-themes of this theme is discussed in more detail, below.

\subsubsection{Health facilities are spacious, clean and attractive, with adequate infrastructure to meet environmental health standards and the aesthetic needs of end users}

It emerged from the FGD that the participants perceive health facilities to be spacious, clean and attractive, with adequate infrastructure that was meeting the environmental health standards and the aesthetic needs of end users. Consistent availability of clean water supply in health facilities came to the fore in the data collected. Others include constant electricity supply and adequate ventilation of health care settings. Participants of the study noted that the health care setting should be comfortable for end users to feel relaxed during health facility visits. It should also be aesthetically attractive, to give a home like feeling to end users.

The availability of adequate space, in addition to the attraction derivable from it, helps in improving ventilation and addressing infection prevention and control in the ART clinics. Adequate infrastructure, including water and electricity should be readily available, to support end user conveniences. These include water to drink, wash hands and equipment and to flush toilets after use. Others include electricity to power electrical appliances, such as fans, air conditioners, computers, and adequate lighting of the workspace.

Improving infrastructure and overall aesthetics of the work environment does not necessarily require breaking walls and rebuilding. It may just require delivery system redesign to re-align work processes to the available space. It also involves coordinating with the care team to follow up with end users, so that not all have to be at the clinic at 
once, when they do not have to, thus, reducing clinic overload. This principle relates to the CCM element called delivery system design.

This element relates to delivering effective, coordinated, uninterrupted and efficient care in an environment that meets required standards of care (Wielawski 2006:6 and Singh and Ham 2006:5). Pietrovsk and Dall'Agnol (2006:630) indicated aesthetics and cleanliness of the environment as factors that interfere with end user satisfaction. Gaioso and Mishima (2007:1) on the other hand, noted that dissatisfaction in their own study was predominated in terms of environment and organisational accessibility, impairing care longitudinality and continuity. Medeiros et al (2010:402) conducted a study to evaluate end users' perception of a BHU in a medium-sized Brazilian city, analysing their satisfaction and participation in social control.

Results of the study indicated poor infrastructure (physical area) in the BHU (Medeiros et al 2010:402). In another study to compare the quality of family planning services delivered in public and private health facilities in Kenya, Agha and Do (2009:87) report that private facilities were superior to public sector facilities in terms of physical infrastructure and the availability of services. Hasin et al (2001:1) elucidate that frequency of cleaning and time schedule should be adjusted in health facilities. Adding that job environment should be addressed to increase enthusiasm in work. Another study by Chiegil (2010:50) reports that the factors that keep health service workers satisfied with care include keeping ART service environment clean and attractive.

\section{Conclusion}

It emerged from the study as discussed in this sub-theme that end users perceive that health facilities should be spacious, clean and attractive, with adequate infrastructure that meet environmental health standards and the aesthetic needs of end users. This finding relates to the CCM element called delivery system design. This element relates to delivering effective, coordinated, uninterrupted and efficient care in an environment that is designed and accredited to meet acceptable standards of ART care. 


\subsubsection{Lifesaving ART programme is fundamental to the survival of PLHIV, thus, will not experience interruptions}

Analysis of the FGD showed that participants perceive that the on-going free lifesaving ART programme put together by the government of Nigeria in partnership with foreign aid is fundamental to the survival of PLHIV in Nigeria. Therefore, it should not be allowed to experience service interruptions or complete seizure. End users expressed gratitude to God and health care workers for granting them access to the ART programme. Most beneficiaries of the ART service will not afford to pay for services, should fees be introduced in the programme.

The CCM element that relates to meeting the expectations of end users for ensuring no interruptions in ARV drug supplies is the delivery system design. This element relates to strengthening logistics and ARV delivery systems to ensure uninterrupted and efficient supplies at all times (Wielawski 2006:6; Singh \& Ham 2006:5). This element also encourages the health care team to work as a team to forecast, quantify, ensure adequate deliveries and manage the ARV drugs supply pipeline in the public health facilities. Participants for this study were all from public health facilities, thus, could not share opinions from the perspectives of private health facilities. However, Ramchandani, Mehta, Saple, Vaidya, Pandy, Vandrevu, Rajasekaran, Bhatia, Chowdhary, Bollinger and Gupta (2007:140) report lack of end users' confidence in utilising public health facilities, due to inadequate quality of ART services.

Results of their study support previous reports that most newly diagnosed HIV persons enter the health care system via the private health sector. Less than 25 per cent of persons in their study were taking ART, and the vast majority of them (81\%) received their HIV care from private clinics. Surveys of private clinic attendees have found that persons prefer the private sector because there is more flexible and increased access, shorter waiting time, greater confidentiality, and greater sensitivity to user needs (Ramchandani et al 2007:140). However, poor quality of care has also been documented among private providers, particularly in the treatment of infectious diseases such as tuberculosis and malaria, where overuse and inappropriate prescribing has been demonstrated (Ramchandani et al 2007:140). 
Miller et al (2002:598) reported in their findings from California that end users were willing to compromise items that may impact quality of life (i.e., increased side-effects, inconvenience, and pill burden) to have a potent antiretroviral regimen. End users also preferred regimens with fewer side-effects to those with less inconviniences. Pill burden was found to be of relatively low importance for end users than the fact that ARVs are life saving medications (Miller et al 2002:598). In another study in Zambia, Schumaker and Bond (2008:2130) elucidate that end users saw the side effects of ARVs as evidence of their strength and compared these to the side effects of septrin, TB drugs and fansidar (the latter until recently an effective malaria medication commonly used in Zambia). Ananworanich et al (2008:329) found that more end users preferred continuous HAART than CD4-guided HAART. The main reasons for not choosing CD4guided HAART were fear of developing HIV-related illnesses and fear of resistance, indicating that they would not want to notice interruptions in ART services.

\section{Conclusion}

The study finding featured in this sub-theme showed that end users expect that the lifesaving ART programme is fundamental to the survival of PLHIV, therefore, will not be allowed to experience service interruptions or complete seizure. The CCM element that relates to meeting the expectations of end users for ensuring no interruptions in ARV drug supplies is the delivery system design. This element relates to strengthening ARV logistics and supply chain management to ensure uninterrupted and efficient ARV supplies at all times.

\subsubsection{Health facilities ensure that any end user who turns up to the health facility benefits from full assessment of his/her current health status; and is counselled on emerging clinical manifestations that need medical attention}

It emanated from the FGD that participants perceive health facilities to ensure that any end user who turns up to the health facility benefit from full assessment of his/her current health status; and is counselled on emerging clinical manifestations that need medical attention. End users insist on having their current health status examined by health care workers each time they come to the clinic. End users perceive that when a nurse fails to examine their current health status properly, they will proceed to requesting a doctor to do so for them. End users are concerned that if their health status 
is not consistently and adequately examined, their health condition may deteriorate into a situation that could have been avoided. End users want to know the changes taking place in their body, as noticeable through vital signs and related measurements of their body systems. A participant lamented in her words, her requirement and expectation for health care workers to assess the state of her body functions. Thus:

\footnotetext{
"...I want to check my weight, they said you are already too fat, don't break the scale, all this kind of something. Nobody is interested in checking my BP, except when I insisted, 'I want to check my BP!' 'I want to check my BP!!' ... doctor will now be asking me what is wrong with you ... all those kind of things. I am expecting such kind of [checking my health status as reason] for... coming for check-up ... but most times when we come for checkup, it's just to pick up our drugs."
}

This is another factor that only requires service delivery system re-design, which in CCM terms, is referred to as delivery system design. End users expect to be assessed to determine the state of their health. Using the CCM as framework in this context will require reviewing and re-aligning workstations and/or end user appointments in order to cope with their increasing number, while also meeting their expectations for quality ART service provision. This element therefore, relates to strengthening internal systems for ensuring that all end users are routinely provided with basic assessments whenever they turn up for care in the health facility (Wielawski 2006:6; Singh \& Ham 2006:5). Coulter (2005:1199) elucidates that end users want an explanation of their symptoms, treatment, or investigation. Many have their own ideas about what is wrong and what may have caused it, but they do not always articulate these.

This is important against the background that end user centeredness is a core component of quality health care (AHRQ 2010:153). AHRQ (2010:153) defines end user centeredness as health care that establishes a partnership among practitioners, end users, and their families (when appropriate) to ensure that decisions respect end users' wants, needs, and preferences and that end users have the education and support they need to make decisions and participate in their own care. Coulter (2010:4) views shared decision-making as a process in which end users are encouraged to participate in selecting appropriate treatment or management options.

Coulter (2005:1201) elucidates that failures in communication of information about illness and treatment are the most frequent source of end user dissatisfaction. In order to communicate factually and appropriately with the end user, precise examination of 
the end user's health problems and comprehensive treatment plans must be developed (Rad et al 2009:377). Coulter (2005:1200) hypothesise that knowing about current clinical manifestations, the various treatment options available and having a say in these is more important to most end users than having a choice of where to be treated. Hasin et al (2001:10) report that doctors and nurses should pay more attention and take care of end users.

Currently, the end users are left alone and not constantly assessed and reported for a long time, which they do not like. Hasin et al (2001:10) add that end users expect to be closely monitored, procedures and services explained and information provided in simple words. They expect that suitable diagnosing time should be managed; regular and timely visiting schedule for doctors in the ward should be arranged. They also expect that doctors and nurses pay more attention to understand their (end users') minds and be interested in them (end users). Doctors and nurses should ask the end users about their symptoms, because some end users shy away (Hasin et al 2001:10).

HCIP (2008:1) alerts that the search for quality care is guided by its definition that quality health care is an accessible care that is delivered in compliance with evidencebased standards and that addresses end users' needs. High quality care is a function of the health system's ability to assure a continuum of care that addresses end users' needs in an effective, responsive, and respectful manner. To establish evidence based standards, one must rely on measurements of body systems, as obtainable through assessment of end users. Added to evidence based standards is to be responsive to end users' request for assessments to be carried out on them, whenever they visit the health facility.

\section{Conclusion}

It emanated from the study as argued in this sub-theme that participants perceive health facilities to ensure that any end user who turns up to the health facility benefit from full assessment of his/her current health status; and is counselled on emerging clinical manifestations that need medical attention. In CCM this finding may require reviewing and re-aligning workstations and/or end user appointments in order to cope with the increases in their number, while also meeting their expectations for quality ART service provision (delivery system design). This element therefore, relates to strengthening 
internal systems for ensuring that all end users are routinely provided with basic assessments whenever they turn up for care in the health facility.

\subsubsection{Health facilities recognise the essence of PLHIV as volunteers, and design capacity building and motivational programmes to meet both the task requirement and volunteers' expectations}

Analysis of FGD highlighted that participants expect health facilities to recognise the essence of PLHIV as volunteers, and design capacity building and motivational programmes to meet both the task requirements and volunteers' requirements. Volunteers could be motivated through capacity building trainings that will empower them to make some income for themselves. Volunteers could be enlisted as potential employees, pending any opening for employment. Support group needs facilitation and logistics support to reach out to PLHIV through follow up and contact tracking. Programmes working on HIVIAIDS projects could book appointments for, and introduce support groups of PLHIV to key stakeholders.

Activities related to this finding are described as self-management support, as an element of the CMM. This element relates to empowering and preparing end users to manage their health and health care (Wielawski 2006:6; Singh \& Ham 2006:5). In a study conducted to explore the perception of motivation and rewards for volunteers in health care, Akintola (2010:10) espoused that volunteer caregivers derived intrinsic rewards related to self-growth and personal (emotional and psychological) development on the job. They also derived satisfaction from community members taking a liking for them and expressing a need for their services (Akintola 2010:10). Akintola (2010:10) continued that volunteers felt gratified by the improvements in their health behaviours, which were direct consequences of the experiences of caring for end users with AIDS.

Extrinsic rewards came from appreciation and recognition shown by end users and community members (Akintola 2010:10). Akintola (2010:10) notes that extrinsic rewards also accrued to volunteers when the services they rendered made their end users happy. Perhaps the greatest sources of extrinsic rewards are skills and competencies acquired from training and experience while caring for their end users, and volunteers' ability to make a difference in the community (Akintola 2010:10). Wielawski (2006:6) 
adds that support group of PLHIV set a tone of collaboration with chronic end users and encourage their participation in setting goals and fine-tuning their own treatment.

Self-management support empowers end users to take substantial decisions and personal actions regarding their care. Studies have found that end users who report greater involvement in medical care are more satisfied with their health care workers (Beach et al 2007:1119). They also report more understanding, reassurance, and perceived control over their illness, and have improvements in medical conditions. Another study found that end users who prefer a more active role are less satisfied when their physicians do not support their preference (Beach et al 2007:1119).

Chi et al (2004:685) assert that while ART helps volunteer workers to regain capacity to work, other economic supports are needed to enable them and their households to reestablish their livelihoods, especially in resource-constrained settings. It is driven by the notion that no one knows the problems in a process better than those who work (health care workers) and live with the process (PLHIV) on a daily basis. Every problem is treated as a defect in the system, and must be eliminated.

It aims for long-term solutions to problems by seeking to eliminate the root cause of the problems rather than quick fixes. It is the empowerment of workers and volunteers. Everyone is involved in and affected by the transformation; physicians, nurses, volunteers, etc. Stenson et al (2005:952) note that end users must be empowered to recognise and accept quality of care. End users must feel able to communicate freely. Trust and willingness to participate are the foundation upon which the objectives of volunteer work can be achieved. The first step in empowering end users to care for their health is equipping them with information. For example, Torpey et al (2008:1) suggest that the deployment of ASWs helped reduce waiting times for adherence counselling. Loss to follow-up rates of new end users declined from $15 \%$ to $0 \%$ after the deployment of ASWs. Thus, shifting of tasks from more skilled HCWs to ASWs does not compromise the quality of counselling provided. In a resource-limited setting where staff shortages are common, ASWs provide the much needed support for an overburdened workforce. Training of lay providers like ASWs is necessary to help address issues of inadequate human resource associated with ART scale up (Torpey et al 2008:1). 


\section{Conclusion}

Analysis of the study highlighted in this sub-theme showed that end users expect health facilities to recognise the essence of PLHIV as volunteers, and design capacity building and motivational programmes to meet both the task requirements and volunteers' expectations. Activities related to this finding are described as self-management support, as an element of the CMM. This element relates to empowering and preparing end users to manage their health and health care.

\subsubsection{Health facilities providing ART services have developed linkages with relevant facilities, to obtain specialised diagnostic services, and accept results arising from such facilities}

Participants explained during the FGD that they expect health facilities providing ART services to have developed linkages with relevant facilities, to obtain specialised diagnostic services, and accept results arising from such facilities. End users perceive that public health facilities should accept lab results from every reputable laboratory accessible to end users. All health facilities in turn, require back-up laboratory machines to avoid frequent interruptions in laboratory services. These perceptions might have come against the background that sometimes, end users come to the health facility and find no ART lab services available. When they go and carry out tests from other facilities, the de facto health facility will reject the test results without giving them any reasonable explanation for such rejection. In view of the centrality and importance of lab services in an ART programme therefore, end users shared expectations that will ensure they benefit from uninterrupted lab and overall ART services. A participant lamented in his own words:

\footnotetext{
"...I had to go at Teaching Hospital to go and do the test. When I did it there and bring the result, the doctor rejected it, and said that he'll not accept the result."
}

Decision support element of the CCM requires that to link treatment with evidence, health care workers must have explicit guidelines on how to access specialised diagnostic services from other places, address laboratory results coming from outside their immediate facility or refer end users to facilities that have better capacity than theirs. This element also relates to promoting health care practice that is consistent with evidence and end user preferences (Wielawski 2006:6; Singh \& Ham 2006:5). Coulter 
(2010:2) report that end users could choose where they were referred to for laboratory tests, or choose the specialist diagnostic team that will provide their laboratory tests, if they so wish. Hasin et al (2001:10) report that end users like to consult with more specialist health professionals when they come out to seek health care. If such professional (or equipment to deliver such professional service) is not available at any given time, the end user should be referred to where he/she can access such service. What is important is to try not to miss the opportunity of the end users' interest to access such service during the time of visit. Hasin et al (2001:10) emphasised that follow up for complicated service is required. For the end users who need several special tests in several departments, such as operating room, x-ray, etc., (if such services are available within range) a nurse should follow all the steps with test results. Finally, the nurse should combine all and prepare the document when the end user is discharged.

\section{Conclusion}

The study finding featured in this sub-theme revealed that end users perceive health facilities providing ART services to have developed linkages with relevant facilities, to obtain specialised diagnostic services, and accept results arising from such facilities. Decision support element of the CCM requires that to link treatment with evidence, health care workers must have explicit guidelines on how to access specialised diagnostic services from other places, address laboratory results coming from outside their immediate facility or refer end users to facilities that have better capacity than theirs.

\subsubsection{Health facilities have standardised processes that ensures end users spend the least amount of time seeking health care in the facility}

It emerged from the FGD that participants perceive health facilities to have standardised processes that ensure end users spend the least amount of time seeking care in the health facility. Target process improvement measures in the pharmacy and medical records departments to remove long queues and reduce end user waiting time. End users contribute in causing long waiting time; therefore, they should be involved in developing initiatives to reduce the long waiting time. When processes are well laid out, end users use less effort to work through the process within limited amount of time. The 
interest of each end user is to come to the health facility and spend the least amount of time to access services. Immediately thereafter, the end user wants to get back home to continue with his/her aides to daily living or regular job. Process improvement is therefore fundamental in ensuring quality health services.

Timeliness is a challenging end user requirement to attain in public health facilities, where long waiting times have negatively affected satisfaction ratings (Chow et al 2009:438, Rad et al 2009:377, Chavez de Paz et al 2009:22). In a study in Brazil, Oliveira et al (2006:731) report that the mean waiting time for appointment was 94,6 minutes and end users were satisfied with timeliness in this health facility. Another study in Bangladesh revealed that a reduction of waiting time (on average to 30 minutes) was more important to end users than a prolongation of the quite short (from medical standpoint) consultation time (on average 2 minutes, 22 seconds), with 75 percent of end users being satisfied (Aldana et al 2001:512).

In northwest Parana State, Muller and Greco (2010:925) report that the end users were happy with the time taken to set appointments and the time taken for consultations in the health facility. In a study conducted in Pernambuco State, Brazil, Gouvela et al (2011:1849) reveal that the waiting time for health care and the availability of medicines were the factors with the lowest percentage of users' satisfaction. In South of Brazil, Benazzi et al (2010:861) reveal that 75,4 percent pointed deficiencies related to the time they had to wait in the place to the duration of the consultation.

In Nigeria, Udoh et al (2011:222) show that obstetric sonography end users were moderately dissatisfied with the waiting time in both public and missionary hospitals. Several other studies showed dissatisfactions in waiting time (Gutierreze et al 2009:61; Muza \& Muza 2008:12; Lima et al 2007:12; Gaioso \& Mishima 2007:1). Karunamoorthi et al (2009:331) reported that major area of dissatisfaction in their Ethiopia study finding was long waiting time in the pharmacy, which gulped $82,5 \%$ of respondents investigated. Similar finding came out from the study reported by Mfinanaga et al (2008:4) who stated that about $15,0 \%$ to $34,8 \%$ of end users in Tanzania were not satisfied with waiting time, among others. This confirms the importance of time recording and analysis in the chronic care of end users attending ART services. 
A system for measurement, such as time series analysis in CCM is referred to as clinical information systems, which is one of the elements of CCM. Wielawski (2006:6) and Singh and Ham (2006:5) note that this element relates to organising a management information system that helps to achieve timely, efficient and effective care. The CCM is therefore well positioned to addressing timeliness, simultaneously with other aspects of chronic care. According to Creel et al (2002:4), the client's perspective of the quality of care emphasises among others, convenient hours and acceptable waiting times in the health facility. This shows that the promptness and the degree of accessibility of end user records are critical to the successful implementation of TQM, and reduction in end user waiting time. Hasin et al's (2001:1) study determined the elements of end user satisfaction and report that end users expect promptness of health workers to be increased while keeping the health care workers at the current level.

To determine whether timeliness in ART service provision is attainable, AHRQ (2010:148) corroborated that from 2002 to 2007, the overall percentage of adults who needed care right away for an illness, injury, or condition in the last 12 months who sometimes or never got care as soon as wanted decreased (from $16.8 \%$ to $15.3 \%$ ). The AHRQ (2010:147) itemised the following as benefits of timeliness to the health care system: Lack of timeliness can result in emotional distress, physical harm, and higher treatment costs for end users. Timely delivery of appropriate care can help reduce mortality and morbidity for chronic conditions, such as HIVIAIDS. Timely antibiotic treatments are associated with improved clinical outcomes. Early care for co-morbid conditions has been shown to reduce hospitalisation rates and costs for medical care.

\section{Conclusion}

It emerged from this study as argued in this sub-theme that end users perceive health facilities to have standardised processes that ensure end users spend the least amount of time seeking care in the health facility. A system for time measurement or time series analysis in CCM is referred to as clinical information systems, which is one of the elements of CCM. This element relates to organising a management information system that helps to achieve timely, efficient and effective ART care. 


\subsubsection{Government, communities and individuals have ensured a stigma free environment for PLHIV}

Analysis of FGD espoused that participants expect government, health facilities, communities and individuals to have ensured a stigma free environment for PLHIV. Government's support for stigma reduction campaigns could reduce stigma. Despite programmes and activities aimed at stigma reduction, some people (including employers and landlords) remain adamant to change. It also came to the fore of this study that health care workers contribute to exposing end users to stigma. The current health care infrastructure is not well designed to promote confidentiality, and stigma reduction. It was also evident from the study that end users face stigma and discrimination even where they hold support group meetings. Health care workers must be in the forefront of making PLHIV feel free from discrimination and stigmatisation, and become vanguards for change in the community, family and individuals. Until attitudinal change towards setting a stigma free environment begins from the health facilities, it will be challenging to expect the community and individuals to adapt to such environment.

A participant laments that the health care workers themselves are in the forefront of promoting stigma and discrimination in the society. She cites an example of some health care workers who do not maintain confidentiality of their end users' information, thus:

“...I have received series of complaints from my support group members and I reported to the Hospital Secretary. There are some health care workers who say this or that person is also HIV positive. This to me is not appropriate, as there is no confidentiality in care."

Once end users realise that their confidential information has been disclosed by health care workers themselves, they lose confidence in the health facility and withdraw from attending services in such health facility. Therefore, participants call on the government, communities and individuals to join forces and fight stigma and discrimination, to maintain positive health and treat end users with dignity, to prevent further infection cycles.

The CCM has a characteristic feature of removing organisational boundaries in addressing the total requirement and expectations of the end user (Minkman et al 2007:91). This entails that all hands between the government, community and health 
facilities, must be on deck to ensure the total needs of the end user (including stigma and discrimination) are met. These activities contribute to the community resources element of the CCM. Wielawski (2006:6) related this element of CCM to mobilising community resources to meet the needs of people with long-term conditions such as PLHIV. The community is the base upon which the health system and four other elements of the CCM are housed (Minkman et al 2007:91). Fear about disclosure of HIV status and the associated stigma and discrimination is a recurring issue in most studies.

For example a South African based study by Ginwalla et al (2002:707) revealed that end users raised concerns about confidentiality of their HIV statuses, and indeed, their health care in general. Another South African study by Myburgh et al (2005:473) revealed that both race and socioeconomic status were significant predictors of level of satisfaction with the services of the health care workers, after adjusting for gender, age, and type of facility visited. Myburgh et al (2005:473) elucidate that White and high socioeconomic respondents were about 1.5 times more likely to report excellent services compared with Black and low socioeconomic respondents, respectively.

Coulter (2005:1200) canvases for a well coordinated and better community support and continuity of care, to ensure end user safety and a stigma free environment from health facilities to the communities. Coulter (2006:1200) also supports the urgent need to improve support for self care and self management, to build end user confidence and reduce stigma. Liu and Wang (2007:266) re-iterate that end users who are satisfied with health care have a greater sense of confidence and safety during their entire stay in the health facility.

According to Creel et al (2002:3) the end user's perspective of quality of care emphasises respectful and friendly treatment, privacy and confidentiality, service providers' professional competence, information and counselling, convenient hours and acceptable waiting times, and affordability. Three elements can help end users feel well treated: face-to-face communication; skilful providers who show clients that they care about their work; and consideration of how end users' needs, fears, and reactions may be perceived differently by health care providers. To be able to reduce stigma, the health care providers must themselves, adopt behaviours that will not make end users feel stigmatised. For example, where health care providers themselves fail to accept HIV tests, it is conceivable that these nurses, midwives, and physicians may find it hard 
to convince others to undergo a test that they themselves have refused (Chi et al 2004:689). Chi et al (2004:689) report that while attitudes toward HIV may be a barrier to the identification of HIV-positive obstetric end users, provider perceptions about ART medications were overwhelmingly positive. Study findings by Chiegil (2010:51) also revealed socio-economic empowerment and stigma reduction programs for PLHIV as quality of care factors.

\section{Conclusion}

Analysis of the study espoused in this sub-theme that end users perceive government, health facilities, communities and individuals to have ensured a stigma free environment for PLHIV. The community is the base upon which the health system and four other elements of the CCM are housed.Therefore, this finding relates to community resources element of the CCM. The CCM has a characteristic feature of removing organisational boundaries in addressing the total requirement and expectations of the end user. This entails that the government, community and health facilities, must work together to ensure they address stigma and discrimination against PLHIV.

\subsubsection{Health facilities recognise that PLHIV loves interacting with their peers, and creates opportunities for such interaction as part of the continuum of care process}

It emerged from the FGD that participants expect health facilities to recognise that PLHIV loves interacting with their peers, and create opportunities for such interaction as part of the continuum of care process. PLHIV have preference for association and experience sharing with their peers who are also living with HIV. Support group structures could be useful platforms for ensuring quality continuum of AIDS care and drug adherence. Support group members could be better treatment supporters, care givers and volunteers in AIDS care than people who are not living with HIV. Support group of PLHIV could be a better platform for end user tracking and follow up. Additionally, it came to the fore from the study that support groups are emerging meeting points for development of long term/marital relationships among PLHIV. Regular activities at the support group forum range from encouraging participation in support group meetings, use of support group volunteers as adherence counsellors, HIV counselling and testing as lay counsellors, home based care volunteers, and so 
forth. Participants of this study opined that PLHIV prefer to be counselled by fellow HIV positive persons.

A participant expressed her view concerning the level of trust and freedom between PLHIV as quoted in her own words below:

\begin{abstract}
"Somebody who is positive will be more at home when handled by a HIV positive person. Somebody wey [that] meet somebody who is HIV positive and say, even me, I am positive myself. Start building... that same person will say are you positive? He'll say are you truly positive? And say, yes! Because I know, I am an adherence counsellor, so I know people who come in. I know how they feel when I tell them I am also positive. They come down well."
\end{abstract}

The PLHIV community forms an emerging community resource that makes up the element of the CCM, referred to as community resources. To enable this emerging community to function maximally, network and coordinate with the wider community for relevant services, the health facility needs to provide fundamental recognition to this community of PLHIV. The PLHIV support group community serves as a resource for services that cannot be provided by the health care workers, such as home based care, contact tracing and follow up, adherence counselling, among others. Wielawski (2006:6) related this element of $\mathrm{CCM}$ to encouraging end users to participate in community activities, and mobilising community resources to meet the needs of PLHIV.

Minkman et al (2007:91) assert that successful implementation of interventions based on the six elements of CCM may result in productive interactions between informed and activated end users and prepared and proactive care teams. Additionally, the successful coordination and implementation of interventions based on the six elements of the CCM and between the community and health facilities may result in better functional and clinical outcomes (Minkman et al 2007:91). HCIP (2008:1) identifies engaging teams of managers, service providers, and community stakeholders as a strategy for continuous quality improvement in ART care. According to Wagner et al (2007:871), lessons learned from Uganda, Zambia and South Africa show that to minimise end users dropping out of care, structures (such as support group for PLHIV) should be developed to enable end users provide support to each other, and have a "safety net" for helping each other remain in care. 
The perceptions of participants above is also corroborated by Torpey et al (2008:1) who report that ASWs who are mostly PLWHA may be in a better position to provide empathic and emotional support to end users as well as providing community follow up which remains a challenge among HCWs. Hasin et al (2001:1) elucidate that end users should not be left alone for a long time, which needs more nurses appointed in the health facility. Where nurses and other health workers are not available, effort should be made to fill with volunteers. The health facility's long-term survival would ultimately be determined by the extent to which the facility's end users are satisfied or dissatisfied. This notion would be ingrained in the mind of every existing and new health care provider and volunteers.

\section{Conclusion}

It emerged from the study as featured in this sub-theme that end users perceive health facilities to recognise that PLHIV loves interacting with their peers, and create opportunities for such interaction as part of the continuum of care process. The PLHIV community serves as a community resource for services that cannot be provided by the health care workers, such as home based care, contact tracing and follow up, adherence counselling, among others. This element of CCM, community resources, therefore encourages end users to participate in community activities, and mobilising community resources to meet the needs of PLHIV.

\subsubsection{Health facilities continuously improve quality and processes to meet the changing requirement and expectations of end users}

Analysis of FGD indicated that participants expect health facilities to continuously improve quality and processes to meet the changing requirements of end users. End users increasingly demand more sophisticated type of care; therefore, health facilities should improve internal processes to reduce end users' dissatisfaction with services. Health facilities should institute measures to prevent extortion of end users by syndicates. It was evident from the data that end users demand automation of record keeping systems to enhance easy storage and retrieval of data. Participants also demand segmentation of record retrieval terminals by age, such that adults and children are not seen to be collecting folders from the same terminal. Participants demand gainful employment of PLHIV. End users also demand support in form of micro-credit 
scheme for support group members. Additionally, end users demand uninterrupted, affordable or free supplies of basic care kits; in addition to assistance in form of food and food supplements.

End users' requirements, just like change is constant, is always dynamic. What an end user will request at one point may not be the same when asked again in the next hour or in the evening of same day. The function of health facilities practicing TQM within the context of CCM is therefore; to continuously gauge the changing needs of end users and adopt continuous quality improvement to meet the changing requirement and expectations.

CCM is a TQM tool used mainly to optimise care of a specific end user group (such as PLHIV attending ART) at the more operational or process level (Minkman et al 2007:102). It is therefore a convenient framework for addressing quality and process related issues. The function of the $\mathrm{CCM}$ in relation to this finding relates to the element called health care organisation. Wielawski (2006:6) and Singh and Ham (2006:5) elucidate that this element relates to creating a culture, organisation, and mechanisms that promote safe, high quality care, including making excellence a priority and pursuing it visibly.

It involves continuously reviewing processes (process flow) and redesigning the processes to yield maximum value, improved efficiency and effectiveness. This entails implementing continuous quality/process improvement in the health facility. CQI looks for opportunities to continually improve processes and to identify ways of doing things better. Therefore, the most successful quality improvement plans have been those recognising and defining the quality from the viewpoint of the end user (Mehrabi et al 2007:563). In health facilities, the effectiveness of quality improvement models is evaluated with regard to end user needs and experiences (e.g. the satisfaction of end users or their companions) (Mehrabi et al 2007:563).

Findings of a study in Sao Carlos Hospital School showed that the adoption of a humanised and user focused model of assistance in health care in this hospital resulted in end user satisfaction (Ricci et al 2011:1125). Booysen et al (2007:283) report a South African statistical analysis of cross-sectional data which reveals that it is not access to treatment per se that enhances the quality of life of those who have come forward for 
ART. Rather, it is the health benefits associated with treatment, levels of stigmatisation, quality health care services, and the ability of persons to access support and care, both from within and outside the health care facility, which contributes to end user satisfaction. UHCIP (2008:1) note that the focus on end user satisfaction is central to the quality improvement framework, which perceives the primary purpose of health services as being to meet the needs and improve the health and well-being of the clients who use them. The emphasis on systems and processes of care is also central, since poorly designed systems generate inefficiency and waste, poor health care quality, and negative health outcomes.

Francois et al (2003:52) report a teaching hospital's implementation in response to the principles and the theory of CQI, in the following ways: (i) it emphasises organisational processes as causes of weaknesses rather than blaming individuals; (ii) it uses structured problem-solving methods (iii) multidisciplinary work groups are set up (iv) staff members are given responsibility; and ( $v$ ) it addresses both internal and external clients (health facility employees and end users) explicitly.

\section{Conclusion}

Analysis of the study featured in this sub-theme indicated that end users perceive health facilities to continuously improve quality and processes to meet the changing requirement and expectations of end users. The function of the CCM in relation to this finding relates to the element called health care organisation. This element relates to creating a culture, organisation, and mechanism that promote safe, high quality ART care, including making excellence in ART service provision a priority and pursuing it visibly.

\subsection{THEME 3: ART SERVICES THAT DISAPPOINTED PARTICIPANTS FROM MEETING THEIR REQUIREMENTS (DISSATISFACTION)}

This theme relates to ART services that contributed in the dissatisfaction of end users attending public health facilities. Eight sub-themes appeared to relate to this theme. The following aspects of ART service provision failed to meet end users' requirement and expectations: weak responsiveness by health facility leadership, inadequate space and aesthetics, and frequent interruptions in ART laboratory services. Others include 
inadequate courtesy and mechanisms for managing complaints, sub-optimal standardisation of processes and procedures, inadequate measures for ensuring usersafety and interrupted supplies of drugs for opportunistic infections. Gaioso and Mishima (2007:1) note that dissatisfaction in their own study was predominated in terms of environment and organisational accessibility, impairing care longitudinality and continuity. Medeiros et al (2010:402) indicate poor health facility infrastructure (physical area) as a factor of dissatisfaction. Regmi and Madison (2010:44) report that dissatisfaction was expressed regarding some aspects of their study, viz, delivery of care, especially, the lack of privacy and confidentiality and the absence of a support person from the end user's own family.

These factors were highly regarded by the end users as necessary for their satisfaction with overall health care (Regmi \& Madison 2010:44). Wouters et al (2008:11) report that overall, dissatisfaction with waiting times seems to have been the most important predictor of discontent among end users receiving ART. Failure to engage with the end user's agenda can lead to misunderstandings, dissatisfaction, and poor outcomes (Coulter 2005:1200). Coulter (2010:4) reveals that not being properly informed about their illness and the options for treatment are the most common causes of end user dissatisfaction. Similarly, Nakhaee and Mirahmadizadeh (2005:192) espouse that the provision of insufficient information regarding other health care caused dissatisfactions among the end users.

This is a complex of multidimensional areas, which require an integrated model with features to addressing both internal systems and processes (including service integration), and 'horizontal' coordination, collaboration and partnership with other organisation (Minkman et al 2007:91). The CCM has features for recognising and addressing this complex - coordinating between the community, health system and the four other elements within it. Where the complex requires more techniques, the CCM also has an expanded model which has extras relating to end user safety, staff development, cultural aspects, coordination and other dimensions of quality (Minkman et al 2007:91).

Each of the eight sub-themes of this theme are discussed in more detail, below. 


\subsubsection{Overstretched, space constrained and aesthetically non-attractive ART care infrastructure}

Participants explained during the FGD that their ART service sites are overstretched; experiencing major space constraints and aesthetically non-attractive to sight. End users attending ART services in the health facilities are overcrowded beyond the space originally provided for ART services. Constrained storage space and inadequate lighting is also an issue in the health facilities. Similarly, there is inadequate number of seats to match end user population on clinic days. ART clinic environment is unattractive and non-condusive for end users, but participants from 1 of the 6 health facilities investigated said their health facility is clean and attractive. In general, while end user enrolment into the ART programme continues to increase day-by-day, no commensurate effort is made by Nigerian health authorities to expand the infrastructure. The constrained space and infrastructure therefore, serve as a source of dissatisfaction for heath service users.

This is a challenge with delivery system design, one of the six elements of CCM. Wielawski (2006:6) and Singh and Ham (2006:5) report that this element relates to delivering effective, timely and efficient care within an adequate infrastructure. Pietrovsk and Dall'Agnol (2006:630) indicate aesthetics and cleanliness of the health facility environment as factors that interfere with end user satisfaction. Gaioso and Mishima (2007:1) note that dissatisfaction in their own study was predominated in terms of environment and organisational accessibility, impairing care longitudinality and continuity. Medeiros et al (2010:402) indicate poor infrastructure (physical area) in BHU as a cause of dissatisfaction among end users. Similar finding was also reported in Kenya by Agha and Do (2009:87).

\section{Conclusion}

The study findings revealed as elucidated in this sub-theme that ART service sites are overstretched; experiencing major space constraints and are aesthetically non-attractive to sight. This finding evolves from failure in delivery system design, one of the six elements of CCM. This element relates to delivering effective, timely and efficient care within an adequate infrastructure. 


\subsubsection{Frequently interrupted laboratory services, related to high equipment down time and commodities stock-out}

This finding relates to the frequency of breakdown of laboratory equipment and laboratory commodities stock-out, resulting to interruptions in laboratory services provision. Equipment breakdown and laboratory reagent stockouts formed common causes of interruptions in laboratory services. Number of end users on waiting list for laboratory tests places excessive burden on the laboratory equipment, with eventual breakdown. Weakness in laboratory systems, including inadequate health care workers and sub-optimal capacity to plan effectively, interrupt laboratory services provision; and end users pay the price of poor quality caused by inadequate health systems. This situation has caused end users to return home without having tests conducted. Repetitive visits without tests create boredom and dissatisfaction among end users. At times, even when the machines are functional and lab reagents are available, end user overload undermines the lab staffers desire to do all the tests the same day. Professional and original equipment manufacturer's standards also provide limitations to the number of tests a lab staff can do per day. Against this background, they tend to select a few number of end users to be attended to that day, and ask others to come back another day. Some of the participants lamented their experiences with frequently interrupted laboratory services, thus:

\footnotetext{
"...there are sometimes we come here to do CD4 count. So many times, we come, no facilities to do the test. Still, they don't allow somebody to do the test outside. Sometimes, some people like I, came here more than 5 times ...for some of us, we don't always have time. When you come, I don't know the name, OK, reagent, there's no reagent. You have to continue coming. Sometimes when you want to collect your drugs from the pharmacy, they'll tell you without the CD4 they'll not give you drugs. So sometimes, we don't know what is happening like that..."
}

This is another factor of inadequate service delivery system design, causing interruptions in the ART service delivery process. Since the CCM promotes evidence based service delivery, and lab measures serve as bases for obtaining evidence in the ART care process, it behoves that it is a fundamental issue for program managers' attention. Wielawski (2006:6) and Singh and Ham (2006:5) report that this element, delivery system design, relates to delivering effective, timely and efficient laboratory services within an adequate infrastructure. The infrastructure here includes functional laboratory equipment with available reagents sufficient to carry out tests without 
interruptions. This finding is corroborated in Chiegil (2010:50) who reports a study conducted in Nigeria, that laboratory tests are not routinely done for end users despite frequent visits to the health facility. Alemayehu et al (2009:360) reports that a relatively better quality of laboratory services was provided to end users on enrolment into care, but failed to sustain the care during follow up care. Mfinanga et al (2008:3) report their study's findings which showed that about $15,0 \%$ to $34,8 \%$ of end users were not satisfied with waiting time and timely instructions (among others) during laboratory services. Another study from Ethiopia was reported by Alemayehu et al (2009:360) that a relatively better quality of care was provided to end users on enrolment in care, with 90,9\% of the end users receiving their first CD4 count within 2 weeks after first HIV clinic visit. However, the health facility failed to provide recommended care for significant portion of its HIV infected end users, during the follow up period.

\section{Conclusion}

In this sub-theme, data analysis revealed that participants are constantly frustrated with the frequently interrupted laboratory services, arising from frequent equipment breakdown and laboratory reagent stock-out. This finding relates to the CCM element, delivery system design, which refers to delivering effective, timely and efficient laboratory services within an adequate and acceptable infrastructure. The infrastructure includes functional laboratory equipment with available reagents sufficient to carry out tests without interruptions.

\subsubsection{Inadequate supply and availability of drugs for opportunistic infections and multivitamins}

It emerged from the FGD that participants felt end users experience inadequate supply and availability of drugs for opportunistic infections and multivitamins. Health facilities experience outages of OIs and sometimes, second line ARVs. Nutritional supplements are also not consistently provided in the health facility. When end users come to the health facility, they expect that they will be given drugs to address all complaints they made to the doctor. On getting to the pharmacy, they are sometimes told they could only receive free supplies of ARVs. They are either made to pay for some of the drugs for opportunistic infections (OI) and multivitamins or simply told these drugs are out of 
stock. Those who have money buy the drugs, while others keep taking ARVs without medications for OI management.

Participants complained about the non-availability of Ol drugs and multivitamins. A direct quote from a participant concerning her challenges with availability of $\mathrm{Ol}$ drugs and multivitamins are indicated thus:

\footnotetext{
“...we get our ARVs each time we come for it. We only encounter challenges with drugs for Ols, which are usually out of stock. Sometimes we also experience stock out of second line ARVs."
}

CCM refers to inadequate commodity security as a flaw in delivery system design. According to Wielawski (2006:6) and Singh and Ham (2006:5) this element of the CCM, delivery system design, could address logistics systems and put a system in place that addresses effective, timely and efficient supplies of OI drugs and multivitamins. Other study reports from reviewed literature highlights similar findings in Ethiopia and other sites in Nigeria. Karunamoorthi et al (2009:331) sought to determine end users' level of satisfaction and their expectations with pharmacy services at specialist ART unit in the government health facilities of Addis Abbaba, Ethiopia. Results of the study showed that end users faced inadequate supplies of drugs, but could not tell what the cause of drug outages were (Karunamoorthi et al 2009:331).

Alemayehu et al (2009:360) report a study in Ethiopia, where out of the sample end users living with HIVIAIDS who were eligible for cotrimoxazole (CPT), only about 45,9\% were actually on CPT. This considerably low achievement was because of failure to prescribe CPT for eligible end users during clinic visits, lower rate of continuity of care and poor compliance of end users with CPT. The study report was silent on whether or not there was stock-out of CPT and other Ol drugs. A similar finding of frequent stockout of Ol drugs and multivitamins in the Nigeria ART programme was noted by Chiegil (2010:50). This could form part of the major potentials for end users' dissatisfaction regarding provision of ART in Nigeria.

\section{Conclusion}

It emerged from the study as argued in this sub-theme that end users experience inadequate supply and availability of drugs for opportunistic infections and 
multivitamins. A review of this finding against CCM standards reveals a possibility of weakness in delivery system design. This element of the CCM, delivery system design, could address logistics systems and put a system in place that addresses effective, timely and efficient supplies of OI drugs and multivitamins.

\subsubsection{Perceived increasing incidences of failure to treat end users with courtesy, related to inadequate health care workers and staff burnout of the available few}

Participants explained during the FGD that the health facilities increasingly failed to treat end users with courtesy. End users face experiences of discrimination, intimidation and being scolded by health care workers. There is dearth of skilled and experienced health care workers to provide quality ART services. Health care workers are inconsiderate in meeting the demands of end users. There is increasing health care worker burnout as a result of excessive end user load. Despite popular opinion among participants on dissatisfaction with courtesy of health care workers, very few end users confirmed that they were treated with passion. Overall, participants elucidated their personal experiences with a few health facility staff that made them felt discouraged of coming back to the health facility. Limitations in health care workers might have contributed to placing excessive burden on facility staff, due to high end user load. It is likely that a burntout staff will become aggressive and transfer such aggression to end users through treatment without courtesy.

A participant recounted his experience with the courtesy of nurses in the ART clinic, which he described as very bad. He said thus:

\footnotetext{
"...the nurses they call matron or something like that, the way they shout on people having this virus. It is as if there's no, or you are no more a living person. They will see you now [and say]... "please, see me, see me, na me give you sickness?" They are not even telling us that there will still be life. That is why I always ask ...may be they don't know how someone in this problem feels... In fact, they are very harsh. ... may be the person's mouth may be having odor or your ear sef may be having problem, not able to hearing something clear. Something like that. They'll be shouting at you, that's my own experience, these are not even exaggeration or saying they say. That is how they did it to me and up till now, that's how they are treating patients, especially, new patients there. My own is easier... the way they handle them [new end users] is very, very bad, very poor for their services."
}

This finding relates to self-management support, which is an element of the CCM. The element relates to empowering and preparing end users to manage their health and 
health care. It also looks at setting a tone of collaboration and professional intimacy between health care workers and end users, to encourage the latter's participation in setting personal goals, adapting self-care and fine-tuning treatment (Wielawski 2006:6 and Singh and Ham 2006:5). Chow et al (2009:438) affirm that affability or interpersonal manner of health care worker is often thought of as the principle component of end user satisfaction. Udoh et al (2011:221) hypothesise that the mode of health care delivery appears to be more important to end users than the actual care itself. This hypothesis agrees with Coulter (2005:1199) who infers that end users want health care workers who are good communicators and have sound, up to date clinical knowledge and skills.

They also want health care workers who are interested and sympathetic, involve them in decisions, give them sufficient time and attention, and provide advice on health promotion and self care (Coulter 2005:1199). Yakong et al (2010:2431) described major themes that emerged from a study sample of women attending health care, which include experiences of intimidation and being scolded; experiences of limited choices; receiving silent treatment, and experiences of lack of privacy. In another study in Brazil in 2003, Gouveia et al (2005:S109) espoused that multiple regression analysis showed that having experienced some type of discrimination (on the basis of gender, age, poverty, social class, skin colour, or type of disease) and being an exclusive user of the public National Health System involved a lower degree of users' satisfaction. Findings from a US based study by Ding et al (2008:40) indicate that about 96\% of end users in their sample (representing about 217,000 end users nationally) said that a physician, a nurse practitioner or a physician's assistant knew them the best and took responsibility for their overall HIV medical care.

Only about $4 \%$ of the end users did not identify a primary HIV care provider.Another US based study found that end users who report greater involvement in medical care are more satisfied with their health care workers (Beach et al 2007:1119). They also report more understanding, reassurance, and perceived control over their illness, and have improvements in medical conditions. Another study found that end users who prefer a more active role are less satisfied when their physicians do not support their preference (Beach et al 2007:1119).

In contrast to the US based study, and with similarities to the current study, a study finding from South Africa showed that, because human resource shortages are 
associated with lower levels of end user satisfaction, the quality of care is definitely affected by heavy workloads (Wouters et al 2008:11). Wouters et al (2008:11) add that human resource shortages, combined with large numbers of end users in need of treatment, caused further extended waiting times, which were the most important source of discontent among the PLHIV. This could seriously hinder any successful ART scaleup, because several previous studies have shown that both waiting times and low end user satisfaction levels with ART-related services hinder ART adherence (Wouters et al 2008:11).

This finding was corroborated by Hasin et al (2001:11) who opined that poor courtesy from health care providers may be associated to inadequate information (specifically on price and services), carelessness of health care providers and shortage of officers, among others. The labour-intensive nature of ART delivery set against health workforce shortages in developing countries has made human capital an issue of particular focus within the decade (Tobi, George, Schmidt \& Renton 2008:1452). Adding that with more funding available for ART, capacities of health systems and shortages of workers have replaced funding shortfalls as the most serious threats to achieving universal ART coverage.

Three elements can help end user feel well treated: face-to-face communication; skilful providers who show end users that they care about their work; and consideration of how women's needs, fears, and reactions may be perceived differently by male and female providers (Creel et al 2002:4). Some projections made at conceptual level, Tobi et al (2008:1452) report that ART scale-up can lead to a diversion of attention, resources and personnel from other essential health services, such as focusing on quality of care.

The AHRQ (2010:153) elucidates that end user centeredness encompasses qualities of compassion, empathy, and responsiveness to the needs, values, and expressed preferences of the individual end user. End user-centred care is supported by good provider-end user communication so that end users' needs and wants are understood and addressed and end users understand and participate in their own care. This approach to care has been shown to improve end users' health and health care. In contrast to the findings above where end users verbalised dissatisfaction with care, AHRQ (2010:156) notes that from 2002 to 2007, the percentage of adults with a doctor's office or clinic visit in the US, who reported poor communication significantly 
decreased, from $10.8 \%$ to $9.3 \%$. In all years, adults with basic or complex activity limitations were more likely to report poor communication than adults with neither basic nor complex activity limitations. It is therefore, important that both end users and providers not assume that instructions are understood but develop a means to show comprehension.

\section{Conclusion}

It emanated from this study as discussed in this sub-theme that end users perceived increasing incidences of failure to treat them with courtesy. Some of them thought this experience could be related to inadequate health care workers and staff burnout of the available few. This finding relates to self-management support, which is an element of the CCM. The element looks at setting a tone of collaboration and professional intimacy between health care workers and end users, to encourage the latter's participation in setting personal goals, adapting self-care and fine-tuning treatment.

\subsubsection{Weak responsiveness to the needs of end users by the health facility leadership, policy and strategy}

It emanated from the FGD that participants felt the health facility leadership, policy and strategies are weak in responding to the needs of end users. Public health facilities may have the desire to support end users, but have insufficient authorities to do so beyond their limits. Participants perceive that changes in health facility leadership might have contributed to weak responsiveness to their demands. Some health care workers demonstrate interest to be responsive to end user demands, but lack necessary authority to go beyond their authorised limits.

Leadership support for support group activities is encouraging in a few health facilities studied. Overall, while a few health facility leaderships recognise and work closely with PLHIV support groups to respond to their needs, others do not. Since TQM requires that what needs to be done should be done right first time, and be done right throughout all sections of the health facility, failure to meet these criteria supposes that the health facility leadership is not responsive to the needs of end users. 
The element of CCM that looks at leadership and policy issues as relevant to this finding is the decision support. This element relates to promoting health facility management and the overall care process, consistent with evidence and end user preferences (Wielawski 2006:6; Singh \& Ham 2006:5). Feedback from end users should form sufficient evidence for health facility leadership to take action.

In Zambia, Schumaker and Bond (2008:2130) report that end users often move from clinic to clinic seeking better treatment, aware of drug shortages that means they may not be prescribed the most appropriate or effective drug. In other cases, overstretched health workers may not explain a drug's action in the body, leaving end users to rely on past experience of pharmaceuticals and their own traditional and biomedical knowledge to interprete side effects and efficacy after they begin treatment (Schumaker \& Bond 2008:2130). The AHRQ (2010:153) elucidates that health facility leadership should encompass qualities of compassion, empathy, and responsiveness to the needs, values, and expressed preferences of the end users. End user-centred care is supported by good provider-end user communication so that end users' needs and wants are understood and addressed and end users understand and participate in their own care. Failure to engage with the end user's agenda can lead to misunderstandings, dissatisfaction, and poor health outcomes (Coulter 2005:1200).

\section{Conclusion}

It emanated from the study as elucidated in this sub-theme that the health facility leadership, policy and strategies are weak in responding to the needs of end users. This finding relates to the decision support element of CCM which uses evidence to influence leadership and policy issues as relevant to ART service provision. This element relates to promoting health facility management and the overall care process, consistent with evidence and end user preferences.

\subsubsection{Perceived increasing trends of inadequacy in ensuring end users emerge from the health facility healthy}

Analysis of FGD showed that participants were of the opinion that end users perceive increasing trends of inadequacy in ensuring that they emerge from the health facility healthy. Participants perceive that quality ART service provision is increasingly 
deteriorating with increasing number of new enrollments. Health facilities across the country are not at the same level of user focused quality. Overall, participants elucidate their experiences, where according to them; the quality of service seems to be dwindling downward, over time. As the number of end users increases on the programme, end users notice health care providers' withdrawal from paying greater attention to detail. Health assessments to determine their state of health and establish a baseline for evidence based care are apparently no longer available. Health care providers only try to take their body weight during clinic appointments, and fail to take blood pressure and other vital signs. When confronted, health care providers lay blames on excessive workload. Clinic visits are now synonymous to ARV refill visits, since end users only come to pick up drugs, with inadequate monitoring of their body systems.

A participant recounts her experience with the inadequacy in ensuring end users are properly assessed and results used to improve quality ART service provision. She lamented thus:

“...they don't do us BP [blood pressure], because they say we are too many. Before they do us everything. They start from vital signs, then weight, they do you BP, then, before you'll start seeing doctor. Since this new people [leadership] came ...there's nothing like that. They only do us weight. Then I requested and said, this thing is very important because somebody's BP may rise he'll not know, how will he know? They said that we are many, so they'll not be doing it."

Health measurements are important factors for generating evidence-based data for improving quality of care. In CCM, this element is called clinical information systems. The element relates to organising end users and population data to facilitate efficient and effective care (Wielawski 2006:6; Singh \& Ham 2006:5). It also facilitates population studies, for example, to measure health care performance against quality benchmarks, over time. Failure to measure vital signs and other body indicators is itself, a mark of poor quality ART service provision.

To maximise impact on treatment outcome, it is important for ART programmes to develop effective mechanisms for coordinating care to ensure that end users emerge from the health facility better than the way they came (Wagner et al 2007:871). Lessons learned from selected clinics in Uganda, Zambia and South Africa indicate that effective organisational management and human resource policies are essential to maintain high job performance, satisfaction and limit burnout, to ensure that end users get the best 
care possible and go back home as better people (Wagner et al 2007:871). To achieve this, Hopkins et al (2009:927) lament that end users expect their care to include building interpersonal relationships, one-on-one counselling, self-help groups, educational sessions, and information opportunities, communication with knowled-geable and empathetic staff and discharge planning. Hasin et al (2001:10) note that there are many weak operations in the health system that should be addressed properly. However, health care providers need to be more careful to reduce mistakes, utilise time and other resources. The health care providers should examine end users appropriately, provide required medication and correct health information when end users are leaving the clinic or health facility. To augment communication efficiency, the health care providers should also double check and ensure that the end users understand the communication in the same way they intended to pass on to them (Hasin et al 2001:1).

\section{Conclusion}

Findings of the study as discussed in this sub-theme showed that end users perceive increasing trends of inadequacy in ensuring that they emerge from the health facility healthy. Health measurements are important factors for generating evidence-based data for improving quality of care. In CCM, this element is called clinical information systems. This element relates to organising end users' data to facilitate efficient and effective care.

\subsubsection{Inadequate processes and procedures for managing end users' complaints}

It emerged from the FGD that when participants experience challenges in the course of seeking quality care in the health facility; they lack guidance on who to contact to lay their complaints or grievances. End users have complaints to raise against health care workers, but are unaware of the procedures for doing so, or processes for complaints management. The health facilities have not shared their complaints management procedures and processes; therefore they are either non-existent or inadequate. The absence or lack of clarity of complaints management procedures make end users to linger around with their complaints, which rather builds up to cause frustration, nonadherence to ART, loss to follow up and possible failure to patronise the facility for continuing treatment. If not properly managed, this situation may escalate into a crisis 
situation between health care authorities and end users. This could be as mild as spending more on unnecessary waste and rework. It could also be as serious as law suits and malpractice claims or end user protests for revolution.

Wielawski (2006:6) asserts that the CCM emphasise the need for health facilities to stay on top of clinical responsibilities while helping end users become active participants in their care. Ding et al (2008:40) elaborate that care can be poor even at sites with good support services if there is not a suitable health care worker relationship. The effective use of medical and support services probably requires a health care worker who knows each end user and can facilitate the provision and integration of care (Ding et al 2008:40). Madeiros et al (2010:402) affirm that a relationship involving respect and health care workers really listening to their end users makes the difference in health provision. However, the complaints made must also be considered for effective embracement of the service being provided in the health facility (Madeiros et al 2010:402). Wouters et al (2008:210) report from a study in South Africa that because human resource shortages are associated with lower levels of end user satisfaction, the quality of care is definitely affected by heavy workloads. Human resource shortages, combined with large numbers of end users in need of treatment, caused inadequate relationship building and further extended waiting times. These in turn were the most important sources of discontent among PLHIV attending the ART assessment sites studied in the Free State.

Gallaitry et al (2005:367) report that one of the most widespread complaints from end users, in general, is that of not receiving sufficient information about their illness and treatment, or the information that is provided is often too complex to understand and retain. Managing complaints requires active participation and coordination between the community and health facility. The component of the CCM that address the linkage between health facility and the community based opportunities is called community resources. This element relates to mobilising community resources to meet the needs of end users with long-term conditions such as PLHIV (Wielawski 2006:6; Singh \& Ham 2006:5). The CCM requires that health facilities identify and establish systems and processes for ensuring adequate relationship between the health facility and communities, and encourage end users to participate. The system also inculcates role clarification and preventive complaints management through open, clear and transparent communication. 


\section{Conclusion}

As discussed in this sub-theme, it emerged from the study that when end users experience challenges in the course of seeking quality ART services in the health facility; they lack guidance on who to contact to lay their complaints or grievances. This indicates that there is inadequate complaints management mechanism in place. Managing complaints requires active participation and coordination between the community and health facility. The component of the CCM that relates to this is community resources. This element relates to mobilising community resources to meet the needs and expectations of end users with long-term conditions such as PLHIV.

\subsubsection{Sub-optimal standardisation of health care processes and procedures, with resultant accumulation of bottlenecks along the workflow}

Analysis of FGD revealed that participants were of the view that health facilities implement sub-optimal standardisation of health care processes and procedures. This is evidenced by the accumulation of bottlenecks along the workflow, point of service or workstations. End users come out as early as 5 in the morning and spend long hours in the health facility, waiting to access ART service. Identified process improvement gaps are left unresolved, due to inadequate capacity and shortage of skilled human resources. Support group leadership serve as end user advocates and intervene at critical times to facilitate access to treatment for their peers.

End users brainstorm and come up with innovative ideas on improving processes, removing bottlenecks and reducing long waiting time. Syndicates use health facility's inadequate processes and procedures as opportunity to estort money from end users; while end users continue to bear the cost of poor quality caused by health care workers. Overall, once processes are continuously improved, and performance standardised across all workstations (removing departmental barriers), outcomes will come out faster and hitch free. The newly and improved performance or operations and processes will hopefully meet end users' requirement and expectations.

End users seemingly bear the cost of process flaws that were not caused by them, or that they knew nothing about. For example, when doctors fail to adequately fill out 
laboratory request forms, processed lab results fail to find their way into the appropriate user-folders. Thus, when end users come to be reviewed with lab results, they are told the results cannot be traced. In some cases, they will have to repeat the test and return on another day to be reviewed with the new lab results. This way, they incur extra out of pocket costs due to the health facility's operational lapses. A participant lamented his frustrations with the system, thus:

“...the CD4 count, they said the doctors didn't write the file number on the file. That one they won't put it in the files of the patients. When the patients do the CD4 count, to see the doctor now, you won't see the CD4 count result. So, the people that looking for the file, dey incharge of that thing. I went to them and said that how manage, about 10 people, including me that very day [have no results in the file]. I went there and said what is the reason? He said because the doctor didn't write the file number on the form. I said Ok, I started going to doctors round that they should write that number so that it should be there. So, when this thing getting too much, I tell...the person who in charge of that, that give me that file that you are putting CD4 count, the one that is not in the file, because it full file. I said let me help you call the names so that before they start seeing doctors. They refused, saying is not our job."

The CCM element that addresses organisational wide or management related processes and procedure is the health care organisation. This element relates to creating a culture, organisation, and mechanism that promote safe, high quality care; makes excellence a priority and pursues it visibly (Wielawski 2006:6; Singh \& Ham 2006:5). Essential to achieving this is the health care workers' participation and open communication with end users on errors and failings, as well as processes and strategies for coherent system improvement and spread (Wielawski 2006:6; Singh \& Ham 2006:5). According to AHRQ (2010:147), timeliness refers to the health care system's capacity to provide care quickly after a need is recognised.

Findings reported by Wouters et al (2008:11) showed that, because human resource shortages are associated with lower levels of end user satisfaction, the quality of care is definitely affected by heavy workloads. Wouters et al (2008:11) add that human resource shortages, combined with large numbers of end users in need of treatment, caused further extended waiting times, which were the most important source of discontent among the PLHIV. This could seriously hinder any successful ART scale up, because several previous studies have shown that both waiting times and low end user satisfaction levels with ART-related services hinder ART adherence (Wouters et al 2008:11). 
Process improvements have proven helpful in reducing waiting time in health facilities. For an example, Wong and Bradley (2009:253) sought to evaluate the impact of an inexpensive business process re-engineering project on the accessibility and completeness of end user information and satisfaction in Ethiopia. Findings reveal that medical record accessibility and completeness, and satisfaction improved significantly $(p<0.05)$ based on pre- and post-intervention comparisons.

Thus, depicting that a well-organised medical record management system can be effective in reducing waiting time and improving end user information accessibility and completeness in health facilities in low-income countries, despite the lack of resources (Wong \& Bradley 2009:253). Lessons learned from projects in Uganda, Zambia, and South Africa also provide that processes can be improved, and end user waiting time reduced. For example, Wagner et al (2007:871) reveal that minimising bottlenecks to smooth end user flow requires efficient staff allocation to appropriate clinical duties, streamlining clinic visit schedule protocols, and tapping end users and the HIV community as a key source of labour.

Limited human resources coupled with stressful working conditions/environments can result in overwork and burnout, and finally poor quality of care (Tobi et al 2008:1452). Others argue that ART scale-up is an opportunity to build strong and effective health systems, and in particular, the human resource capacity to provide quality care in the public sector (Tobi et al 2008:1453). Yet, evidence from countries around the world suggests that the health care provided for much of the world's population is of very poor quality and does not meet evidence-based standards (HCIP 2008:1). Studies show that providers routinely comply with only a small proportion of guidelines, even after standards-based training (HCIP 2008:1). Guidelines and processes are interrelated and depend on a series of cross-functional interventions.

It is the notion of work interrelatedness which drives the need for cross-functional teams. It calls for the breaking down of departmental barriers in the health facility. The basic internal processes necessary for this paradigm shift within the health facility includes: (i) changing the facility's structure in order to better identify and improve processes; (ii) using a quality oriented information system to study processes; and (iii) empowering employees and/or creating cross-functional teams to take charge of their 
operations in a manner that encourages continuous learning as well as personal responsibility (Hug 2006:287).

\section{Conclusion}

Findings of the study as espoused in this sub-theme revealed that health facilities implement sub-optimal standardisation of health care processes and procedures. The CCM element that addresses organisation wide or management related processes and procedure is the health care organisation. This element relates to creating a culture, organisation, and mechanism that promote safe, high quality ART care; and makes excellence in ART service provision a priority, and pursues it visibly.

\subsection{PARTICIPANTS' SUGGESTIONS FOR IMPROVING END USERS' REQUIREMENT FOR QUALITY ANTIRETROVIRAL THERAPY SERVICE PROVISION}

Participants' suggestions for improving end users' requirement and expectations for quality ART service provision was one of the major themes that emerged during data analysis. Four sub-themes appeared to relate to this theme. They include the following: health care workers training and re-training, service integration, commodities logistics and supply chain management and strategies to reduce end user overload in the clinic. Antiretroviral therapy service provision requires high end user involvement in the consumption process, and the traditional health care view of technical quality is inadequate to manage this complex (Gill \& White 2009:14). More importantly, Gill and White (2009:14) argue that effective health care relies on the co-contribution of and attention given to the end user in the service delivery process.

Each sub-theme is discussed in more detail, below: 


\subsubsection{Deploy, train and re-train additional health care workers to meet increasing number and dynamic expectations of end users in ART sites}

It emanated from the FGD that participants suggested that appropriate health authorities should deploy, train and re-train additional health care workers to meet the increasing number and dynamic expectations of end users attending ART. The deployment of health care workers especially doctors was pointed out during the study. The need for health care workers was highlighted to address the needs of the community. Training of health workers on sharing of information was also highlighted.

Training for all, as well as analysing the need for training and conducting training for all was perceived as a possibility for improving quality of ART service provision. Overall, the participants noted that the workforce planned over a decade ago when health facility patronage was low is still maintained now that all health facilities have exceedingly doubled their patronage. This raises the need to increase deployment or re-deployment of additional doctors, nurses, lab scientists, pharmacists and other professionals relevant to the care and treatment of HIVIAIDS. Such staff when deployed should be trained and/or retrained in the care and treatment of HIVIAIDS, to match current and emerging innovations in the diagnosis, care and treatment of HIVIAIDS.

The dynamics of poor service delivery often involve wasted effort, repetition and misuse of skilled employees (Gill \& White (2009:14). Essential to achieving health care quality is the health care workers' participation and open communication with end users on errors and failings, as well as processes and strategies for coherent system improvement and spread (Wielawski 2006:6; Singh \& Ham 2006:5). Limited human resources coupled with stressful working conditions can result in overwork and burnout, and finally poor quality of care (Tobi et al 2008:1452).

Therefore, building human resource capacity to provide quality care in the public health facilities is a formidable recommendation (Tobi et al 2008:1453). Hasin et al (2001:12) raises the need for developing schemes for staff development to meet the quality of care required in ART services. Adding that employees must be empowered; employee empowerment should not be misinterpreted as an excuse to abdicate leadership. Hasin et al (2001:1) also noted the need for job and process training for doctors and nurses, as well as for other officers and technicians. 


\section{Conclusion}

It emanated from the study as elucidated in this sub-theme that appropriate health authorities should deploy, train and re-train additional health care workers to meet the increasing number and dynamic expectations of end users attending ART.

\subsubsection{Integrate HIVIAIDS into regular health services; end users encouraged to attend regular clinics}

Analysis of the FGD revealed that participants were of the view that HIVIAIDS services should be integrated into regular health services, and end users encouraged on attending the regular clinics. Service integration reduces stigma and re-inforce end user rapport with their health care workers, especially, doctors. End users of ART services should attend regular clinics, throughout the week. These recommendations were given against the background of programme sustainability, reduction of stigma and discrimination and reduction of excessive end user load on the designated ART clinics. It was their opinion that running parallel clinics in the same health facility places excessive burden on the already overstretched human resources and health infrastructure.

Minkman et al (2007:91) report that end user-centered care focuses on the total needs of end users, not only on the services provided by one professional, clinic or organisation. Thus, sustaining seamless integrated care during the whole care process is most reasonable to achieving end user satisfaction (Minkman et al 2007:91). For the public health facilities, this requires horisontal coordination, collaboration with other organisations and community partners or service integration. The effective use of medical and support services probably requires a health care worker who knows each end user and can facilitate the provision and integration of care (Ding et al 2008:40). Jackson (2001:162) asserts that a better approach to implementing TQM is to explore creative ways to integrate the tool into current activities thereby applying the principles of it more effectively.

Francois et al (2003:48) share that their TQM intervention in a teaching hospital in France involved introducing quality management activities into each of the health facility 
departments participating in the study by setting up a specific organisation - the department's quality system. Providing integrated high-quality care also makes sense for service providers, since improving basic standards of care under an integrated platform attracts more end users, reduce per capita costs of services and ensures sustainability (Creel et al 2002:1). For example, the Bangladesh Women's Health Coalition attracts end users by providing a mix of services, so that end users can use a visit for more than one purpose, and by having well-trained paramedical personnel, rather than physicians, perform pelvic exams, intrauterine device (IUD) insertions, and menstrual regulation services. The high volume of end users has enabled the programme to distribute its fixed costs over a larger number of end users, allowing the coalition to serve more people at a lower cost (Creel et al 2002:1).

\section{Conclusion}

The finding of the study as discussed in this sub-theme revealed that HIVIAIDS services should be integrated into regular health services, and end users encouraged on attending the regular clinics.

\subsubsection{Improve supply chain management of laboratory reagents and drugs for opportunistic infections, to ensure availability and use}

Participants suggested during the FGD the improvement of supply chain management of laboratory reagents and drugs for opportunistic infections, to ensure availability and use. The cost of OI drugs limits end users' access to OI drugs, therefore, this should be addressed. Resolve laboratory reagent stockout by addressing logistics challenges centrally, at the national level. Overall, participants recommended that programme planners should make adequate quantification of commodities and commit resources to meeting end users' requirements for uninterrupted ART services.

This finding relates to strengthening logistics and ARV delivery systems to ensure uninterrupted and efficient supplies at all times (Wielawski 2006:6; Singh \& Ham 2006:5). Similar recommendations were noted in Chiegil (2010:51), to make drugs routinely available and accessible to end users, and make lab tests routinely available and accessible to end users. In North West Ethiopia, Alemayehu et al (2009:360) report that out of the sample end users living with HIVIAIDS who were eligible for CPT, only 
about 45,9\% were actually on CPT. This considerably low achievement was not related to availability, but failure to prescribe CPT for eligible end users during clinic visits, lower rate of continuity of care and poor compliance of end users with CPT.

\section{Conclusion}

It emanated from the study as highlighted in this sub-theme that strengthening supply chain management of laboratory reagents and drugs for opportunistic infections will improve availability and use.

\subsubsection{Reduce end user load in the clinic by reducing frequency of health facility visits by stable end users, through dispensing ARVs that will last 3 months per visit}

It emerged from the FGD that participants suggested that to reduce end user load in the ART clinics, health care authorities and service providers should consider reducing frequency of health facility visits by stable end users. Participants suggested that health facilities should dispense drugs that will last three months, during end user refill visits. This recommendation is a deviation from the current national ART guidelines which allows for dispensing only two months stock at a time. Some of the end users they noted, are already experienced in taking antiretroviral drugs, and do not need to be coming to the health facility every two months as currently programmed. The current scheme seems to be placing unnecessary strain on both the end user and the health facility. Chiegil (2010:51) recommends applying strategies necessary to reducing end user delays and waiting time in ART centres.

The suggestion made by the participants of this study could be considered for examination, to reducing current health facility overload. Gutierreze et al (2009:61) reveal that at the central pharmacy in Lima, waiting time was significantly higher in the sub billing process and also the time that users took on long queues was higher than the actual time. In Addis Ababa, Karunamoorthi et al (2009:337) show that end users were dissatisfied with the pharmacy services. Major area of dissatisfaction was long waiting time in the pharmacy, which gulped $82.5 \%$ of respondents investigated. In some cases, end users faced inadequate supplies of drugs, but could not tell what the cause of drug outages were (Karunamoorthi et al (2009:337). 
Participants recommend that the authorities concerned with updating the national ART guidelines should consider increasing the interval for ART refills to three monthly, up from the current two monthly refill period. This, they opine will significantly reduce end user load in the health facility and provide time for delivery of user focused care.

\section{Conclusion}

It emerged from the study as discussed in this sub-theme that health care authorities and service providers should consider reducing frequency of health facility visits by stable end users, in order to reduce end user overload in the ART clinics.

\subsection{CONCLUSION}

This chapter addressed the discussion of findings obtained from focus group discussions. The discussions of findings were guided by the themes and sub-themes that emerged from the analysis of participants' responses. Four major themes were discussed and aligned to the components of the CCM, as follows:

The first theme discussed in this chapter was ART services that met participants' requirements. Nine sub-themes were related to and discussed under this theme. Thus, it sprang from this study that uninterrupted supply of ARV drugs in public health facilities met end users' requirements for quality ART service provision. This finding was related to the 'delivery systems design' of the CCM, which promotes strengthening systems for delivering effective and uninterrupted ARV drug supplies to all service sites. It also emanated that some doctors, nurses and other health workers treated end users with courtesy; and their action served as source of encouragement for PLHIV. This finding was related to self-management support of the CCM. This element promotes empowering and preparing end users to manage their health and health care.

The study finding also featured that empathising with the end user while counselling improves quality of life of end users. This finding relates to self-management support element of the CCM. This element assumes that when health care workers empathise with end users, they instil confidence and ability 'to do it yourself' into the end users. It also stemmed from this study that ART knowledge explosion improves cohesion among 
PLHIV and raises demand for quality care. Increasing knowledge of ART among end users entails empowering and preparing them for taking more responsibility regarding their health. Therefore, this study aligned the finding to the self-management support element of the CCM.

Furthermore, it emerged from the study that support group members express enthusiasm to serve as volunteers to improve the quality of life of their members. Evidence generated from the work of the support group volunteers will contribute to decision-making regarding the need to recognise and institutionalise the role of support group in the health facility. In this study therefore, this finding aligns to the decision support element of the CCM. It further emanated from the study that support group volunteers promote infection prevention and control through triage of vulnerable members in the clinic. For the purpose of this study, this finding aligns to the CCM element, decision support, and relates to promoting care that is consistent with research evidence and end user preferences.

It also emerged from the finding that volunteer pharmacists improve the quality of pharmacy services in ART clinics. In this study, this element refers to clinical information systems, which relates to organising end users data to facilitate efficient and effective care, such as, organising the care team to measure the performance of pharmacy services against best practices or quality benchmarks. The finding of the study further revealed that strengthening linkages between support groups and community based organisations provide opportunities for wrap around services and improves the quality of life of PLHIV. In the CCM, the element that supports this finding is referred to as community resources. This element relates to identifying and mobilising community resources to meet the needs and expectations of end users with long-term conditions such as HIVIAIDS.

Lastly on this first theme, it emanated from the study that the leadership of support groups develop and implement innovative approaches aimed at improving dialogue with health authorities. The dialogue was aimed at improving relationships with health authorities and to address the requirement and expectations of their members. In CCM, this finding was seen to relate to health care organisation or health system. This element relates to creating a culture, organisation, and mechanism that promote safe, 
high quality ART service, including making excellence in ART service provision a priority and pursuing it visibly.

The second theme discussed in this chapter was the participants' perceived successes in ART service provision. Nine sub-themes were related to and discussed under this theme. Thus, it emerged from the study that end users expect health facilities to be spacious, clean and attractive, with adequate infrastructure that meets environmental health standards and the aesthetic needs of end users. This finding relates to the CCM element called delivery system design. This element relates to delivering effective, coordinated, uninterrupted and efficient care in an environment that is designed and accredited to meet acceptable standards of ART care. The study also featured that end users expect lifesaving ART programme to be fundamental to the survival of PLHIV, therefore, will not be allowed to experience service interruptions or complete seizure. The CCM element that relates to meeting the expectations of end users for ensuring no interruptions in ARV drug supplies is the delivery system design. This element relates to strengthening ARV logistics and supply chain management to ensure uninterrupted and efficient ARV supplies at all times.

It further emanated from the study that participants perceive that any end user who turns up to the health facility benefit from full assessment of his/her current health status; and is counselled on emerging clinical manifestations that need medical attention. In CCM this finding may require reviewing and re-aligning workstations and/or end user appointments in order to cope with the increases in their number, while also meeting their expectations for quality ART service provision (delivery system design). This element therefore, relates to strengthening internal systems for ensuring that all end users are routinely provided with basic assessments whenever they turn up for care in the health facility. Analysis of the study showed that end users expect health facilities to recognise the essence of PLHIV as volunteers, and design capacity building and motivational programmes to meet both the task requirements and volunteers' expectations. Activities related to this finding are described as self-management support, as an element of the CMM. This element relates to empowering and preparing end users to manage their health and health care.

Furthermore, the study finding featured that end users perceive health facilities providing ART services to have developed linkages with relevant facilities, to obtain 
specialised diagnostic services, and accept results arising from such facilities. Decision support element of the CCM requires that to link treatment with evidence, health care workers must have explicit guidelines on how to access specialised diagnostic services from other places, address laboratory results coming from outside their immediate facility or refer end users to facilities that have better capacity than theirs. It also emerged from this study that end users perceive health facilities to have standardised processes that ensure end users spend the least amount of time seeking care in the health facility. A system for time measurement or time series analysis in CCM is referred to as clinical information systems, which is one of the elements of CCM. This element relates to organising a management information system that helps to achieve timely, efficient and effective ART care.

Analysis of the study espoused that end users perceive government, health facilities, communities and individuals to have ensured a stigma free environment for PLHIV. The community is the base upon which the health system and four other elements of the CCM are housed. Therefore, this finding relates to community resources element of the CCM. The CCM has a characteristic feature of removing organisational boundaries in addressing the total requirement and expectations of the end user. This entails that the government, community and health facilities, must work together to ensure they address stigma and discrimination against PLHIV. It also emerged from the study that end users perceive health facilities to recognise that PLHIV loves interacting with their peers, and create opportunities for such interaction as part of the continuum of care process. The PLHIV community serves as a community resource for services that cannot be provided by the health care workers, such as home based care, contact tracking and follow up, adherence counselling, among others. This element of CCM, community resources, therefore encourages end users to participate in community activities, and mobilising community resources to meet the needs of PLHIV.

Finally on the second theme, analysis of the study featured that end users perceive health facilities to continuously improve quality and processes to meet the changing requirement and expectations of end users. The function of the CCM in relation to this finding relates to the element called health care organisation. This element relates to creating a culture, organisation, and mechanism that promote safe, high quality ART care, including making excellence in ART service provision a priority and pursuing it visibly. 
The third theme discussed in this chapter was ART services that disappointed participants from meeting their requirement. Eight sub-themes were related to and discussed under this theme. Thus, study findings revealed that ART service sites were overstretched; experiencing major space constraints and were aesthetically nonattractive to sight. This finding evolves from failure in delivery system design, one of the six elements of CCM. This element relates to delivering effective, timely and efficient care within an adequate infrastructure. Data analysis also revealed that participants are constantly frustrated with the frequently interrupted laboratory services, arising from frequent equipment breakdown and laboratory reagent stock-out. This finding relates to the CCM element, delivery system design, which refers to delivering effective, timely and efficient laboratory services within an adequate and acceptable infrastructure. The infrastructure includes functional laboratory equipment with available reagents sufficient to carry out tests without interruptions.

It emerged from the study that end users experience inadequate supply and availability of drugs for opportunistic infections and multivitamins. A review of this finding against CCM standards reveals a possibility of weakness in delivery system design. This element of the CCM, delivery system design, could address logistics systems and put a system in place that addresses effective, timely and efficient supplies of OI drugs and multivitamins.

It emanated from this study that end users perceived increasing incidences of failure to treat them with courtesy. Some of them thought this experience could be related to inadequate health care workers and staff burnout of the available few. This finding relates to self-management support, which is an element of the CCM. The element looks at setting a tone of collaboration and professional intimacy between health care workers and end users, to encourage the latter's participation in setting personal goals, adapting self-care and fine-tuning treatment. It also emanated from the study theme that the health facility leadership, policy and strategies are weak in responding to the needs of end users. This finding relates to the decision support element of CCM which uses evidence to influence leadership and policy issues as relevant to ART service provision. This element relates to promoting health facility management and the overall care process, consistent with evidence and end user preferences. 
Findings of the study showed that end users perceive increasing trends of inadequacy in ensuring that they emerge from the health facility healthy. Health measurements are important factors for generating evidence-based data for improving quality of care. In CCM, this element is called clinical information systems. This element relates to organising end users' data to facilitate efficient and effective care. It further emerged from the study that when end users experience challenges in the course of seeking quality ART services in the health facility; they lack guidance on who to contact to lay their complaints or grievances. This indicates that there is inadequate complaints management mechanism in place. Managing complaints requires active participation and coordination between the community and health facility. The component of the CCM that relates to this is community resources. This element relates to mobilising community resources to meet the needs and expectations of end users with long-term conditions such as PLHIV.

Finally on this third theme, findings of the study espoused that health facilities implement sub-optimal standardisation of health care processes and procedures. The CCM element that addresses organisation wide or management related processes and procedure is the health care organisation. This element relates to creating a culture, organisation, and mechanism that promote safe, high quality ART care; and makes excellence in ART service provision a priority, and pursues it visibly.

The fourth and final theme discussed in this chapter was participants' suggestions for improving end users' requirement for quality ART service provision. Four sub-themes were related to and discussed under this theme. Thus, it emanated from the study that appropriate health authorities should deploy, train and re-train additional health care workers to meet the increasing number and dynamic expectations of end users attending ART. The study finding also revealed that HIVIAIDS services should be integrated into regular health services, and end users encouraged on attending the regular clinics. The study finding further highlighted that strengthening supply chain management of laboratory reagents and drugs for opportunistic infections will improve availability and use. Finally, it emerged from the study that health care authorities and service providers should consider reducing frequency of health facility visits by stable end users, in order to reduce end user overload in the ART clinics. 


\section{CHAPTER 6}

\section{CONCLUSION, LIMITATIONS AND RECOMMENDATIONS}

\subsection{INTRODUCTION}

The previous chapter addressed the discussion of findings obtained from focus group discussions. This chapter will focus on drawing conclusions. It will also describe the limitations of the study, and recommendations for improving quality of ART services in Nigerian public health facilities.

The conclusions, based on the research findings discussed in Chapter 5, will be used to answer the research question as stated:

a) What are the perceptions of end users concerning the quality of ART in Nigerian public health facilities?

Finally, the conclusions will ascertain whether the purpose of the study, to develop a best practice guidelines that will promote end user focused quality of ART service provision in public health facilities in Nigeria, which was formulated in section 1.4.1, was achieved.

\subsection{CONCLUSIONS}

The research question was answered by the research findings.

6.2.1 What are the perceptions of end users concerning the quality of ART in Nigerian public health facilities?

According to the perceptions of the participants, only select areas of ART services met their requirement. The results of this study therefore, revealed nine areas of ART services that met end users' requirement. Findings reportedly include: 
- Uninterrupted supplies of ARV drugs improve quality of life.

- $\quad$ Courtesy service of doctors, nurses and other health workers serve as source of encouragement for PLHIV.

- $\quad$ Support group volunteers promote infection prevention and control through triage of vulnerable members in the clinic.

- $\quad$ Enthusiasm of support group volunteers to improve the quality of life of members

- Leadership of support group implement innovative approaches to improve dialogue with health authorities to improve quality of life of people on ART.

- $\quad$ ART knowledge explosion improves cohesion among PLHIV and raises demand for quality of care.

- Volunteer pharmacists improve quality of pharmacy services in ART clinics.

- $\quad$ Empathising with the end user while counselling improves quality of life of the end user.

- $\quad$ Strengthening linkages between support groups and community based organisations provide opportunities for wrap around services and improves the quality of life of PLHIV.

The results of this study revealed that participants indicated nine perceived successes for ART service provision in public health facilities in Nigeria. Findings reportedly include the fact that:

- $\quad$ Health facilities providing ART services have developed linkages with relevant facilities, to obtain specialised diagnostic services, and accept results arising from such facilities.

- Health facilities continuously improve quality and processes to meet the changing requirement and expectations of end users.

- $\quad$ Health facilities continuously improve quality and processes to meet the changing requirement and expectations of end users.

- Health facilities are spacious, clean and attractive, with adequate infrastructure to meet environmental health standards and the aesthetic needs of end users.

- Health facilities have standardised processes that ensure end users spend the least amount of time seeking health care in the facility.

- $\quad$ Government, communities and individuals have joined forces to ensure a stigma free environment for PLHIV. 
- $\quad$ Life saving ART programme is fundamental to the survival of PLHIV, thus, will not experience interruptions.

- Health facilities ensure that any end user who turns up to the health facility benefits from full assessment of his current health status; and is counselled on emerging clinical manifestations that need medical attention.

- Health facilities recognise the essence of PLHIV as volunteers, and design capacity building and motivational programmes to meet both the task requirement and volunteers' expectations.

- Health facilities recognise that PLHIV loves interacting with their peers, and creates opportunities for such interaction as part of the continuum of care process.

The results of this study revealed that participants indicated eight ART service areas that disappointed them from meeting their requirements for quality ART services in public health facilities in Nigeria. Findings reportedly include:

- Weak responsiveness to the needs of end users by the health facility leadership, policy and strategy.

- Overstretched, space constrained and aesthetically non-attractive ART care infrastructure.

- $\quad$ Frequently interrupted laboratory services, related to high equipment down time and commodities stock-out.

- Perceived increasing incidences of failure to treat end users with courtesy, related to inadequate health care workers and staff burnout of the available few.

- Sub-optimal standardisation of health care processes and procedures, with resultant accumulation of bottlenecks along the workflow.

- $\quad$ Perceived increasing trends of inadequacy in ensuring end users emerge from the health facility healthy.

- Inadequate supply and availability of drugs for opportunistic infections and multivitamins.

- $\quad$ Inadequate processes and procedures for managing end users' complaints.

The purpose of the study was achieved. A best practice guideline for the provision of end user focused ART was developed, using the Wagner's chronic care model as a 
framework for its development. This new body of knowledge will be used by health care workers in Nigerian public health facilities to improve end user focused ART.

\subsection{LIMITATIONS OF THE STUDY}

The generalisability of the study's findings is limited by the fact that only 64 end users participated in the FGDs conducted during this study. Therefore, the findings arising from the study may not be generalised beyond the ART clinics of the health facilities studied.

The study might have been limited by biasis that are common to all perception and satisfaction studies. Although proactive actions were taken by the researcher to prevent biasis, the extent and level of accuracy to which these biasis were prevented could not be determined as it was beyond the scope of this study. For example, since the research team were all staff of a project providing ART services in Nigeria, the participants might have responded positively to some questions in order to justify their own time and effort spent obtaining treatment in the health facility. Also, they could have responded negatively in order to attract attention and additional resources for ART to their health facility. Similarly, participants might have given responses because they felt that the responses were more acceptable to satisfy the researchers (social desirability bias). Further more, the participants might have been reluctant of giving negative responses for fear of prejudice from the research team.

Only FGDs were used to collect data. Different results might have been obtained if quantitative structured interviews had been conducted with the end users, and/or if checklists had been completed about observations during ART clinic visits, and/or from ART end users' records. Although these differences could not be pre-determined or projected, it is worthy of mention that satisfaction studies using qualitative methods always produce subjective and challengeable findings. Additionally, perceptions vary depending on the category of people being studied. Thus, the results of this study portray the perceptions of end users who participated in this study, not those of the caregivers or health care workers providing ART services.

Finally, the researcher could have examined each study finding using a combination of two theoretical frameworks (Wagner's chronic care model and Dickoff et al's survey list). 
However, only the Wagner's CCM was used to examine and analyse each study finding, as it was going to be cumbersome and confusing to examine each finding using the combined theoretical frameworks. The Dickoff et al's survey list was a useful framework to developing the user focused best practice guidelines for ART service provision.

\subsection{RECOMMENDATIONS}

\subsubsection{Recommendations for improving quality of ART care in public health facilities}

The following recommendations might improve meeting end users' requirement and expectations for quality ART services in public health facilities in Nigeria:

- Deploy, train and re-train additional skilled personnel to meet increasing number and dynamic expectations of end users in ART sites.

- Integrate HIVIAIDS into regular health services, end users encouraged to attend regular clinics.

- Improve supply chain management of laboratory reagents and drugs for opportunistic infections, to ensure availability and use.

- $\quad$ Reduce end user load in the clinic by reducing frequency of hospital visits by stable end users, through dispensing ARVs that will last 3 months per visit.

\subsubsection{Recommendations for further studies}

- Evaluate the implementation of TQM in a sample of health facilities in Nigeria.

- Quantitative study of the factors of quality of care among users of ART services

- Observational study of the quality of ART services in public health facilities.

- Comparative study of the quality of care in integrated ART clinics versus standalone ART clinics. 


\subsection{CONCLUDING REMARKS}

This study sought to answer this broad question: "What are the perceptions of end users concerning the quality of ART in Nigerian public health facilities?" To answer this broad question, four major themes emerged from the qualitative data analysis on the perceptions of the participants:

- $\quad$ ART services that met participants' requirements.

- $\quad$ Participants' perceived successes in ART service provision

- $\quad$ ART services that disappointed participants from meeting their requirements.

- $\quad$ Participants' suggestions to improve quality of ART service provision.

End users who participated in the study indicated that the following ART services met their expectations: uninterrupted ARV supplies, courtesy treatment of service providers, cohesion and volunteer work of support group members and pharmacists, quality of counselling and linkages between health facility and community based organisations. Participants also shared their perceived successes in ART services provision as follows: ability of ART sites to accept diagnostic results from other facilities, health facilities implement CQI and process improvements, clean and adequate health facility infrastructure, reduced waiting time, stigma reduction, access to comprehensive health assessment and uninterrupted ART services, and collaboration between health care providers and support group to enhance the quality of life of PLHIV. Participants also shared their disappointments regarding ART services provision, thus: weak hospital leadership, non-attractive ART service infrastructure, frequent interrupted laboratory services, inadequate health care workers and staff burnout, long waiting time, inadequacy in ensuring end users emerged from the health facility healthy, interrupted supplies of $\mathrm{Ol}$ drugs and multivitamins and inadequate processes for complaints management. Finally, participants made the following suggestions: deploy and train additional health care workers, integrate ART into regular health services, improve supply chain management of lab reagents and OI drugs, and reduce ART end user overload in the clinics.

In achievement of the overall purpose of the study, a best practice guideline for the provision of end user focused quality ART service provision was developed. The guideline was developed using a combination of survey lists as the conceptual 
framework and chronic care model as the theoretical framework. This new body of knowledge will be used by health care workers in Nigeria and abroad, to improve responsiveness in the provision of quality ART services.

The next chapter (Chapter 7) will present the best practice guidelines for provision of end user focused quality ART service provision in Nigerian public health facilities. 


\section{CHAPTER 7}

\section{BEST PRACTICE GUIDELINES FOR PROVISION OF END USER FOCUSED QUALITY ANTIRETROVIRAL THERAPY IN NIGERIAN PUBLIC HEALTH FACILITIES}

\subsection{INTRODUCTION}

The purpose of this study was to develop best practice guidelines that are user-focused to promote the quality of ART service provision in Nigerian public health facilities. In this chapter the best practice guidelines for provision of end user focused quality ART services in public health facilities are developed. The guidelines are based on the findings from reviewed literature, intuitive insight of the researcher and deductions that combined ideas from several fields of research, including total quality management, nursing and public health. A criterion suggested by Chinn and Kramer (2005:110) was used to validate the guidelines.

In order to attain the purpose of this study the following three objectives should also be addressed:

- Develop a user focused best practice guidelines for ART service provision at Nigerian public health facilities.

- $\quad$ Validate the practice guidelines for ART service provision at Nigerian public health facilities.

- Describe recommendations for implementation of the guidelines for ART service provision at Nigerian public health facilities.

\subsection{PROCESS OF DEVELOPING THE GUIDELINES}

The proposed guidelines were formulated in relation to three major themes that emerged from data analysis on the perceptions of end users concerning the quality of ART in Nigerian public health facilities. 
Two steps were involved in the development of the guideline, composed of considerations of conceptual and theoretical frameworks, respectively. The first step in the development of the guidelines was the consideration of the conceptual framework as elucidated in Chapter 1 of this thesis. The concepts of the framework were applied to provide structure in each of the guidelines. Therefore, included in each guideline was the purpose, the agent, the recipient, context, dynamics and procedures. The second step that the researcher followed in the development of the guidelines was the consideration of the theoretical framework, Wagner's chronic care model, discussed in detail in Chapter 2 of this thesis. The six elements of the CCM were applied to each theme to guide the description of procedures/activities based on their relevance to each element. Therefore, included in each theme were guidelines categorised against the following elements of the CCM: delivery system design, self-management support, decision support, clinical information systems, community resources and health care organisation.

\subsection{APPLICATION OF THE CONCEPTUAL FRAMEWORK TO THE DEVELOPMENT OF THE GUIDELINES}

A survey list consisting of purpose or terminus, the agent, recipient, framework (context), dynamics and the procedures was used as the building block for the guideline (Moleki 2008:162).

\subsubsection{Purpose or terminus}

The purpose of the proposed guidelines is to facilitate staff providing ART touse proven, culturally and linguistically appropriate quality improvement strategies and tools to enable end users understand all treatment options and to make decisions consistent with their values and preferences.

\subsubsection{Agent}

Moleki (2008:163) identified the agent as someone who has the knowledge or ability to perform an activity or provide a solution to a problem. Moleki (2008:163) and Stanhope and Lancaster (2006:215) view an agent as the person who has varying kinds of influence or someone who acts as a precipitating cause of events. In this study an agent 
is a health care provider or professional who is qualified to deliver quality ART in Nigeria. The health care providers include nurses, pharmacists, doctors, laboratory scientists and other paramedical staff.

\subsubsection{Recipient}

The 'recipient' is the beneficiary of the activities designed by the agent. In this study the beneficiaries of quality ART service provision are the end users who are attending ART services in Nigerian public health facilities.

\subsubsection{Framework (context)}

The 'framework' is presented as the context or environment in which activities take place (Moleki 2008:164). This provides for living experiences and it applies to the public health facilities. In this study the lived experiences (perceptions) of end users are explored in public health facilities where ART is provided. These may be subjective as well as objective and are dependent on the dynamic environment in which they are encountered (Moleki 2008:29).

\subsubsection{Dynamics}

The 'dynamics' provide the energy source or the motivating factors for quality of ART (Moleki 2008:165). The dynamics in this study include the availability of ARVs and drugs for opportunistic infections, best pharmacy practices, good laboratory infrastructure and practices, courtesy of health care providers, good documentation and easy retrieval of health records, etc. Good performance of the dynamics results in good end user satisfaction and good health outcomes.

\subsubsection{Procedures}

The 'procedures' according to Moleki (2008:165) are the techniques or protocols that guide the activities. In this study the procedures are the activities presented in accordance with the six elements of the CCM to achieve best practice guidelines that are end user focused. 


\subsection{APPLICATION OF THE THEORETICAL FRAMEWORK TO THE DEVELOPMENT OF THE GUIDELINES}

Wagner's chronic care model was described in Chapter 2, as the theoretical framework used in this study. The CCM acknowledges that a substantial portion of chronic care takes place outside formal health care settings and suggests that six elements are of central importance in initiatives to improve chronic care: community resources; the health care system; end user self-management; decision support; delivery system redesign; and clinical information systems (Singh \& Ham 2006:5). The elements of the model, based on research evidence, are presented in a tabular matrix below, linking research findings with recommended procedures/activities for the best practice guidelines for ART service provision in Nigerian public health facilities.

The tabular matrix below shows the themes that emerged from the study, a list of findings against each theme, the element of Wagner's CCM that relates to each finding, and the recommended procedures or activities for the implementation of the finding.

Table 7.1 Matrix showing best practice guidelines for provision of end user focused quality ART service provision in public health facilities

\begin{tabular}{|c|c|c|c|}
\hline Theme & Findings from the study & $\begin{array}{l}\text { Elements of } \\
\text { Wagner's } \\
\text { Chronic Care } \\
\text { Model }\end{array}$ & $\begin{array}{l}\text { Recommended } \\
\text { procedures/activities for } \\
\text { implementation of the guideline }\end{array}$ \\
\hline $\begin{array}{l}\text { Guideline to address } \\
\text { provision of ART } \\
\text { services that meet } \\
\text { end users' } \\
\text { requirement and } \\
\text { expectations }\end{array}$ & $\begin{array}{l}\text { 1. Uninterrupted supply of } \\
\text { ARV drugs could } \\
\text { improve quality of life }\end{array}$ & $\begin{array}{l}\text { Delivery system } \\
\text { design }\end{array}$ & $\begin{array}{l}\text { 1. Ensure uninterrupted supply of } \\
\text { antiretroviral drugs at all times } \\
\text { Carry out adequate drug } \\
\text { forecasting and quantification } \\
\text { annually, and review drug } \\
\text { pipeline at least, quarterly. } \\
\text { Maintain adequate and accurate } \\
\text { drug logistics data, and fill supply } \\
\text { gaps well in advance, where } \\
\text { necessary. } \\
\text { Strengthen linkages with other } \\
\text { health facilities or programmes } \\
\text { providing ART, to loan drugs } \\
\text { from, whenever in emergency } \\
\text { need }\end{array}$ \\
\hline & $\begin{array}{l}\text { 2. Courtesy service of } \\
\text { doctors, nurses and } \\
\text { other health workers }\end{array}$ & $\begin{array}{l}\text { Self-management } \\
\text { support }\end{array}$ & $\begin{array}{l}\text { 2. Consider all end users as fellow } \\
\text { human beings, set a tone of } \\
\text { collaboration and participation, }\end{array}$ \\
\hline
\end{tabular}




\begin{tabular}{|c|c|c|c|}
\hline Theme & Findings from the study & $\begin{array}{l}\text { Elements of } \\
\text { Wagner's } \\
\text { Chronic Care } \\
\text { Model }\end{array}$ & $\begin{array}{l}\text { Recommended } \\
\text { procedures/activities for } \\
\text { implementation of the guideline }\end{array}$ \\
\hline & $\begin{array}{l}\text { serve as source of } \\
\text { encouragement for } \\
\text { PLHIV } \\
\text { 3. Empathising with the } \\
\text { end user while } \\
\text { counselling improves } \\
\text { quality of life of the end } \\
\text { user } \\
\text { 4. ART knowledge } \\
\text { explosion improves } \\
\text { cohesion among PLHIV } \\
\text { and raises demand for } \\
\text { quality of care }\end{array}$ & & $\begin{array}{l}\text { and use a collective team } \\
\text { approach to treat end users with } \\
\text { courtesy. Encourage end users } \\
\text { through communication and } \\
\text { interpersonal relationship. Instill a } \\
\text { spiritual connection in dealing } \\
\text { with end users, by giving due } \\
\text { recognition to his/her religious } \\
\text { affiliation. } \\
\text { Empathise with the end users } \\
\text { while counselling and providing } \\
\text { ART services, to open } \\
\text { communication and improve } \\
\text { quality of life of the end users. } \\
\text { Maintain high level of trust } \\
\text { between health care worker and } \\
\text { end users, to sustain confidence } \\
\text { in quality of counselling services. } \\
\text { Provide ART education to end } \\
\text { users to widen their knowledge } \\
\text { base and encourage their } \\
\text { participation in setting goals and } \\
\text { fine-tuning their treatment. } \\
\text { Maintain continued active } \\
\text { involvement of the end users in } \\
\text { their care. Train end users as } \\
\text { expert users to educate their } \\
\text { peers on issues related to } \\
\text { HIVIAIDS, including ART. } \\
\text { Encourage group dialogue, } \\
\text { decision-making and reflections } \\
\text { among peers, on improved ways } \\
\text { of ensuring self-care. Encourage } \\
\text { self-care innovations generated } \\
\text { by support groups. }\end{array}$ \\
\hline & $\begin{array}{l}\text { 5. Support group } \\
\text { volunteers are } \\
\text { enthusiastic of improving } \\
\text { the quality of life of their } \\
\text { members } \\
\text { 6. Support group } \\
\text { volunteers promote } \\
\text { infection prevention and } \\
\text { control through triage of } \\
\text { vulnerable members in } \\
\text { the clinic }\end{array}$ & Decision support & $\begin{array}{l}\text { 5. Encourage health facility } \\
\text { management to institutionalise } \\
\text { support group volunteerism in the } \\
\text { health facility. Encourage end } \\
\text { users to voluntarily collaborate } \\
\text { and participate in activities } \\
\text { leading to improving the quality } \\
\text { of life of other end users } \\
\text { attending ART services. Identify } \\
\text { individual interests of end users } \\
\text { and train them on income } \\
\text { generating activities/skills } \\
\text { acquisition. Trained end users } \\
\text { should be encouraged to train } \\
\text { their peers. Geographically } \\
\text { delineate end users into } \\
\text { community clusters and assign } \\
\text { support group volunteers to take } \\
\text { responsibility for follow up and } \\
\text { contact tracking of end users in } \\
\text { each manageable cluster. Train }\end{array}$ \\
\hline
\end{tabular}




\begin{tabular}{|c|c|c|c|}
\hline \multirow[t]{2}{*}{ Theme } & Findings from the study & \multirow[t]{2}{*}{$\begin{array}{l}\text { Elements of } \\
\text { Wagner's } \\
\text { Chronic Care } \\
\text { Model }\end{array}$} & $\begin{array}{l}\text { Recommended } \\
\text { procedures/activities for } \\
\text { implementation of the guideline }\end{array}$ \\
\hline & & & $\begin{array}{l}\text { and engage volunteers to close } \\
\text { health care worker gaps in the } \\
\text { health facility, carrying out basic } \\
\text { non-high technical functions. } \\
\text { 6. Capacitate members of the } \\
\text { support group of PLHIV to serve } \\
\text { as volunteers, promoting } \\
\text { infection prevention and control } \\
\text { through triage of vulnerable end } \\
\text { users in the ART clinic }\end{array}$ \\
\hline & $\begin{array}{l}\text { 7. Volunteer pharmacists } \\
\text { improve quality of } \\
\text { pharmacy services in } \\
\text { ART clinics }\end{array}$ & $\begin{array}{l}\text { Clinical } \\
\text { information } \\
\text { systems }\end{array}$ & $\begin{array}{l}\text { 7. Utilise volunteer professional } \\
\text { pharmacists, where dearth of } \\
\text { health care workers exists in the } \\
\text { pharmacy; to improve ARV } \\
\text { dispensing, recording and } \\
\text { reporting, and overall quality of } \\
\text { pharmacy services in the ART } \\
\text { clinic. Volunteer Pharmacists } \\
\text { should join the regular } \\
\text { pharmacists in providing user } \\
\text { focused pharmacy services. }\end{array}$ \\
\hline & $\begin{array}{l}\text { 8. Strengthening linkages } \\
\text { between support groups } \\
\text { and community based } \\
\text { organisations provide } \\
\text { opportunities for wrap } \\
\text { around services and } \\
\text { improves the quality of } \\
\text { life of PLHIV }\end{array}$ & $\begin{array}{l}\text { Community } \\
\text { resources }\end{array}$ & $\begin{array}{l}\text { 8. Strengthen coherent system } \\
\text { improvements that promote } \\
\text { linkages between health facilities } \\
\text { and faith- and community based } \\
\text { organisations to expand access } \\
\text { to services that are not } \\
\text { obtainable in the health facility } \\
\text { setting. These services include } \\
\text { income generating activities, food } \\
\text { and nutrition, spiritual care, etc. } \\
\text { Strengthen linkages between } \\
\text { community pharmacies and } \\
\text { health facilities to support } \\
\text { continuum of care for PLHIV. }\end{array}$ \\
\hline & $\begin{array}{l}\text { 9. } \\
\text { groadership of support } \\
\text { innovative approaches to } \\
\text { improve dialogue with } \\
\text { health authorities to } \\
\text { improve quality of life of } \\
\text { end users attending ART }\end{array}$ & $\begin{array}{l}\text { Health care } \\
\text { organisation }\end{array}$ & $\begin{array}{l}\text { 9. Strengthen relationships between } \\
\text { the leadership of support group } \\
\text { of PLHIV and the health facility } \\
\text { management, to improve } \\
\text { communication/dialogue, } \\
\text { collaboration and participation in } \\
\text { decisions regarding the welfare } \\
\text { and other needs of end users } \\
\text { attending ART. Health facility } \\
\text { leadership should understand } \\
\text { that end users are the purpose } \\
\text { for their existence in the facility, } \\
\text { and do everything humanly } \\
\text { possible to meet their needs. } \\
\text { Support group leadership should } \\
\text { mediate between end users and } \\
\text { health facility leadership, and } \\
\text { monitor implementation of any } \\
\text { action points reached at all } \\
\text { meetings held with health facility }\end{array}$ \\
\hline
\end{tabular}




\begin{tabular}{|c|c|c|c|}
\hline \multirow[t]{2}{*}{ Theme } & \multirow[t]{2}{*}{ Findings from the study } & \multirow{2}{*}{$\begin{array}{l}\text { Elements of } \\
\text { Wagner's } \\
\text { Chronic Care } \\
\text { Model }\end{array}$} & $\begin{array}{l}\text { Recommended } \\
\text { procedures/activities for } \\
\text { implementation of the guideline }\end{array}$ \\
\hline & & & management. \\
\hline $\begin{array}{l}\text { Guideline to maintain } \\
\text { successes of ART }\end{array}$ & $\begin{array}{l}\text { 1. Health facilities are } \\
\text { spacious, clean and } \\
\text { attractive, with adequate } \\
\text { infrastructure to meet } \\
\text { environmental health } \\
\text { standards and the } \\
\text { aesthetic needs of end } \\
\text { users } \\
\text { 2. Lifesaving ART } \\
\text { programme is } \\
\text { fundamental to the } \\
\text { survival of PLHIV, thus, } \\
\text { will not experience } \\
\text { interruptions. } \\
\text { Health facilities ensure } \\
\text { that any end user who } \\
\text { turns up to the hospital } \\
\text { benefits from full } \\
\text { assessment of his } \\
\text { current health status; } \\
\text { and is counselled on } \\
\text { emerging clinical } \\
\text { manifestations that } \\
\text { needs medical attention. }\end{array}$ & $\begin{array}{l}\text { Delivery system } \\
\text { design }\end{array}$ & 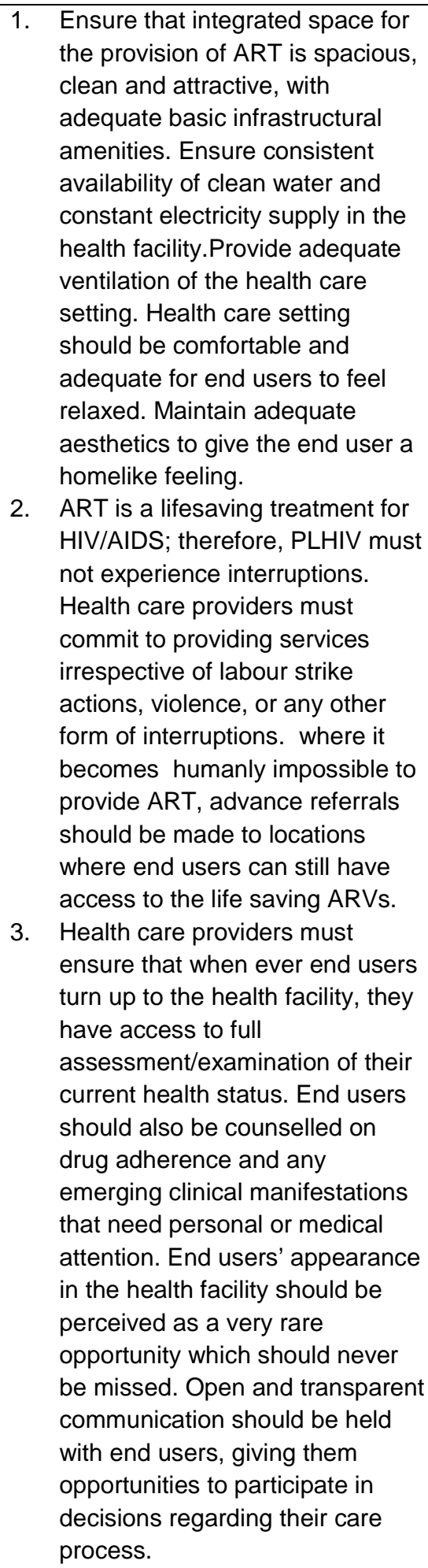 \\
\hline & $\begin{array}{l}\text { 4. Health facilities } \\
\text { recognise the essence of } \\
\text { PLHIV as volunteers, } \\
\text { and design capacity } \\
\text { building and motivational } \\
\text { programmes to meet } \\
\text { both the task }\end{array}$ & $\begin{array}{l}\text { Self-management } \\
\text { support }\end{array}$ & $\begin{array}{l}\text { 4. Health care providers should } \\
\text { understand that end users are } \\
\text { capable of making decisions } \\
\text { regarding their care, and can } \\
\text { actually carry out some basic } \\
\text { care themselves, as well as care } \\
\text { for their fellow end users. }\end{array}$ \\
\hline
\end{tabular}




\begin{tabular}{|c|c|c|c|}
\hline Theme & Findings from the study & $\begin{array}{l}\text { Elements of } \\
\text { Wagner's } \\
\text { Chronic Care } \\
\text { Model }\end{array}$ & $\begin{array}{l}\text { Recommended } \\
\text { procedures/activities for } \\
\text { implementation of the guideline }\end{array}$ \\
\hline & $\begin{array}{l}\text { requirement and } \\
\text { volunteers' expectations. }\end{array}$ & & $\begin{array}{l}\text { Volunteers should be motivated } \\
\text { through capacity building to } \\
\text { empower them to be self } \\
\text { sufficient and also support their } \\
\text { peers. Programmes should } \\
\text { provide facilitation and logistics } \\
\text { support to PLHIV support groups } \\
\text { to enhance their performance. } \\
\text { Programme managers/health } \\
\text { facility authorities should facilitate } \\
\text { support group's access to } \\
\text { community gate keepers, to } \\
\text { access support for their group } \\
\text { members. Equip support group } \\
\text { volunteers to carry out self- } \\
\text { management: they require self- } \\
\text { management assessment tools, } \\
\text { ART education tools, self- } \\
\text { management resources and tools } \\
\text { (including guidelines for self- } \\
\text { care), psychosocial support and } \\
\text { opportunities for on-going } \\
\text { collaborative decision-making } \\
\text { regarding their care. }\end{array}$ \\
\hline & $\begin{array}{l}\text { 5. Health facilities } \\
\text { providing ART services } \\
\text { have developed linkages } \\
\text { with relevant facilities, to } \\
\text { obtain specialised } \\
\text { diagnostic services, and } \\
\text { accept results arising } \\
\text { from such facilities. }\end{array}$ & Decision support & $\begin{array}{l}\text { 5. No single health facility is } \\
\text { equipped to provide all arrays of } \\
\text { services without dependence on } \\
\text { another facility. Therefore, health } \\
\text { care providers should identify } \\
\text { possibilities of networking with } \\
\text { other facilities and strengthen } \\
\text { linkages with available networks } \\
\text { of both basic and specialised } \\
\text { health services. Where a } \\
\text { strengthened network involves } \\
\text { laboratories, health care } \\
\text { providers must trust the } \\
\text { capabilities of their sister facilities } \\
\text { and accept diagnostic results } \\
\text { issued from such health facilities. } \\
\text { Networks should also include } \\
\text { opportunities for expert } \\
\text { consultations, where a case } \\
\text { cannot be managed with } \\
\text { resources available in the } \\
\text { source/primary health facility. } \\
\text { Programme managers should } \\
\text { make provision for back-up } \\
\text { laboratory equipment to avoid } \\
\text { interruptions owing to equipment } \\
\text { breakdown. }\end{array}$ \\
\hline & $\begin{array}{l}\text { 6. Health facilities has } \\
\text { standardised processes } \\
\text { that ensures end users } \\
\text { spend the least amount } \\
\text { of time seeking health }\end{array}$ & $\begin{array}{l}\text { Clinical } \\
\text { information } \\
\text { systems }\end{array}$ & $\begin{array}{l}\text { 6. Health facilities should invest in } \\
\text { standardising processes that } \\
\text { ensures end users spend the } \\
\text { least amount of time seeking } \\
\text { health care in the facility. Target }\end{array}$ \\
\hline
\end{tabular}




\begin{tabular}{|c|c|c|c|}
\hline Theme & Findings from the study & $\begin{array}{l}\text { Elements of } \\
\text { Wagner's } \\
\text { Chronic Care } \\
\text { Model }\end{array}$ & $\begin{array}{l}\text { Recommended } \\
\text { procedures/activities for } \\
\text { implementation of the guideline }\end{array}$ \\
\hline & care in the facility & & $\begin{array}{l}\text { the pharmacy and medical } \\
\text { records units/departments for } \\
\text { intensive process improvement } \\
\text { measures, due to usual long } \\
\text { waiting time in these work } \\
\text { stations. Involve end users in } \\
\text { developing process improvement } \\
\text { initiatives that are aimed at } \\
\text { improving efficiency and } \\
\text { effectiveness. Where practicable, } \\
\text { automation of processes such as } \\
\text { medical records is encouraged, } \\
\text { to reduce waiting time. The } \\
\text { health facility should invest in } \\
\text { computerised end user records, } \\
\text { with special software to organise } \\
\text { data and alert members of the } \\
\text { care team to end users' needs. } \\
\text { The health care providers can } \\
\text { measure their performance } \\
\text { against quality benchmarks set } \\
\text { out in the software. Through } \\
\text { management by facts/evidence } \\
\text { end users are healthier, health } \\
\text { care providers are cheered and } \\
\text { motivated by evidence of a job } \\
\text { well done, and the health care } \\
\text { system saves money. }\end{array}$ \\
\hline & 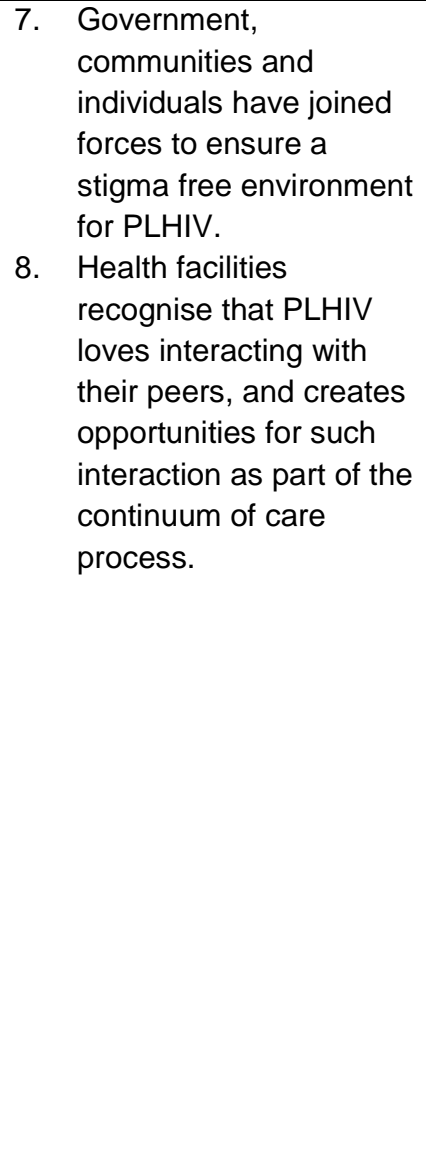 & $\begin{array}{l}\text { Community } \\
\text { resources }\end{array}$ & $\begin{array}{l}\text { 7. Strengthen partnerships between } \\
\text { the government, communities, } \\
\text { civil society groups and } \\
\text { individuals to fight stigma, and } \\
\text { create a stigma free } \\
\text { environment, necessary for the } \\
\text { quality care of ART. Government } \\
\text { should support stigma reduction } \\
\text { mass media campaigns, while } \\
\text { civil societies and health facilities } \\
\text { should intensify on targeted } \\
\text { stigma reduction programmes. } \\
\text { Health care workers should be } \\
\text { trained and sensitised around } \\
\text { professional ethics, issues of } \\
\text { confidentiality and stigma } \\
\text { reduction. Programme managers } \\
\text { should consider and ensure end } \\
\text { users' audio-visual privacy when } \\
\text { designing or allocating points of } \\
\text { service in health facilities. } \\
\text { Support a healthy environment } \\
\text { where PLHIV socialise with their } \\
\text { peers and collaborate to help } \\
\text { each other as part of the } \\
\text { continuum of care process. } \\
\text { Encourage formation of small } \\
\text { groups of } 4-8 \text { PLHIV each, to }\end{array}$ \\
\hline
\end{tabular}




\begin{tabular}{|c|c|c|c|}
\hline Theme & Findings from the study & $\begin{array}{l}\text { Elements of } \\
\text { Wagner's } \\
\text { Chronic Care }\end{array}$ & $\begin{array}{l}\text { Recommended } \\
\text { procedures/activities for } \\
\text { implementation of the guideline }\end{array}$ \\
\hline & & & $\begin{array}{l}\text { serve as self-care quality } \\
\text { improvement collaborative and } \\
\text { treatment support to each other. } \\
\text { The group will work together to } \\
\text { dialogue, reflect, learn and } \\
\text { consistently develop and } \\
\text { implement innovative } \\
\text { approaches in self-care. They will } \\
\text { also assist each other in carrying } \\
\text { out activities which they could not } \\
\text { do by themselves anylonger. }\end{array}$ \\
\hline & $\begin{array}{l}\text { 9. Health facilities } \\
\text { continuously improve } \\
\text { quality and processes to } \\
\text { meet the changing } \\
\text { requirement and } \\
\text { expectations of end } \\
\text { users. }\end{array}$ & $\begin{array}{l}\text { Health care } \\
\text { organisation }\end{array}$ & $\begin{array}{l}\text { 9. Establish an end user driven } \\
\text { quality-based culture and } \\
\text { framework for quality } \\
\text { improvement in the health facility. } \\
\text { The quality framework should } \\
\text { have a unifying purpose } \\
\text { (mission), vision and a strategy } \\
\text { to sustain it. } \\
\text { Secure commitment of top } \\
\text { leadership to continuously } \\
\text { improve quality and processes to } \\
\text { meet the changing requirement } \\
\text { and expectations of end users. } \\
\text { Institute measures to prevent } \\
\text { extortion of end users by } \\
\text { anybody, and to protect the rights } \\
\text { of the individual end user. } \\
\text { Automate systems such as } \\
\text { medical records systems, where } \\
\text { feasible, to reduce end user } \\
\text { waiting time.Segment and } \\
\text { decentralise points of services, } \\
\text { especially, medical records and } \\
\text { pharmacy sections, to increase } \\
\text { speedy access to end users. } \\
\text { Where feasible, gainfully engage } \\
\text { support group volunteers to fill } \\
\text { human resource gaps in the } \\
\text { health facility. Programme } \\
\text { managers should ensure } \\
\text { uninterrupted supplies of health } \\
\text { commodities, including nutritional } \\
\text { supplements, drugs, and where } \\
\text { feasible, link end users to food } \\
\text { sources. } \\
\text { Support the integration of } \\
\text { HIVIAIDS into general health } \\
\text { care services, improve efficiency } \\
\text { and effectiveness in care, reduce } \\
\text { stigma and re-inforce end user } \\
\text { rapport with health care workers. }\end{array}$ \\
\hline $\begin{array}{l}\text { Guideline to address } \\
\text { ART services that }\end{array}$ & $\begin{array}{l}\text { 1. Overstretched, space } \\
\text { constrained and }\end{array}$ & $\begin{array}{l}\text { Delivery system } \\
\text { design }\end{array}$ & $\begin{array}{l}\text { 1. Reduce end user load in the } \\
\text { clinic by reducing frequency of }\end{array}$ \\
\hline
\end{tabular}




\begin{tabular}{|c|c|c|c|}
\hline Theme & Findings from the study & $\begin{array}{l}\text { Elements of } \\
\text { Wagner's } \\
\text { Chronic Care } \\
\text { Model }\end{array}$ & $\begin{array}{l}\text { Recommended } \\
\text { procedures/activities for } \\
\text { implementation of the guideline }\end{array}$ \\
\hline $\begin{array}{l}\text { failed to meet end } \\
\text { users' requirement } \\
\text { and expectations }\end{array}$ & 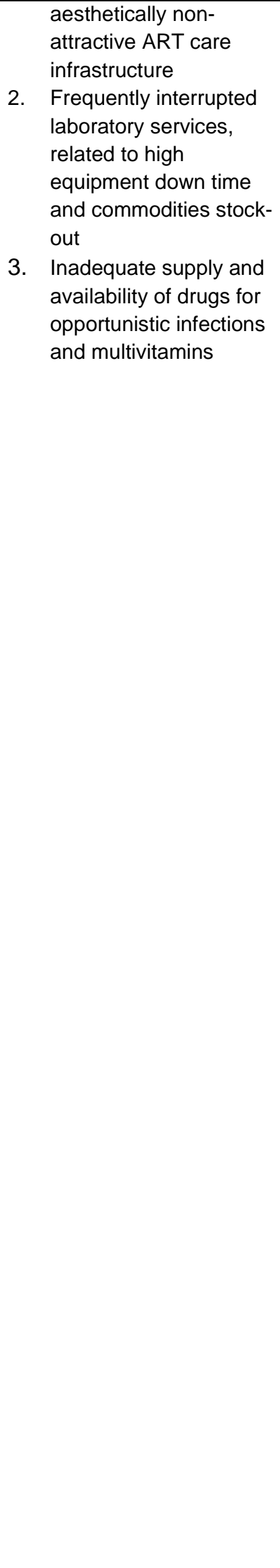 & & 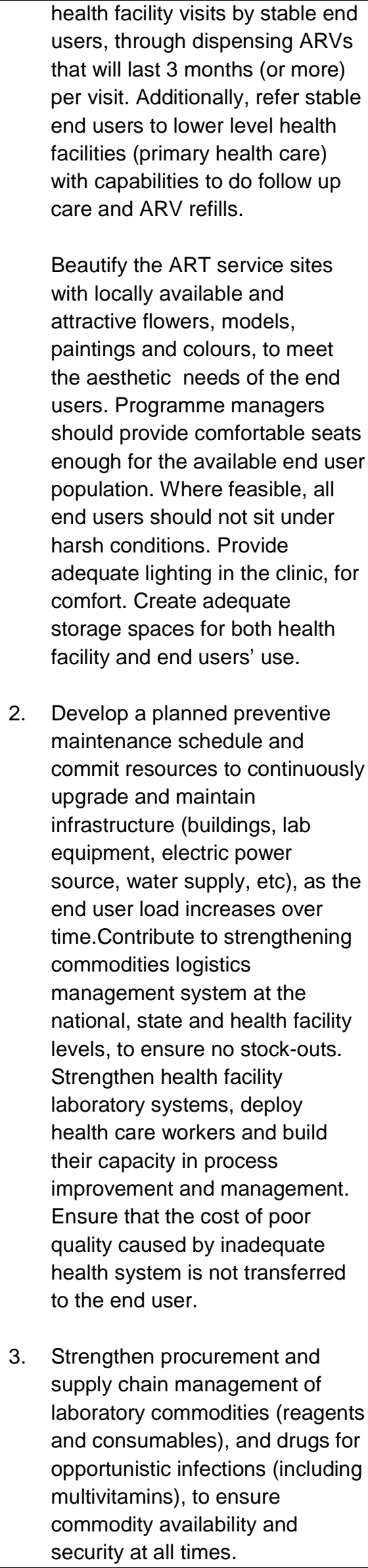 \\
\hline
\end{tabular}




\begin{tabular}{|c|c|c|c|}
\hline Theme & Findings from the study & $\begin{array}{l}\text { Elements of } \\
\text { Wagner's } \\
\text { Chronic Care } \\
\text { Model }\end{array}$ & $\begin{array}{l}\text { Recommended } \\
\text { procedures/activities for } \\
\text { implementation of the guideline }\end{array}$ \\
\hline & $\begin{array}{l}\text { 4. } \\
\text { incidenceived increasing } \\
\text { treat end users with } \\
\text { courtesy, related to } \\
\text { inadequate health care } \\
\text { workers and staff } \\
\text { burnout of the available } \\
\text { few. }\end{array}$ & $\begin{array}{l}\text { Self-management } \\
\text { support }\end{array}$ & $\begin{array}{l}\text { 4. Conduct workflow and workload } \\
\text { analysis of the ART clinic to } \\
\text { determine work stations requiring } \\
\text { additional health care } \\
\text { worker/capacity, to prevent } \\
\text { health care worker burnout. } \\
\text { Deploy, train and re-train } \\
\text { additional health care workers on } \\
\text { CCM of care of chronic illnesses, } \\
\text { to meet the increasing needs and } \\
\text { dynamic expectations of end } \\
\text { users attending ART. } \\
\text { Analyse training needs and train } \\
\text { health care workers on } \\
\text { interpersonal communication and } \\
\text { counselling. Institute measures } \\
\text { (with sanctions - performance } \\
\text { issues) for discrimination, } \\
\text { intimidation and or scolding of } \\
\text { end users by health care } \\
\text { workers. } \\
\text { Train end users to take on basic } \\
\text { self-care responsibilities to } \\
\text { lessen burden on health care } \\
\text { providers. } \\
\text { Strengthen the use of clinic } \\
\text { diaries to schedule appointments } \\
\text { for end users' clinic attendance, } \\
\text { based on established results of } \\
\text { workflow and workload analysis } \\
\text { in the clinic. }\end{array}$ \\
\hline & $\begin{array}{l}\text { 5. Weak responsiveness to } \\
\text { the needs of end users } \\
\text { by the hospital } \\
\text { leadership, policy and } \\
\text { strategy }\end{array}$ & Decision support & $\begin{array}{l}\text { 5. Health facility leadership should } \\
\text { recognise that end users are the } \\
\text { reasons for their existence in the } \\
\text { health facility. They should seek } \\
\text { and exercise adequate authority } \\
\text { to ensure the protection of end } \\
\text { users and ensure that they leave } \\
\text { the health facility healthy. } \\
\text { End users depend on health care } \\
\text { workers' output; therefore, failure } \\
\text { to meet or exceed their } \\
\text { expectations would ultimately } \\
\text { result in the loss of end user } \\
\text { loyalty and patronage and/or may } \\
\text { cost them their jobs, if the voice } \\
\text { of the end user is ultimately } \\
\text { heard by the authorities. }\end{array}$ \\
\hline & $\begin{array}{l}\text { 6. } \\
\text { trends of inadequacy in } \\
\text { ensuring end users } \\
\text { emerge from the health }\end{array}$ & $\begin{array}{l}\text { Clinical } \\
\text { information } \\
\text { systems }\end{array}$ & $\begin{array}{l}\text { 6. Establish a system to } \\
\text { continuously measure, report on, } \\
\text { and improve the quality of ART } \\
\text { from the perspectives of end }\end{array}$ \\
\hline
\end{tabular}




\begin{tabular}{|c|c|c|c|}
\hline Theme & Findings from the study & $\begin{array}{l}\text { Elements of } \\
\text { Wagner's } \\
\text { Chronic Care } \\
\text { Model }\end{array}$ & $\begin{array}{l}\text { Recommended } \\
\text { procedures/activities for } \\
\text { implementation of the guideline }\end{array}$ \\
\hline & facility healthy & & $\begin{array}{l}\text { users. Examples include } \\
\text { measures of timeliness (getting } \\
\text { ART as soon as wanted); } \\
\text { measures of poor communication } \\
\text { at the health facility providing } \\
\text { ART (end user's assessments of } \\
\text { how often their provider listened } \\
\text { carefully to them, explained } \\
\text { things clearly, respected what } \\
\text { they had to say, and spent } \\
\text { enough time with them); and } \\
\text { measures of end user safety } \\
\text { (health care-associated } \\
\text { infections, serious adverse } \\
\text { effects, etc). } \\
\text { Health care providers should put } \\
\text { in place systems to monitor } \\
\text { trends of the performance } \\
\text { measures described above, and } \\
\text { ensure end users emerge from } \\
\text { the health facility healthy. }\end{array}$ \\
\hline & $\begin{array}{l}\text { 7. Inadequate processes } \\
\text { and procedures for } \\
\text { managing end users' } \\
\text { complaints }\end{array}$ & $\begin{array}{l}\text { Community } \\
\text { resources }\end{array}$ & $\begin{array}{l}\text { 7. Understand the socio-cultural } \\
\text { values of the host community } \\
\text { and end users' perceptions of the } \\
\text { role of the health system, and } \\
\text { then, bring health care providers } \\
\text { and representatives of the } \\
\text { community together to map out a } \\
\text { complaints management system } \\
\text { for the health facility. } \\
\text { Engage a team composed of end } \\
\text { users, community stakeholders } \\
\text { and health care providers to } \\
\text { monitor, measure, report on, } \\
\text { provide feedback and improve on } \\
\text { the management of end users' } \\
\text { complaints. } \\
\text { Encourage the use of statistical } \\
\text { thinking in the process of } \\
\text { complaints management. It is } \\
\text { management by evidence/facts } \\
\text { (not opinion, guesswork or gut } \\
\text { feel) that can reinforce trust and } \\
\text { loyalty in the health facility, by } \\
\text { the community. }\end{array}$ \\
\hline & $\begin{array}{l}\text { 8. Sub-optimal } \\
\text { standardisation of health } \\
\text { care processes and } \\
\text { procedures, with } \\
\text { resultant accumulation of } \\
\text { bottlenecks along the } \\
\text { workflow }\end{array}$ & $\begin{array}{l}\text { Health care } \\
\text { organisation }\end{array}$ & $\begin{array}{l}\text { 8. Establish a system to } \\
\text { continuously measure, report on } \\
\text { and improve health care } \\
\text { processes and procedures, } \\
\text { seeking substantial inputs from } \\
\text { end users, to ensure efficiency } \\
\text { and effectiveness through } \\
\text { removal of unnecessary }\end{array}$ \\
\hline
\end{tabular}




\begin{tabular}{|c|c|c|c|}
\hline Theme & Findings from the study & $\begin{array}{l}\text { Elements of } \\
\text { Wagner's } \\
\text { Chronic Care } \\
\text { Model }\end{array}$ & $\begin{array}{l}\text { Recommended } \\
\text { procedures/activities for } \\
\text { implementation of the guideline }\end{array}$ \\
\hline & & & $\begin{array}{l}\text { redundancies, duplication of } \\
\text { effort and bottlenecks. } \\
\text { Develop and train staff to use } \\
\text { standard operating procedures } \\
\text { (SOP) for all processes that are } \\
\text { commonly recurring in the health } \\
\text { facility. Constantly obtain } \\
\text { feedback from end users to } \\
\text { update the SOP from time to } \\
\text { time, ensuring it constantly meets } \\
\text { their expectations. } \\
\text { Health facility leaders should } \\
\text { focus on processes rather than } \\
\text { people as the source of problems } \\
\text { in the health care system. }\end{array}$ \\
\hline
\end{tabular}

\subsection{FORMULATION OF BEST PRACTICE GUIDELINES FOR PROVISION OF END USER FOCUSED QUALITY ANTIRETROVIRAL THERAPY IN NIGERIAN PUBLIC HEALTH FACILITIES}

This section presents guidelines for the health care workers based on synthesis of the researcher's conclusions drawn from the study findings, intuitions and results of literature search. Formulation of the guidelines is also guided by the conceptual and theoretical frameworks used in this study. Sets of guidelines were developed from each theme, in line with the sub-themes that emerged from the themes, and as guided by the elements of the Wagner's CCM.

The guidelines are presented under each theme that emerged from this study.

\subsubsection{THEME 1: Guidelines to address provision of ART services that meet end users' requirements}

Nine guidelines were developed from this theme, arising from nine sub-themes that emerged from the theme. Each set of guidelines are preceded by an objective and appears under the appropriate element of Wagner's CCM. 


\subsubsection{Delivery system design}

a. To ensure uninterrupted supply of antiretroviral drugs at all times

o Carry out adequate drug forecasting and quantification annually, and review drug pipeline at least, quarterly.

o Maintain adequate and accurate drug logistics data, and fill supply gaps well in advance, where necessary.

o Strengthen linkages with other health facilities or programmes providing ART, to loan drugs from, whenever in emergency stock out situation or in urgent need.

\subsubsection{Self-management support}

b. To consider all end users as fellow human beings

o Set a tone of collaboration and participation with the end users and use a collective team approach to treat end users with courtesy.

o Listen carefully to what end users want to say, explain things clearly and treat them with courtesy and respect.

o Actively engage end users in their care.

o Empower end users to effectively navigate and manage their care.

o Empower end users to make informed decisions about their treatment options.

o The decision to initiate ART and/or to select antiretroviral agents should be individualised for each end user and incorporate assessment of the following factors: risk of progression to illness or death if untreated; readiness and willingness to adhere to therapy; potential barriers to adherence; comorbidities and coexisting conditions; risk of HIV transmission to others if untreated; and risk of toxicities and drug-drug interactions.

o Clinicians should involve the end user when planning treatment and when deciding which antiretroviral regimen is most likely to result in adherence; and the end user should make the final decision of whether and when to initiate ART. 
o Encourage end users through effective communication and interpersonal relationship.

o Instill a spiritual connection in dealing with end users, by giving due recognition to his/her religious affiliation.

c. To empathise with end user while counselling and providing ART services

o Engage in open communication and transparent communication with end users.

o Initiate good provider-end user communication so that end users' needs are understood and addressed. Demonstrate understanding that effective communication facilitates end user participation in their care.

o Show compassion, empathy, and responsiveness to the needs, values, and expressed preferences of the individual end user.

o View the end user as a unique person, rather than focusing strictly on the illness.

o Build a therapeutic alliance based on the end user's and the health provider's perspectives.

o Translate and interpret where language barriers exist between the end user and his/her health provider, as this is a legal requirement.

o Except when initiation of treatment is clinically urgent, clinicians should use more than one visit for education and counselling before committing an end user to a specific therapy. Counselling and education should include the following: available treatment options, potential benefits of therapy, risks of therapy, the need for strict adherence and the risk of viral drug resistance when adherence is suboptimal. The use of safer-sex practices and avoidance of needle-sharing activity should be addressed regardless of viral load, to prevent HIV transmission or superinfection.

o Maintain high level of trust between health care worker and end user, to sustain confidence in quality of counselling services. 
d. To provide ART education to end users to widen their knowledge base and encourage their participation in setting goals and fine-tuning their treatment

o Provide ART service information in simple words, and confirm that end users have understood the information given.

o Provide correct and accurate information regarding ART, when end users are discharged home.

o Maintain continued active involvement of the end users in their care. Train end users as expert users to educate their peers on issues related to HIVIAIDS, including ART.

- Encourage group dialogue, decision-making and reflections among peers, on improved ways of ensuring self-care. Encourage self-care innovations generated by support groups.

\subsubsection{Decision support}

e. To institutionalise the use of members of the support group of PLHIV to serve as volunteers in ART services

o Health facility leadership should recognise the existence of PLHIV volunteers in the health facility.

o Train and mentor PLHIV to promote infection prevention and control in ART service areas.

o Empower and position PLHIV to carry out basic functions in the ART clinic, including but not limited to adherence counselling, triage, sorting, retrieval and replacement of end user ART folders/records, health and treatment education, etc.

o Identify individual interests of end users and train them on income generating activities/skills acquisition.

o Trained end users should be encouraged to train others (their peers).

o Geographically delineate end users into community clusters and assign support group volunteers to take responsibility for follow up and contact tracking of end users in each manageable cluster.

o Train and engage volunteers to close health care worker gaps in the health facility, carrying out basic none-high technical functions. 


\subsubsection{Clinical information system}

f. To improve pharmacy documentation and practices

o Utilise volunteer professional pharmacists to improve ARV dispensing, recording and reporting, and overall quality of pharmacy services in the ART clinic

o Volunteer Pharmacists should join the regular pharmacists in providing user focused pharmacy services.

o Where a dearth of pharmacy staff exists, volunteer pharmacists should be solicited to assist regular pharmacists in updating pharmacy records/backlogs, where such exist and maintain accurate and up to date pharmacy records at all times.

\subsubsection{Community resources}

g. To encourage end users to voluntarily collaborate and participate in activities leading to improving the quality of life of other end users attending ART services

o Establish a system of PLHIV facilitated group learning experiences that deal with ART from the perspectives of end users. A health care provider should initiate and mentor end users to be group leaders.

o Encourage home visits for PLHIV by the PLHIV.

o Mentor PLHIV to carry out home based care for their members.

h. To strengthen coherent system improvements that promote linkages between health facilities and faith- and community based organisations

o Expand access to services that are not obtainable in the health facility setting, such as income generating activities, food and nutrition, spiritual care, etc.

o Strengthen linkages between community pharmacies and health facilities to support continuum of care for PLHIV.

o Promote anti-stigma campaigns to facilitate linkage of end users to community and faith- based programmes. 


\subsubsection{Health care organisation}

i. To strengthen relationships between the leadership of support group of PLHIV and the health facility management

o Improve communication/dialogue between health facility management and leadership of PLHIV support group, to improve bonding and rapport.

o Improve collaboration and participation between the leaderships of the health facility and support group, to unify decisions regarding the welfare of end users attending ART.

o Health facility leadership should understand that end users are the purpose for their existence in the facility, and do everything humanly possible to meet their needs.

o Support group leadership should mediate between end users and health facility leadership, and monitor implementation of any action points reached at all meetings held with health facility management.

\subsubsection{THEME 2: Guidelines to strengthen successes perceived by end users in ART}

\subsubsection{Delivery system design}

a. To ensure that integrated space for the provision of ART meets aesthetic and environmental standards

o ART clinic should be spacious, clean and attractive.

o Available space should be well ventilated (adequate cross ventilation) to enhance infection prevention and control.

o Space should meet both aesthetic and environmental standards for the care of chronic illnesses.

o Maintain adequate aesthetics to give the end user a homelike feeling.

o Space should have adequate basic infrastructural amenities such as clean water and uninterrupted electric power supply. 
o Health care setting should be comfortable and adequate for end users to feel relaxed.

b. To ensure uninterrupted access to ART at all times

o Understand that ART is a lifesaving treatment for HIVIAIDS; therefore, PLHIV must not experience interruptions.

o ART interruptions arising from failures in the health system should never be tolerated. Therefore, ensure that the laboratory infrastructure is functional and procurement and supply chain management of health commodities is effective at all times.

o Health care providers must commit to providing services irrespective of labour strike actions, violence, or any other form of potential interruptions.

o Where normal provision of ART becomes unattainable in its known venue, alternative measures/venue should be urgently created, such as moving ARV refills to a faith based or private health facility, where interruption may not be evident.

o Where all avenues to forestall interruption in services persist, as in famine, civil war, protracted strike actions, etc, end users should be given advance notice of such interruption and given enough drugs to cover longer period of time and/or temporarily transferred to locations where interruption is not feasible.

c. To ensure that end users have access to full medical examination/assessment at all times

o Health care providers must ensure that whenever end users turn up to the health facility, they have access to at least all the basic health assessment, such as emerging health complaints (history taking) and physical examination, including measurement of vital signs, blood pressure and body weight.

o Document all measurement results and monitor trends over time.

o Listen carefully to end users' complaints, and create a conducive environment (be fair spoken, smiling) for them to express themselves better. 
o Conduct adherence counselling and general health counselling on emerging clinical manifestations that needs medical attention.

o Open and transparent communication should be held with end users, giving them opportunities to participate in decisions regarding their care process.

o Health care providers should follow up with end users by phone or visit within two weeks of initiating therapy to assess tolerance and adherence to the antiretroviral regimen. Adherence should be reinforced at regular intervals during the course of therapy.

o Consider end users' appearance in the health facility as a very rare opportunity which must never be missed.

o For all end users who need special tests in several departments (such as $x$ ray, laboratory tests, operating room, etc), a volunteer expert end user or a nurse should be assigned to guide the end user in following all steps required to compile the test results.

\subsubsection{Self-management support}

d. To understand that end users are capable of making decisions regarding their care, and can actually carry out some basic care themselves, as well as care for their fellow end users

o Support clinical teamwork in collaboration with the end user.

o Provide end users with opportunities for on-going collaborative decisionmaking regarding their care.

o Conduct self-management assessment and empower end users to carry out self-management.

o Conduct ART/treatment education and mentoring to equip end users with knowledge and skills for self-management.

o Provide self-management resources and tools (including guidelines for selfcare).

o Provide on-going psychosocial support and encouragement, to motivate end users to continue to take charge of their care.

o Volunteers should be motivated through capacity building to empower them to be self sufficient and also support their peers. 
o Programmes should provide facilitation and logistics support to PLHIV support groups to enhance their performance.

o Programme managers/health facility authorities should facilitate support group's access to community gate keepers, to access support for their group members.

\subsubsection{Decision support}

e. To strengthen linkages and networks for expert consultation with other health facilities

o Understand that no single health facility is equipped to provide all arrays of services without dependence on another facility. Therefore, health care providers should identify possibilities of networking with other facilities and strengthen linkages with available networks for both basic and specialised health services.

o Where a strengthened network involves laboratories, health care providers must trust the capabilities of their sister facilities and accept diagnostic results issued from such other health facilities.

o Networks should also include opportunities for expert consultations, especially, where the source/primary health facility lacks capacity and resources to complete the continuum of care all alone.

o Programme managers should make provision for back-up laboratory equipment to avoid interruptions owing to equipment breakdown.

\subsubsection{Clinical information system}

f. To invest in standardising processes that ensure end users spend the least amount of time seeking health care in the facility

o Target the pharmacy and medical records units/departments for intensive process improvement measures, due to usual long waiting time in these work stations.

o Substantially involve end users in developing process improvement initiatives that are aimed at improving efficiency and effectiveness. 
o Where practicable, automation of processes such as medical records is encouraged, to speed up processes and reduce waiting time.

o Where practicable, health facilities should invest in computerised end user records, with special software to organise data and alert members of the care team to end users' needs.

o The health care providers can measure their performance against quality benchmarks set out in the software.

o Through management by facts/evidence end users are healthier, health care providers are cheered and motivated by evidence of a job well done, and the health care system saves money.

\subsubsection{Community resources}

g. To strengthen partnerships between the government, communities, civil society groups and individuals

o Build alliances and partnerships to fight stigma, and create a stigma free environment, necessary for the quality care of ART.

o Advocate to government and media organisations to support nationwide campaigns against stigma and discrimination against PLHIV.

o Government should support mass media campaigns addressing stigma reduction; while civil societies and health facilities should intensify on targeted stigma reduction programmes.

o Health care workers should be trained and sensitised around professional ethics, issues of confidentiality and stigma reduction.

o Programme managers should consider and ensure end users' audio-visual privacy when designing or allocating points of service in health facilities.

h. Support a healthy environment where PLHIV socialise with their peers and collaborate to help each other as part of the continuum of care process

o Encourage end users to join the support group of PLHIV.

o Build the capacity of PLHIV on self-management skills, and encourage them to take care of their colleagues in the support group. 
o Encourage home visits, treatment support and follow up care among end users.

o Encourage formation of smaller sub-groups of 4-8 PLHIV each, to serve as self-care quality improvement collaborative and treatment supporter to each other. The group will work together to dialogue, reflect, learn and consistently develop and implement innovative approaches in self-care. They will also assist each other in carrying out activities which they could no longer do by themselves.

\subsubsection{Health care organisation}

i. To establish an end user driven quality-based culture and framework for quality improvement in the health facility

o Establish a quality framework with a unifying purpose (mission), vision and a strategy to sustain it.

o Top management/leadership of the health facility should mobilise its workforce towards the achievement of the mission, vision and goals (long and short-term) of the health facility.

o Every health care provider should recognise that the end users are their reason for existence, while they are valuable assets to the health facility.

o Health care providers should adopt total quality management/continuous quality improvement as an integrated and routine process in their day-to-day job. It should not be seen as a programme, only.

o Everything in the health facility should be treated as a process. Every process requires inputs which then become transformed to produce some output. Processes are interrelated and depend on a series of cross-functional interventions. Therefore, breakdown departmental or professional barriers, to drive cross-functional teams.

o Institute measures to prevent extortion of end users by anybody, and to protect the rights of the individual end user.

o Automate systems such as medical records systems, where feasible, to reduce end user waiting time.

o Segment and decentralise points of services, especially, medical records and pharmacy sections, to increase speedy access to end users. 
o Where feasible, gainfully engage support group volunteers to fill human resource gaps in the health facility.

o Programme managers should ensure uninterrupted supplies of health commodities, including nutritional supplements, drugs, and where feasible, link end users to food sources.

o Support the integration of HIVIAIDS into general health care services to, improve efficiency and effectiveness in care, reduce stigma and re-inforce end user rapport with health care workers.

j. To secure commitment of top leadership to continuously improve quality and processes to meet the changing requirement and expectations of end users

o Top management should set up teams and encourage providers to work in teams. TQM relies on the use of teams to solve problems.

o Top management should continuously emphasise relentless pursuit of excellence in everything done in the health facility.

o Top management should discourage waste of resources and duplication of efforts or rework of procedures that could have been done right first time.

o Top management should encourage the use of statistical thinking to manage processes.

\subsubsection{THEME 3: Guidelines to address ART services that failed to meet end users' requirement and expectations}

\subsubsection{Delivery system design}

a. To reduce end user overload and make the ART clinic attractive to end users

o Reduce frequency of health facility visits by stable end users, through dispensing ARVs that will last 2-3 months (in line with the national ART guideline) per visit.

o Additionally, refer stable end users to lower level health facilities (primary health care) with capabilities to do follow up and ARV refills.

o Beautify the ART service area with locally available and attractive flowers, models, paintings and colours, to meet the aesthetic needs of the end users. 
b. To maintain adequate space and infrastructure that supports quality ART care

o Conduct failure mode analysis on existing infrastructure to identify early warning signs of failure.

- Develop a planned preventive maintenance schedule and commit resources to continuously maintain infrastructure (buildings, lab equipment, electric power source, water supply, etc).

o Upgrade infrastructure to match increases in end user load over time.

o Contribute to strengthening commodities logistics management system at the national, state and health facility levels, to ensure no stock-outs.

o Strengthen health facility laboratory systems; deploy skilled health care workers and build their capacity in process improvement and management.

o Ensure that the cost of poor quality caused by inadequate health systems is not transferred/passed on to the end user.

c. To ensure commodity security and availability of laboratory commodities and drugs for opportunistic infections

o Strengthen procurement and supply chain management of laboratory commodities (reagents and consumables), and drugs for opportunistic infections (including multivitamins), to ensure commodity availability and security at all times.

o Understand that drugs for opportunistic infections, multivitamins and laboratory consumables are vulnerable to abuse, as a larger population of end users need them.

o Establish transparent and accountable systems to prevent leakages and abuse of the commodities.

o Programme managers should provide comfortable seats enough for the available end user population in a health facility.

o Where feasible, all end users should not sit under harsh conditions (sun or rain).

o Provide adequate lighting in the clinic, for comfort and visualisation.

o Create adequate storage space for use by both health facility and end users'. 


\subsubsection{Self-management support}

d. To ensure end users are empowered and treated with courtesy

o Conduct workflow and workload analysis of the ART clinic to determine workstations requiring additional health care workers/capacity, and avoid health care worker burnout.

o Deploy, train and re-train additional health care workers in the CCM of care of chronic illnesses, to meet the increasing needs and dynamic expectations of end users attending ART.

o Re-distribute work or re-assign health care worker to reduce burnout, which is a possible cause of dissatisfaction between health care providers and end users.

o Strengthen the use of clinic diaries to schedule appointments for end users' clinic attendance, based on established results of workflow and workload analysis.

o Assign a point of contact for all end users willing to obtain information regarding their appointment, request changes to their appointment or advance complaints regarding the quality of health services in the health facility.

o Communicate the date, time, consulting room number, and name of doctor booked for each end user to see. This should be done well in advance using e-mail, text messages, phone calls, through treatment partners or through direct contact with the end user (whichever is more convenient in a given circumstance/location).

o End users should come on the date and time specified on the appointment diary.

o Share basic health care tasks that can be carried out by end users with them, to reduce burnout on health care providers while promoting self-management support.

o Analyse training needs and train health care workers on interpersonal communication and counselling. Institute measures (with sanctions performance issues) for discrimination, intimidation and or scolding of end users by health care workers. 


\subsubsection{Decision support}

e. To make health facility leadership to recognise that end users are the reasons for their existence in the health facility

o End users depend on health care providers' output; therefore, failure to meet or exceed their expectations would ultimately result in the loss of user loyalty, patronage and/or may even lead to the death of the health facility.

o They should seek and exercise adequate authority to ensure the protection of end users and ensure that end users leave the health facility healthy.

\subsubsection{Clinical information system}

f. To establish a system to continuously measure, report on, and improve the quality of ART from the perspectives of end users

o Establish a system to continuously monitor measures of timeliness (getting ART as soon as wanted); measures of poor communication at the health facility providing ART (end user's assessments of how often their provider listened carefully to them, explained things clearly, respected what they had to say, and spent enough time with them); and measures of end user safety (health care-associated infections, serious adverse effects, etc).

o Health care providers should put in place systems to monitor trends of the performance measures described above, and ensure end users emerge from the health facility healthy.

\subsubsection{Community resources}

g. To strengthen a system of complaints management to improve quality of ART

o Understand the socio-cultural values of the host community and end users' perceptions of the role of the health system.

o Bring health care providers and representatives of the community together to map out a complaints management system for the health facility. 
o Engage a team composed of end users, community stakeholders and health care providers to monitor, measure, report on, provide feedback and improve on the management of end users' complaints.

o Encourage the use of statistical thinking in the process of complaints management. It is management by evidence/facts (not opinion, guesswork or gut feel) that can reinforce trust and loyalty in the health facility, by the community.

\subsubsection{Health care organisation}

h. To improve overall ART processes and procedures in the health facility

o Establish a system to continuously measure, report on and improve health care processes and procedures, seeking substantial inputs from end users.

o Ensure efficiency and effectiveness of ART delivery systems through removal of unnecessary redundancies, wastages, duplication of effort and bottlenecks.

o Develop and train staff to use standard operating procedures (SOP) for all commonly recurring processes in the health facility.

o Constantly obtain feedback from end users to update the SOP from time to time, ensuring it constantly meets their expectations.

o Health facility leaders should focus on processes rather than people as the source of problems in the health care system.

In conclusion, the recommended best practice guidelines for the provision of quality ART should be used by all health care providers to improve and standardise end user focused care across all public health facilities. Proper implementation of the guideline will improve the overall quality of ART, increase user satisfaction with services and hopefully, reduce cost of ART to both the individual end user and the health facility at large, when the right treatment is gotten right first time.

\subsection{ANALYSIS AND VALIDATION OF THE GUIDELINES}

Rogers (2002) (in Moleki 2008:175) described analysis as an objective breakdown of statements into components. This is done to identify relationships between statements 
and relative hierarchy of ideas contained in the guidelines. Moleki (2008:175) cites Sieber and Mackintosh (2001) who report that analysis facilitates:

- $\quad$ Recognition of stated and unstated assumptions

- Identification of motives

- $\quad$ Comprehension of the interrelationships among statements

- Detection of logical fallacies

In contrast to analysis, Moleki (2008:175) elucidates that evaluation involves judgment about the value and logical structure of the guidelines. It also determines the extent to which the guidelines satisfy certain external criteria and/or standards. Moleki (2008:175) adds that evaluation of the guidelines allows the reader or evaluator to draw judgment and conclusions about its validity.

The above best practice guidelines for provision of quality ART was validated by subjecting it to external peer review, analysis and evaluation by experts in the field of ART service provision and end users experienced in attending ART. The guidelines were evaluated for clarity, simplicity, generality, rational structure and operational adequacy. Moleki (2008:175) has used these criteria for evaluation of her guidelines for facilitation of clinical accompaniment of critical care nursing students in open and distance learning programme.

\subsubsection{Clarity and relational structure}

The guidelines were validated for trustworthiness, semantic clarity and structural clarity. Twelve persons (including a PLHIV) working in the field of comprehensive HIVIAIDS were selected to serve as reviewers for the guidelines. The team of reviewers was composed of technical experts, expert end users, total quality management experts (with post-graduate degrees in total quality management) and programme management experts working in the field of public health. Prospective reviewers were discretely contacted over the phone, and face-to-face, in the workplace and requested to serve as reviewer for the end user focused best practice guidelines for ART. On the whole, all twelve persons accepted to participate in the review and validation process. Electronic copies of the guidelines and a brief description of processes followed by the researcher to develop the guidelines were e-mailed to each of the reviewers. Reviewers were 
requested to review and validate the guidelines for trustworthiness, semantic clarity and structural clarity. Reviewers were given options to provide written feedback or arrange to discuss their comments with the researcher. Each reviewer was given 2 weeks to revert with feedback. Reviewers were reminded prior to and on expiration of the 2 weeks timeline to revert with their comments. While 5 reviewers provided written feedback, 7 others opted to discuss their comments with the researcher. One-on-one sessions were then held with the 7 reviewers and their comments noted. Both the emailed comments and those obtained from the face-to-face meetings were compiled and used to update the guidelines. As suggested in Moleki (2008:177), the semantic clarity questioned the theoretical meaning of concepts, while structural clarity reflected on the interrelationship and connections between the conceptual framework and the theoretical framework used to develop the guidelines. The structural framework further looked at the links between the elements of the chronic care model and the recommended activities of the guidelines, to ensure they flow.

\subsubsection{Simplicity and operational adequacy}

The guidelines were evaluated to establish their suitability and applicability in the provision of end user focused ART in public health facilities. For them to be simple and operationally adequate, they had to first appear in a user friendly summary matrix/table, and further logically presented in a simple and clear language.

\subsubsection{Generality of the guidelines}

Moleki (2008:177) identifies generality as the breath of the scope and purpose of the guidelines. The guidelines were developed to facilitate staff providing ART to use proven, culturally and linguistically appropriate strategies and tools to enable end users understand all treatment options and to make decisions consistent with their values and preferences. 


\subsection{RECOMMENDATIONS FOR IMPLEMENTATION OF THE GUIDELINES}

\subsubsection{Qualifying statements}

When formulating guidelines for a disease as complex and fluid as HIVIAIDS, it is impossible to anticipate every scenario. It is expected that in specific situations, there will be valid exceptions to the approaches offered in these guidelines and sound reason to deviate from the recommendations provided within.

\subsubsection{Description of implementation strategy}

The HIVIAIDS Division of the Federal Ministry of Health (FMoH), Nigeria, oversees the development, publication, dissemination and implementation of clinical practice guidelines relevant to HIVIAIDS, in collaboration with other stakeholders, including PLHIV, development partners and implementation partners in Nigeria. Therefore, the researcher will sensitise a development partner to take the lead in advocating for the adaptation and adoption of this best practice guidelines for provision of end user focused ART in public health facilities in Nigeria. These guidelines address quality management of PLHIV, using the chronic care model.

\subsubsection{Implementation strategy using a self-care quality improvement model}

Studies have evidenced that when end users participate in their care, they are more likely to be satisfied with the care (Wouters et al 2008:5; Gill \& White 2009:14; Udoh et al 2011:220). Since the participation and substantial involvement of end users in their care is key in all end user satisfaction studies reviewed, as well as from the findings of this study, the researcher conceptualised a model to guide the implementation of the best practice guidelines. This process was conceptualised from a combination of ideas derived from the literature reviewed, findings of this study, real life experiences and the researcher's instincts. The researcher built upon the philosophy of action learning which was originally conceived by Reg Revans (Pedler 2011:5). Pedler (2011: xxi) elucidates Revans' epithet: "there can be no learning without action, and no (sober and deliberate) action without learning." Learning is 'cradled in the task,' and comes via reflection upon the experience of taking action (Pedler 2011: xxi). 


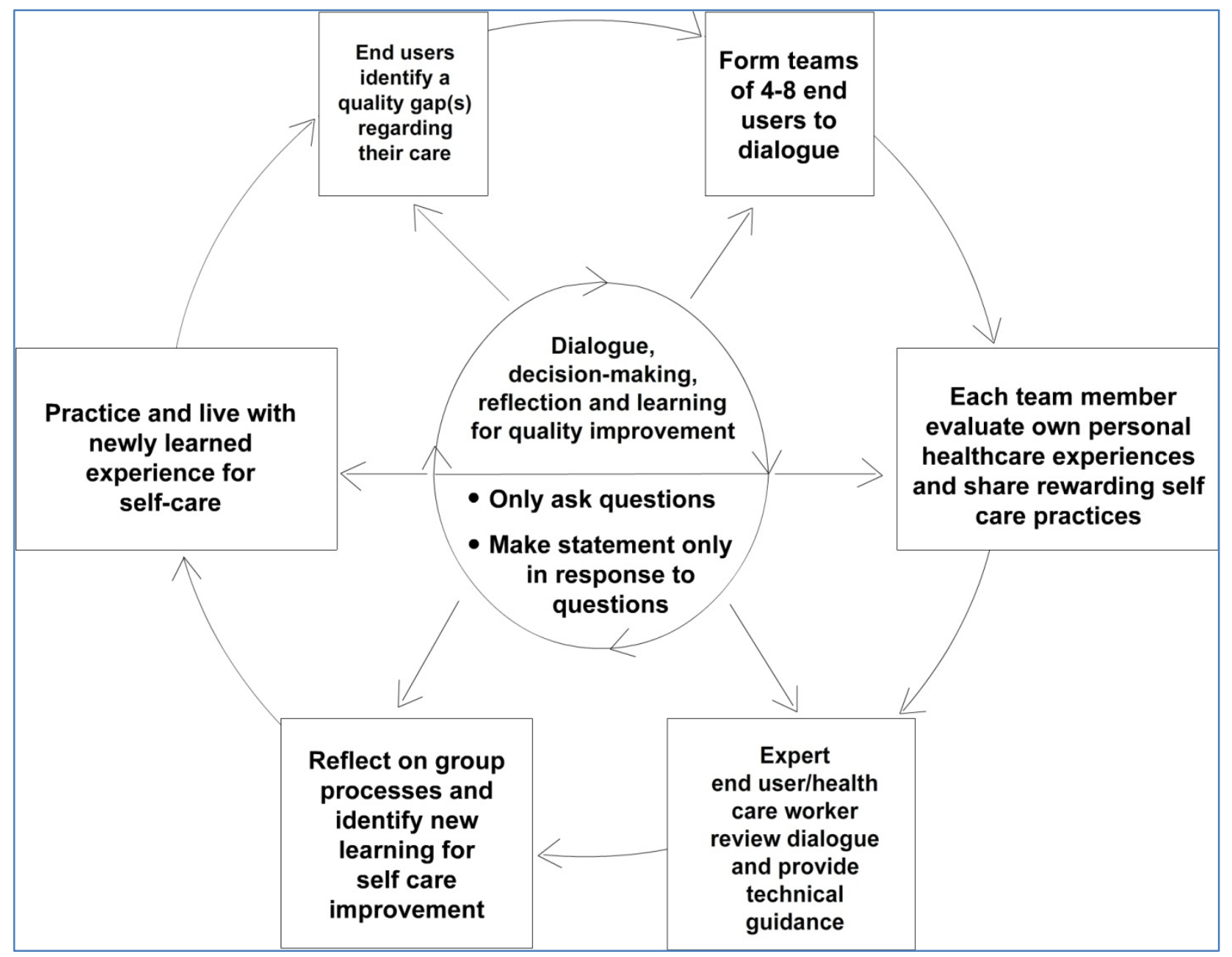

Figure 7.1 Self-care quality improvement model

This model is sugested to be used as one of the implementation tools for the best practice guidelines for ART service provision. It suggests that PLHIV should form a group of 4-8 homogenous members each, including an expert end user or a health care worker who will not be a group member, but technical advisor to the group. In each group or team, members will meet constantly (monthly or more frequently) and review/evaluate their personal health care experiences. These will include the quality of care received from the health facilities, self-care practices, and experiences with members of the community (such as stigma, discrimination, and assistance/ volunteerism). Group members will be guided by two main rules of action learning, that is, ask only questions, followed by statements. No statement shall be made if a question has not been asked. Between intervals, group members will stop the dialogue and reflect on what has been shared by every member and the process thus far. Group members identify and discuss their new learnings from the group process. The expert 
end user or health care worker set in briefly, to clarify misconceptions (if any) and provide guidance on technical aspects of the discuss, such as dosage of drugs taken, types of lab tests and their meanings, among others. Group members continue to reflect on new learnings and agree on improved requirement and expectations for ART care. Those requirements and expectations that relate to clinical care are shared with health care workers, either directly during clinic visits or through the support group leadership, as their new requirements for quality care. Others that relates to self-care are practiced out by the group members in their day-to-day living. As they practice and live with these newly learned experiences (such as brushing the teeth three times daily, eating food rich in vitamins,etc), they identify further quality issues, gaps or side effects in both functional and relational aspects, and both self-care and clinical care. The same group reconvenes as agreed upon by members and goes through the cycle all over again.

Through these cycles of action learning, end users acquire more and more knowledge and skills regarding their illness and care. Unlike what happens in the conventional support group meetings where membership is too large and personal touch impossible, the smaller and consistent group membership are able to discuss usefully, give a personal touch to each member, follow up closely (including contact tracking) with each member and learn longitudinally. The group members have opportunities to make mistakes and learn from their mistakes. They also have opportunities to reflect on their experiences, share with group members, unlearn misconceptions and set new requirements and expectations for care. The new and possibly, more sophisticated requirements are shared with health care workers as part of end users' decision-making and participation in the process of their care. This keeps health care workers on constant check and constant need to advance in knowledge and skills in order to meet or exceed the requirement and expectations of end users. Once all requirements are consistently met by health care workers, end users feel satisfied with care and are encouraged to test out and outline newer requirements. Additionally, end users are encouraged to identify, plan, do, act and study their actions, albeit in a less scientific or rigorous manner. This indeed, forms a quality improvement cycle that ultimately aims at consistently improving the quality of life of end users. 


\subsubsection{Guidelines dissemination}

Guidelines are disseminated to health care providers and end users through presentation at conferences, seminars, workshops, and pre-service trainings. Distribution methods include institutional websites, publication in journals, printed and distributed copies for those who have no access to the internet.

\subsubsection{Guidelines implementation}

The researcher will work with at least, a development partner in Nigeria, to promote the adoption of the guidelines by the HIVIAIDS division of the $\mathrm{FMoH}$. It is envisaged that the $\mathrm{FMoH}$ will mobilise a stakeholders meeting to review, analyse and critic the guidelines, with a view to adapting and adopting for implementation in public health facilities in Nigeria. Once adopted, the government of Nigeria, development partners and implementing partners will integrate the guidelines into their ART training curriculum for all cadres of health care workers. The guidelines will also be presented in continuing medical education sessions usually held in every health facility providing ART and through video and audio conferencing. Given the current drive by donors to address quality and sustainability in all their supported projects, quality indicators will be developed based on the guidelines, and a mechanism created for measurement of performance that allows stakeholders, including health care providers and end users to know to what extent specific guidelines have been implemented.

Finally, best practice booklets for end user focused ART will be developed under a separate project to facilitate the implementation of this guideline. These will contain practical solutions to common problems related to access, delivery or coordination of care, in an effort to ensure that the best practice guidelines for provision of end user focused ART are implemented and that end users receive the highest level of HIV care possible, at minimum cost to both the provider and the user.

\subsubsection{Guidelines implementation tools}

The effective implementation of these guidelines cannot be done in isolation. The following tools are necessary to support the implementation of this guideline: 
- $\quad$ National ART guidelines

- National prevention of mother to child transmission of HIV (PMTCT) guidelines

- National HIV testing and counselling guidelines

- $\quad$ Standard operating procedures for the delivery of recurring procedures in the health facility

- $\quad$ Best practice booklets for the care of people with chronic illnesses

- $\quad$ Comprehensive ART training curriculum

\subsection{CONCLUSION}

This chapter explicated the best practice guidelines for provision of end user focused quality ART in public health facilities. The guidelines were developed to facilitate staff providing ART to use proven, culturally and linguistically appropriate quality improvement strategies and tools to enable end users understand all treatment options and to make decisions consistent with their values and preferences. 


\section{List of references}

Advanced Learners' Dictionary. 2000. Sv "best practice," "end user," "guidelines," "perception". Oxford: Oxford University Press.

Agency for Health Care Research and Quality. 2010. National health care quality report. USA: AHRQ Publication (No. 11-0004).

Agha, S \& Do, M. 2009. The quality of family planning services and client satisfaction in the public and private sectors in Kenya. International Journal for Quality in Health Care, 21(2):87-96.

Agha, S, Karim, AM, Balal, A \& Solsler, S. 2007. The impact of a reproductive health franchise on client satisfaction in rural Nepal. Health Policy and Planning, 22(5):320328.

Akinola, O. 2010. Perceptions of rewards among volunteer caregivers of people living with AIDS working in faith-based organisations in South Africa: a qualitative study. Journal of the International AIDS Society, 13(1):10.

Aldana, JM, Piechulek, P \& Alsabir, A. 2001. Client satisfaction and quality of heatlh care in rural Bangladesh. Bulletin of the World Health Organization, 79:512-517.

Alemayehu, YK, Bushen, OY \& Muluneh, AT. 2009. Evaluation of HIVIAIDS clinical care quality: the case of a referral hospital in North West Ethiopia. International Journal for Quality in Health Care, 21(5):356-362.

Alemu, S, Abseno, N, Degu, G, Wondmikun, Y \& Amsalu, S. 2004. Knowledge and attitude towards voluntary counselling and testing for HIV: a community based study in northwest Ethiopia. Ethiopian Journal of Health and Development, 18(2):82-89.

Ananworanich, J, Pumpradit, W, Apateerapong, W, Siangphoe, U, Saenawat, S, Phanuphak, P \& Hirschel, B. 2008. Preference for CD4-guided versus continuous HAART in Thailand. AIDS Care, 20(3):327-330.

Andrade, TUD, Burini, DM, Mello, MDO, Bersacula, NDS, Saliba, RAD, Bravim, FT \& Bissoli, NS. 2009. Evaluation of the satisfaction level of patients attended by a pharmaceutical care program in a private communicatarian pharmacy in Vitoria, (ES, Brazil). Brazillian Journal of Pharmaceutical Sciences, 45(2):349-355.

Babbie, E \& Mouton, J. 2002. The practice of social research. New York: Oxford University Press.

Banks, DA \& Halasa, Y. 2005. Patient satisfaction and pain management in ministry of health hospitals in Jordan. Maryland: Abt Associates.

Barbosa, MF. 2011. Cancer patients under palliative care and drug utilization: profile and satisfaction. Philadelphia: WB Saunders

Beach, MC, Duggan, PS \& Moore, RD. 2007. Is patients' preferred involvement in health decisions related to outcomes for patients with HIV? JGIM:1119-1124. 
Bell, JS. 2002. Narrative inquiry: more than just telling stories. TESOL Quarterly, 36(2):207-213.

Benazzi, LEB, Figueiredo, ACL \& Bassani, DG. 2010. The user's evaluation of the ophthalmologic service offered by SUS (Brazillian Unified Health System) in an urban center in the south of Brazil. Ciencia Saude Coletiva, 15(3):861-868.

Blumenthal, PD, Gaffikin, L, Deganus, S, Lewis, R, Emerson, M \& Adadevoh, S. 2007. Cervical cancer prevention: safety, acceptability, and feasibility of a single-visit approach in Accra, Ghana. American Journal of Obstetrics and Gynecology, 196(4):407 e1-8; discussion 407 e8-9.

Booysen, FLR, Van Rensburg, HCJ, Bachmann, M, Louwagie, G \& Fairall, L. 2007. The heart in HAART: Quality of life of patients enrolled in the public sector antiretroviral treatment programme in the Free State province of South Africa. Social Indicators Research, 81(2):283-329.

Burns, N \& Grove, K. 2003. The practice of nursing research: conduct, critique and utilization. $2^{\text {nd }}$ edition. Philadelphia: WB Saunders.

Cabrera-Arana, GA, Bello-Parias, LD, Jaime, Y \& Londono-Pimienta, L. 2008. Quality as perceived by people using hospitals in the Colombian health service network restructuring programme. Rev Salud Publica (Bogota), 10(4):592-604.

Castillejo Nieves, AE. 2010. Level of satisfaction of the external user on the quality of attention of infirmary in surgical center in the hospital of support. 73-73.

Castillo, L, Dougnac, A, Vincente, I, Munoz, V, \& Rojas, V. 2007. Predictors of the level of patient satisfaction in a university hospital. Rev Med Chile, 135(6):696-701.

Chavez de Paz, P, Ramos, W \& Galarza, C. 2009. Customer's satisfaction of the outpatient dermatological service of the Dos de Mayo National Hospital: December 2006. Dermatologia Peruana, 19(1):22-31.

Chi, BH, Chansa, K, Gardner, MO, Sangi-Haghpeykar, H, Goldenberg, RL, Sinkala, M, Muchimba, M \& Stringer, JSA. 2004. Perceptions toward HIV, HIV screening, and the use of antiretroviral medications: a survey of maternity-based health care providers in Zambia. International Journal of STD \& AIDS, 15:685-690.

Chiegil, RJ. 2010. Factors relating to quality of care and their influence on antiretroviral adherence. MSc (Total Quality Management and Organizational Excellence) dissertation. Sheffield Hallam University, UK. Unpublished.

Chinn, PL \& Kramer, M. 2005. Theory and nursing integrated knowledge development. $5^{\text {th }}$ edition. St Louis: Mosby.

Chow, A, Mayer, EK, Darzi, AW \& Athanasiou, T. 2009. Patient-reported outcome measures: the importance of patient satisfaction in surgery. Surgery, 146(3):435-443.

Cleary, PD \& McNeil BJ. 1988. Inquiry: a Journal of Medical Care Organization. Provision and Financing, 25(1):25-36. 
Collins, K, Nicolson, P \& Bowns, I. 2000. Patient satisfaction in telemedicine. Health Informatics Journal, 6(2):81-85.

Colvin, M, Gumede, L, Grimwade, K, Maher, D \& Wilkinson, D. 2003. Contribution of traditional healthsers to a rural tuberculosis control programme in Hlabisa, South Africa. International Journal of Tuberculosis and Lung Disease, 7(9):S86-S91.

Coulter, A. 2005. What do patients and the public want from primary care? British Medical Journal, 331(05):1199-1201.

Coulter, A. 2010. Do patients want a choice and does it work? British Medical Journal, 341(10):c4989

Creel, LC, Sass, JV \& Yinger, NV. 2002. Overview of quality of care in reproductive heatlh:definitions and measurement of quality. New Perspectives on Quality of Care, 1:1-8.

Creswell, J. 2003. Research design: qualitative, quantitative and mixed methods. Approaches. California: Sage.

Cuba-Fuentes, M, Jurado, A \& Estrella, E. 2011. Attributes of primary care and patient satisfaction degree on a primary care facility. Rev Med Hered, 22(1):4-9.

Dao, MID, Ferreira, JF, Vallier, N, Roulin, D, Hirschel, B \& Calmy, A. 2010. Health perceptions of African HIV-infected patients and their physicians. Patient Education and Counseling, 80:185-190.

Dickhoff, J, James, P \& Wiedenbach, E. 1968. Theory in practice discipline Part 1. Practice oriented research. Nursing Research, 17(5):415-435.

Ding, L, Landon, BE, Wilson, IB, Hirschhorn, LR, Marsden, PV \& Cleary, PD. 2008. The quality of care received by HIV patients without a primary provider. AIDS Care, 20(1):35-42.

Ehlers, VJ \& Chiegil, RJ. 2011. Nigerian health workers' views concerning factors influencing paediatric adherence to anti-retroviral therapy. Health SA Geseondheid 16(1), Art \#571, 9 pages, doi:10.4102/hsag.

Family Health International. 2005. Conducting a participatory situation analysis of orphans and vulnerable children affected by HIVIAIDS: guidelines and tools. Available from:

http://www.fhi.org/NR/rdonlyres/ebkv627kkethzvtb7u6geatgcd3m6v5ilur6smufou63ey7k ociufxxajkcsvfs4nvgug44zqvnvrh/ConductOVCSitAnalysisHV.pdf (accessed on 23 February 2009).

Fonseca, SMD, Gutierrez, G \& Adami, NP. 2006. Evaluation of the satisfaction level of cancer patients with the assistance received during ambulatory antineoplastic chemotherapy. Rev Bras Enferm, 59(5):656-660. 
Francois, P, Peyrin, J, Touboul, M, Labarere, J, Reverdy, T \& Vinck, D. 2003. Evaluating implementation of quality management systems in a teaching hospital's clinical departments. International Journal for Quality in Health Care, 15(1):47-55.

Free Dictionary. [S.a.]. A definition of end user.

From: http://www.thefreedictionary.com/end+user (accessed 14 January 2012).

Gaioso, VP \& Mishima, SM. 2007. User satisfaction from the perspective of acceptability in the family health scenario.Texto \& Context Enferm, 16(4):1.

Gallardo, RJ. 2010. Quality of the attention that offers to the personal of nursing according to the opinion of the user in Preventorio Callao 2009:60-60.

Gellaitry, G, Cooper, V, Davis, C, Fisher, M, Leake, H, Date, HL \& Horne, R. 2005. Patients' perception of information about HAART: impact on treatment decisions. AIDS Care, 17(3):367-376.

Gill, L \& White, L. 2009.A critical review of patient satisfaction.Leadership in Health Services, 22(1):8-19.

Ginwalla, SK, Grant, AD, Day, JH, Dlova, TW, Macintyre, S, Baggaley, R \& Churchyard GJ. 2002. Use of UNAIDS tools to evaluate HIV voluntary counselling and testing services for mineworkers in South Africa. AIDS Care - Psychological and Socio-Medical Aspects of AIDS/HIV, 14(5):707-726.

Gouveia, GC, Souza, WVD, Luna, CF, Souza-Junior, PRBD, \& Szwarcwald, CL. 2005. Health care users' satisfaction in Brazil, 2003.Cad Saude Publica, 21(1):S109S118.

Gouveia, GC, Souza, WVD, Carlos, FL, Szwarcwald, LC, Souza Juniour, BD \& Roberto, P. 2011. Health care user satistisfaction in Pernambuco, Brazil, 2005.Ciencia Saude Coletiva, 16(3):1849-1861.

Gutierrez, E, Ramos, W, Uribe, M, Ortega-Loayza, AG, Torres, C, Momntesinos, D, Leon, O \& Galarza, C. 2009.Waiting time and its relation with the user's satisfaction in the central pharmacy of a general hospital in Lima.Rev Peru med Exp Salud Publica, 26(1):61-65.

Hasin, MA, Seeluangsawat, R \& Shareef, MA. 2001. Statistical measures of customer satisfaction for health care quality assurance: a case study. International Journal of Health Care Quality Assurance, 14(1):6-13.

Health Care Improvement Project. 2008. The improvement collaborative: an approach to rapidly improve health care and scale up quality services. University Research Co.

Henning, I, Van Rensburg, K \& Smit, L. 2004. Finding your way in qualitative research. Pretoria: Van Schaik.

Hopkins, J, Loeb, S \& Fick, D. 2009. Beyond satisfaction, what service users expect of inpatient mental health care: a literature review. Journal of Psychiatric and Mental Health Nursing, 16(10):927-937. 
Hug, Z. 2006. Six-sigma implementation through competency based perspective (CBP). Journal of Change Management, 6(3):277-289.

Hutchinson, P, Do, M \& Agha, S. 2009. Client satisfaction and the quality of family planning services: A comparative analysis of public and private health facilities in Ghana, Kenya and Tanzania. Maryland: Private Sector Partnerships-One [PSP-One].

Hutchinson, PL, Do, M \& Agha, S. 2011. Measuring client satisfaction and the quality of family planning services: a comparative analysis of public and private health facilities in Tanzania, Kenya and Ghana. BMC Health Services Research, 11(1):203.

Jackson, S. 2001. Successfully implementing total quality management tools within health care: what are the key actions? International Journal of Health Care Quality Assurance, 14(4):157-163.

Joolaee, S, Jajibabaee, F, Jafar Jalal, E \& Bahrani, N. 2011. Assessment of patient satisfaction from nursing care in hospitals of Iran University of Medical Sciences. Hayat Journal of Faculty of Nursing \& Midwifery, 17(1):1.

Joseph, C \& Nichols, S. 2007. Patient satisfaction and quality of life among persons attending chronic disease clinics in South Trinidad, West Indies.West Indian Medical Journal, 56(2):108-114.

Kabbash, IA, Hassan, NM, Al-Nawasy, AN, Attalla, AA \& Mekheimer, SI. 2010. Evaluation of HIV voluntary counselling and testing services in Egypt. Part 1: client satisfaction. Eastern Mediterranean Health Journal, 16(5):481-490.

Karunamoorthi, K, Rajalakshmi, M, Babu, SM \& Yohannes, A. 2009. HIVIAIDS patient's satisfactory and their expectations with pharmacy service at specialist antiretroviral therapy (ART) units. European Review for Medical and Pharmacological Sciences, 13:331-339.

Lima, ACSD, Cabral, ED, \&Vasconcelos, MMVB. 2010. Patient satisfaction at Specialist Dental Clinics in Recife, Pernambuco State, Brazil. Cad Saude Publica, 26(5):591-1002.

Lima, MADDS, Ramos, DD, Rosa, RB, Nauderer, TM \& Davis R. 2007. Access and quality care in health care centers from the users' point of view. Acta Paul Enferm, 20(1):12-17.

Lima-Costa, MF \& Loyola Filho, Al. 2008. Associated factors to the use and the satisfaction with health services among national health system users in the metropolitan region of Belo Horizonte, State of Minas Gerais, Brazil. Epidemiol Serv Saude, 17(4):247-257.

Lincoln, YS \& Guba, EG. 1985. Naturalistic inquiry. Beverly Hills, CA: Sage.

Liu, Y \& Wang, G. 2006. Inpatient satisfaction with nursing care and factors influencing satisfaction in a teaching hospital in China. Journal of Nursing Care Quality, 22(3), 266271. 
Lofland, L \& Wilkblud, G. 2001. Facilitating and obstructing factors for development of learning in clinical practice: a student perspective. Journal of Advanced Nursing, 34(1):43-729.

Lopez-Soto, OP, Cerezo-Correa, MdP \& Paz-Delgado, AL.2010. Relationship variables with the satisfaction of patients of dental services.Rev Gerenc politicas Salud (Bogota), 9(18):124-136.

Machado, MC, Medina, FMC \& Kara-Jose, N. 2010. Perception of the users, health professionals and managers of the assistance model of the regional Ophthalmologic Hospital of Divinolandia, Sao Paulo.Arg Bras Oftalmol, 73(3):276-281.

Malta, M, Maya, LP, Clair, S, Freitas, F \& Bastos, BI. 2005. Adherence to antiretroviral therapy: a qualitative study with physicians from Rio de Janeiro, Brazil. Cadernos de Saude Publica, 21(5):1424.

Mayeye, FB, Lewis, HA \& Oguntibeju, OO. 2010. An assessment of adolescent satisfaction with reproductive primary health care services in the Eastern Cape Province, South Africa. West Indian Medical Journal, 59(3):274-279.

Mdondolo, N, De Villiers, L \& Ehlers, VJ. 2003. Cultural factors associated with the management of breast lump amongst Xhosa women. Health SA Gesondheid, 8(3):8697.

Medeiros, FA, DeAraujo-Souza, GC \& Albuquerque-Barbosa e Iris do Ceu Clara-Costa, AA. 2010. Basic health unit embracement: focusing on user satisfaction. Rev Salud Publica (Bogota), 12(3):402-413,

Mehrabi, F, Nasiripour, A \& Delgoshaei, B. 2008. Customer focus level following implementation of quality improvement model in Tehran social security hospitals. International Journal of Health Care Quality Assurance, 21(6):562-568.

Mfinanga, SG, Kahwa, A, Kimaro, G, Kilale, A, Kivuyo, S, Senkoro, M, Ngowi, B, Mtandu, R, Mutayoba, B, Ngadaya, E \& Mashoto, K. 2008. Patient's dissatisfaction with the public and private laboratory services in conducting HIV related testing in Tanzania. BMC Health Services Research, 8:167.

Miller, LG, Huffman, HB, Weidmer, BA \& Hays, RD. 2002. Patient preferences regarding antiretroviral therapy. International Journal of STD and AIDS, 13:593-601

Minkman, M, Ahaus, K \& Huijsman, R. 2007. Performance improvement based on integrated quality management models: what evidence do we have? A systematic literature review.International Journal for Quality in Health Care, 19(2):90-104.

Mishima, SM, Pereira, FH, Matumoto, S, Fortuna, CM, Pereira, MJB, Campos, AC \& Paula, VGd. 2010. Assistance in family health from the perspective of users. Rev Latino-Am Enfermagem, 18(3):436-443.

Miranda, SP \& Vargas, DD. 2009. Satisfaction of patients from a psychosocial care center for alcohol and drugs with nurse care. SMAD Rev Electronica Saude Mental Alcohol Drug, 5(2):1-15. 
Moleki, MM. 2008. Critical care nursing students' experience of clinical accompaniment in open distance learning (ODL): A phenomenological perspective. D Litt et Phil (Health Studies) thesis. University of South Africa, Pretoria.

Mooij, MD \& Hofstede, G. 2010. The Hofstede model - applications to global branding and advertising strategy and research. International Journal of Advertising, 29(1), 85110.

Morin, D, Godin, Alary, M, Sawadogo, MR, Bernier, M, Khonde N, Kintin, F, Kone, A, N'Dour, M, Pepin, J, Rached, S, Sobela, F, Soto, J, Sylla, M \& Traore, C. 2008. Satisfaction with health services for STIS, HIV, AIDS among a high-risk population in West Africa.AIDS Care - Psychological and socio-medical aspects of AIDS/HIV, 20(3):388-394.

Mouton, J.1996. Methods of social research. Juta: Cape Town.

Mshana, GH, Wamoyi, J, Busza, J, Zaba, B, Changalucha, J, Kaluvya, S \& Urassa, M. 2006. Barriers to accessing antiretroviral therapy in Kisesa, Tanzania: a qualitative study of early rural referrals to the national programme. AIDS Patient Care and STDS, 20(9):649-657.

Mulhall, A. 2002. Methodological issues in nursing research. In the field: notes on observation in qualitative research. 2003. Blackwell. From:

www.angelfire.com/.../images/UnstrucObservationpaper.pdf · (accessed on 15 February 2012).

Muller, EV \& Greco, M. 2010. Evaluation of users' satisfaction with the services of the intermunicipal health consortia at the northwest of Parana state. Ciencia Saude coletiva, 15(3):925-930.

Muza, R \& Muza, P. 2008. Patients' satisfaction with three dental specialties. Rev Chil Salud Publica, 12(1):12-17.

Myburgh, NG, Solanki, GE, Smith, MJ \& Lalloo, R. 2005. Patient satisfaction with health care providers in South Africa: the influences of race and socioeconomic status. International Journal of Quality in Health Care, 17(6):473-477.

Nakhaee, N \& Mirahmadizadeh, AR. 2005. Iranian women's perceptions of family planning services quality: a client satisfaction survey. European Journal of Contraception and Reproductive Health Care, 10(3):192-198.

Nduna, M \& Farlane, L. 2009. Women living with HIV in South Africa and their concerns about fertility. AIDS Behaviour, 13:S62-S65.

Oerman, MH. 2001. Professional nursing practice: conceptual approach. Philadelphia: Lippincott.

Oliveira, DFD, Arieta, CEL, Temporini, ER \& Kara-Jose, N. 2006. Quality of health care: patient satisfaction in a university hospital. Arq Bras Oftalmol, 69(5):731-736.

Oliveras, E, Larsen, U \& David, PH. 2005. Client satisfaction with abortion care in three Russian cities.Journal of Biosocial Sciences, 37:585-601. 
Olupot-Olupot, P, Katawera, A, Cooper, C, Small, W, Anema, A \& Mills, E. 2008. Adherence to antiretroviral therapy among a conflict-affected population in northeastern Uganda: a qualitative study. AIDS, 22(14):1882-1884.

Page-Shipp, LS, Charalambous, S, Roux, S, Dias, B, Sefuti, C, Churchyard, GJ \& Grant, AD. 2007. Attitudes to directly observed antiretroviral treatment in a workplace HIV care programme in South Africa. Sexually Transmitted Infections, 85:383-386.

Paiva, SMAD \& Gomes, ELR. 2007. Hospital care: assessment of users' satisfaction during hospital stay. Rev Latino-Am Enfermagem, 15(5):973-979.

Patton, MQ. 2002. Qualitative research and evaluation methods. $3^{\text {rd }}$ edition. California: Thousand Oaks.

Pedler, M. 2011. Action learning in practice. $4^{\text {th }}$ edition. Burlington: Gower publishing company.

Phaladze, NA, Human, S, Dlamini, SB, Hulela, SB, Hadebe, IM, Sukati, NA, Makoae LN, Seboni, NM, Moleko, M \& Holzemer, WL. 2005. Quality of life and the concept of "living well" with HIVIAIDS in sub-Saharan Africa. Journal of Nursing Scholarship, 37(2):120-126.

Pietrovsk, V \& Dall'Agnol, CM. 2006. Significant situations in the space and context of hemodialysis: what do users say about? Rev Bras Enferm, 59(5):630-635.

Polit, DF \& Beck, CT. 2004. Nursing research, principles and methods.Philadelphia: JB Lippincott.

Pompeo, DA, Pinto, MH, Cesarino, CB, Araujo, RRDFD \& Poletti, NAA. 2007. Nurses performance on hospital discharge: patients' point of view. Acta Paul Enferm, 20(3):345350.

Pope, C, Ziebland, S \& Mays, N. 2000. Qualitative research in health care: analysing qualitative data. British Medical Journal, 320(7227):114-116.

Porzsolt, F, Ackermann, M \& Amelung, V. 2007. The value of health care - a matter of discussion in Germany. BMC Health Services Research, 7(1):1-8.

Priebe, SA, Matanov, A, Demi, N, Simic, JB, Jovanovic, S, Gajic, M, Radonic, E, Bajraktarov, S, Boderscova, L, Konatar, M, Nica, R \& Muijen, M. 2011. Community mental health centers initiated by the South-Eastern Europe stability pact: evaluation in seven countries. Community Mental Health Journal, DOI 10.1007/s10597-011-9417-6.

Proctor, A, Jenkins, TR, Loeb, T, Elliot, M \& Ryan, A. 2006. Patient satisfaction with 3 methods of postpartum contraceptive counselling: a randomized, prospective trial. Journal of Reproductive Medicine, 51(5):377-382.

Rad, M, Haghani, J, Shahravan, A \& Khosravifar, A. 2009. Qualitative assessment of the dental health services provided at a dental school in Kerman, Iran. Braz Oral Res, 23(4):377-380. 
Ramchandani, SR, Mehta, SH, Saple, DG, Vaidya, SB, Pandy, VP, Vandrevu, R, Rajasekaran, S, Bhatia, V, Chowdhary, A, Bollinger, RC \& Gupta, A. 2007.Knowledge, attitudes, and practices of antiretroviral therapy among HIV-infected adults attending private and public clinics in India.AIDS Patient Care and STDs, 21(2):129-142.

Rao, D, Kekwaletswe, TC, Hosek, S, Martinez, J \& Rodriguez, F. 2007. Stigma and social barriers to medication adherence with urban youth living with HIV.AIDS Care, 19(1):28-33.

Regmi, K \& Madison, J. 2010. Ensuring patient satisfaction with second-trimester abortion in resource-poor settings.International Journal of Gynecology and Obstetrics, 108(1):44-47.

Ricci, NA, Wanderley, FDS, Oliveira, MS \& Rebellato, JB. 2011. The Sao Carlos Hospital School: assessment of its functioning by user satisfaction. Ciencia \& Saude Coletiva, 16(1):1125-1134.

Roura, M, Urassa, M, Busza, J, Mbata, D, Wringe, A \& Zaba, B. 2009. Scaling up stigma? The effects of antiretroviral roll-out on stigma and HIV testing.Early evidence from rural Tanzania.Sexually Transmitted Infections, 85:308-312.

Santos, FRD, Lima, SA, Elias, FCDA, Magacho, E, Oliveira, LAD, Fernandes, N, Carmo, DWB, Abrita, RR \& Bastos, MG. 2008. Patient's satisfaction with interdisciplinary care in a clinic of prevention of chronic kidney disease.J Bras nefrol, 30(2):151-156.

Schumaker, LL \& Bond, VA. 2008. Antiretroviral therapy in Zambia: colours, 'spoiling,' 'talk' and the meaning of antiretrovirals. Social Science \& Medicine, 67:2126-2134.

Senarath, U, Fernando, DN \& Rodrigo, I. 2006. Factors determining client satisfaction with hospital-based perinatal care in Sri Lanka.Tropical Medicine and International Health, 11(9):1442-1451.

Shaikh, BT. 2005. Quality of health care: an absolute necessity for patient satisfaction. Journal of the Pakistan Medical Association, 55(1):515-516.

Silva, RMD, Beck, CLC, Lopes, LFD, Magnago, TSBDS, Prestes, FC \& Tavares, JP. 2010. Patient satisfaction in the postoperative of fracture as nursing care: descriptive study. Online Brazillian Journal of Nursing, 9(2):1.

Singh, D \& Ham, C. 2006. Improving care for people with long-term conditions: a review of UK and international frameworks. Birmingham: HSMC, University of Birmingham \& NHS Institute for Innovation and Improvement.

Sisson, MC, Oliveira, MCd, Conill, EM, Pires, D, Boing, AF \& Fertonani, HP. 2011. Users' satisfaction with the use of public and private health services within therapeutic itineraries in southern Brazil. Interface Comunic Saude Educ, 15(36):123-136.

Sovd, T, Mmari, K, Lipovsek, V \& Manaseki-Holland, S. 2006. Acceptability as a key determinant of client satisfaction: lessons from an evaluation of adolescent friendly health services in Mongolia. Journal of Adolescent Health, 38(5):519-526. 
Stanhope, L \& Lancaster, K. 2006. Community public health. St Louis: Mosby.

Stenson, AL, Charalambous, S, Dwadwa, T, Pemba, L, Toit, DD, Baggaley, R, Grant, AD \& Churchyard, GJ. 2005. Evaluation of antiretroviral therapy (ART)-related counselling in a workplace - based ART implementation programme, South Africa. AIDS Care, 17(8):949-957.

Stommel, M \& Wills, CE. 2004. Clinical research: concepts and principles for advanced practice nurses.Philadelphia: Lippincott Williams \& Wilkins.

Tindyebwa, D, Kayita, J, Musoke, P, Eley, B, Nduati, R, Coovadia, H, Bobart, R, MboriNgacha, D \& Kieffer, PM. (eds). 2005. Handbook on paediatric AIDS in Africa. Uganda: ANECCA.

Tobi, P, George, G, Schmidt, E \& Renton, A. 2008. Antiretroviral treatment and the health workforce in South Africa: how have ART workers been affected by scaling up? Tropical Medicine and International Health, 13(12):1452-1458.

Torpey, KE, Kabaso, ME, Mutale, LN, Kamanga, MK, Mwango, AJ, Simpungwe, J, Suzuki, C \& Mukadi, YD. 2008. Adherence support workers: a way to address human resource constraints in antiretroviral treatment programmes in the public health setting in Zambia. Plos One, 3(5):1-9.

Udoh, BE, Eze, JC \& Okeji, MC. 2011. Comparative assessment of patient satisfaction with obstetric sonography between missionary and government hospitals in southeastern Nigeria. Journal of Diagnostic Medical Sonography, 27(5):220-224.

Wagner, G, Ryan, G \& Taylor, S. 2007. Formative evaluation of antiretroviral therapy scale-up efficiency in sub-Saharan Africa. AIDS Patient Care and STDs, 21(11):871887.

Wakefield, DS, Ward, MM \& Wakefield, BJ. 2007. A 10-right framework for patient care quality and safety. American Journal of Medical Quality, 22(2):103-111.

Walker, LD \& Avant, KC. 2004. Strategies for theory construction in nursing. $4^{\text {th }}$ edition. California: Pearson Prentice Hall.

Wielawski, IM. 2006. Improving chronic illness care. Birmingham: HSMC, University of Birmingham \& NHS Institute for Innovation and Improvement

Wikipedia. [S.a.]. A definition of reflexivity.

From: http://en.wikipedia.org/wiki/Reflexivity (social theory) (accessed 14 January 2012).

Wong, R \& Bradley, EH. 2009. Developing patient registration and medical records management system in Ethiopia. International Journal of Quality in Health Care, 21(4):253-258.

World Health Organization. 2004. Standards for quality HIV care: a tool for quality assessment, improvement, and accreditation. Report of a WHO Consultation Meeting on the Accreditation of Health Service Facilities for HIV care, 10-11 May 2004, Geneva, Switzerland. 
World Health Organization. 2006. Antiretroviral therapy for HIV infection in adults and adolescents: recommendations for a public health approach.

Available from: http://www.who.int/hiv/pub/guidelines/artadultguidelines.pdf (accessed on 18 September 2011).

Wouters, W, Heunis, C, Van Rensburg, D \& Meulemans, H. 2008. Patient satisfaction with antiretroviral services at primary heatlh care facilities in the Free State, South Africa - a two-year study using four waves of cross-sectional data. BMC Health Services Research, 8(210):1-10.

Yakong, NY, Rush, KL, Bassett-Smith, J, Bottorff JL \& Robison C. 2010. Women's experiences of seeking reproductive health care in rural Ghana: challenges for maternal health service utilization. Journal of Advanced Nursing, 66(11):2431-1241.

Zanetti, ML, Otero, LM, Biaggi, MV, Santos, MAd, Peress, DS \& Guimaraes, FPdM. 2007. Satisfaction of diabetes patients under follow-up in a diabetes education program.Rev Latino-Am Enfermagem, 15(4):583-589.

Zungu, LI, Abu, UO, Ogunbanjo, GO \& Setswe, GK. 2009. Utilisation of the national antiretroviral therapy guidelines among health care professionals working in Abuja treatment centres, Nigeria. Africa Journal of Primary Health Care and Family, 7(3):1-10. 
Annexure A

Certificate of participation in the training on research ethics 


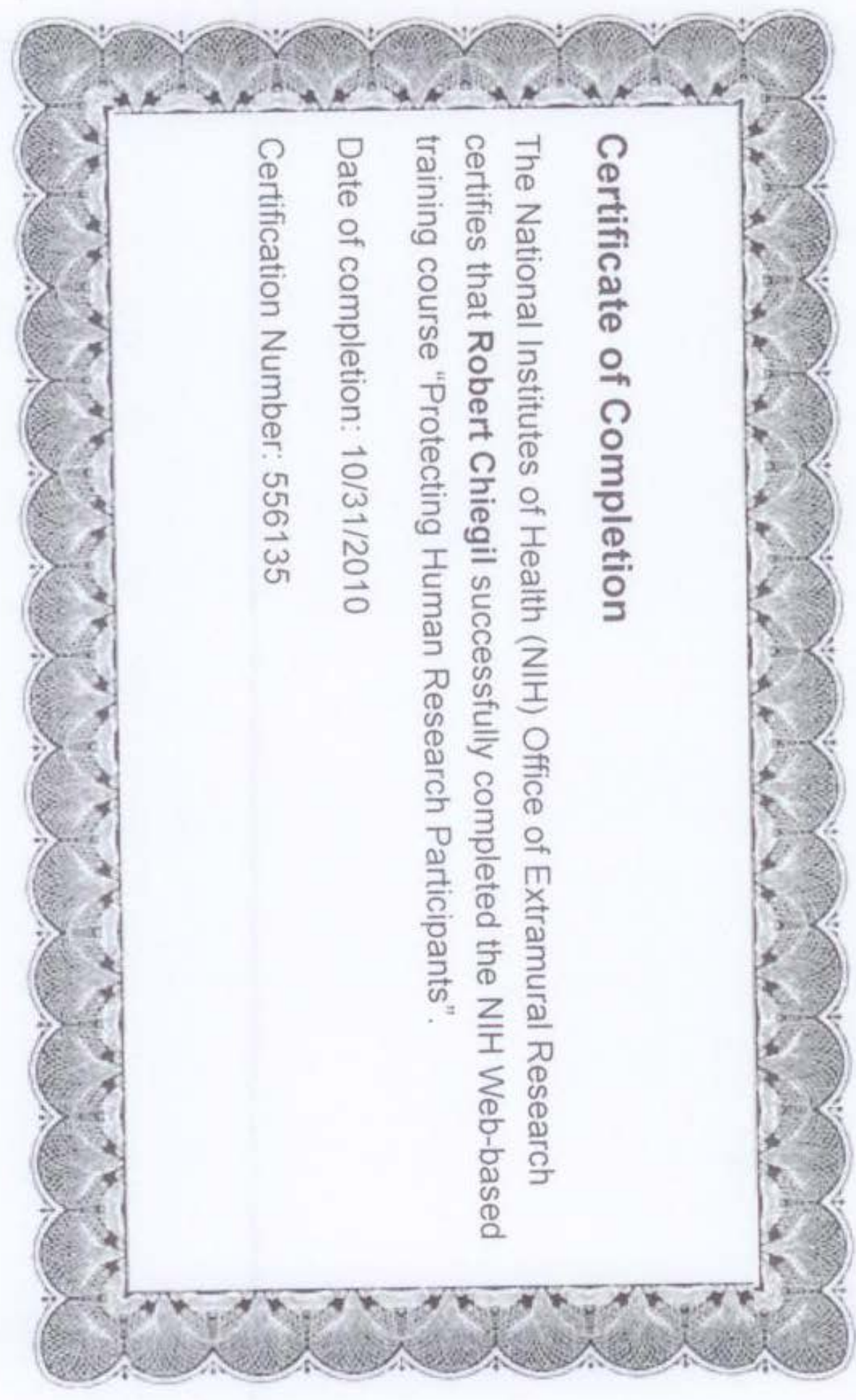




\section{Annexure B}

Requests for permission to conduct the study 
FHI/GHAIN, Godab Plaza, Plot 1073-A1

JS Tarka Street, Area 3, Garki

Abuja

October 31, 2010

The Chairperson

National Health Research Ethics Committee

Federal Ministry of Health

Abuja

Sir,

\section{Application for Ethical Clearance}

I write to apply for ethical clearance to conduct a study entitled Nigerian health service users' views concerning quality of antiretroviral therapy in public health facilities. The purpose of the study is to explore and describe the requirements and expectations of ART service users regarding quality of services received in public health facilities in Nigeria. Data will be collected through focus group interviews with people living with HIV/AIDS attending ART services in public health facilities.

I am a predoctoral student of the University of South Africa, Pretoria, working under research supervision of Prof. Lindiwe I. Zungu [+27 (0) 12-4292058, zunguli@unisa.ac.za ] and Prof. T. Mhlongo (Joint Supervisor).

Attached herewith for your perusal are:

1. Filled Application form for ethical clearance

2. Protocol/proposal summary sheet

3. Informed verbal consent form

4. Researcher's Biographical sketch

5. Certificate of completion of NIH web-based ethics training course

6. Research proposal

I look forward to hearing from you, please.

Yours truly,

Robert J. Chiegil

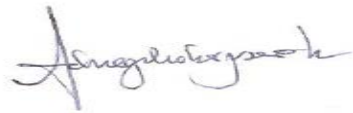

Student Researcher

08035615573

rchiegil@ghain.org 


\section{NATIONAL HEALTH RESEARCH ETHICS COMMITTEE}

APPLICATION FOR ETHICAL APPROVAL

Title of Research Project...Nigerian health service users' views concerning quality of antiretroviral therapy in public health

facilities

Principal Investigator's Name: Robert Joseph Chiegil...........

Department...Health Studies...Faculty College of Human Sciences, University of South Africa

Cell Phone No...08035615573.................Extension............ E-mail...rchiegil@ghain.org

Area of Research (e.g. Malaria, HIV/AIDS, non-communicable disease)...HIV/AIDS.........

Research includes the following elements (tick as appropriate):

\section{Nature of Study}

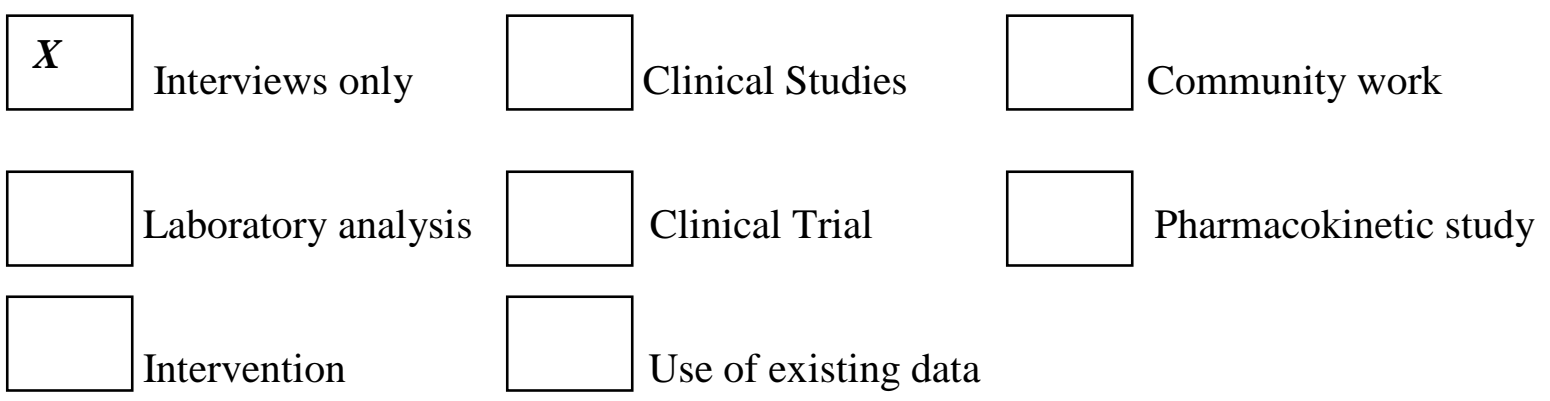

Others, please specify:...Focus Group Discussions....

2. Type of the Project
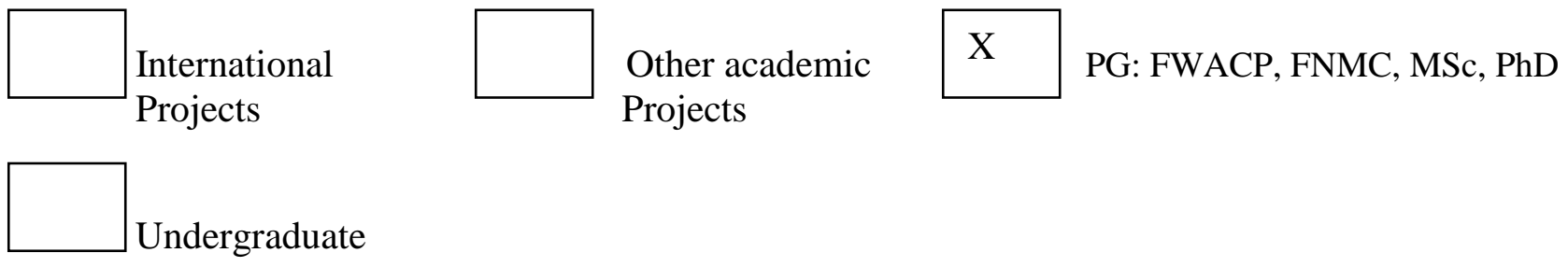

\section{Mode of Funding}

\begin{tabular}{|l|l}
\hline $\mathrm{X}$ & Self Funded \\
\hline
\end{tabular}

Nationally/Institutionally

Funded
Internationally

Funded 


\section{Study Requirements}

Biological Sampling required?

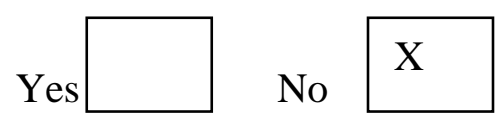

If yes, which samples?

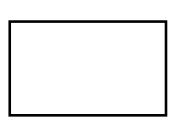

Blood

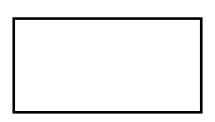

Saliva

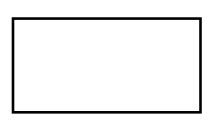

Urine

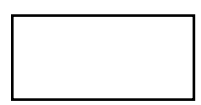

Tissue

Biopsy

Others (Please Specify).

\section{Disposition of samples}

Are samples going to be shipped out of Nigeria?

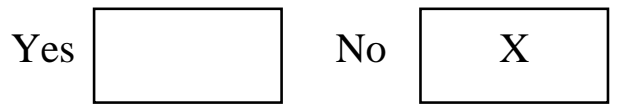

\section{Duration of Study 6 mos}

Corresponding Investigator's Name: ...Robert J. Chiegil.

Supervisor's Name (where applicable) ...Prof Lindiwe Zungu..............

Contact Address:... Department of Health Studies, University of South Africa, PO Box 392, UNISA.

Phone:...+27 (0) 12-429

2058

Fax:...+27 (0) 12-429 6688... E-mail:... zunguli@unisa.ac.za

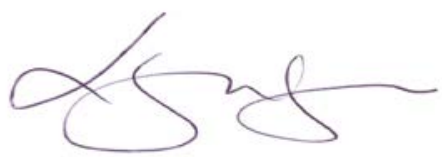

Signature:

Date: 17 October 2010 


\section{DECLARATION IN SUPPORT OF APPLICATION FOR ETHICAL APPROVAL}

I certify that the information provided in the study protocol is true to the best of my knowledge. I agree to undertake the research according to the ethical principles described in National Code for Health Research Ethics, relevant Federal and local laws, ICH-GCP6 guidelines (where applicable), government and institutional guidelines and regulations. I understand that the Ethics Committee will provide Ethical Approval for this protocol for a specified time period not exceeding 1 year and that continuation of the research beyond this period will require renewal of the approval. I understand that the Ethics Committee may, without prior notice, observe of cause to be observed, the research for which approval has been given in order to ensure compliance with approved protocol, suspend or terminate the conduct of this research if necessary.

Name of Investigator: ....Robert Joseph Chiegil..........

Signature.........

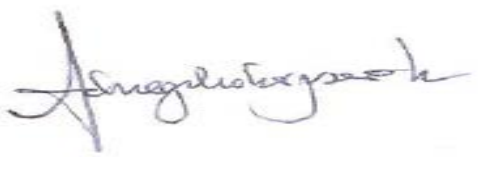

Date: ...6 October 2010 


\section{Annexure C}

Letter granting permission to conduct the study 
National Health Research Ethics Committee of Nigeria (NHREC)

Promoting Highest Ethical and Scientific Stan dards for Health Research in Nigeria

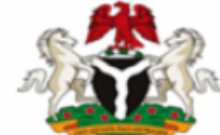

Federal Ministry of Health

NHREC Protocol Number NHREC/01/01/2007-16/03/2011

NHREC Approval Number NHREC/01/01/2007-12/05/2011

Date: May 12, 2011

$\underline{\text { Re: Nigerian Health Service Users' Views Concerning Quality Of Antiretroviral Therapy }}$ In Public Health Facilities

Health Research Ethics Committee (HREC) assigned number: NHREC/01/01/2007

Name of Principal Investigator:

Robert Chiegil

Address of Principal Investigator:

Family Health International, GHAIN

Plot 1073, J.S. Tarka Street, Godab Plaza, Area 3, Garki,

Abuja ,

T: 234.94615555 ext. 503 | M: 080.356 .15573

| rchiegil@ghain.org | www.fhi.org

Date of receipt of valid application: 16-03-2011

Date when final determination of research was made: 12-05-2011

Notice of Full Committee Approval

This is to inform you that the research described in the submitted protocol, the consent forms, advertisements and other participant information materials have been reviewed and given full committee approval by the National Health Research Ethics Committee.

This approval dates from $12 / 05 / 2011$ to $11 / 05 / 2012$. If there is delay in starting the research, please inform the HREC so that the dates of approval can be adjusted accordingly. Note that no participant accrual or activity related to this research may be conducted outside of these dates. All informed consent forms used in this study must carry the HREC assigned number and duration of HREC approval of the study. In multiyear research, endeavor to submit your annual report to the HREC early in order to obtain renewal of your approval and avoid disruption of your research.

The National Code for Health Research Ethics requires you to comply with all institutional guidelines, rules and regulations and with the tenets of the Code including ensuring that all adverse events are reported promptly to the HREC. No changes are permitted in the research without prior approval by the HREC except in circumstances outlined in the Code. The HREC reserves the right to conduct compliance visit your research site without previous notification.

Signed

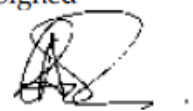

Clement Adebamowo BMChB Hons (Jos), FWACS, FACS, DSc (Harvard)

Honorary Consultant Surgeon, Director, West African Center for Bioethics and

Chairman, National Health Research Ethics Committee of Nigeria (NHREC)

Tel: +234-09-523-8667

E-mail. chairman@nhrec.net, secretary@nhrec.net. deskofficer@nhrec.net.

URL: http / Mwww nhrec net. 
Annexure D

Ethical clearance certificate from Unisa 


\section{UNISA}

\section{UNI VERSITY OF SOUTH AFRI CA Health Studies Research \& Ethics Committee (HSREC) Faculty of Human Sciences CLEARANCE CERTI FI CATE}

Project Title: Nigerian health service users' views concerning the quality of antiretroviral therapy in public health facilities.

Researcher: Robert Joseph Chiegil

Supervisor/Promoter: Prof LI Zungu

Joint Supervisor/Joint Promoter: N/A

Department: Health Studies

Degree: $\quad$ DLITT ET PHIL (Health Studies)

\section{DECISION OF COMMI TTEE}

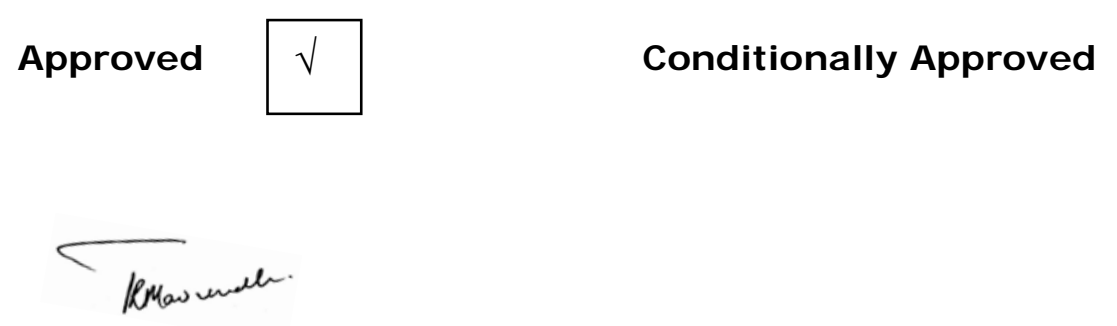

Prof TR Mavundla RESEARCH COORDI NATOR

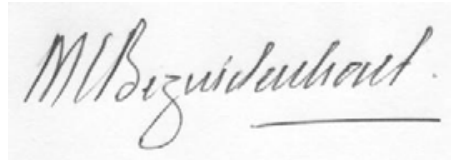

Prof MC Bezuidenhout ACADEMI C CHAI RPERSON: DEPARTMENT OF HEALTH STUDIES PLEASE QUOTE THE PROJECT NUMBER IN ALL ENQUIRES 


\section{Annexure E}

\section{Consent letter for FGD participants}




\section{Consent Letter}

IRB Research approval number: NHREC/01/01/2007

This approval will elapse on: 11/05/2012

Title of the research: Nigeria health service users' views concerning the quality of antiretroviral therapy in public health facilities.

Name(s) and affiliation(s) of researcher(s) of applicant(s): This study is being conducted by Robert Joseph Chiegil, a doctoral student of the University of South Africa, Pretoria

Sponsor(s) of research: This study is self-sponsored by the student, Robert J. Chiegil

Purpose(s) of research: The purpose of this study is to explore and describe the requirements and expectations of ART service users regarding quality of services received in public health facilities in Nigeria. The study seeks to answer the broad question: "What are the requirements and expectations of ART service users regarding services received in public health facilities?”

\section{Procedure of the research, what shall be required of each participant and approximate total number of participants that would be involved in the research:}

Today, I would like to request that you participate in a focus group discussion, which should take approximately one hour. I will be asking you questions about your experiences and views regarding quality of antiretroviral care received in this health facility. I would like to ask for your consent to audio record this interview. The purpose of the audio record is to ensure I have complete documentation of the FGD and to assist me in my analysis and write up. Your participation is completely voluntary. If you do not wish to participate, you may individually or collectively stop the FGD at any time. Your refusal to participate, to answer some questions or to withdraw during the course of the FGD will not involve any penalty, affect your ability to receiving treatment in the health 
facility or affect you in any other way. You are expected to speak freely to the issues I'll be bringing forward, except you do not wish to speak to any particular issue. Your responses are completely anonymous, I will not record your name in any of my transcripts or notes and your name will not appear anywhere in the final write up. The original responses that I gather from the interview will only be available to me. In total I expect to discuss with 80 participants, representing 6 focus groups throughout the country.

\section{Expected duration of research and of participant(s)' involvement:}

In total, your participation in this FGD will last no more than an hour, and that will be all about your involvement.

Risk(s): I do not foresee any risk to you as a result of your participation in this study, or any time following this study.

Costs to the participants, if any, of joining the research: Your participation in this research will not cost you anything.

Benefit(s): The purpose of this study is to explore your views, requirements and expectations regarding quality of ART services you receive in public health facilities in Nigeria. The information you provide will therefore be used to inform policy changes and development of programmes to meet your requirement and expectations.

Confidentiality: All information collected in this study will be analysed and no name will be recorded. No part of the final report can be linked to you in anyway and your name or any identifier will not be used in any publication or reports from this study. As part of our responsibility to conduct this research properly, officials from NHREC may have access to these records.

Voluntariness: Your participation in this research is entirely voluntary. 
Alternatives to participation: If you choose not to participate, this will not affect your treatment in this hospital in any way.

Due inducement(s): You will be compensated for lost wages; cost of transport to and from the research site but you will not be paid any fees for participating in this research.)

Consequences of participants' decision to withdraw from research and procedure for orderly termination of participation: You can also choose to withdraw from the research at anytime. Please note that some of the information that has been obtained about you before you chose to withdraw may have been modified or used in reports and publications. These cannot be removed anymore. However the researcher promise to make good faith effort to comply with your wishes as much as is practicable.

Modality of providing treatments and action(s) to be taken in case of injury or adverse event(s): Should there be any injury as a result of your participation in this research, you will be treated at any public health facility within your locality and the research will bear the cost of this treatment.

What happens to research participants and communities when the research is over: The researcher will inform you of the outcome of the research through your Support Group meetings.

Statement about sharing of benefits among researchers and whether this includes or exclude research participants: If this research leads to commercial services, the University of South Africa and Robert J. Chiegil shall jointly own it. There is no plan to contact any participant now or in future about such commercial benefits.

Any apparent or potential conflict of interest: The researcher is a staff of one of the implementing partners providing technical assistance to the Government of Nigeria in the implementation of comprehensive ART services. He is however, not aware of any information that may cause him not to do the work with fear or favour. 


\section{Statement of person obtaining informed consent:}

I have fully explained this research to and have given sufficient information, including about risks and benefits, to make an informed decision.

DATE: SIGNATURE: NAME:

\section{Statement of person giving consent:}

I have read the description of the research or have had it translated into language I understand. I have also talked it over with the researcher to my satisfaction. I understand that my participation is voluntary. I know enough about the purpose, methods, risks and benefits of the research study to judge that I want to take part in it. I understand that I may freely stop being part of this study at any time. I have received a copy of this consent form and additional information sheet to keep for myself.

DATE: SIGNATURE:

NAME:

WITNESS' SIGNATURE (if applicable):

WITNESS’ NAME (if applicable):

Detailed contact information including contact address, telephone, fax, e-mail and any other contact information of researcher(s), institutional HREC and head of the institution:

This research has been approved by the National Health Research Ethics Committee (NHREC) of Nigeria and the Health Studies Research and Ethics Committee (HSREC), Faculty of Human Sciences, University of South Africa. If you have any questions regarding your rights as a research participant or have concern that your rights have been violated in the course of your participation in this study, please contact the NHREC using the following: 
National Health Research Ethics Committee (NHREC)

Department of Health Planning, Research \& Statistics

Federal Ministry of Health, Abuja

e-mail: chairman@nhrec.net, deskofficer@nhrec.net

In addition, if you have any question about your participation in this research, you can contact the principal investigator, (Robert Joseph Chiegil, Plot 1073-A1, JS Tarka Street, Area 3, Garki, Abuja, e-mail: rjchiegil@yahoo.com, phone number: 08035615573.

\section{PLEASE KEEP A COPY OF THE SIGNED INFORMED CONSENT.}

ATTENTION RESEARCHER: Note that you should include the Ethics Committee assigned approval number and the date/duration of the ethics committee approval on each copy of the consent form that is given to patients. 


\section{Annexure $F$}

Focus group discussion guide 


\section{Discussion Guide}

Title:

Nigerian health service users' views concerning

quality of antiretroviral therapy in public health

facilities

1. Welcome participants

a. Conduct self-introduction

b. Seek consent to participate and obtain signed consent from each participant.

c. Urge participants to ensure confidentiality of what each one says during the discussion: Since we are discussing as a group, I will not be able to guarantee confidentiality in what you say in this group. Therefore, if you would feel uncomfortable with any of your statements being shared with others in or outside this group, please, do not share them during the process

d. Record their bio-data: [probe to obtain individual information such as the following;

i. age,

ii. sex,

iii. occupation and

iv. highest educational attainment

2. For how long have you been attending ART care in this hospital [probe to obtain feedback from each one of the participants]?

3. What are your views regarding quality of ART care received in this hospital? [probe to obtain details on the following:]

a. Hospital leadership style (encourage employees to provide quality care, relationship with stakeholders)

b. Resources: Cleanliness of the ART service environment, availability and quality of drugs and health commodities, equipment, hospital furniture. 
c. Service of doctor (quick response to request for service, intention and attention of doctors in listening to a patient, diagnosing method, treatment explanation, courtesy).

d. Service of nurses (quick response to request for nursing service, help and attention of nurses, courtesy).

e. Service of other ART service officers, i.e. lab, pharmacy, medical records, staff (quick response to request for ART services, courtesy of lab staff, courtesy of pharmacy staff, courtesy of medical records staff, availability and reception of information (case files, next appointment date, referrals, etc), availability of drugs, tests and test results.

f. Other services (adherence counseling, counseling and testing, support group activities, PMTCT services, TB/HIV care, measures to control infection control in hospital, etc).

g. Hospital policy and strategy (strategies for ensuring that patients emerge from the hospital healthy)

h. Partnership and relationship of the hospital with the target communities, NGOs/CBOs, support group, etc.

i. Feedback from staff, community/society and support groups are used to improve services

4. What are your requirements and expectations regarding quality of ART care when you visit this hospital? [probe for the following expectations]

a. Hospital leadership style (encourage employees to provide quality care, relationship with stakeholders)

b. Quality of staff (attitude, availability, experience)

c. Resources (availability and quality of health commodities, equipment, infrastructure)

d. Hospital policy and strategy (patient focused, patient care goals and strategies are prioritized?)

e. Partnership and relationship of the hospital with the target communities. 
f. What is the hospital's current capacity to manage patient complaints regarding quality of care, compared to others in the region

5. What are the quality gaps you have noticed between your expectations and available services in the hospital?

6. What are your recommendations for improving quality of care and closing the service gaps? 


\section{DATA COLLECTION PROTOCOL IN HOSPITAL ' $X$ '}

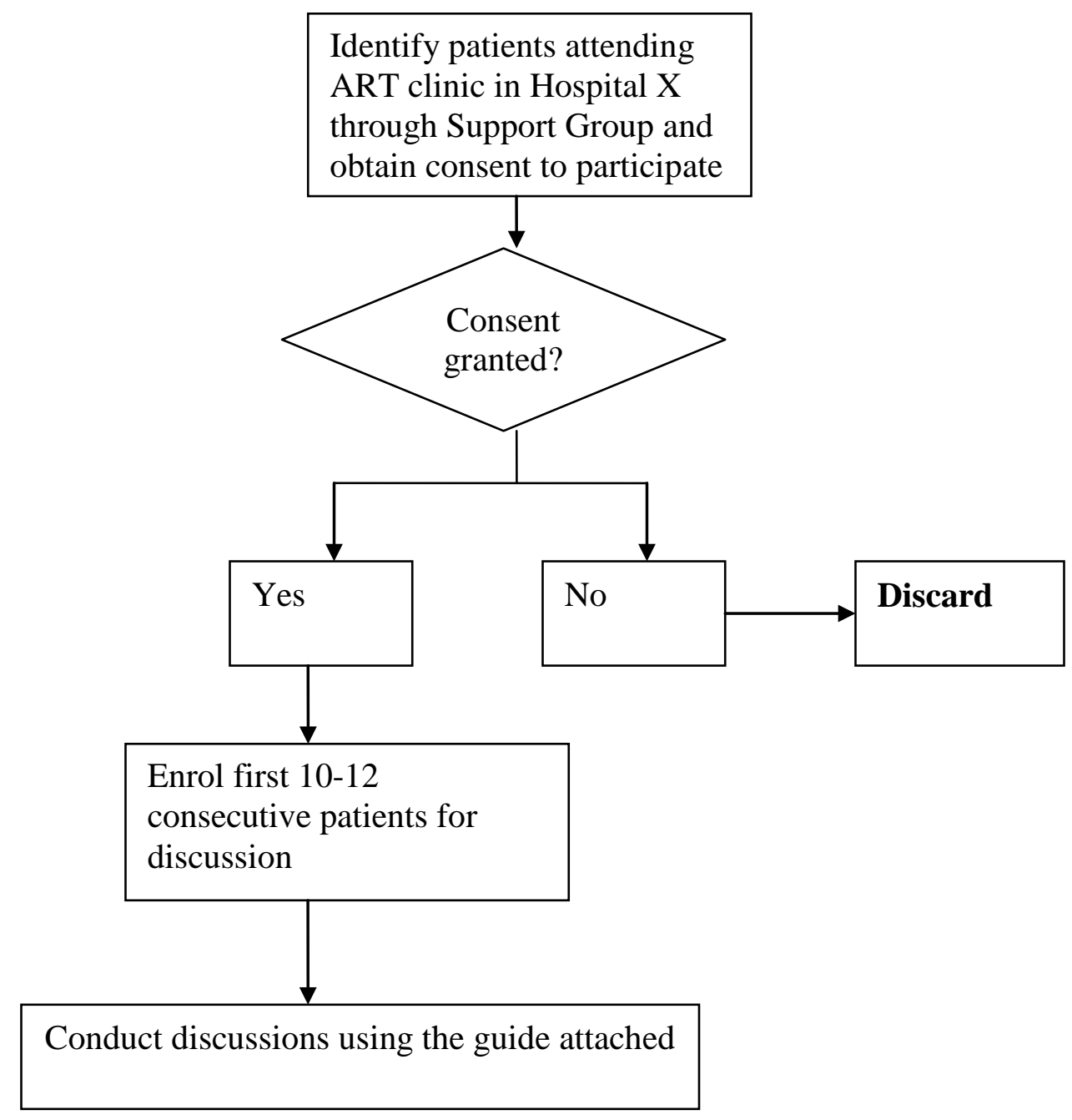




\section{Annexure G}

Maps showing the 6 geopolitical zones of Nigeria 
Map of Nigeria showing state geopolitical zones and population density by Land Scan [36].

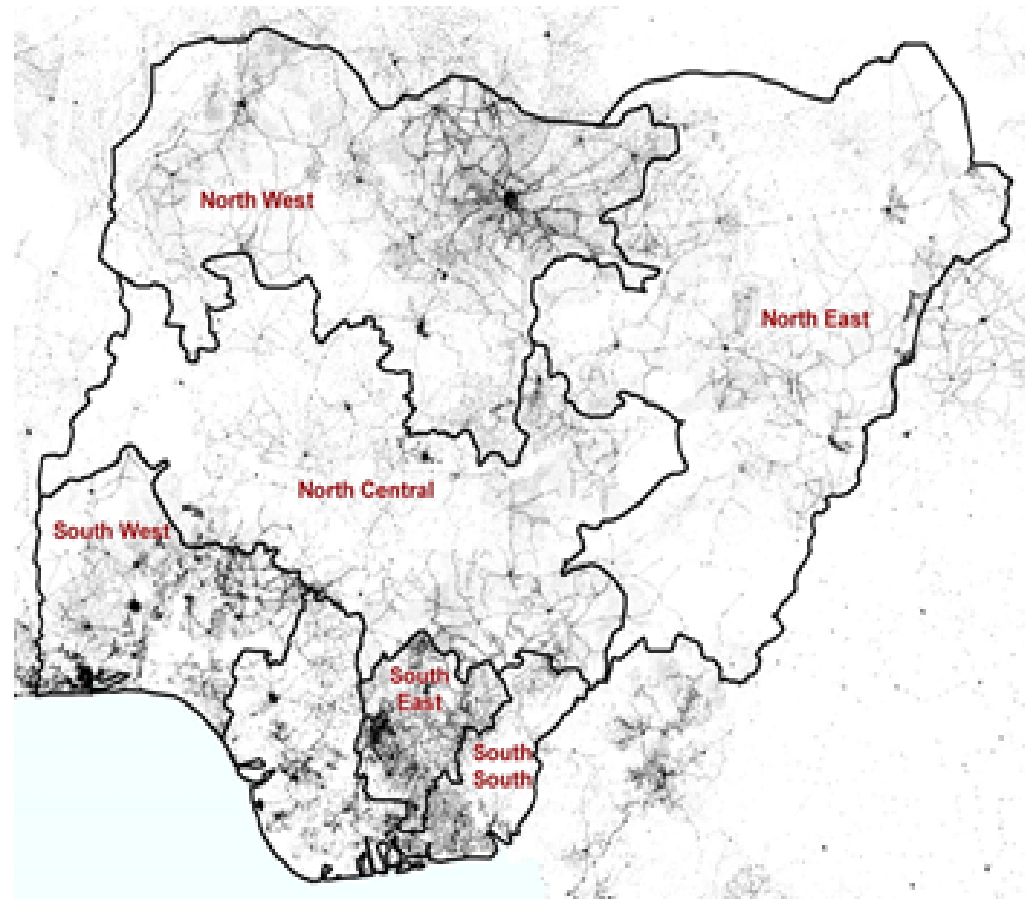

Wassllak $\mathrm{S}$ et al. J Infect Dis. 2011;203:098-s09 


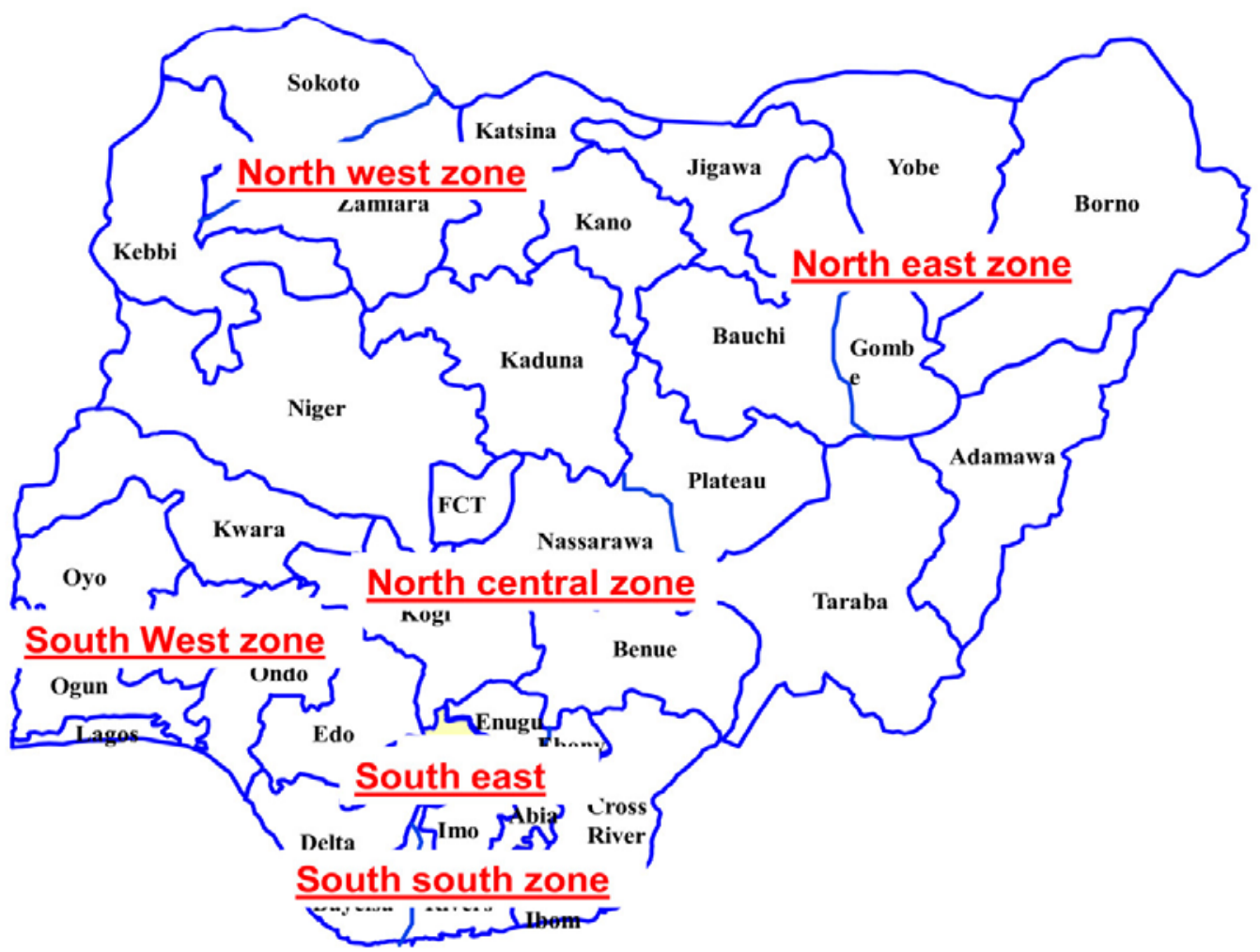

The Map of $\mathbf{3 6}$ states of Nigeria and the $\mathbf{6}$ geopolitical zones

Source: Dineen et al. BMC Ophthalmology 2008 8:17 